\title{
Palladium-Catalyzed C-H Allylation of Electron-Deficient Polyfluoroarenes with gem-Difluorinated Cyclopropanes
}

Ping-Xin Zhou, ${ }^{* a}$ Xiaozhe Yang, ${ }^{a}$ Jia Wang, ${ }^{a}$ Chunpo Ge, ${ }^{a}$ Wang Feng, ${ }^{a}$ Yong-Min Liang ${ }^{* b}$ and Yu Zhang $*^{\mathrm{a}}$

${ }^{a}$ School of Basic Medical Sciences, Xinxiang Medical University, Xinxiang, China

E-mail: zhoupingxin518@y163.com,wangjia1990@xxmu.edu.cn and zhangyuxxmu@126.com

Table of Contents

1 General remarks 2

2 Spectral data of $\mathbf{1 h}, \mathbf{1 p}, \mathbf{1 r}, \mathbf{2 e}$ and $\mathbf{2 f} \quad 2$

$3 \quad$ General procedure for the preparation of the products $\mathbf{3}$ and $\mathbf{4} 3$

4 Procedure for gram scale reaction 4

$5 \quad$ Spectral data of compound $\mathbf{3}$ and $\mathbf{4} \quad 4$

6 References 15

$7 \quad$ The crystal structure of product 3e 15

$8 \quad{ }^{1} \mathrm{H},{ }^{13} \mathrm{C}$ and ${ }^{19} \mathrm{~F}$ NMR spectra for compound $\mathbf{1 h}, \mathbf{1 p}, \mathbf{1 r}, \mathbf{2 e}, \mathbf{2 f}, \mathbf{3}$ and $\mathbf{4} \quad 18$ 


\section{General remarks}

The desired product was purified by flash column chromatography, silica gel (200 300 mesh). ${ }^{1} \mathrm{H}$ NMR spectra and ${ }^{13} \mathrm{C}$ NMR spectra were recorded on $400 \mathrm{MHz}$ in $\mathrm{CDCl}_{3}$ and TMS as internal standard. All products were further characterized by HRMS (high resolution mass spectra). Copies of their ${ }^{1} \mathrm{H}$ NMR and ${ }^{13} \mathrm{C}$ NMR spectra are provided. All solvents were dried and distilled according to standard procedures. Commercially available reagents and solvents were used without further purification. Compounds $\mathbf{1}$ were synthesized according to the literature procedure ${ }^{1}$ and NMR data have matched to literatures ${ }^{1,2}$. For gem-difluorinated cyclopropanes $\mathbf{1 h}, \mathbf{1 p}$ and $\mathbf{1 r}$ are new compounds. For fluoroarenes $\mathbf{2 e}$ was prepared from 2,3,5,6-tetrafluorobenzyl alcohol and tert-Butyldimethylsilyl chloride based on reported procedures. ${ }^{3}$ For fluoroarenes $2 \mathbf{f}$ was prepared from 2,3,5,6-tetrafluorobenzyl alcohol and benzyl bromide based on reported procedures. ${ }^{4}$ All reactions were heated by oil bath. HRMS analysis of compounds was performed with a time-of-flight mass spectrometer (micrOTOF-Q, Bruker Daltonik, Germany).

\section{Spectral data of $1 \mathrm{~h}, 1 \mathrm{p}, 1 \mathrm{r}, 2 \mathrm{e}$ and $2 \mathrm{f}$}

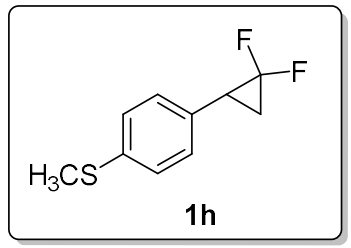

(4-(2,2-difluorocyclopropyl)phenyl)(methyl)sulfane: $\mathrm{Rf}=0.30$ (peterolium ether/ethyl acetate, 100:1); Column chromatoghraphy(silica gel; peterolium ether/ethyl acetate, 200:1); colourless oil; ${ }^{1} \mathrm{H} \operatorname{NMR}\left(400 \mathrm{MHz}, \mathrm{CDCl}_{3}\right) \delta: 7.21(\mathrm{~d}, J=8.4 \mathrm{~Hz}, 2 \mathrm{H}), 7.13(\mathrm{~d}, J=8.4 \mathrm{~Hz}, 2 \mathrm{H}), 2.75-2.66(\mathrm{~m}, 1 \mathrm{H})$, $2.47(\mathrm{~s}, 3 \mathrm{H}), 1.85-1.75(\mathrm{~m}, 1 \mathrm{H}), 1.62-1.54(\mathrm{~m}, 1 \mathrm{H}) ;{ }^{13} \mathrm{C} \mathrm{NMR}\left(100 \mathrm{MHz}, \mathrm{CDCl}_{3}\right)$ 8: 137.3, 130.4, $128.5,126.7,112.5(\mathrm{dd}, J=282.2,285.4 \mathrm{~Hz}), 26.7(\mathrm{t}, J=11.4 \mathrm{~Hz}), 16.9(\mathrm{t}, J=10.5 \mathrm{~Hz}), 15.8 ;{ }^{19} \mathrm{~F}$ NMR $\left(376 \mathrm{MHz}, \mathrm{CDCl}_{3}\right) \delta:-125.9(\mathrm{~d}, J=153.4 \mathrm{~Hz}, 1 \mathrm{~F}),-142.2(\mathrm{~d}, J=153.4 \mathrm{~Hz}, 1 \mathrm{~F}) ; \operatorname{HRMS}(\mathrm{ESI}) \mathrm{m} / \mathrm{z}$ : $[\mathrm{M}+\mathrm{H}]^{+}$calcd for $\mathrm{C}_{10} \mathrm{H}_{11} \mathrm{~F}_{2} \mathrm{~S} 201.0544$; found 201.0539.

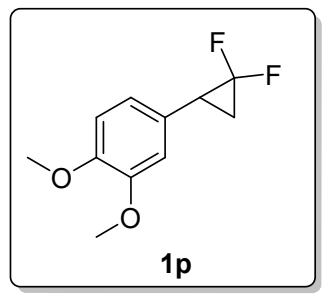

4-(2,2-difluorocyclopropyl)-1,2-dimethoxybenzene; $\mathrm{Rf}=0.28$ (peterolium ether/ethyl acetate, 30:1); Column chromatoghraphy(silica gel; peterolium ether/ethyl acetate, 60:1); colourless oil; ${ }^{1} \mathrm{H}$ $\operatorname{NMR}\left(400 \mathrm{MHz}, \mathrm{CDCl}_{3}\right) \delta: 6.84-6.75(\mathrm{~m}, 3 \mathrm{H}), 3.88(\mathrm{~s}, 3 \mathrm{H}), 3.87(\mathrm{~s}, 3 \mathrm{H}), 2.76-2.67(\mathrm{~m}, 1 \mathrm{H})$, $1.80-1.74(\mathrm{~m}, 1 \mathrm{H}), 1.61-1.53(\mathrm{~m}, 1 \mathrm{H}) ;{ }^{13} \mathrm{C} \mathrm{NMR}\left(100 \mathrm{MHz}, \mathrm{CDCl}_{3}\right) \delta: 148.8,148.2,125.9,120.2$, $112.6(\mathrm{dd}, J=282.1,284.8 \mathrm{~Hz}), 111.5,111.0,55.9,55.8,26.7(\mathrm{t}, J=11.4 \mathrm{~Hz}), 16.9(\mathrm{t}, J=10.4 \mathrm{~Hz}) ;{ }^{19} \mathrm{~F}$ NMR (376MHz, CDCl $) \delta:-126.0(\mathrm{~d}, J=153.0 \mathrm{~Hz}, 1 \mathrm{~F}),-142.0(\mathrm{~d}, J=153.0 \mathrm{~Hz}, 1 \mathrm{~F}) ; \mathrm{HRMS}(\mathrm{ESI}) \mathrm{m} / \mathrm{z}$ : $[\mathrm{M}+\mathrm{Na}]^{+}$calcd for $\mathrm{C}_{11} \mathrm{H}_{12} \mathrm{~F}_{2} \mathrm{O}_{2} \mathrm{Na} 237.0698$; found 237.0701. 


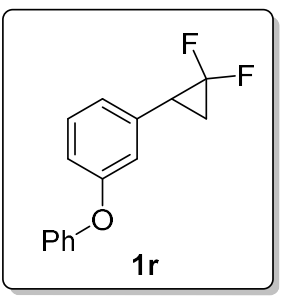

1-(2,2-difluorocyclopropyl)-3-phenoxybenzene: $\mathrm{Rf}=0.29$ (peterolium ether/ethyl acetate, 100:1); Column chromatoghraphy(silica gel; peterolium ether/ethyl acetate, 200:1); colourless oil; ${ }^{1} \mathrm{H}$ $\operatorname{NMR}\left(400 \mathrm{MHz}, \mathrm{CDCl}_{3}\right) \delta: 7.58-7.54(\mathrm{~m}, 4 \mathrm{H}), 7.45-7.41(\mathrm{~m}, 2 \mathrm{H}), 7.36-7.28(\mathrm{~m}, 3 \mathrm{H}), 2.82-2.74(\mathrm{~m}$, $1 \mathrm{H}), 1.89-1.79(\mathrm{~m}, 1 \mathrm{H}), 1.69-1.61(\mathrm{~m}, 1 \mathrm{H}) ;{ }^{13} \mathrm{C} \mathrm{NMR}\left(100 \mathrm{MHz}, \mathrm{CDCl}_{3}\right) \delta: 140.6,140.1,132.7$, $128.8,128.4,127.3,127.2,127.0,126.6,113.9,112.6(\mathrm{dd}, J=282.3,285.3 \mathrm{~Hz}), 26.9(\mathrm{t}, J=11.5 \mathrm{~Hz})$, $17.1(\mathrm{t}, J=10.4 \mathrm{~Hz}) ;{ }^{19} \mathrm{~F}$ NMR $\left(376 \mathrm{MHz}, \mathrm{CDCl}_{3}\right) \delta:-125.7(\mathrm{~d}, J=153.4 \mathrm{~Hz}, 1 \mathrm{~F}),-142.2(\mathrm{~d}, J=153.4 \mathrm{~Hz}$, 1F); HRMS(ESI) m/z: [M+H] $]^{+}$calcd for $\mathrm{C}_{15} \mathrm{H}_{13} \mathrm{~F}_{2} \mathrm{O} 247.0929$; found 247.0927 .

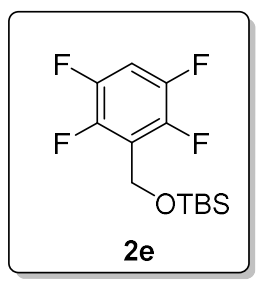

tert-butyldimethyl((2,3,5,6-tetrafluorobenzyl)oxy)silane: $\quad \mathrm{Rf}=0.32$ (peterolium ether/ethyl acetate, 100:1); Column chromatoghraphy(silica gel; peterolium ether/ethyl acetate, 200:1); colourless oil; ${ }^{1} \mathrm{H} \mathrm{NMR}\left(400 \mathrm{MHz}, \mathrm{CDCl}_{3}\right) \delta: 6.94-6.86(\mathrm{~m}, 1 \mathrm{H}), 4.68(\mathrm{t}, J=1.6 \mathrm{~Hz}, 2 \mathrm{H}), 0.79(\mathrm{~s}, 9 \mathrm{H})$, $0.007(\mathrm{~s}, 6 \mathrm{H}) ;{ }^{13} \mathrm{C} \mathrm{NMR}\left(100 \mathrm{MHz}, \mathrm{CDCl}_{3}\right) \delta: 145.9(\mathrm{dm}, J=246.3 \mathrm{~Hz}), 145.0(\mathrm{dm}, J=246.4 \mathrm{~Hz})$, $119.7(\mathrm{t}, J=17.3 \mathrm{~Hz}), 105.4(\mathrm{t}, J=22.4 \mathrm{~Hz}), 53.3,25.7,18.3,-5.6 ;{ }^{19} \mathrm{~F} \mathrm{NMR}\left(376 \mathrm{MHz}, \mathrm{CDCl}_{3}\right) \delta$ : $-139.5(\mathrm{~m}, 2 \mathrm{~F}),-144.4(\mathrm{~m}, 2 \mathrm{~F})$; HRMS(ESI) $\mathrm{m} / \mathrm{z}$ : $[\mathrm{M}+\mathrm{H}]^{+}$calcd for $\mathrm{C}_{13} \mathrm{H}_{19} \mathrm{~F}_{4} \mathrm{OSi}$ 295.1136; found 295.1139 .

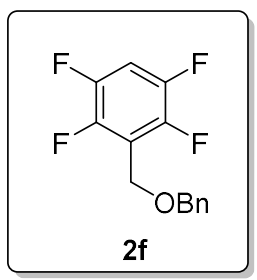

3-((benzyloxy)methyl)-1,2,4,5-tetrafluorobenzene: $\mathrm{Rf}=0.32$ (peterolium ether/ethyl acetate, 100:1); Column chromatoghraphy(silica gel; peterolium ether/ethyl acetate, 200:1); colourless oil; ${ }^{1} \mathrm{H}$ NMR(400MHz, $\left.\mathrm{CDCl}_{3}\right)$ 8: 7.36-7.33(m, 4H), 7.31-7.27(m, 1H), 7.08-6.99(m, 1H), 4.66(s, 2H), $4.59(\mathrm{~s}, 2 \mathrm{H}) ;{ }^{13} \mathrm{C} \mathrm{NMR}\left(100 \mathrm{MHz}, \mathrm{CDCl}_{3}\right) \delta: 145.8(\mathrm{dm}, J=246.8 \mathrm{~Hz}), 145.1(\mathrm{dm}, J=247.1 \mathrm{~Hz}), 137.3$, $128.5,127.9,127.8,117.0(\mathrm{t}, J=17.6 \mathrm{~Hz}), 106.1(\mathrm{t}, J=22.4 \mathrm{~Hz}), 72.9,59.3(\mathrm{t}, J=17.6 \mathrm{~Hz}) ;{ }^{19} \mathrm{~F}$ NMR $\left(376 \mathrm{MHz}, \mathrm{CDCl}_{3}\right) \delta:-139.1(\mathrm{~m}, 2 \mathrm{~F}),-143.3(\mathrm{~m}, 2 \mathrm{~F}) ; \mathrm{HRMS}(\mathrm{ESI}) \mathrm{m} / \mathrm{z}:[\mathrm{M}+\mathrm{Na}]^{+}$calcd for $\mathrm{C}_{14} \mathrm{H}_{10} \mathrm{~F}_{4} \mathrm{ONa} 293.0560$; found 293.0561.

\section{General procedure for the preparation of the products 3 and 4}

An oven-dried Schlenk tube under a nitrogen atmosphere was charged with gem-difluorinated cyclopropanes 1 ( $0.3 \mathrm{mmol}, 1.0$ equiv), polyfluoroarenes 2 (0.6 mmol, 2.0 equiv), $\mathrm{Pd}(\mathrm{OTFA})_{2}(10 \mathrm{~mol} \%), \mathrm{P}^{\mathrm{t}} \mathrm{Bu}_{3} \cdot \mathrm{HBF}_{4}(20 \mathrm{~mol} \%), \mathrm{K}_{3} \mathrm{PO}_{4}(0.60 \mathrm{mmol}, 2.0$ equiv.), dioxane 
$(1.5 \mathrm{~mL})$. The mixture was stirred at room temperature for 20 minutes and then stirred at $90{ }^{\circ} \mathrm{C}$ for $16 \mathrm{~h}$. The resulting mixture was cooled to room temperature and filtered through Celite eluting with EtOAc. The volatiles were evaporated under reduced pressure and the residue was purified by silica gel flash chromatography to afford the desired products 3 .

\section{Procedure for gram scale reaction}

An oven-dried Schlenk tube under a nitrogen atmosphere was charged with gem-difluorinated cyclopropanes 1 (5.0 mmol, 1.02g, 1.0 equiv), polyfluoroarenes 2 (10.0 mmol, $1.8 \mathrm{~g}, 2.0$ equiv), $\mathrm{Pd}(\mathrm{OTFA})_{2}\left(0.5 \mathrm{mmol}, 0.166 \mathrm{~g}, 10 \mathrm{~mol} \%\right.$, ), $\mathrm{P}^{\mathrm{t}} \mathrm{Bu}_{3} \cdot \mathrm{HBF}_{4}(1.0 \mathrm{mmol}, 0.29 \mathrm{~g}, 20$ $\mathrm{mol} \%), \mathrm{K}_{3} \mathrm{PO}_{4}(10.0 \mathrm{mmol}, 2.12 \mathrm{~g}, 2.0$ equiv.), dioxane $(20 \mathrm{~mL})$. The mixture was stirred at room temperature for 20 minutes and then stirred at $90{ }^{\circ} \mathrm{C}$ for $16 \mathrm{~h}$. The resulting mixture was cooled to room temperature and filtered through Celite eluting with EtOAc. The volatiles were evaporated under reduced pressure and the residue was purified by silica gel flash chromatography (peterolium ether/ethyl acetate, 200:1) to afford the desired products $\mathbf{3 a}(4.35 \mathrm{mmol}, 1.58 \mathrm{~g}, 87 \%$ ).

\section{Spectral data of compound 3 and 4}

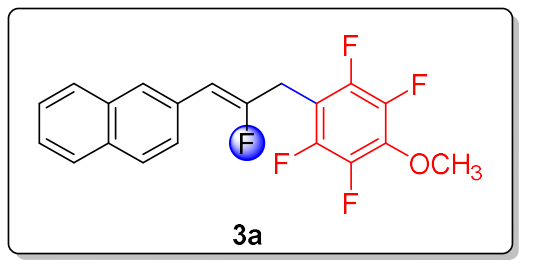

(Z)-2-(2-fluoro-3-(2,3,5,6-tetrafluoro-4-methoxyphenyl)prop-1-en-1-yl)naphthalene:

$\mathrm{Rf}=0.32$ (peterolium ether/ethyl acetate, 100:1); Column chromatoghraphy(silica gel; peterolium ether/ethyl acetate, 200:1); 98.2mg, 90\%; white solid; ${ }^{1} \mathrm{H} \mathrm{NMR}\left(400 \mathrm{MHz}, \mathrm{CDCl}_{3}\right) \delta: 7.85(\mathrm{~s}, 1 \mathrm{H})$, 7.77-7.74 (m, 3H), 7.60(dd, $J=1.6,8.8 \mathrm{~Hz}, 1 \mathrm{H}), 7.44-7.41(\mathrm{~m}, 2 \mathrm{H}), 5.70(\mathrm{~d}, J=38.0 \mathrm{~Hz}, 1 \mathrm{H}), 4.05(\mathrm{~s}$, $3 \mathrm{H}), 3.72(\mathrm{~d}, J=15.6 \mathrm{~Hz}, 2 \mathrm{H}) ;{ }^{13} \mathrm{C} \mathrm{NMR}\left(100 \mathrm{MHz}, \mathrm{CDCl}_{3}\right) \delta: 157.3,154.6,145.5(\mathrm{dm}, J=245.0 \mathrm{~Hz})$, $140.9(\mathrm{dm}, J=245.9 \mathrm{~Hz}), 133.3,132.4(\mathrm{~d}, J=1.6 \mathrm{~Hz}), 130.4(\mathrm{~d}, J=3.1 \mathrm{~Hz}), 127.9,127.9,127.5,127.5$, $126.3,126.2,126.1,125.9,107.5(\mathrm{~d}, J=7.4 \mathrm{~Hz}), 62.0(\mathrm{t}, J=3.6 \mathrm{~Hz}), 26.2(\mathrm{~d}, J=30.6 \mathrm{~Hz}) ;{ }^{19} \mathrm{~F}$ NMR $\left(376 \mathrm{MHz}, \mathrm{CDCl}_{3}\right) \delta:-102.2(\mathrm{~m}, 1 \mathrm{~F}),-144.5(\mathrm{~m}, 2 \mathrm{~F}),-157.8(\mathrm{~m}, 2 \mathrm{~F}) ; \mathrm{HRMS}(\mathrm{APCI}) \mathrm{m} / \mathrm{z}:[\mathrm{M}+\mathrm{H}]^{+}$ calcd for $\mathrm{C}_{20} \mathrm{H}_{14} \mathrm{~F}_{5} \mathrm{O} 365.0959$; found 365.0956 .

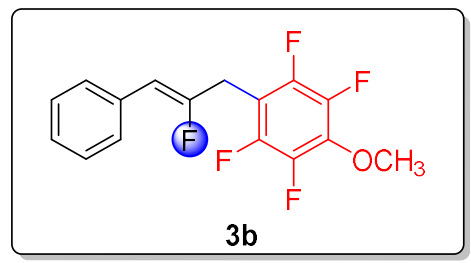

(Z)-1,2,4,5-tetrafluoro-3-(2-fluoro-3-phenylallyl)-6-methoxybenzene; $\quad \mathrm{Rf}=0.31$ (peterolium ether/ethyl acetate, 100:1); Column chromatoghraphy(silica gel; peterolium ether/ethyl acetate, 200:1); 77.2mg, 82\%; colourless oil; ${ }^{1} \mathrm{H} \operatorname{NMR}\left(400 \mathrm{MHz}, \mathrm{CDCl}_{3}\right) \delta: 7.44(\mathrm{~d}, J=7.6 \mathrm{~Hz}, 2 \mathrm{H})$, $7.32-7.28(\mathrm{~m}, 2 \mathrm{H}), 7.24-7.20(\mathrm{~m}, 1 \mathrm{H}), 5.56(\mathrm{~d}, J=38.0 \mathrm{~Hz}, 1 \mathrm{H}), 4.07$ (s, $3 \mathrm{H}), 3.70(\mathrm{~d}, J=15.6 \mathrm{~Hz}, 2 \mathrm{H})$; ${ }^{13} \mathrm{C}$ NMR(100MHz, $\left.\mathrm{CDCl}_{3}\right) \delta: 157.0,154.3,145.6(\mathrm{dm}, J=244.8 \mathrm{~Hz}), 141.0(\mathrm{dm}, J=245.8 \mathrm{~Hz})$, $132.8(\mathrm{~d}, J=2.8 \mathrm{~Hz}), 128.4(\mathrm{~m}), 127.2(\mathrm{~d}, J=2.3 \mathrm{~Hz}), 115.3(\mathrm{~d}, J=21.3 \mathrm{~Hz}), 107.8(\mathrm{~m}), 107.4(\mathrm{~d}$, $J=7.6 \mathrm{~Hz}), 62.1(\mathrm{t}, J=3.6 \mathrm{~Hz}), 26.2(\mathrm{~d}, J=30.6 \mathrm{~Hz}) ;{ }^{19} \mathrm{~F} \mathrm{NMR}\left(376 \mathrm{MHz}, \mathrm{CDCl}_{3}\right) \delta:-102.7(\mathrm{~m}, 1 \mathrm{~F})$, $-144.6(\mathrm{~m}, 2 \mathrm{~F}),-157.9(\mathrm{~m}, 2 \mathrm{~F})$; HRMS(APCI) $\mathrm{m} / \mathrm{z}$ : $[\mathrm{M}]^{+}$calcd for $\mathrm{C}_{16} \mathrm{H}_{11} \mathrm{~F}_{5} \mathrm{O}$ 314.0730; found 314.0723 . 


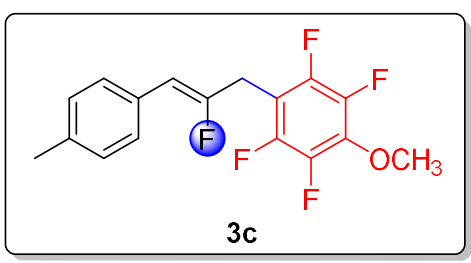

(Z)-1,2,4,5-tetrafluoro-3-(2-fluoro-3-(p-tolyl)allyl)-6-methoxybenzene; $\quad \mathrm{Rf}=0.33$ (peterolium ether/ethyl acetate, 100:1); Column chromatoghraphy(silica gel; peterolium ether/ethyl acetate, 200:1); 62.1mg, 63\%; white solid; ${ }^{1} \mathrm{H}$ NMR (400MHz, $\left.\mathrm{CDCl}_{3}\right) \delta: 7.33(\mathrm{~d}, J=8.0 \mathrm{~Hz}, 2 \mathrm{H}), 7.11(\mathrm{~d}$, $J=8.4 \mathrm{~Hz}, 2 \mathrm{H}), 5.52(\mathrm{~d}, J=38.4 \mathrm{~Hz}, 1 \mathrm{H}), 4.06(\mathrm{~s}, 3 \mathrm{H}), 3.68(\mathrm{~d}, J=15.6 \mathrm{~Hz}, 2 \mathrm{H}), 2.31(\mathrm{~s}, 3 \mathrm{H}) ;{ }^{13} \mathrm{C}$ NMR $\left(100 \mathrm{MHz}, \mathrm{CDCl}_{3}\right) \delta: 156.4,153.7,145.5(\mathrm{dm}, J=244.9 \mathrm{~Hz}), 141.2(\mathrm{dm}, J=245.8 \mathrm{~Hz}), 137.0(\mathrm{~d}$, $J=2.3 \mathrm{~Hz}), 130.0(\mathrm{~d}, J=2.8 \mathrm{~Hz}), 129.1,128.3(\mathrm{~d}, J=7.2 \mathrm{~Hz}), 107.9(\mathrm{~m}), 107.3(\mathrm{~d}, J=7.8 \mathrm{~Hz}), 62.1(\mathrm{t}$, $J=3.6 \mathrm{~Hz}), 26.1(\mathrm{~d}, J=30.7 \mathrm{~Hz}), 21.1 ;{ }^{19} \mathrm{~F} \mathrm{NMR}\left(376 \mathrm{MHz}, \mathrm{CDCl}_{3}\right) \delta:-103.8(\mathrm{~m}, 1 \mathrm{~F}),-144.6(\mathrm{dd}$, $J=5.3,12.8 \mathrm{~Hz}, 2 \mathrm{~F}),-158.0(\mathrm{~m}, 2 \mathrm{~F})$; HRMS(APCI) $\mathrm{m} / \mathrm{z}:[\mathrm{M}+\mathrm{H}]^{+}$calcd for $\mathrm{C}_{17} \mathrm{H}_{14} \mathrm{~F}_{5} \mathrm{O} 329.0959$; found 329.0961 .

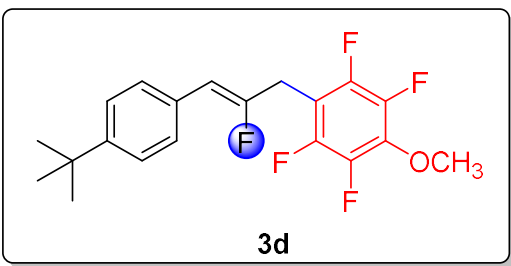

\section{(Z)-1-(3-(4-(tert-butyl)phenyl)-2-fluoroallyl)-2,3,5,6-tetrafluoro-4-methoxybenzene;}

$\mathrm{Rf}=0.32$ (peterolium ether/ethyl acetate, 100:1); Column chromatoghraphy(silica gel; peterolium ether/ethyl acetate, 200:1); 91.0mg, 82\%; colourless oil; ${ }^{1} \mathrm{H}$ NMR $\left(400 \mathrm{MHz}, \mathrm{CDCl}_{3}\right) \delta: 7.35(\mathrm{dd}$, $J=8.4,22.8 \mathrm{~Hz}, 4 \mathrm{H}), 5.55(\mathrm{~d}, J=38.8 \mathrm{~Hz}, 1 \mathrm{H}), 4.07(\mathrm{~s}, 3 \mathrm{H}), 3.69(\mathrm{~d}, J=16.0 \mathrm{~Hz}, 2 \mathrm{H}), 1.3(\mathrm{~s}, 9 \mathrm{H}) ;{ }^{13} \mathrm{C}$ NMR $\left(100 \mathrm{MHz}, \mathrm{CDCl}_{3}\right) \delta: 156.5,153.9,150.3(\mathrm{~d}, J=2.1 \mathrm{~Hz}), 145.5(\mathrm{dm}, J=244.9 \mathrm{~Hz}), 140.9(\mathrm{dm}$, $J=246.4 \mathrm{~Hz}), 130.0(\mathrm{~d}, J=2.8 \mathrm{~Hz}), 128.1(\mathrm{~d}, J=7.2 \mathrm{~Hz}), 125.3,108.0(\mathrm{~m}), 107.2(\mathrm{~d}, J=7.9 \mathrm{~Hz}), 62.1(\mathrm{t}$, $J=3.6 \mathrm{~Hz}), 34.5,31.2,26.2(\mathrm{~d}, J=30.5 \mathrm{~Hz}) ;{ }^{19} \mathrm{~F}$ NMR $\left(376 \mathrm{MHz}, \mathrm{CDCl}_{3}\right) \delta:-103.7(\mathrm{~m}, 1 \mathrm{~F}),-144.6(\mathrm{~m}$, $2 \mathrm{~F}),-158.0(\mathrm{~m}, 2 \mathrm{~F})$; HRMS(APCI) $\mathrm{m} / \mathrm{z}$ : [M] $]^{+}$calcd for $\mathrm{C}_{20} \mathrm{H}_{19} \mathrm{~F}_{5} \mathrm{O} 370.1356$; found 370.1352 .

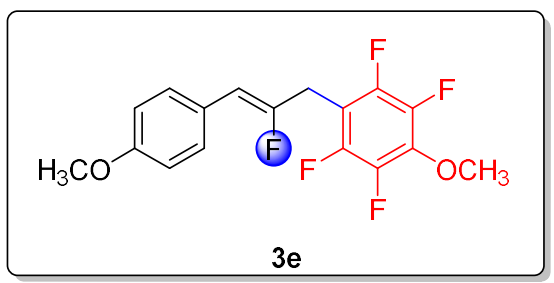

(Z)-1,2,4,5-tetrafluoro-3-(2-fluoro-3-(4-methoxyphenyl)allyl)-6-methoxybenzene;

$\mathrm{Rf}=0.26$ (peterolium ether/ethyl acetate, 100:1); Column chromatoghraphy(silica gel; peterolium ether/ethyl acetate, 200:1); 66.1mg, 64\%; yellow solid; ${ }^{1} \mathrm{H}$ NMR $\left(400 \mathrm{MHz}, \mathrm{CDCl}_{3}\right) \delta: 7.38(\mathrm{~d}$, $J=8.8 \mathrm{~Hz}, 2 \mathrm{H}), 6.83(\mathrm{~d}, J=8.8 \mathrm{~Hz}, 2 \mathrm{H}), 5.50(\mathrm{~d}, J=38.4 \mathrm{~Hz}, 1 \mathrm{H}), 4.06(\mathrm{~s}, 3 \mathrm{H}), 3.79(\mathrm{~s}, 3 \mathrm{H}), 3.67(\mathrm{~d}$, $J=15.6 \mathrm{~Hz}, 2 \mathrm{H}) ;{ }^{13} \mathrm{C} \mathrm{NMR}\left(100 \mathrm{MHz}, \mathrm{CDCl}_{3}\right) \delta: 158.6(\mathrm{~d}, J=2.8 \mathrm{~Hz}), 155.6,153.0,145.5(\mathrm{dm}$, $J=244.8 \mathrm{~Hz}), 140.9(\mathrm{dm}, J=241.5 \mathrm{~Hz}), 129.6(\mathrm{~d}, J=7.4 \mathrm{~Hz}), 125.5(\mathrm{~d}, J=2.9 \mathrm{~Hz}), 113.8,108.0(\mathrm{t}$, $J=18.2 \mathrm{~Hz}), 106.8(\mathrm{~d}, J=8.0 \mathrm{~Hz}), 62.1(\mathrm{t}, J=3.5 \mathrm{~Hz}), 55.1,26.1(\mathrm{~d}, J=30.7 \mathrm{~Hz}) ;{ }^{19} \mathrm{~F}$ NMR $(376 \mathrm{MHz}$, $\left.\mathrm{CDCl}_{3}\right) \delta:-105.7(\mathrm{~m}, 1 \mathrm{~F}),-144.7(\mathrm{dd}, J=5.3,12.4 \mathrm{~Hz}, 2 \mathrm{~F}),-158.0(\mathrm{~m}, 2 \mathrm{~F}) ; \operatorname{HRMS}(\mathrm{APCI}) \mathrm{m} / \mathrm{z}$ : $[\mathrm{M}+\mathrm{H}]^{+}$calcd for $\mathrm{C}_{17} \mathrm{H}_{14} \mathrm{~F}_{5} \mathrm{O}_{2} 345.0908$; found 345.0904 . 


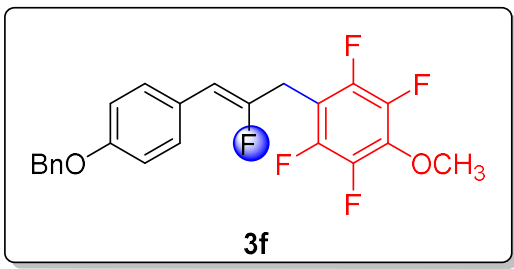

(Z)-1-(3-(4-(benzyloxy)phenyl)-2-fluoroallyl)-2,3,5,6-tetrafluoro-4-methoxybenzene;

$\mathrm{Rf}=0.27$ (peterolium ether/ethyl acetate, 100:1); Column chromatoghraphy(silica gel; peterolium ether/ethyl acetate, 200:1); 99.5mg, 79\%; white solid; ${ }^{1} \mathrm{H}$ NMR (400MHz, $\left.\mathrm{CDCl}_{3}\right)$ 8: 7.31-7.24(m, $6 \mathrm{H}), 7.22-7.20(\mathrm{~m}, 1 \mathrm{H}), 6.79(\mathrm{~d}, J=8.8 \mathrm{~Hz}, 2 \mathrm{H}), 5.39(\mathrm{~d}, J=38.4 \mathrm{~Hz}, 1 \mathrm{H}), 4.92(\mathrm{~s}, 2 \mathrm{H}), 3.94(\mathrm{~s}, 3 \mathrm{H})$, $3.55(\mathrm{~d}, J=16.0 \mathrm{~Hz}, 2 \mathrm{H}) ;{ }^{13} \mathrm{C} \mathrm{NMR}\left(100 \mathrm{MHz}, \mathrm{CDCl}_{3}\right) \delta: 157.8(\mathrm{~d}, J=2.8 \mathrm{~Hz}), 155.7,153.0,145.5(\mathrm{dm}$, $J=249.5 \mathrm{~Hz}), 140.9(\mathrm{dm}, J=245.7 \mathrm{~Hz}), 139.6(\mathrm{~m}), 136.8,129.6(\mathrm{~d}, J=7.4 \mathrm{~Hz}), 128.5,127.9,125.8(\mathrm{~d}$, $J=2.8 \mathrm{~Hz}), 114.7,108.0(\mathrm{~d}, J=18.4 \mathrm{~Hz}), 106.8(\mathrm{~d}, J=8.0 \mathrm{~Hz}), 69.8,62.0(\mathrm{t}, J=3.6 \mathrm{~Hz}), 26.0(\mathrm{~d}$, $J=30.6 \mathrm{~Hz}) ;{ }^{19} \mathrm{~F}$ NMR $\left(376 \mathrm{MHz}, \mathrm{CDCl}_{3}\right) \delta:-105.5(\mathrm{~m}, 1 \mathrm{~F}),-144.6(\mathrm{dd}, J=5.3,12.8 \mathrm{~Hz}, 2 \mathrm{~F})$, $-158.0(\mathrm{~m}, 2 \mathrm{~F})$; $\mathrm{HRMS}(\mathrm{ESI}) \mathrm{m} / \mathrm{z}$ : $\left[\mathrm{M}+\mathrm{NH}_{4}\right]^{+}$calcd for $\mathrm{C}_{23} \mathrm{H}_{21} \mathrm{~F}_{5} \mathrm{NO}_{2} 438.1487$; found 438.1485 .

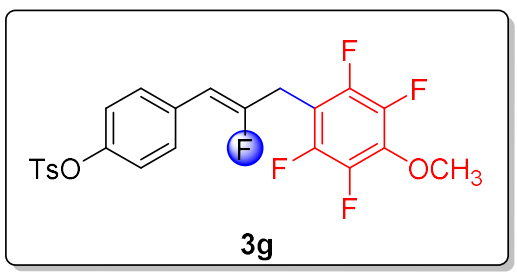

\section{(Z)-4-(2-fluoro-3-(2,3,5,6-tetrafluoro-4-methoxyphenyl)prop-1-en-1-yl)phenyl}

4-methylbenzenesulfonate; $\quad \mathrm{Rf}=0.26$ (peterolium ether/ethyl acetate, 10:1); Column chromatoghraphy(silica gel; peterolium ether/ethyl acetate, $30: 1) ; 124.9 \mathrm{mg}, 86 \%$; yellow solid; ${ }^{1} \mathrm{H}$ NMR $\left(400 \mathrm{MHz}, \mathrm{CDCl}_{3}\right) \delta: 7.67(\mathrm{~d}, J=8.0 \mathrm{~Hz}, 2 \mathrm{H}), 7.36-7.28(\mathrm{~m}, 4 \mathrm{H}), 6.90(\mathrm{~d}, J=8.8 \mathrm{~Hz}, 2 \mathrm{H}), 5.51(\mathrm{~d}$, $J=37.6 \mathrm{~Hz}, 1 \mathrm{H}), 4.07(\mathrm{~s}, 3 \mathrm{H}), 3.68(\mathrm{~d}, J=15.6 \mathrm{~Hz}, 2 \mathrm{H}), 2.43(\mathrm{~s}, 3 \mathrm{H}) ;{ }^{13} \mathrm{C} \mathrm{NMR}\left(100 \mathrm{MHz}, \mathrm{CDCl}_{3}\right) \delta$ : $157.6,154.9,148.2(\mathrm{~d}, J=3.3 \mathrm{~Hz}), 145.5(\mathrm{dm}, J=240.9 \mathrm{~Hz}), 145.4,140.9(\mathrm{dm}, J=245.9 \mathrm{~Hz}), 132.1$, $131.8(\mathrm{~d}, J=2.8 \mathrm{~Hz}), 129.7,129.5(\mathrm{~d}, J=7.7 \mathrm{~Hz}), 128.4,122.3,107.3(\mathrm{~m}), 106.2(\mathrm{~d}, J=7.5 \mathrm{~Hz}), 62.0(\mathrm{t}$, $J=3.6 \mathrm{~Hz}), 26.0(\mathrm{~d}, J=30.4 \mathrm{~Hz}), 21.6 ;{ }^{19} \mathrm{~F} \mathrm{NMR}\left(376 \mathrm{MHz}, \mathrm{CDCl}_{3}\right) \delta:-101.9(\mathrm{~m}, 1 \mathrm{~F}),-144.6(\mathrm{dd}$, $J=4.9,12.7 \mathrm{~Hz}, 2 \mathrm{~F}),-157.8(\mathrm{dd}, J=9.0,21.1 \mathrm{~Hz}, 2 \mathrm{~F}) ; \mathrm{HRMS}(\mathrm{ESI}) \mathrm{m} / \mathrm{z}: \quad\left[\mathrm{M}+\mathrm{NH}_{4}\right]^{+}$calcd for $\mathrm{C}_{23} \mathrm{H}_{21} \mathrm{~F}_{5} \mathrm{NO}_{4} \mathrm{~S}$ 502.1106; found 502.1105.

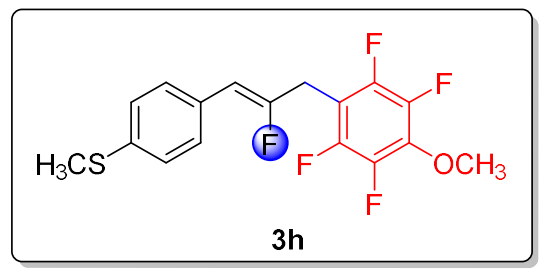

(Z)-(4-(2-fluoro-3-(2,3,5,6-tetrafluoro-4-methoxyphenyl)prop-1-en-1-yl)phenyl)(methyl)sulfa

ne: $\mathrm{Rf}=0.29$ (peterolium ether/ethyl acetate, 100:1); Column chromatoghraphy(silica gel; peterolium ether/ethyl acetate, 200:1); 94.0mg, 87\%; white solid; ${ }^{1} \mathrm{H}$ NMR $\left(400 \mathrm{MHz}, \mathrm{CDCl}_{3}\right) \delta$ : $7.27(\mathrm{~d}, J=8.4 \mathrm{~Hz}, 2 \mathrm{H}), 7.08(\mathrm{~d}, J=8.4 \mathrm{~Hz}, 2 \mathrm{H}), 5.42(\mathrm{~d}, J=38.4 \mathrm{~Hz}, 1 \mathrm{H}), 3.98(\mathrm{~s}, 3 \mathrm{H}), 3.60(\mathrm{~d}$, $J=15.6 \mathrm{~Hz}, 2 \mathrm{H}), 2.37(\mathrm{~s}, 3 \mathrm{H}) ;{ }^{13} \mathrm{C} \mathrm{NMR}\left(100 \mathrm{MHz}, \mathrm{CDCl}_{3}\right) \delta: 156.8,154.1,145.5(\mathrm{dm}, J=248.9 \mathrm{~Hz})$, $140.9(\mathrm{dm}, J=245.8 \mathrm{~Hz}), \quad 137.5(\mathrm{~d}, J=2.7 \mathrm{~Hz}), 129.6(\mathrm{~d}, J=2.9 \mathrm{~Hz}), 128.7(\mathrm{~d}, J=7.5 \mathrm{~Hz}), \quad 126.2$, $107.7(\mathrm{~m}), 106.9(\mathrm{~d}, J=7.7 \mathrm{~Hz}), 62.1(\mathrm{t}, J=3.6 \mathrm{~Hz}), 26.1(\mathrm{~d}, J=30.7 \mathrm{~Hz}), 15.5 ;{ }^{19} \mathrm{~F}$ NMR $(376 \mathrm{MHz}$, $\left.\mathrm{CDCl}_{3}\right) \delta:-102.8(\mathrm{~m}, 1 \mathrm{~F}),-144.7(\mathrm{~m}, 2 \mathrm{~F}),-157.9(\mathrm{dd}, J=7.9,20.3 \mathrm{~Hz}, 2 \mathrm{~F}) ; \mathrm{HRMS}(\mathrm{ESI}) \mathrm{m} / \mathrm{z}:[\mathrm{M}+\mathrm{H}]^{+}$ 
calcd for $\mathrm{C}_{17} \mathrm{H}_{14} \mathrm{~F}_{5} \mathrm{OS} 361.0680$; found 361.0681 .

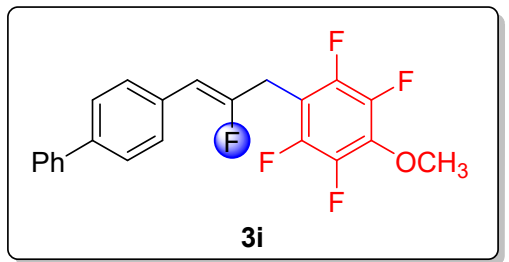

(Z)-4-(2-fluoro-3-(2,3,5,6-tetrafluoro-4-methoxyphenyl)prop-1-en-1-yl)-1,1'-biphenyl;

$\mathrm{Rf}=0.30$ (peterolium ether/ethyl acetate, 100:1); Column chromatoghraphy(silica gel; peterolium ether/ethyl acetate, 200:1); 94.8mg, 81\%; white solid; ${ }^{1} \mathrm{H}$ NMR (400MHz, $\left.\mathrm{CDCl}_{3}\right) \delta: 7.59-7.50(\mathrm{~m}$, $6 \mathrm{H}), 7.44-7.40(\mathrm{~m}, 2 \mathrm{H}), 7.35-7.31(\mathrm{~m}, 1 \mathrm{H}), 5.60(\mathrm{~d}, J=38.4 \mathrm{~Hz}, 1 \mathrm{H}), 4.07(\mathrm{~s}, 3 \mathrm{H}), 3.72(\mathrm{~d}, J=15.6 \mathrm{~Hz}$, $2 \mathrm{H}) ;{ }^{13} \mathrm{C} \mathrm{NMR}\left(100 \mathrm{MHz}, \mathrm{CDCl}_{3}\right) \delta: 157.2,154.5,145.6(\mathrm{dm}, J=257.4 \mathrm{~Hz}), 140.9(\mathrm{dm}, J=246.3 \mathrm{~Hz})$, $140.5,139.9(\mathrm{~d}, J=2.4 \mathrm{~Hz}), 131.9(\mathrm{~d}, J=3.0 \mathrm{~Hz}), 128.8(\mathrm{~d}, J=7.4 \mathrm{~Hz}), 128.7,127.3,127.0,126.9$, $107.7(\mathrm{t}, J=18.2 \mathrm{~Hz}), 107.1(\mathrm{~d}, J=7.6 \mathrm{~Hz}), 62.1(\mathrm{t}, J=3.6 \mathrm{~Hz}), 26.2(\mathrm{~d}, J=30.7 \mathrm{~Hz}) ;{ }^{19} \mathrm{~F}$ NMR $(376 \mathrm{MHz}$, $\left.\mathrm{CDCl}_{3}\right) \delta:-102.2(\mathrm{~m}, 1 \mathrm{~F}),-144.5(\mathrm{dd}, J=5.3,12.8 \mathrm{~Hz}, 2 \mathrm{~F}),-157.9(\mathrm{~m}, 2 \mathrm{~F}) ; \mathrm{HRMS}(\mathrm{APCI}) \mathrm{m} / \mathrm{z}$ : $[\mathrm{M}+\mathrm{H}]^{+}$calcd for $\mathrm{C}_{22} \mathrm{H}_{16} \mathrm{~F}_{5} \mathrm{O} 391.1116$; found 391.1119.

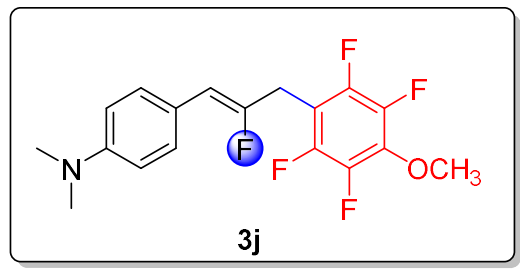

(Z)-4-(2-fluoro-3-(2,3,5,6-tetrafluoro-4-methoxyphenyl)prop-1-en-1-yl)-N,N-dimethylaniline: $\mathrm{Rf}=0.32$ (peterolium ether/ethyl acetate, 30:1); Column chromatoghraphy (silica gel; peterolium ether/ethyl acetate, 70:1); 83.5mg, 78\%; white solid; ${ }^{1} \mathrm{H}$ NMR $\left(400 \mathrm{MHz}, \mathrm{CDCl}_{3}\right) \delta$ : $7.25(\mathrm{~d}$, $J=8.8 \mathrm{~Hz}, 2 \mathrm{H}), 6.57(\mathrm{~d}, J=8.8 \mathrm{~Hz}, 2 \mathrm{H}), 5.38(\mathrm{~d}, J=39.2 \mathrm{~Hz}, 1 \mathrm{H}), 3.98(\mathrm{~s}, 3 \mathrm{H}), 3.59(\mathrm{~d}, J=16.0 \mathrm{~Hz}, 2 \mathrm{H})$, $2.86(\mathrm{~s}, 6 \mathrm{H}) ;{ }^{13} \mathrm{C} \mathrm{NMR}\left(100 \mathrm{MHz}, \mathrm{CDCl}_{3}\right) \delta: 154.4,151.8,149.5(\mathrm{~d}, J=2.1 \mathrm{~Hz}), 145.5(\mathrm{dm}$, $J=232.1 \mathrm{~Hz}), 140.9(\mathrm{dm}, J=245.6 \mathrm{~Hz}), 129.3(\mathrm{~d}, J=7.3 \mathrm{~Hz}), 121.2,112.1,108.4(\mathrm{~m}), 107.2(\mathrm{~d}$, $J=8.2 \mathrm{~Hz}), 62.1(\mathrm{t}, J=3.5 \mathrm{~Hz}), 40.3,26.1(\mathrm{~d}, J=30.8 \mathrm{~Hz}) ;{ }^{19} \mathrm{~F} \mathrm{NMR}\left(376 \mathrm{MHz}, \mathrm{CDCl}_{3}\right) \delta:-107.7(\mathrm{~m}$, $1 \mathrm{~F}),-144.7(\mathrm{~m}, 2 \mathrm{~F}),-158.1(\mathrm{~m}, 2 \mathrm{~F})$; HRMS(ESI) $\mathrm{m} / \mathrm{z}$ : $[\mathrm{M}+\mathrm{H}]^{+}$calcd for $\mathrm{C}_{18} \mathrm{H}_{17} \mathrm{~F}_{5} \mathrm{NO} 358.1225$; found 358.1225 .

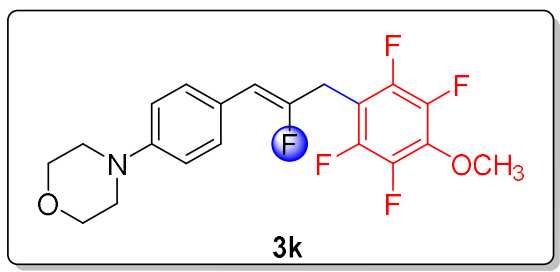

(Z)-4-(4-(2-fluoro-3-(2,3,5,6-tetrafluoro-4-methoxyphenyl)prop-1-en-1-yl)phenyl)morpholine; $\mathrm{Rf}=0.28$ (peterolium ether/ethyl acetate, 10:1); Column chromatoghraphy( silica gel; peterolium ether/ethyl acetate, 20:1); 73.0mg, 61\%; white solid; ${ }^{1} \mathrm{H}$ NMR $\left(400 \mathrm{MHz}, \mathrm{CDCl}_{3}\right) \delta: 7.36(\mathrm{~d}, J$ $=8.8 \mathrm{~Hz}, 2 \mathrm{H}), 6.83(\mathrm{~d}, J=8.8 \mathrm{~Hz}, 2 \mathrm{H}), 5.48(\mathrm{~d}, J=38.8 \mathrm{~Hz}, 1 \mathrm{H}), 4.06(\mathrm{~s}, 3 \mathrm{H}), 3.85(\mathrm{t}, J=4.8 \mathrm{~Hz}, 4 \mathrm{H})$, $3.67(\mathrm{~d}, J=16.0 \mathrm{~Hz}, 2 \mathrm{H}), 3.15(\mathrm{t}, J=4.8 \mathrm{~Hz}, 4 \mathrm{H}) ;{ }^{13} \mathrm{C} \mathrm{NMR}\left(100 \mathrm{MHz}, \mathrm{CDCl}_{3}\right) \delta: 155.4,152.8,150.0$, $145.4(\mathrm{dm}, J=244.7 \mathrm{~Hz}), 140.9(\mathrm{dm}, J=245.8 \mathrm{~Hz}), 129.3(\mathrm{~d}, J=7.3 \mathrm{~Hz}), 124.5,115.1,108.1(\mathrm{~m})$, 106.9(d, $J=8.1 \mathrm{~Hz}), 66.7,62.1(\mathrm{t}, J=3.6 \mathrm{~Hz}), 48.8,26.1(\mathrm{~d}, J=30.7 \mathrm{~Hz}) ;{ }^{19} \mathrm{~F}$ NMR $\left(376 \mathrm{MHz}, \mathrm{CDCl}_{3}\right) \delta$ : $-105.8(\mathrm{~m}, 1 \mathrm{~F}),-144.7(\mathrm{dd}, J=5.3,12.7 \mathrm{~Hz}, 2 \mathrm{~F}),-158.0(\mathrm{~m}, 2 \mathrm{~F}) ; \mathrm{HRMS}(\mathrm{APCI}) \mathrm{m} / \mathrm{z}:[\mathrm{M}+\mathrm{H}]^{+}$calcd 
for $\mathrm{C}_{20} \mathrm{H}_{19} \mathrm{~F}_{5} \mathrm{NO}_{2} 400.1330$; found 400.1328 .

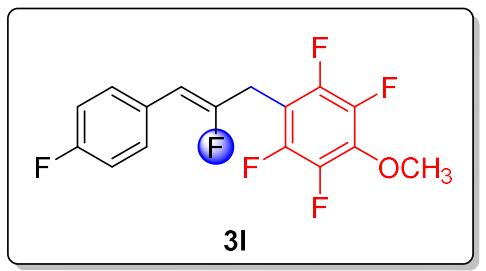

\section{(Z)-1,2,4,5-tetrafluoro-3-(2-fluoro-3-(4-fluorophenyl)allyl)-6-methoxybenzene;}

$\mathrm{Rf}=0.33$ (peterolium ether/ethyl acetate, 100:1); Column chromatoghraphy(silica gel; peterolium ether/ethyl acetate, 200:1); 83.6mg, 84\%; colourless oil; ${ }^{1} \mathrm{H} \mathrm{NMR}\left(400 \mathrm{MHz}, \mathrm{CDCl}_{3}\right) \delta: 7.41(\mathrm{dd}$, $J=5.6,8.8 \mathrm{~Hz}, 2 \mathrm{H}), 7.00(\mathrm{t}, J=8.8 \mathrm{~Hz}, 2 \mathrm{H}), 5.53(\mathrm{~d}, J=37.6 \mathrm{~Hz}, 1 \mathrm{H}), 4.08(\mathrm{~s}, 3 \mathrm{H}), 3.69(\mathrm{~d}, J=15.6 \mathrm{~Hz}$, $2 \mathrm{H}) ;{ }^{13} \mathrm{C}$ NMR $\left(100 \mathrm{MHz}, \mathrm{CDCl}_{3}\right) \delta: 161.7(\mathrm{~m}), 156.7,154.0,145.5(\mathrm{dm}, J=249.4 \mathrm{~Hz}), 141.0(\mathrm{dm}$, $J=245.8 \mathrm{~Hz}), 130.1(\mathrm{t}, J=7.7 \mathrm{~Hz}), 129.0(\mathrm{~m}), 115.3(\mathrm{~d}, J=21.3 \mathrm{~Hz}), 107.6(\mathrm{~m}), 106.4(\mathrm{~d}, J=7.8 \mathrm{~Hz})$, 62.1(t, $J=3.7 \mathrm{~Hz}), 26.1(\mathrm{~d}, J=30.7 \mathrm{~Hz}) ;{ }^{19} \mathrm{~F}$ NMR $\left(376 \mathrm{MHz}, \mathrm{CDCl}_{3}\right) \delta:-103.9(\mathrm{~m}, 1 \mathrm{~F}),-114.0(\mathrm{~m}, 1 \mathrm{~F})$, $-144.6(\mathrm{dd}, J=5.3,12.4 \mathrm{~Hz}, 2 \mathrm{~F}),-157.9(\mathrm{~m}, 2 \mathrm{~F}) ; \operatorname{HRMS}(\mathrm{APCI}) \mathrm{m} / \mathrm{z}:[\mathrm{M}]^{+}$calcd for $\mathrm{C}_{16} \mathrm{H}_{10} \mathrm{~F}_{6} \mathrm{O}$ 332.0636; found 332.0633 .

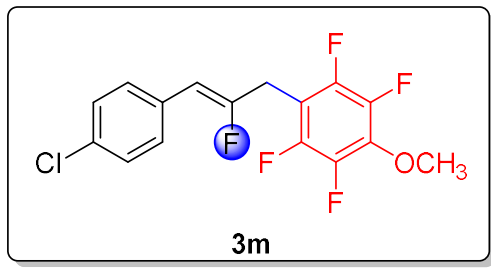

\section{(Z)-1-(3-(4-chlorophenyl)-2-fluoroallyl)-2,3,5,6-tetrafluoro-4-methoxybenzene;}

$\mathrm{Rf}=0.31$ (peterolium ether/ethyl acetate, 100:1); Column chromatoghraphy(silica gel; peterolium ether/ethyl acetate, 200:1); 39.7mg, 38\%; colourless oil; ${ }^{1} \mathrm{H}$ NMR $\left(400 \mathrm{MHz}, \mathrm{CDCl}_{3}\right) \delta: 7.37(\mathrm{~d}$, $J=8.8 \mathrm{~Hz}, 2 \mathrm{H}), 7.27(\mathrm{~d}, J=8.4 \mathrm{~Hz}, 2 \mathrm{H}), 5.52(\mathrm{~d}, J=37.6 \mathrm{~Hz}, 1 \mathrm{H}), 4.08(\mathrm{~s}, 3 \mathrm{H}), 3.70(\mathrm{~d}, J=15.2 \mathrm{~Hz}, 2 \mathrm{H})$; ${ }^{13} \mathrm{C}$ NMR $\left(100 \mathrm{MHz}, \mathrm{CDCl}_{3}\right) \delta: 157.5,154.8,145.5(\mathrm{dm}, J=244.9 \mathrm{~Hz}), 140.9(\mathrm{dm}, J=245.8 \mathrm{~Hz})$, $132.1(\mathrm{~m}), 129.6(\mathrm{~d}, J=7.6 \mathrm{~Hz}), 128.6,115.3(\mathrm{~d}, J=21.3 \mathrm{~Hz}), 107.5(\mathrm{~m}), 106.4(\mathrm{~d}, J=7.6 \mathrm{~Hz}), 62.1(\mathrm{t}$, $J=3.7 \mathrm{~Hz}), 26.1(\mathrm{~d}, J=30.5 \mathrm{~Hz}) ;{ }^{19} \mathrm{~F}$ NMR $\left(376 \mathrm{MHz}, \mathrm{CDCl}_{3}\right) \delta:-101.7(\mathrm{t}, J=4.9 \mathrm{~Hz}, 1 \mathrm{~F}),-144.6(\mathrm{~m}$, 2F), -157.8(m, 2F); HRMS(APCI) m/z: [M] calcd for $\mathrm{C}_{16} \mathrm{H}_{10} \mathrm{ClF}_{5} \mathrm{O} 348.0340$; found 348.0335.

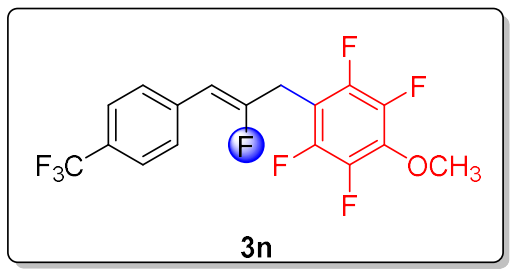

\section{(Z)-1,2,4,5-tetrafluoro-3-(2-fluoro-3-(4-(trifluoromethyl)phenyl)allyl)-6-methoxybenzene:}

$\mathrm{Rf}=0.33$ (peterolium ether/ethyl acetate, 100:1); Column chromatoghraphy(silica gel; peterolium ether/ethyl acetate, 200:1); 98.5mg, 86\%; Z/E=10:1; yellow solid; ${ }^{1} \mathrm{H}$ NMR $\left(400 \mathrm{MHz}, \mathrm{CDCl}_{3}\right) \delta$ : 7.54(s, $4 \mathrm{H}), 5.61(\mathrm{~d}, J=37.2 \mathrm{~Hz}, 1 \mathrm{H}), 4.08(\mathrm{~s}, 3 \mathrm{H}), 3.73(\mathrm{~d}, J=15.2 \mathrm{~Hz}, 2 \mathrm{H}) ;{ }^{13} \mathrm{C}$ NMR $(100 \mathrm{MHz}$, $\left.\mathrm{CDCl}_{3}\right) \delta: 158.8,156.1,145.6(\mathrm{dm}, J=245.0 \mathrm{~Hz}), 140.9(\mathrm{dm}, J=246.0 \mathrm{~Hz}), 136.3,129.2,128.5(\mathrm{~d}$, $J=7.6 \mathrm{~Hz}), 125.3(\mathrm{q}, J=3.8 \mathrm{~Hz}), 122.7,107.2(\mathrm{~m}), 106.4(\mathrm{~d}, J=7.4 \mathrm{~Hz}), 62.1(\mathrm{t}, J=3.6 \mathrm{~Hz}), 26.1(\mathrm{~d}$, $J=30.4 \mathrm{~Hz}) ;{ }^{19} \mathrm{~F}$ NMR $\left(376 \mathrm{MHz}, \mathrm{CDCl}_{3}\right) \delta:-62.7(\mathrm{~s}, 3 \mathrm{~F}),-99.3(\mathrm{~m}, 1 \mathrm{~F}),-144.6(\mathrm{~m}, 2 \mathrm{~F}),-157.7(\mathrm{~m}$, 2F); HRMS(ESI) m/z: [M+H] calcd for $\mathrm{C}_{17} \mathrm{H}_{11} \mathrm{~F}_{8} \mathrm{O} 383.0677$; found 383.0688 . 


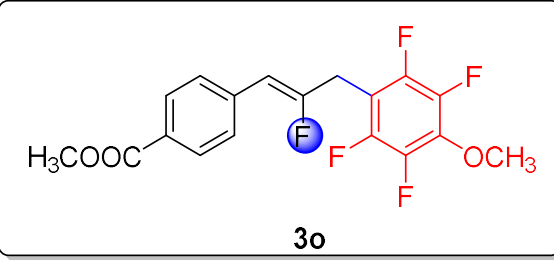

methyl(Z)-4-(2-fluoro-3-(2,3,5,6-tetrafluoro-4-methoxyphenyl)prop-1-en-1-yl)benzoate;

$\mathrm{Rf}=0.32$ (peterolium ether/ethyl acetate, 30:1); Column chromatoghraphy(silica gel; peterolium ether/ethyl acetate, 60:1); 73.7mg, 66\%; white solid; ${ }^{1} \mathrm{H}$ NMR $\left(400 \mathrm{MHz}, \mathrm{CDCl}_{3}\right) \delta: 7.96(\mathrm{~d}$, $J=8.4 \mathrm{~Hz}, 2 \mathrm{H}), 7.50(\mathrm{~d}, J=8.4 \mathrm{~Hz}, 2 \mathrm{H}), 5.62(\mathrm{~d}, J=37.6 \mathrm{~Hz}, 1 \mathrm{H}), 4.08(\mathrm{~s}, 3 \mathrm{H}), 3.90(\mathrm{~s}, 3 \mathrm{H}), 3.73(\mathrm{~d}$, $J=15.6 \mathrm{~Hz}, 2 \mathrm{H}) ;{ }^{13} \mathrm{C}$ NMR $\left(100 \mathrm{MHz}, \mathrm{CDCl}_{3}\right) \delta: 166.7,158.7,145.5(\mathrm{dm}, J=249.4 \mathrm{~Hz}), 140.9(\mathrm{dm}$, $J=246.1 \mathrm{~Hz}), 137.4(\mathrm{~d}, J=2.9 \mathrm{~Hz}), 129.6,128.5(\mathrm{~d}, J=2.4 \mathrm{~Hz}), 128.2(\mathrm{~d}, J=7.7 \mathrm{~Hz}), 107.2(\mathrm{~m}), 106.8(\mathrm{~d}$, $J=7.2 \mathrm{~Hz}), 62.0(\mathrm{t}, J=3.6 \mathrm{~Hz}), 52.0,26.1(\mathrm{~d}, J=30.4 \mathrm{~Hz}) ;{ }^{19} \mathrm{~F} \mathrm{NMR}\left(376 \mathrm{MHz}, \mathrm{CDCl}_{3}\right) \delta:-98.8(\mathrm{~m}, 1 \mathrm{~F})$, $-144.5(\mathrm{dd}, J=4.9,12.8 \mathrm{~Hz}, 2 \mathrm{~F}),-157.8(\mathrm{dd}, J=8.6,21.4 \mathrm{~Hz}, 2 \mathrm{~F})$; HRMS(ESI) $\mathrm{m} / \mathrm{z}:[\mathrm{M}+\mathrm{H}]^{+}$calcd for $\mathrm{C}_{18} \mathrm{H}_{14} \mathrm{~F}_{5} \mathrm{O}_{3} 373.0858$; found 373.0856 .

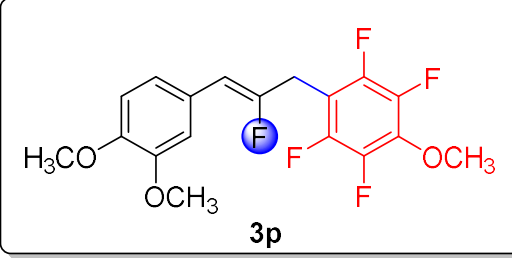

\section{(Z)-1-(3-(3,4-dimethoxyphenyl)-2-fluoroallyl)-2,3,5,6-tetrafluoro-4-methoxybenzene:}

$\mathrm{Rf}=0.26$ (peterolium ether/ethyl acetate, 10:1); Column chromatoghraphy(silica gel; peterolium ether/ethyl acetate, 20:1); 94.2mg, 84\%; white solid; ${ }^{1} \mathrm{H}$ NMR (400 MHz, $\left.\mathrm{CDCl}_{3}\right) \delta: 7.07(\mathrm{~s}, 1 \mathrm{H})$, $6.95(\mathrm{dd}, J=2.0 \mathrm{~Hz}, 8.4 \mathrm{~Hz}, 1 \mathrm{H}), 6.80(\mathrm{~d}, J=8.4 \mathrm{~Hz}, 1 \mathrm{H}), 5.50(\mathrm{~d}, J=38.4 \mathrm{~Hz}, 1 \mathrm{H}), 4.07(\mathrm{~s}, 3 \mathrm{H}), 3.86(\mathrm{~d}$, $J=1.6 \mathrm{~Hz}, 6 \mathrm{H}), 3.69(\mathrm{~d}, J=15.6 \mathrm{~Hz}, 2 \mathrm{H}) ;{ }^{13} \mathrm{C} \mathrm{NMR}\left(100 \mathrm{MHz}, \mathrm{CDCl}_{3}\right) \delta: 155.7,153.1,148.6$, $148.2(\mathrm{~d}, J=2.7 \mathrm{~Hz}), 145.5(\mathrm{dm}, J=244.6 \mathrm{~Hz}), 140.9(\mathrm{dm}, J=246.0 \mathrm{~Hz}), 125.7(\mathrm{~d}, J=2.9 \mathrm{~Hz}), 121.3(\mathrm{~d}$, $J=6.5 \mathrm{~Hz}), 111.2(\mathrm{~d}, J=8.9 \mathrm{~Hz}), 110.8,107.9(\mathrm{~m}), 107.1(\mathrm{~d}, J=7.6 \mathrm{~Hz}), 62.0(\mathrm{t}, J=3.6 \mathrm{~Hz}), 55.7,55.6$, 26.1(d, $J=30.7 \mathrm{~Hz}) ;{ }^{19} \mathrm{~F}$ NMR $\left(376 \mathrm{MHz}, \mathrm{CDCl}_{3}\right) \delta:-105.4(\mathrm{~m}, 1 \mathrm{~F}),-144.7(\mathrm{~m}, 2 \mathrm{~F}),-158.0(\mathrm{~m}, 2 \mathrm{~F})$; HRMS(ESI) m/z: [M+H] calcd for $\mathrm{C}_{18} \mathrm{H}_{16} \mathrm{~F}_{5} \mathrm{O}_{3}$ 375.1014; found 375.1006 .

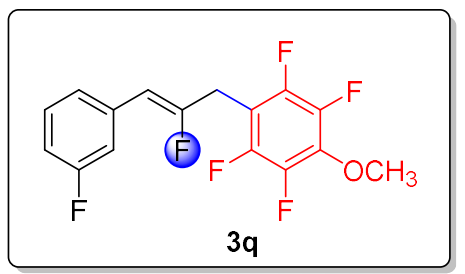

\section{(Z)-1,2,4,5-tetrafluoro-3-(2-fluoro-3-(3-fluorophenyl)allyl)-6-methoxybenzene;}

$\mathrm{Rf}=0.33$ (peterolium ether/ethyl acetate, 100:1); Column chromatoghraphy(silica gel; peterolium ether/ethyl acetate, 200:1); 92.6mg, Z/E=18:1; 93\%; colourless oil; ${ }^{1} \mathrm{H}$ NMR (400MHz, $\left.\mathrm{CDCl}_{3}\right) \delta$ : $7.29-7.15(\mathrm{~m}, 3 \mathrm{H}), 6.94-6.90(\mathrm{~m}, 1 \mathrm{H}), 5.55(\mathrm{~d}, J=37.2 \mathrm{~Hz}, 1 \mathrm{H}), 4.08(\mathrm{~s}, 3 \mathrm{H}), 3.71(\mathrm{~d}, J=15.2 \mathrm{~Hz}, 2 \mathrm{H})$; ${ }^{13} \mathrm{C}$ NMR $\left(100 \mathrm{MHz}, \mathrm{CDCl}_{3}\right) \delta: 162.7(\mathrm{~d}, J=243.3 \mathrm{~Hz}), 157.9,155.3,145.5(\mathrm{dm}, J=245.0 \mathrm{~Hz})$, $140.9(\mathrm{dm}, J=246.7 \mathrm{~Hz}), 134.8(\mathrm{~m}), 129.7(\mathrm{~d}, J=8.4 \mathrm{~Hz}), 124.2(\mathrm{~m}), 115.1(\mathrm{dd}, J=8.7,22.6 \mathrm{~Hz})$, $114.1(\mathrm{dd}, J=8.0,21.2 \mathrm{~Hz}), 107.4(\mathrm{t}, J=18.2 \mathrm{~Hz}), 106.6(\mathrm{~d}, J=7.4 \mathrm{~Hz}), 62.1(\mathrm{t}, J=3.6 \mathrm{~Hz}), 26.1(\mathrm{~d}$, $J=30.6 \mathrm{~Hz}) ;{ }^{19} \mathrm{~F}$ NMR $\left(376 \mathrm{MHz}, \mathrm{CDCl}_{3}\right) \delta:-100.6(\mathrm{~m}, 1 \mathrm{~F}),-133.2(\mathrm{~m}, 1 \mathrm{~F}),-144.6(\mathrm{dd}, J=4.9$, 
12.4Hz, 2F), 157.8(m, 2F); HRMS(APCI) $\mathrm{m} / \mathrm{z}$ : $[\mathrm{M}]^{+}$calcd for $\mathrm{C}_{16} \mathrm{H}_{10} \mathrm{~F}_{6} \mathrm{O}$ 332.0636; found 332.0630 .

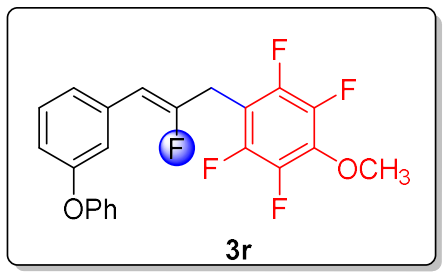

(Z)-1,2,4,5-tetrafluoro-3-(2-fluoro-3-(3-phenoxyphenyl)allyl)-6-methoxybenzene:

$\mathrm{Rf}=0.27$ (peterolium ether/ethyl acetate, 100:1); Column chromatoghraphy(silica gel; peterolium ether/ethyl acetate, 200:1); 114.5mg, 94\%; yellow oil; ${ }^{1} \mathrm{H}$ NMR $\left(400 \mathrm{MHz}, \mathrm{CDCl}_{3}\right) \delta: 7.25-715(\mathrm{~m}$, $3 \mathrm{H}), 7.09(\mathrm{~d}, J=8.0 \mathrm{~Hz}, 1 \mathrm{H}), 7.03-6.98(\mathrm{~m}, 2 \mathrm{H}), 6.91-6.89(\mathrm{~m}, 2 \mathrm{H}), 6.80-6.78(\mathrm{~m}, 1 \mathrm{H}), 5.43(\mathrm{~d}$, $J=37.6 \mathrm{~Hz}, 1 \mathrm{H}), 3.97(\mathrm{~s}, 3 \mathrm{H}), 3.58(\mathrm{~d}, J=15.6 \mathrm{~Hz}, 2 \mathrm{H}) ;{ }^{13} \mathrm{C} \mathrm{NMR}\left(100 \mathrm{MHz}, \mathrm{CDCl}_{3}\right) \delta: 157.5,157.1$, $154.8,145.6(\mathrm{dm}, J=244.9 \mathrm{~Hz}), 140.9(\mathrm{dm}, J=246.0 \mathrm{~Hz}), 134.5(\mathrm{~d}, J=2.7 \mathrm{~Hz}), 129.6(\mathrm{~d}, J=2.3 \mathrm{~Hz})$, $123.5,123.4,123.1,118.9,118.8,118.7,117.9(\mathrm{~d}, J=1.9 \mathrm{~Hz}), 107.5(\mathrm{~m}), 106.9(\mathrm{~d}, J=7.3 \mathrm{~Hz}), 62.1(\mathrm{t}$, $J=3.6 \mathrm{~Hz}), 26.1(\mathrm{~d}, J=30.5 \mathrm{~Hz}) ;{ }^{19} \mathrm{~F}$ NMR $\left(376 \mathrm{MHz}, \mathrm{CDCl}_{3}\right) \delta:-101.2(\mathrm{t}, J=4.9 \mathrm{~Hz}, 1 \mathrm{~F}),-144.5(\mathrm{~m}$, $2 \mathrm{~F}$ ), $-157.8(\mathrm{dd}, J=7.9,20.6 \mathrm{~Hz}, 2 \mathrm{~F})$; HRMS(ESI) $\mathrm{m} / \mathrm{z}$ : $[\mathrm{M}+\mathrm{H}]^{+}$calcd for $\mathrm{C}_{22} \mathrm{H}_{16} \mathrm{~F}_{5} \mathrm{O}_{2} 407.1065$; found 407.1064 .

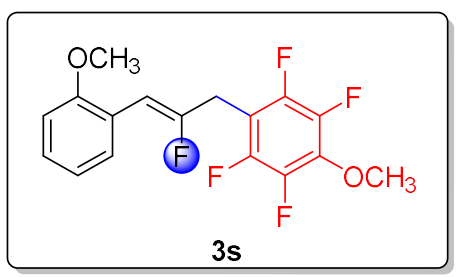

(Z)-1,2,4,5-tetrafluoro-3-(2-fluoro-3-(2-methoxyphenyl)allyl)-6-methoxybenzene:

$\mathrm{Rf}=0.28$ (peterolium ether/ethyl acetate, 100:1); Column chromatoghraphy(silica gel; peterolium ether/ethyl acetate, 200:1); 84.6mg, 82\%; colorless oil; ${ }^{1} \mathrm{H}$ NMR $\left(400 \mathrm{MHz}, \mathrm{CDCl}_{3}\right) \delta: 7.61(\mathrm{dd}$, $J=1.6,8.0 \mathrm{~Hz}, 1 \mathrm{H}), 7.16-7.09(\mathrm{~m}, 1 \mathrm{H}), 6.83(\mathrm{t}, J=7.6 \mathrm{~Hz}, 1 \mathrm{H}), 6.75(\mathrm{~d}, J=8.4 \mathrm{~Hz}, 1 \mathrm{H}), 5.96(\mathrm{~d}$, $J=39.6 \mathrm{~Hz}, 1 \mathrm{H}), 3.98(\mathrm{~s}, 3 \mathrm{H}), 3.73(\mathrm{~s}, 3 \mathrm{H}), 3.63(\mathrm{~d}, J=16.8 \mathrm{~Hz}, 2 \mathrm{H}) ;{ }^{13} \mathrm{C} \mathrm{NMR}\left(100 \mathrm{MHz}, \mathrm{CDCl}_{3}\right) \delta$ : $156.8,155.9,154.1,145.6(\mathrm{dm}, J=244.8 \mathrm{~Hz}), 140.9(\mathrm{dm}, J=245.6 \mathrm{~Hz}), 129.6(\mathrm{~d}, J=12.6 \mathrm{~Hz}), 128.4(\mathrm{~d}$, $J=1.7 \mathrm{~Hz}), 121.5(\mathrm{~d}, J=3.3 \mathrm{~Hz}), 120.5,110.3,108.1(\mathrm{~m}), 101.0(\mathrm{~d}, J=6.0 \mathrm{~Hz}), 62.1(\mathrm{t}, J=3.6 \mathrm{~Hz}), 55.4$, 26.5(d, $J=30.5 \mathrm{~Hz}) ;{ }^{19} \mathrm{~F}$ NMR $\left(376 \mathrm{MHz}, \mathrm{CDCl}_{3}\right) \delta$ : $-104.9(\mathrm{~m}, 1 \mathrm{~F}),-144.5(\mathrm{~m}, 2 \mathrm{~F}),-158.1(\mathrm{~m}, 2 \mathrm{~F})$; HRMS(ESI) m/z: [M+Na] calcd for $\mathrm{C}_{17} \mathrm{H}_{13} \mathrm{~F}_{5} \mathrm{O}_{2} \mathrm{Na}$ 367.0728; found 367.0727 .

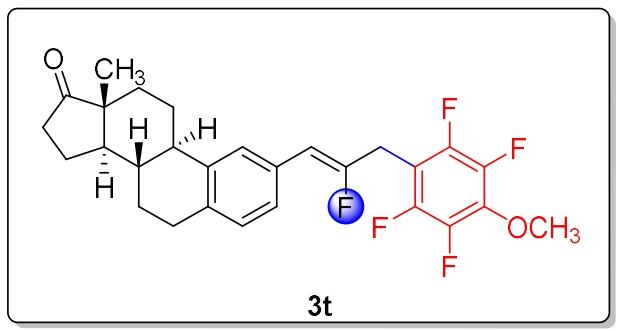

(8S,9R,13R,14R)-2-((Z)-2-fluoro-3-(2,3,5,6-tetrafluoro-4-methoxyphenyl)prop-1-en-1-yl)-13methyl-6,7,8,9,11,12,13,14,15,16-decahydro-17H-cyclopenta[a]phenanthren-17-one;

$\mathrm{Rf}=0.26$ (peterolium ether/ethyl acetate, 10:1); Column chromatoghraphy(silica gel; peterolium ether/ethyl acetate, 30:1); 102.9mg, 70\%; white solid; ${ }^{1} \mathrm{H}$ NMR $\left(400 \mathrm{MHz}, \mathrm{CDCl}_{3}\right) \delta: 7.23(\mathrm{~s}, 2 \mathrm{H})$, 
7.18(s, $1 \mathrm{H}), 5.51(\mathrm{~d}, J=38.4 \mathrm{~Hz}, 1 \mathrm{H}), 4.07(\mathrm{~s}, 3 \mathrm{H}), 3.68(\mathrm{~d}, J=15.6 \mathrm{~Hz}, 2 \mathrm{H}), 2.89-2.86(\mathrm{~m}, 2 \mathrm{H})$, 2.51-2.46(m, $1 \mathrm{H}), 2.43-2.38(\mathrm{~m}, 1 \mathrm{H}), 2.28-2.23(\mathrm{~m}, 1 \mathrm{H}), 2.16-1.93(\mathrm{~m}, 4 \mathrm{H}), 1.62-1.46(\mathrm{~m}, 6 \mathrm{H})$, $0.90(\mathrm{~s}, 3 \mathrm{H}) ;{ }^{13} \mathrm{C} \mathrm{NMR}\left(100 \mathrm{MHz}, \mathrm{CDCl}_{3}\right) \delta: 220.8,156.6,154.0,145.4(\mathrm{dm}, J=244.9 \mathrm{~Hz}), 140.9(\mathrm{dm}$, $J=245.7 \mathrm{~Hz}), 138.9(\mathrm{~d}, J=2.0 \mathrm{~Hz}), 136.4,130.3(\mathrm{~d}, J=2.7 \mathrm{~Hz}), 128.9(\mathrm{~d}, J=7.1 \mathrm{~Hz}), 125.8(\mathrm{~d}, J=7.1 \mathrm{~Hz})$, 125.4, 107.9(m), 107.1(d, $J=7.8 \mathrm{~Hz}), 62.1(\mathrm{t}, J=3.6 \mathrm{~Hz}), 50.4,47.9,44.3,38.0,35.8,31.5,29.3,26.4$, $26.1(\mathrm{~d}, J=31.0 \mathrm{~Hz}), 25.6,21.5,13.7 ;{ }^{19} \mathrm{~F}$ NMR $\left(376 \mathrm{MHz}, \mathrm{CDCl}_{3}\right) \delta:-103.4(\mathrm{~m}, 1 \mathrm{~F}),-144.6(\mathrm{dd}$, $J=4.9,12.8 \mathrm{~Hz}, 2 \mathrm{~F}),-158.0(\mathrm{~m}, 2 \mathrm{~F})$; HRMS(APCI) $\mathrm{m} / \mathrm{z}:[\mathrm{M}+\mathrm{H}]^{+}$calcd for $\mathrm{C}_{28} \mathrm{H}_{28} \mathrm{~F}_{5} \mathrm{O}_{2} 491.2004$; found 491.2005 .

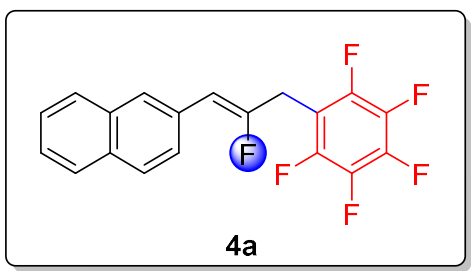

(Z)-2-(2-fluoro-3-(perfluorophenyl)prop-1-en-1-yl)naphthalene; $\mathrm{Rf}=0.40$ (peterolium ether); Column chromatoghraphy(silica gel; peterolium ether); $95.6 \mathrm{mg}$, 90\%; white solid; ${ }^{1} \mathrm{H}$ NMR $\left(400 \mathrm{MHz}, \mathrm{CDCl}_{3}\right) \delta: 7.84(\mathrm{~s}, 1 \mathrm{H}), 7.77-7.74(\mathrm{~m}, 3 \mathrm{H}), 7.59(\mathrm{dd}, J=1.6,8.4 \mathrm{~Hz}, 1 \mathrm{H}), 7.44-7.42(\mathrm{~m}, 2 \mathrm{H})$, $5.72(\mathrm{~d}, J=38.0 \mathrm{~Hz}, 1 \mathrm{H}), 3.73(\mathrm{~d}, J=16.0 \mathrm{~Hz}, 2 \mathrm{H}) ;{ }^{13} \mathrm{C} \mathrm{NMR}\left(100 \mathrm{MHz}, \mathrm{CDCl}_{3}\right) \delta: 156.6,153.9$, $145.3(\mathrm{dm}, J=253.9 \mathrm{~Hz}), 140.6(\mathrm{dm}, J=265.3 \mathrm{~Hz}), 137.6(\mathrm{dm}, J=263.7 \mathrm{~Hz}), 133.3,132.4(\mathrm{~d}, J=1.6 \mathrm{~Hz})$, $130.2(\mathrm{~d}, J=3.1 \mathrm{~Hz}), 128.0(\mathrm{~d}, J=1.2 \mathrm{~Hz}), 127.6(\mathrm{~d}, J=7.3 \mathrm{~Hz}), 127.5,126.2,126.2,126.0,109.6(\mathrm{~m})$, $107.9(\mathrm{~d}, J=7.4 \mathrm{~Hz}), 26.3(\mathrm{~d}, J=30.3 \mathrm{~Hz}) ;{ }^{19} \mathrm{~F}$ NMR $\left(376 \mathrm{MHz}, \mathrm{CDCl}_{3}\right) \delta:-102.8(\mathrm{~m}, 1 \mathrm{~F}),-142.7(\mathrm{dd}$, $J=5.6,14.7 \mathrm{~Hz}, 2 \mathrm{~F}),-155.2(\mathrm{t}, J=20.7,1 \mathrm{~F}),-161.8(\mathrm{~m}, 2 \mathrm{~F})$; HRMS(APCI) $\mathrm{m} / \mathrm{z}:[\mathrm{M}+\mathrm{H}]^{+}$calcd for $\mathrm{C}_{19} \mathrm{H}_{11} \mathrm{~F}_{6} 353.0759$; found 353.0761.

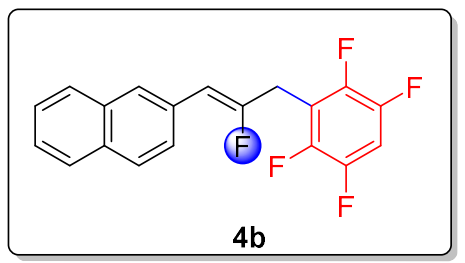

(Z)-2-(2-fluoro-3-(2,3,5,6-tetrafluorophenyl)prop-1-en-1-yl)naphthalene; $\mathrm{Rf}=0.41$ (peterolium ether); Column chromatoghraphy(silica gel; peterolium ether); $69.1 \mathrm{mg}, 69 \%$; white solid; ${ }^{1} \mathrm{H}$ NMR $\left(400 \mathrm{MHz}, \mathrm{CDCl}_{3}\right) \delta: 7.86(\mathrm{~s}, 1 \mathrm{H}), 7.78-7.75(\mathrm{~m}, 3 \mathrm{H}), 7.62(\mathrm{dd}, J=1.6,8.8 \mathrm{~Hz}, 1 \mathrm{H})$, $7.46-7.41(\mathrm{~m}, 2 \mathrm{H}), 7.04-6.99(\mathrm{~m}, 1 \mathrm{H}), 5.73(\mathrm{~d}, J=38.0 \mathrm{~Hz}, 1 \mathrm{H}), 3.80(\mathrm{~d}, J=15.6 \mathrm{~Hz}, 2 \mathrm{H}) ;{ }^{13} \mathrm{C}$ NMR $\left(100 \mathrm{MHz}, \mathrm{CDCl}_{3}\right) \delta: 156.9,154.2,146.8(\mathrm{dm}, J=83.7 \mathrm{~Hz}), 144.3(\mathrm{dm}, J=82.8 \mathrm{~Hz}), 133.3,132.4(\mathrm{~d}$, $J=1.6 \mathrm{~Hz}), 130.3(\mathrm{~d}, J=3.0 \mathrm{~Hz}), 128.0(\mathrm{~d}, J=2.0 \mathrm{~Hz}), 127.5(\mathrm{~d}, J=7.3 \mathrm{~Hz}), 127.5,126.3(\mathrm{~d}, J=7.6 \mathrm{~Hz})$, $126.1,126.0,115.5(\mathrm{~m}), 107.8(\mathrm{~d}, J=7.4 \mathrm{~Hz}), 105.1(\mathrm{~m}), 26.3(\mathrm{~d}, J=30.3 \mathrm{~Hz}) ;{ }^{19} \mathrm{~F}$ NMR $(376 \mathrm{MHz}$, $\left.\mathrm{CDCl}_{3}\right) \delta:-102.2(\mathrm{~m}, 1 \mathrm{~F}),-138.9(\mathrm{~m}, 2 \mathrm{~F}),-143.2(\mathrm{~m}, 2 \mathrm{~F})$; HRMS(APCI) $\mathrm{m} / \mathrm{z}:[\mathrm{M}+\mathrm{H}]^{+}$calcd for $\mathrm{C}_{19} \mathrm{H}_{12} \mathrm{~F}_{5} 335.0854$; found 335.0856 .

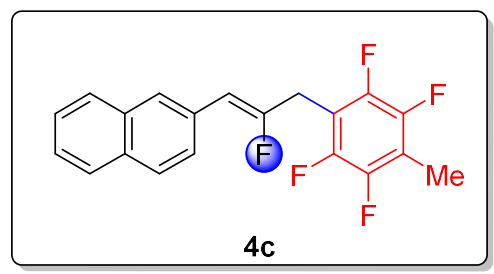

(Z)-2-(2-fluoro-3-(2,3,5,6-tetrafluoro-4-methylphenyl)prop-1-en-1-yl)naphthalene; 
$\mathrm{Rf}=0.39$ (peterolium ether); Column chromatoghraphy (silica gel; peterolium ether); $70.0 \mathrm{mg}, 67 \%$; white solid; ${ }^{1} \mathrm{H}$ NMR $\left(400 \mathrm{MHz}, \mathrm{CDCl}_{3}\right) \delta: 7.85(\mathrm{~s}, 1 \mathrm{H}), 7.76(\mathrm{dd}, J=3.6,4.8 \mathrm{~Hz}, 3 \mathrm{H}), 7.60(\mathrm{dd}$, $J=1.6,8.8 \mathrm{~Hz}, 1 \mathrm{H}), 7.45-7.40(\mathrm{~m}, 2 \mathrm{H}), 5.70(\mathrm{~d}, J=38.4 \mathrm{~Hz}, 1 \mathrm{H}), 3.77(\mathrm{~d}, J=15.2 \mathrm{~Hz}, 2 \mathrm{H}), 2.26(\mathrm{~s}, 3 \mathrm{H})$; ${ }^{13} \mathrm{C}$ NMR $\left(100 \mathrm{MHz}, \mathrm{CDCl}_{3}\right) \delta: 157.4,154.7,146.1(\mathrm{~m}), 143.7(\mathrm{~m}), 133.3,132.4(\mathrm{~d}, J=1.7 \mathrm{~Hz})$, $130.4(\mathrm{~d}, J=3.0 \mathrm{~Hz}), 127.9(\mathrm{~d}, J=4.8 \mathrm{~Hz}), 127.5,127.4,126.3(\mathrm{~d}, J=7.7 \mathrm{~Hz}), 126.1,125.9,115.3(\mathrm{t}$, $J=19.0 \mathrm{~Hz}), 111.6(\mathrm{~m}), 107.5(\mathrm{~d}, J=7.6 \mathrm{~Hz}), 26.5(\mathrm{~d}, J=30.6 \mathrm{~Hz}), 7.5 ;{ }^{19} \mathrm{~F}$ NMR $\left(376 \mathrm{MHz}, \mathrm{CDCl}_{3}\right) \delta$ : $-101.9(\mathrm{~m}, 1 \mathrm{~F}),-143.8(\mathrm{~m}, 2 \mathrm{~F}),-145.0(\mathrm{~m}, 2 \mathrm{~F})$; HRMS(APCI) $\mathrm{m} / \mathrm{z}:[\mathrm{M}+\mathrm{H}]^{+}$calcd for $\mathrm{C}_{20} \mathrm{H}_{14} \mathrm{~F}_{5}$ 349.1010; found 349.1015.

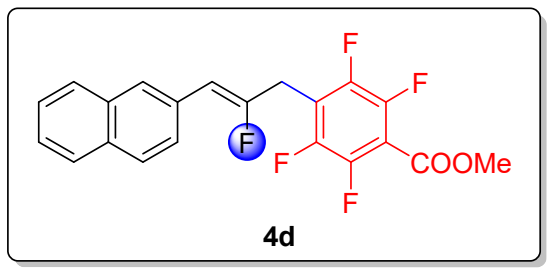

\section{Methyl(Z)-2,3,5,6-tetrafluoro-4-(2-fluoro-3-(naphthalen-2-yl)allyl)benzoate;}

$\mathrm{Rf}=0.33$ (peterolium ether/ethyl acetate, 30:1); Column chromatoghraphy(silica gel; peterolium ether/ethyl acetate, 70:1); 77.6mg, 66\%; Z/E=9:1; yellow solid; ${ }^{1} \mathrm{H}$ NMR $\left(400 \mathrm{MHz}, \mathrm{CDCl}_{3}\right) \delta$ : $7.85(\mathrm{~s}, 1 \mathrm{H}), 7.78-7.75(\mathrm{~m}, 3 \mathrm{H}), 7.59(\mathrm{dd}, J=1.6,8.4 \mathrm{~Hz}, 1 \mathrm{H}), 7.45-7.42(\mathrm{~m}, 2 \mathrm{H}), 5.75(\mathrm{~d}, J=38.0 \mathrm{~Hz}$, $1 \mathrm{H}), 3.97(\mathrm{~s}, 3 \mathrm{H}), 3.81(\mathrm{~d}, J=16.0 \mathrm{~Hz}, 2 \mathrm{H}) ;{ }^{13} \mathrm{C} \mathrm{NMR}\left(100 \mathrm{MHz}, \mathrm{CDCl}_{3}\right) \delta: 160.0,156.1,153.4$, $146.2(\mathrm{dm}, J=55.8 \mathrm{~Hz}), 143.5(\mathrm{dm}, J=63.8 \mathrm{~Hz}), 133.2,132.4(\mathrm{~d}, J=1.7 \mathrm{~Hz}), 130.1(\mathrm{~d}, J=3.1 \mathrm{~Hz}), 128.0$, $127.6(\mathrm{~d}, J=7.2 \mathrm{~Hz}), 127.4,126.2,126.1,126.0,118.1(\mathrm{~m}), 111.7(\mathrm{~m}), 108.3(\mathrm{~d}, J=7.3 \mathrm{~Hz}), 53.2$, $26.9(\mathrm{~d}, J=30.6 \mathrm{~Hz}) ;{ }^{19} \mathrm{~F}$ NMR (376 MHz, $\left.\mathrm{CDCl}_{3}\right) \delta:-102.5(\mathrm{~m}, 1 \mathrm{~F}),-139.4(\mathrm{~m}, 2 \mathrm{~F}),-141.7(\mathrm{~m}, 2 \mathrm{~F})$; HRMS(APCI) m/z: [M+H] calcd for $\mathrm{C}_{21} \mathrm{H}_{14} \mathrm{~F}_{5} \mathrm{O}_{2}$ 393.0908; found 393.0903 .

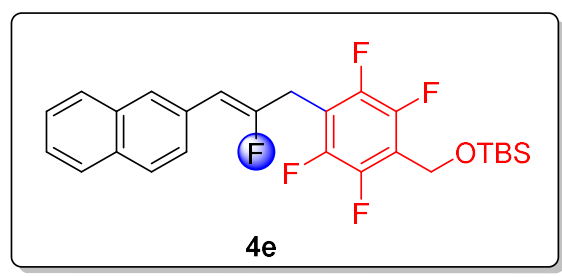

(Z)-tert-butyldimethyl((2,3,5,6-tetrafluoro-4-(2-fluoro-3-(naphthalen-2-yl)allyl)benzyl)oxy)sil ane; $\mathrm{Rf}=0.30$ (peterolium ether/ethyl acetate, 100:1); Column chromatoghraphy(silica gel; peterolium ether/ethyl acetate, 200:1); $114.7 \mathrm{mg}, 80 \%$; white solid; ${ }^{1} \mathrm{H} \mathrm{NMR}\left(400 \mathrm{MHz}, \mathrm{CDCl}_{3}\right) \delta$ : $7.73(\mathrm{~s}, 1 \mathrm{H}), 7.65-7.62(\mathrm{~m}, 3 \mathrm{H}), 7.49(\mathrm{~d}, J=8.0 \mathrm{~Hz}, 1 \mathrm{H}), 7.32-7.29(\mathrm{~m}, 2 \mathrm{H}), 5.60(\mathrm{~d}, J=38.4 \mathrm{~Hz}, 1 \mathrm{H})$, $4.65(\mathrm{~s}, 2 \mathrm{H}), 3.65(\mathrm{~d}, J=15.6 \mathrm{~Hz}, 2 \mathrm{H}), 0.79(\mathrm{~s}, 9 \mathrm{H}), 0.01(\mathrm{~s}, 6 \mathrm{H}) ;{ }^{13} \mathrm{C} \mathrm{NMR}\left(100 \mathrm{MHz}, \mathrm{CDCl}_{3}\right) \delta$ : $157.0,154.3,146.1(\mathrm{~m}), 143.6(\mathrm{~m}), 133.3,132.4(\mathrm{~d}, J=1.6 \mathrm{~Hz}), 130.3(\mathrm{~d}, J=3.0 \mathrm{~Hz}), 127.9(\mathrm{~d}$, $J=3.4 \mathrm{~Hz}), 127.5(\mathrm{~d}, J=7.4 \mathrm{~Hz}), 127.4,126.3(\mathrm{~d}, J=7.7 \mathrm{~Hz}), 126.1,125.9,118.1(\mathrm{t}, J=17.5 \mathrm{~Hz})$, $114.2(\mathrm{~m}), 107.7(\mathrm{~d}, J=7.4 \mathrm{~Hz}), 53.2,26.7(\mathrm{~d}, J=30.6 \mathrm{~Hz}), 25.7,18.3,-5.5 ;{ }^{19} \mathrm{~F}$ NMR $(376 \mathrm{MHz}$, $\left.\mathrm{CDCl}_{3}\right) \delta:-102.0(\mathrm{~m}, 1 \mathrm{~F}),-143.8(\mathrm{~m}, 2 \mathrm{~F}),-144.4(\mathrm{~m}, 2 \mathrm{~F})$; HRMS(ESI) $\mathrm{m} / \mathrm{z}:[\mathrm{M}+\mathrm{Na}]^{+}$calcd for $\mathrm{C}_{26} \mathrm{H}_{27} \mathrm{~F}_{5} \mathrm{OSiNa}$ 501.1644; found 501.1641.

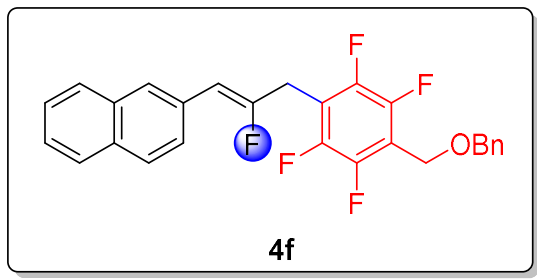


(Z)-2-(3-(4-((benzyloxy)methyl)-2,3,5,6-tetrafluorophenyl)-2-fluoroprop-1-en-1-yl)naphthale ne; $\mathrm{Rf}=0.32$ (peterolium ether/ethyl acetate, 100:1); Column chromatoghraphy(silica gel; peterolium ether/ethyl acetate, 200:1); 106.2mg, 78\%; yellow solid; ${ }^{1} \mathrm{H}$ NMR $\left(400 \mathrm{MHz}, \mathrm{CDCl}_{3}\right) \delta$ : $7.84(\mathrm{~s}, 1 \mathrm{H}), \quad 7.76-7.73(\mathrm{~m}, 3 \mathrm{H}), 7.61-7.58(\mathrm{~m}, 1 \mathrm{H}), 7.42-7.40(\mathrm{~m}, 2 \mathrm{H}), 7.34-7.31(\mathrm{~m}, 4 \mathrm{H})$, $7.29-7.27(\mathrm{~m}, 1 \mathrm{H}), 5.71(\mathrm{~d}, J=38.0 \mathrm{~Hz}, 1 \mathrm{H}), 4.62(\mathrm{~s}, 2 \mathrm{H}), 4.57(\mathrm{~s}, 2 \mathrm{H}), 3.76(\mathrm{~d}, J=15.6 \mathrm{~Hz}, 2 \mathrm{H}) ;{ }^{13} \mathrm{C}$ NMR $\left(100 \mathrm{MHz}, \mathrm{CDCl}_{3}\right) \delta: 156.8,154.2,146.2(\mathrm{~m}), 143.8(\mathrm{~m}), 137.3,133.2,132.4(\mathrm{~d}, J=1.5 \mathrm{~Hz})$, $130.3(\mathrm{~d}, J=3.0 \mathrm{~Hz}), 128.4,127.9,127.9,127.8,127.7,127.5(\mathrm{~d}, J=7.2 \mathrm{~Hz}), 127.4,126.2(\mathrm{~d}, J=7.6 \mathrm{~Hz})$, $126.1,125.9,115.3(\mathrm{dm}, J=52.5 \mathrm{~Hz}), 107.8(\mathrm{~d}, J=7.4 \mathrm{~Hz}), 72.9,59.1,26.7(\mathrm{~d}, J=30.5 \mathrm{~Hz}) ;{ }^{19} \mathrm{~F}$ NMR $\left(376 \mathrm{MHz}, \mathrm{CDCl}_{3}\right) \delta$ : $-102.0(\mathrm{~m}, 1 \mathrm{~F}),-143.4(\mathrm{~s}, 4 \mathrm{~F})$; HRMS(ESI) $\mathrm{m} / \mathrm{z}:[\mathrm{M}+\mathrm{Na}]^{+}$calcd for $\mathrm{C}_{27} \mathrm{H}_{19} \mathrm{~F}_{5} \mathrm{ONa}$ 477.1248; found 477.1246.

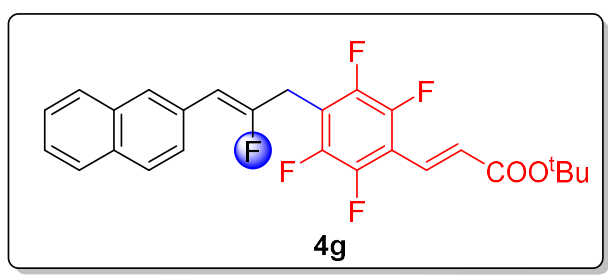

tert-butyl (E)-3-(2,3,5,6-tetrafluoro-4-((Z)-2-fluoro-3-(naphthalen-2-yl)allyl)phenyl)acrylate: $\mathrm{Rf}=0.31$ (peterolium ether/ethyl acetate, 30:1); Column chromatoghraphy(silica gel; peterolium ether/ethyl acetate, 60:1); 114.6mg, 83\%; yellow oil; ${ }^{1} \mathrm{H} \mathrm{NMR}\left(400 \mathrm{MHz}, \mathrm{CDCl}_{3}\right) \delta: 7.78(\mathrm{~s}, 1 \mathrm{H})$, $7.70-7.67(\mathrm{~m}, 3 \mathrm{H}), 7.55-7.51(\mathrm{~m}, 2 \mathrm{H}), 7.37-7.35(\mathrm{~m}, 2 \mathrm{H}), 6.62(\mathrm{~d}, J=16.8 \mathrm{~Hz}, 1 \mathrm{H}), 5.68(\mathrm{~d}, J=38.0 \mathrm{~Hz}$, $1 \mathrm{H}), 3.73(\mathrm{~d}, J=16.0 \mathrm{~Hz}, 2 \mathrm{H}), 1.46(\mathrm{~s}, 9 \mathrm{H}) ;{ }^{13} \mathrm{C} \mathrm{NMR}\left(100 \mathrm{MHz}, \mathrm{CDCl}_{3}\right) \delta: 165.3,156.5,153.8$, $145.1(\mathrm{dm}, J=252.4 \mathrm{~Hz}), 133.3,132.4(\mathrm{~d}, J=1.6 \mathrm{~Hz}), 130.2(\mathrm{~d}, J=3.1 \mathrm{~Hz}), 128.2(\mathrm{t}, J=8.4 \mathrm{~Hz}), 128.0$, $127.6(\mathrm{~d}, J=7.2 \mathrm{~Hz}), 127.5,126.2(\mathrm{~d}, J=7.6 \mathrm{~Hz}), 126.1,126.0,115.6(\mathrm{~m}), 113.7(\mathrm{t}, J=13.1 \mathrm{~Hz}), 108.1(\mathrm{~d}$, $J=7.3 \mathrm{~Hz}), 81.3,28.0,26.9(\mathrm{~d}, J=30.4 \mathrm{~Hz}) ;{ }^{19} \mathrm{~F} \mathrm{NMR}\left(376 \mathrm{MHz}, \mathrm{CDCl}_{3}\right) \delta:-102.3(\mathrm{t}, J=5.2 \mathrm{~Hz}, 1 \mathrm{~F})$, $-140.5(\mathrm{~m}, 2 \mathrm{~F}),-143.4(\mathrm{~m}, 2 \mathrm{~F})$; HRMS(ESI) $\mathrm{m} / \mathrm{z}$ : $\left[\mathrm{M}+\mathrm{NH}_{4}\right]^{+}$calcd for $\mathrm{C}_{26} \mathrm{H}_{25} \mathrm{~F}_{5} \mathrm{NO}_{2} 478.1800$; found 478.1800 .

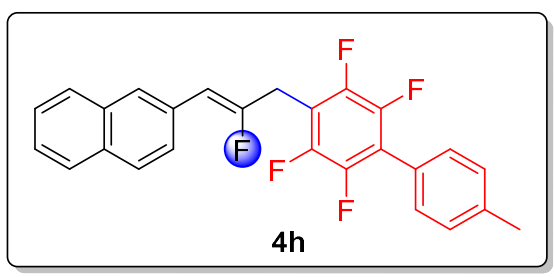

(Z)-2-(2-fluoro-3-(2,3,5,6-tetrafluoro-4'-methyl-[1,1'-biphenyl]-4-yl)prop-1-en-1-yl)naphthale ne: $\mathrm{Rf}=0.37$ (peterolium ether); Column chromatoghraphy(silica gel; peterolium ether); $45.6 \mathrm{mg}$, 36\%; Z/E=19:1; white solid; ${ }^{1} \mathrm{H}$ NMR $\left(400 \mathrm{MHz}, \mathrm{CDCl}_{3}\right) \delta$ : 7.89(s, $\left.1 \mathrm{H}\right), 7.79-7.76(\mathrm{~m}, 3 \mathrm{H})$, $7.63(\mathrm{dd}, J=1.6,8.4 \mathrm{~Hz}, 1 \mathrm{H}), 7.445-7.42(\mathrm{~m}, 2 \mathrm{H}), 7.36(\mathrm{~d}, J=8.0 \mathrm{~Hz}, 2 \mathrm{H}), 7.29(\mathrm{~d}, J=8.0 \mathrm{~Hz}, 2 \mathrm{H})$, $5.78(\mathrm{~d}, J=38.0 \mathrm{~Hz}, 1 \mathrm{H}), 3.84(\mathrm{~d}, J=15.6 \mathrm{~Hz}, 2 \mathrm{H}), 2.40(\mathrm{~s}, 3 \mathrm{H}) ;{ }^{13} \mathrm{C} \mathrm{NMR}\left(100 \mathrm{MHz}, \mathrm{CDCl}_{3}\right) \delta: 157.1$, $154.5,145.4(\mathrm{dm}, J=251.4 \mathrm{~Hz}), 143.7(\mathrm{dm}, J=235.6 \mathrm{~Hz}), 139.2,133.3,132.4(\mathrm{~d}, J=1.6 \mathrm{~Hz}), 130.4(\mathrm{~d}$, $J=3.1 \mathrm{~Hz}), 129.9,129.3,128.0(\mathrm{t}, J=3.1 \mathrm{~Hz}), 127.6(\mathrm{~d}, J=7.3 \mathrm{~Hz}), 127.5,126.3(\mathrm{~d}, J=7.6 \mathrm{~Hz}), 126.1$, 126.0, 124.3, 119.9(m), 113.2(m), 107.8(d, J=7.4Hz), 26.8(d, J=30.6Hz), 21.3; ${ }^{19} \mathrm{~F}$ NMR $\left(376 \mathrm{MHz}, \mathrm{CDCl}_{3}\right) \delta:-102.02(\mathrm{~m}, 1 \mathrm{~F}),-143.8(\mathrm{~m}, 2 \mathrm{~F}),-144.1(\mathrm{~m}, 2 \mathrm{~F}) ; \operatorname{HRMS}(\mathrm{ESI}) \mathrm{m} / \mathrm{z}:[\mathrm{M}+\mathrm{H}]^{+}$ calcd for $\mathrm{C}_{26} \mathrm{H}_{18} \mathrm{~F}_{5}$ 425.1323; found 425.1339 . 


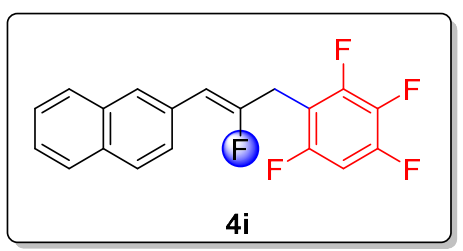

(Z)-2-(2-fluoro-3-(2,3,4,6-tetrafluorophenyl)prop-1-en-1-yl)naphthalene; $\mathrm{Rf}=0.39$ (peterolium ether); Column chromatoghraphy(silica gel; peterolium ether); $70.1 \mathrm{mg}, 70 \%$; white solid; ${ }^{1} \mathrm{H}$ NMR $\left(400 \mathrm{MHz}, \mathrm{CDCl}_{3}\right) \delta: 7.84(\mathrm{~s}, 1 \mathrm{H}), 7.77-7.74(\mathrm{~m}, 3 \mathrm{H}), 7.59(\mathrm{dd}, J=1.6,8.4 \mathrm{~Hz}, 1 \mathrm{H})$, $7.43-7.41(\mathrm{~m}, 2 \mathrm{H}), 6.82-6.75(\mathrm{~m}, 1 \mathrm{H}), 5.68(\mathrm{~d}, J=38.4 \mathrm{~Hz}, 1 \mathrm{H}), 3.70(\mathrm{~d}, J=15.2 \mathrm{~Hz}, 2 \mathrm{H}) ;{ }^{13} \mathrm{C}$ NMR $\left(100 \mathrm{MHz}, \mathrm{CDCl}_{3}\right) \delta: 157.4,155.6(\mathrm{dm}, J=245.5 \mathrm{~Hz}), 154.7,150.2(\mathrm{dm}, J=249.3 \mathrm{~Hz}), 137.2(\mathrm{dm}$, $J=247.4 \mathrm{~Hz}), 133.3,132.4,130.4(\mathrm{~d}, J=3.0 \mathrm{~Hz}), 127.9,127.9,127.5,127.5,126.3(\mathrm{~d}, J=7.6 \mathrm{~Hz})$, $126.1,125.9,109.6(\mathrm{~d}, J=18.1 \mathrm{~Hz}), 107.4(\mathrm{~d}, J=7.5 \mathrm{~Hz}), 100.8(\mathrm{~m}), 26.3(\mathrm{~d}, J=30.5 \mathrm{~Hz}) ;{ }^{19} \mathrm{~F}$ NMR $\left(376 \mathrm{MHz}, \mathrm{CDCl}_{3}\right) \delta:-102.1(\mathrm{t}, J=4.9 \mathrm{~Hz}, 1 \mathrm{~F}),-118.1(\mathrm{dd}, J=4.9,11.3 \mathrm{~Hz}, 1 \mathrm{~F}),-133.1(\mathrm{~m}, 1 \mathrm{~F})$, -133.2(m, 1F), -164.6(m, 1F); HRMS(ESI) m/z: $[\mathrm{M}+\mathrm{H}]^{+}$calcd for $\mathrm{C}_{19} \mathrm{H}_{12} \mathrm{~F}_{5}$ 335.0854; found 335.0856 .

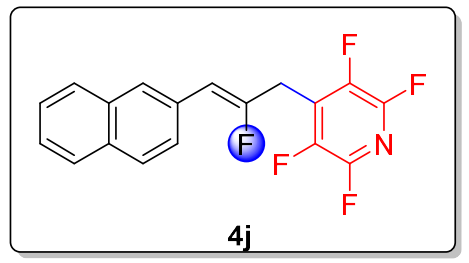

(Z)-2,3,5,6-tetrafluoro-4-(2-fluoro-3-(naphthalen-2-yl)allyl)pyridine; $\quad \mathrm{Rf}=0.32$ (peterolium ether/ethyl acetate, 100:1); Column chromatoghraphy(silica gel; peterolium ether/ethyl acetate, 200:1); 41.2mg, 41\%; Z/E=15:1; white solid; ${ }^{1} \mathrm{H}$ NMR (400MHz, $\left.\mathrm{CDCl}_{3}\right) \delta: 7.87-7.77(\mathrm{~m}, 4 \mathrm{H})$, $7.60(\mathrm{~d}, J=1.6,8.4 \mathrm{~Hz}, 1 \mathrm{H}), 7.47-7.44(\mathrm{~m}, 2 \mathrm{H}), 5.83(\mathrm{~d}, J=37.6 \mathrm{~Hz}, 1 \mathrm{H}), 3.88(\mathrm{~d}, J=16.8 \mathrm{~Hz}, 2 \mathrm{H}) ;{ }^{13} \mathrm{C}$ NMR (100MHz, $\left.\mathrm{CDCl}_{3}\right) \delta: 154.8,152.2,143.3(\mathrm{dm}, J=238.2 \mathrm{~Hz}), 140.7(\mathrm{dm}, J=236.2 \mathrm{~Hz}), 133.2$, $132.6(\mathrm{~d}, J=1.5 \mathrm{~Hz}), 129.8(\mathrm{~d}, J=3.2 \mathrm{~Hz}), 128.1,128.0,127.8(\mathrm{~d}, J=7.3 \mathrm{~Hz}), 127.6(\mathrm{~d}, J=6.9 \mathrm{~Hz}), 127.5$, $126.2(\mathrm{~d}, J=2.6 \mathrm{~Hz}), 126.1(\mathrm{~d}, J=7.6 \mathrm{~Hz}), 109.1(\mathrm{~d}, J=7.1 \mathrm{~Hz}), 27.6(\mathrm{~d}, J=30.3 \mathrm{~Hz}) ;{ }^{19} \mathrm{~F}$ NMR $(376 \mathrm{MHz}$, $\left.\mathrm{CDCl}_{3}\right) \delta$ : $-90.4(\mathrm{~m}, 2 \mathrm{~F}),-103.0(\mathrm{~m}, 1 \mathrm{~F}),-143.8(\mathrm{~m}, 2 \mathrm{~F})$; HRMS(ESI) $\mathrm{m} / \mathrm{z}:[\mathrm{M}+\mathrm{H}]^{+}$calcd for $\mathrm{C}_{18} \mathrm{H}_{11} \mathrm{~F}_{5} \mathrm{~N} 336.0806$; found 336.0806.

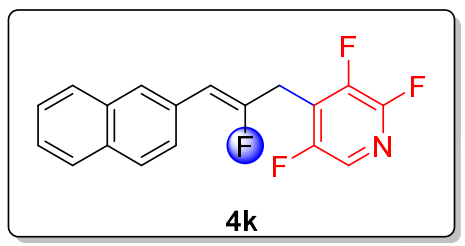

(Z)-2,3,5-trifluoro-4-(2-fluoro-3-(naphthalen-2-yl)allyl)pyridine: $\mathrm{Rf}=0.31$ (peterolium ether/ ethyl acetate, 100:1); Column chromatoghraphy(silica gel; peterolium ether/ethyl acetate, 200:1); 60.1mg, 63\%; Z/E=11:1; white solid; ${ }^{1} \mathrm{H}$ NMR $\left(400 \mathrm{MHz}, \mathrm{CDCl}_{3}\right) \delta: 7.79(\mathrm{~d}, J=13.2 \mathrm{~Hz}, 2 \mathrm{H})$, $7.70-7.68(\mathrm{~m}, 3 \mathrm{H}), 7.52(\mathrm{~d}, J=8.8 \mathrm{~Hz}, 1 \mathrm{H}), 7.36(\mathrm{dd}, J=4.0,5.6 \mathrm{~Hz}, 2 \mathrm{H}), 5.70(\mathrm{~d}, J=38.0 \mathrm{~Hz}, 1 \mathrm{H})$, $3.75(\mathrm{~d}, J=16.0 \mathrm{~Hz}, 2 \mathrm{H}) ;{ }^{13} \mathrm{C}$ NMR $\left(100 \mathrm{MHz}, \mathrm{CDCl}_{3}\right) \delta: 155.5(\mathrm{dd}, J=4.0,254.9 \mathrm{~Hz}), 154.2(\mathrm{~d}$, $J=266.1 \mathrm{~Hz}), 148.2(\mathrm{dm}, J=237.2 \mathrm{~Hz}), 143.9(\mathrm{dm}, J=259.7 \mathrm{~Hz}), 133.2,132.5(\mathrm{~d}, J=1.6 \mathrm{~Hz}), 130.0(\mathrm{~d}$, $J=3.1 \mathrm{~Hz}), 128.2(\mathrm{~m}), 128.0,128.0,127.7(\mathrm{~d}, J=7.3 \mathrm{~Hz}), 127.5,126.2,126.2,126.1,125.0(\mathrm{~m})$, $108.6(\mathrm{~d}, J=7.3 \mathrm{~Hz}), 27.0(\mathrm{~d}, J=30.5 \mathrm{~Hz}) ;{ }^{19} \mathrm{~F}$ NMR $\left(376 \mathrm{MHz}, \mathrm{CDCl}_{3}\right) \delta:-88.8(\mathrm{q}, J=25.6 \mathrm{~Hz}, 1 \mathrm{~F})$, $-102.5(\mathrm{~m}, 1 \mathrm{~F}),-131.8(\mathrm{~m}, 1 \mathrm{~F}),-139.3(\mathrm{~m}, 1 \mathrm{~F})$; HRMS(ESI) $\mathrm{m} / \mathrm{z}$ : $[\mathrm{M}+\mathrm{H}]^{+}$calcd for $\mathrm{C}_{18} \mathrm{H}_{12} \mathrm{~F}_{4} \mathrm{~N}$ 318.0900; found 318.0895. 


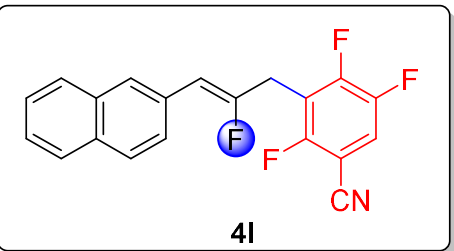

(Z)-2,4,5-trifluoro-3-(2-fluoro-3-(naphthalen-2-yl)allyl)benzonitrile; $\quad \mathrm{Rf}=0.28$ (peterolium ether/ethyl acetate, 40:1); Column chromatoghraphy (silica gel; peterolium ether/ethyl acetate, 80:1); 45.0mg, 44\%; yellow solid; ${ }^{1} \mathrm{H}$ NMR (400MHz, $\left.\mathrm{CDCl}_{3}\right) \delta: 7.86(\mathrm{~s}, 1 \mathrm{H}), 7.77(\mathrm{~d}, J=7.6 \mathrm{~Hz}$, $3 \mathrm{H}), 7.61-7.59(\mathrm{~m}, 1 \mathrm{H}), 7.46-7.36(\mathrm{~m}, 3 \mathrm{H}), 5.78(\mathrm{~d}, J=38.0 \mathrm{~Hz}, 1 \mathrm{H}), 3.80(\mathrm{~d}, J=16.4 \mathrm{~Hz}, 2 \mathrm{H}) ;{ }^{13} \mathrm{C}$ NMR $\left(100 \mathrm{MHz}, \mathrm{CDCl}_{3}\right) \delta: 158.2(\mathrm{dm}, J=256.9 \mathrm{~Hz}), 155.9,153.2,152.7(\mathrm{dm}, J=259.7 \mathrm{~Hz})$, $146.9(\mathrm{dm}, J=245.9 \mathrm{~Hz}), 133.2,132.5(\mathrm{~d}, J=1.6 \mathrm{~Hz}), 129.9(\mathrm{~d}, J=3.2 \mathrm{~Hz}), 128.0,127.9,127.7(\mathrm{~d}$, $J=7.3 \mathrm{~Hz}), 127.5,126.2,126.1,119.3(\mathrm{~d}, J=21.7 \mathrm{~Hz}), 116.3(\mathrm{~m}), 112.1,108.5(\mathrm{~d}, J=7.3 \mathrm{~Hz}), 97.3(\mathrm{~m})$, 26.6(d, $J=28.4 \mathrm{~Hz}) ;{ }^{19} \mathrm{~F}$ NMR $\left(376 \mathrm{MHz}, \mathrm{CDCl}_{3}\right) \delta:-103.0(\mathrm{~m}, 1 \mathrm{~F}),-110.9(\mathrm{~m}, 1 \mathrm{~F}),-125.2(\mathrm{dd}, J=1.1$, $21.1 \mathrm{~Hz}, 1 \mathrm{~F}),-137.5(\mathrm{dd}, J=14.7,21.4 \mathrm{~Hz}, 1 \mathrm{~F}) ; \mathrm{HRMS}(\mathrm{APCI}) \mathrm{m} / \mathrm{z}:[\mathrm{M}+\mathrm{H}]^{+}$calcd for $\mathrm{C}_{20} \mathrm{H}_{12} \mathrm{~F}_{4} \mathrm{~N}$ 342.0900; found 342.0900.

\section{References}

1. (a) J. Xu, E.-A. Ahmed, B. Xiao, Q.-Q. Lu, Y.-L. Wang, C.-G. Yu and Y. Fu, Pd-Catalyzed Regioselective Activation of gem-Difluorinated Cyclopropanes: A Highly Efficient Approach to 2-Fluorinated Allylic Scaffolds, Angew. Chem., Int. Ed, 2015, 54, 8231-8235;

2. (a) L. Li, F. Wang, C. Ni, J. Hu, Synthesis of gem-Difluorocyclopropa(e)nes and O-, S-, N-, and P-Difluoromethylated Compounds with $\mathrm{TMSCF}_{2} \mathrm{Br}$, Angew. Chem. Int. Ed, 2013, 52, 12390-12394; (b) P. Rulliere, P. Cyr, A. B. Charette, Difluorocarbene Addition to Alkenes and Alkynes in Continuous Flow, Org. Lett. 2016, 18, 1988-1991; (c) J. Ni, B. Nishonov, A. Pardaev, A. Zhang, Palladium-Catalyzed Ring-Opening Coupling of gem-Difluorocyclopropanes for the Construction of 2-Fluoroallylic Sulfones, J. Org. Chem. 2019, 84, 13646-13654; (d) H. Liu, Y. Li, D.-X. Wang, M.-M. Sun, C. Feng, Visible-Light-Promoted Regioselective 1,3-Fluoroallylation of gem-Difluorocyclopropanes, Org. Lett. 2020, 22, 8681-8686. (e) Z. Fu, J. Zhu, S. Guo A. Lin, Palladium-catalyzed Allylic Alkylation Dearomatization of $\beta$-Naphthols and Indoles with gem-Difluorinated Cyclopropanes, Chem. Commun. 2021, 57, 1262-1265.

3. X. Shao and S. J. Malcolmson, Catalytic Enantio- and Diastereoselective Cyclopropanation of 2-Azadienes for the Synthesis of Aminocyclopropanes Bearing Quaternary Carbon Stereogenic Centers, Org. Lett. 2019, 21, 7380-7385.

4. A. K. Chakraborti and S. V. Chankeshwara, Counterattack Mode Differential Acetylative Deprotection of Phenylmethyl Ethers:Applications to Solid Phase Organic Reactions, J. Org. Chem. 2009, 74, 1367-1370.

\section{The crystal structure of product $3 \mathrm{e}$}
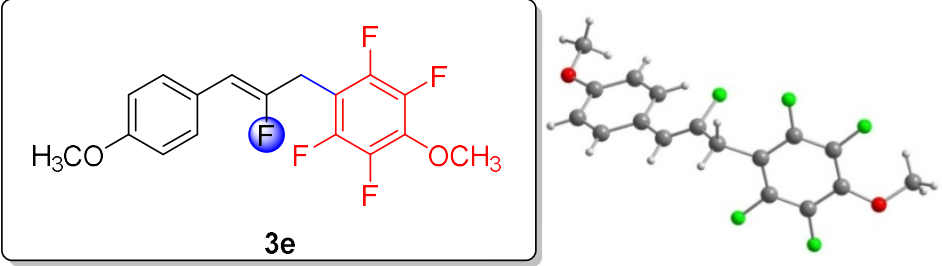

(Deposition Number 2071205) 


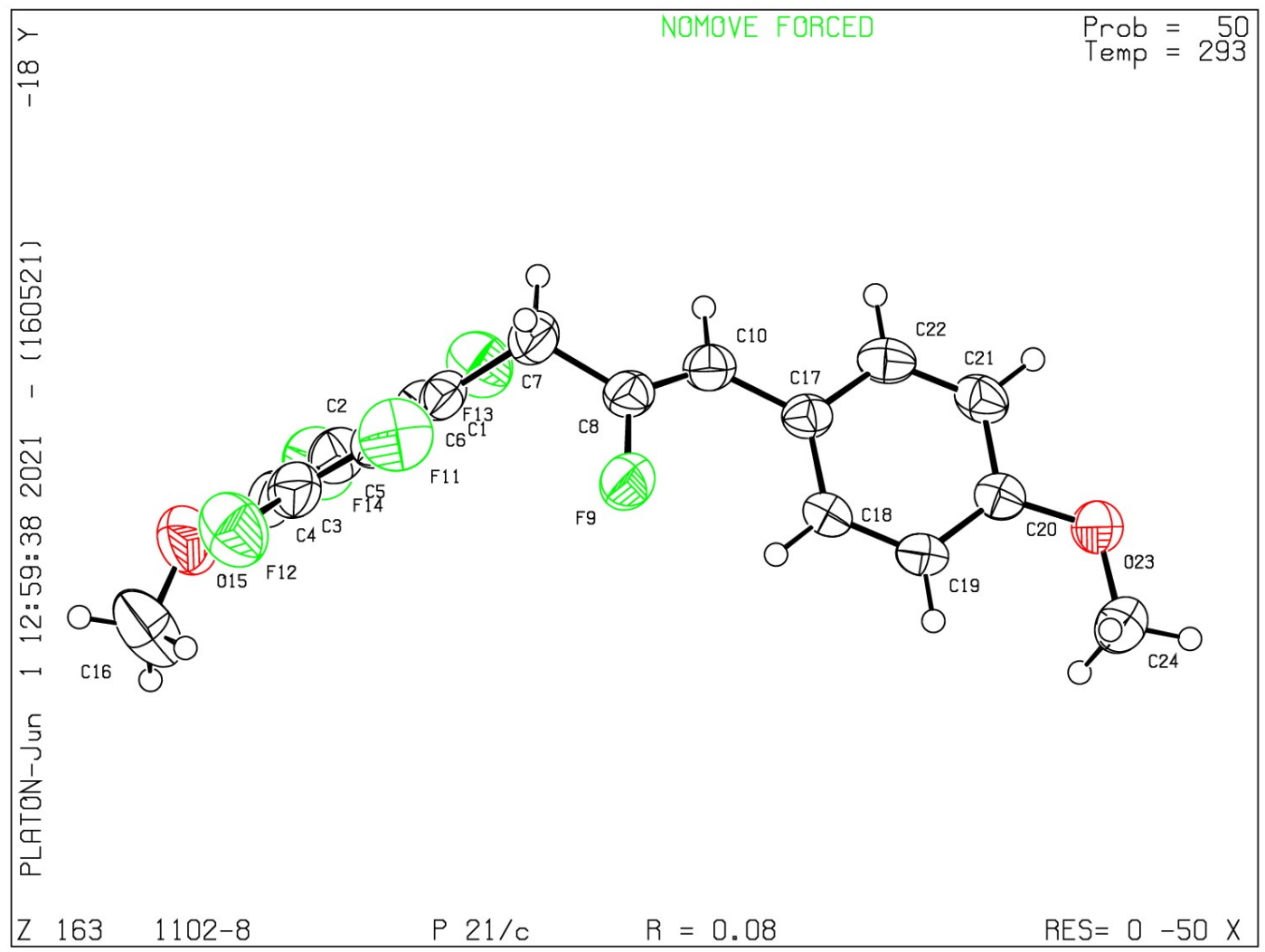

Thermal ellipsoids are drawn at 50\% probability level.

$\begin{array}{lllll}\text { Bond precision: } & \mathrm{C}-\mathrm{C}=0.0049 \mathrm{~A} & & \text { Wavelength }=0.71073 \\ \text { Cell: } & \mathrm{a}=28.345(3) & \mathrm{b}=5.6893(5) & \mathrm{c}=9.3090(11) & \\ & \text { alpha }=90 & \text { beta }=93.244(9) & \text { gamma }=90 & \end{array}$

Temperature: $293 \mathrm{~K}$

$\begin{array}{lll} & \text { Calculated } & \text { Reported } \\ \text { Volume } & 1498.8(3) & 1498.8(3) \\ \text { Space group } & \text { P 21/c } & \text { P 21/c } \\ \text { Hall group } & -P \text { 2ybc } & -P \text { 2ybc } \\ \text { Moiety formula } & \text { C17 H13 F5 O2 } & ? \\ \text { Sum formula } & \text { C17 H13 F5 O2 } & \text { C17 H13 F5 O2 } \\ \text { Mr } & 344.27 & 344.27 \\ \text { Dx,g cm-3 } & 1.526 & 1.526 \\ \text { Z } & 4 & 4 \\ \text { Mu (mm-1) } & 0.140 & 0.140 \\ \text { F000 } & 704.0 & 704.0 \\ \text { F000' } & 704.58 & \\ \text { h,k,lmax } & 38,7,12 & 38,7,12 \\ \text { Nref } & 3990 & 3529 \\ \text { Tmin,Tmax } & & \end{array}$


Tmin'

Correction method $=$ Not given

Data completeness $=0.884$

Theta $(\max )=28.999$

$\mathrm{R}($ reflections $)=0.0763(2054)$

$w R 2$ (reflections) $=0.1838(3529)$

$\mathrm{S}=1.037$

Npar $=219$

Sample preparation: A solution of compound $3 \mathbf{e}(100 \mathrm{mg})$ in ethyl acetate was placed in a vial (5 $\mathrm{mL}$ ). Petroleum ether was added to the vial with a dropper until a small amount of solid precipitation, then, added the ethyl acetate until the solution clarification. The single crystal $\mathbf{3 e}$ was obtained by slowly evaporating mixed solvent at room temperature under the air conditions.

Crystal measurement: X-ray crystal structures were determined at $293 \mathrm{~K}$. The crystal was measured on a SuperNova, Dual, $\mathrm{Cu}$ at zero, Eos diffractometer. 
8. ${ }^{1} \mathrm{H},{ }^{13} \mathrm{C}$ and ${ }^{19} \mathrm{~F}$ NMR spectra for compound $1 \mathrm{~h}, 1 \mathrm{p}, 1 \mathrm{r}, 2 \mathrm{e}, 2 \mathrm{f}, 3$ and 4
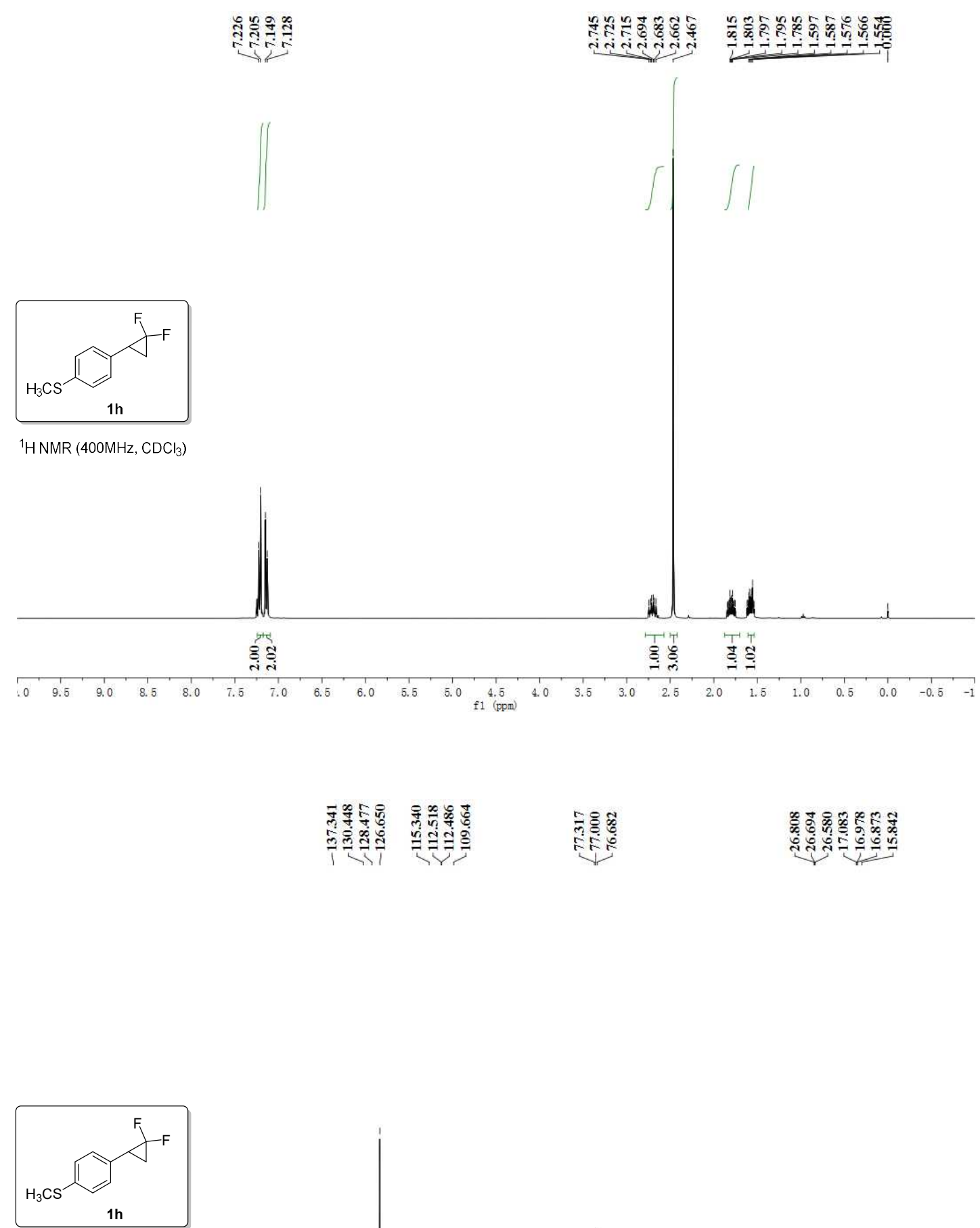

${ }^{13} \mathrm{C} \mathrm{NMR}\left(100 \mathrm{MHz}, \mathrm{CDCl}_{3}\right)$

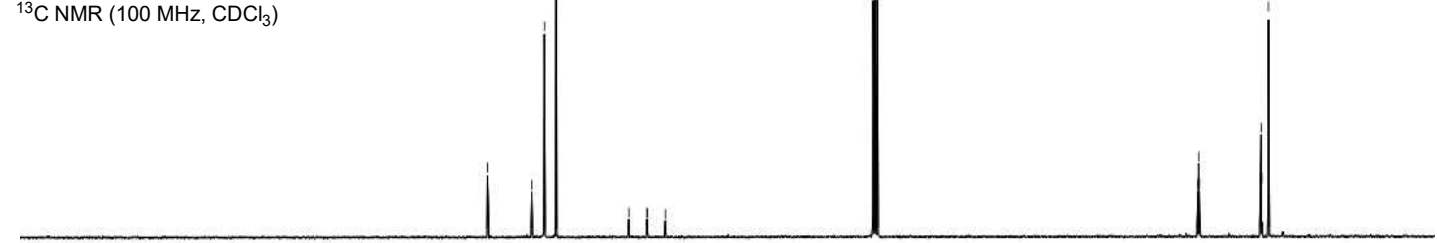

\begin{tabular}{llllllllllllllllllllllllllllllllllll}
\hline & 200 & 190 & 180 & 170 & 160 & 150 & 140 & 130 & 120 & 110 & 100 & 90 & 80 & 70 & 60 & 50 & 40 & 30 & 20 & 10 & 0 & -1
\end{tabular} 

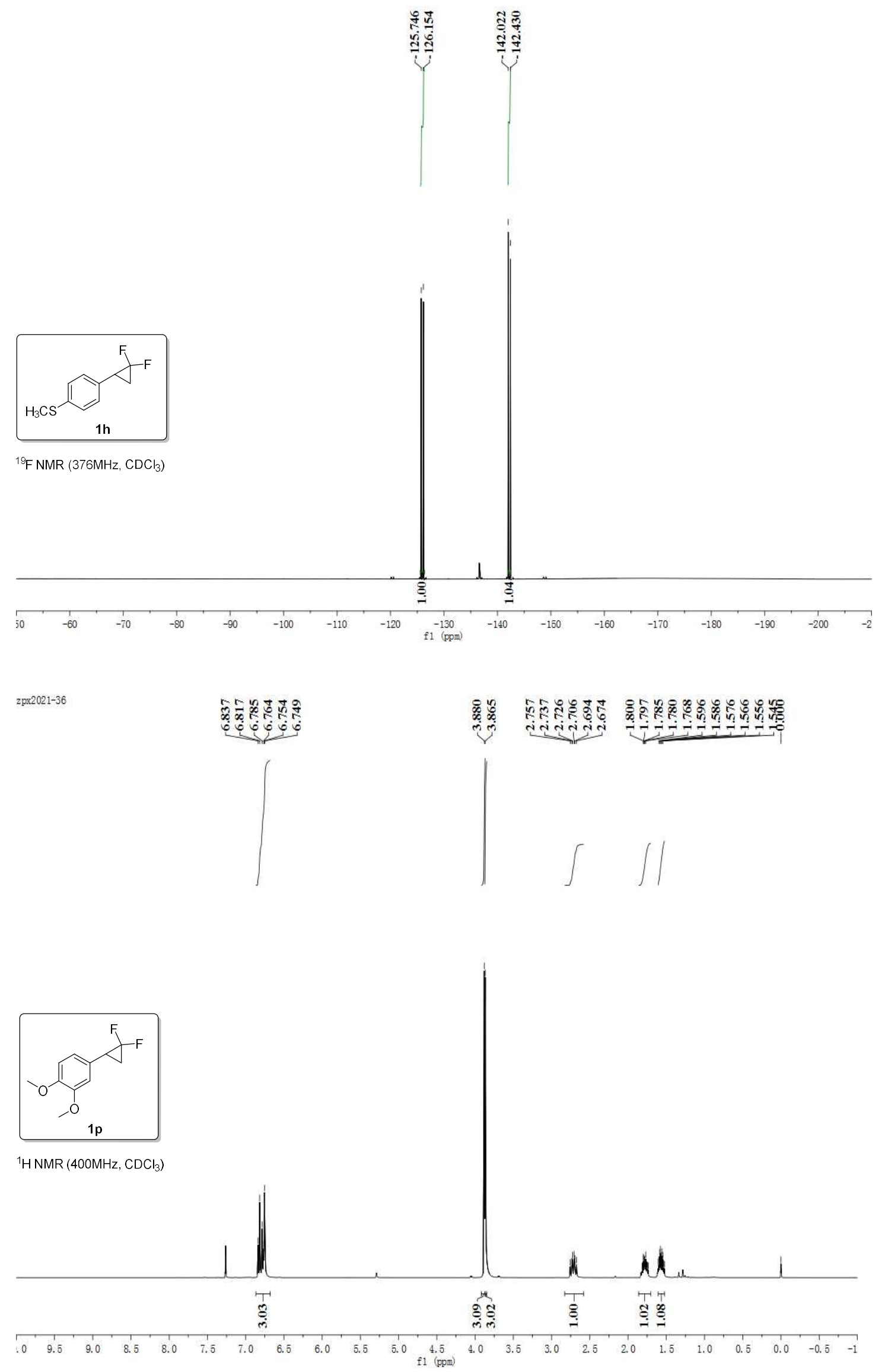

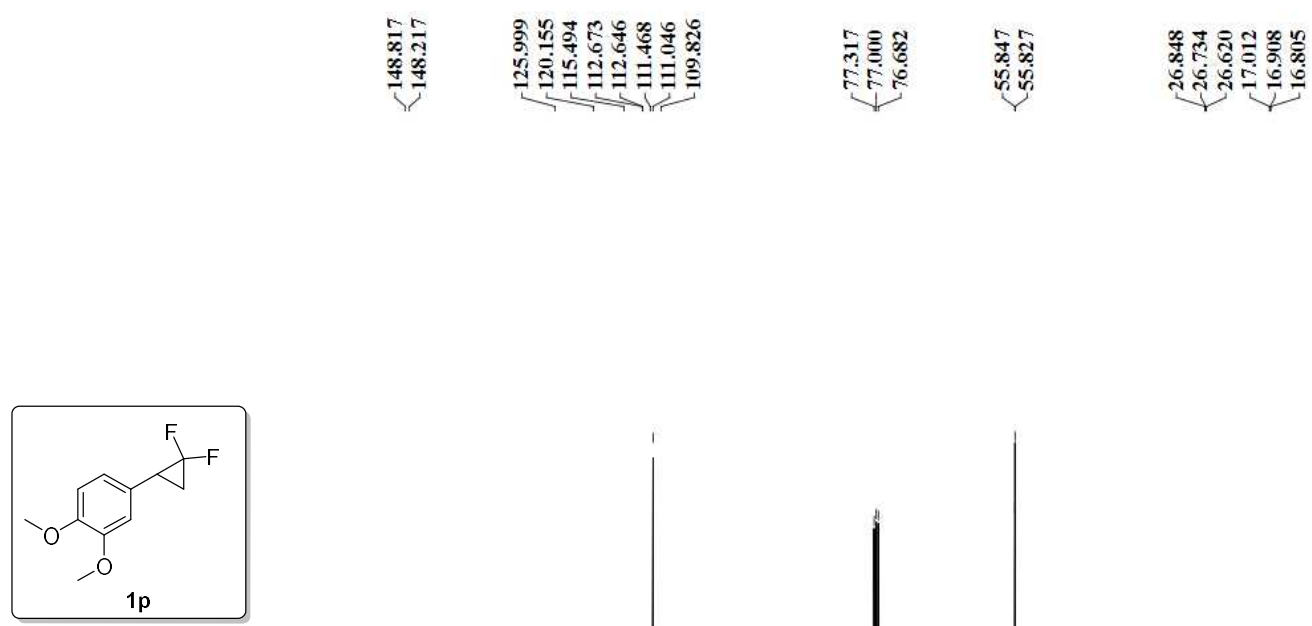

${ }^{13} \mathrm{C} \mathrm{NMR}\left(100 \mathrm{MHz}, \mathrm{CDCl}_{3}\right)$
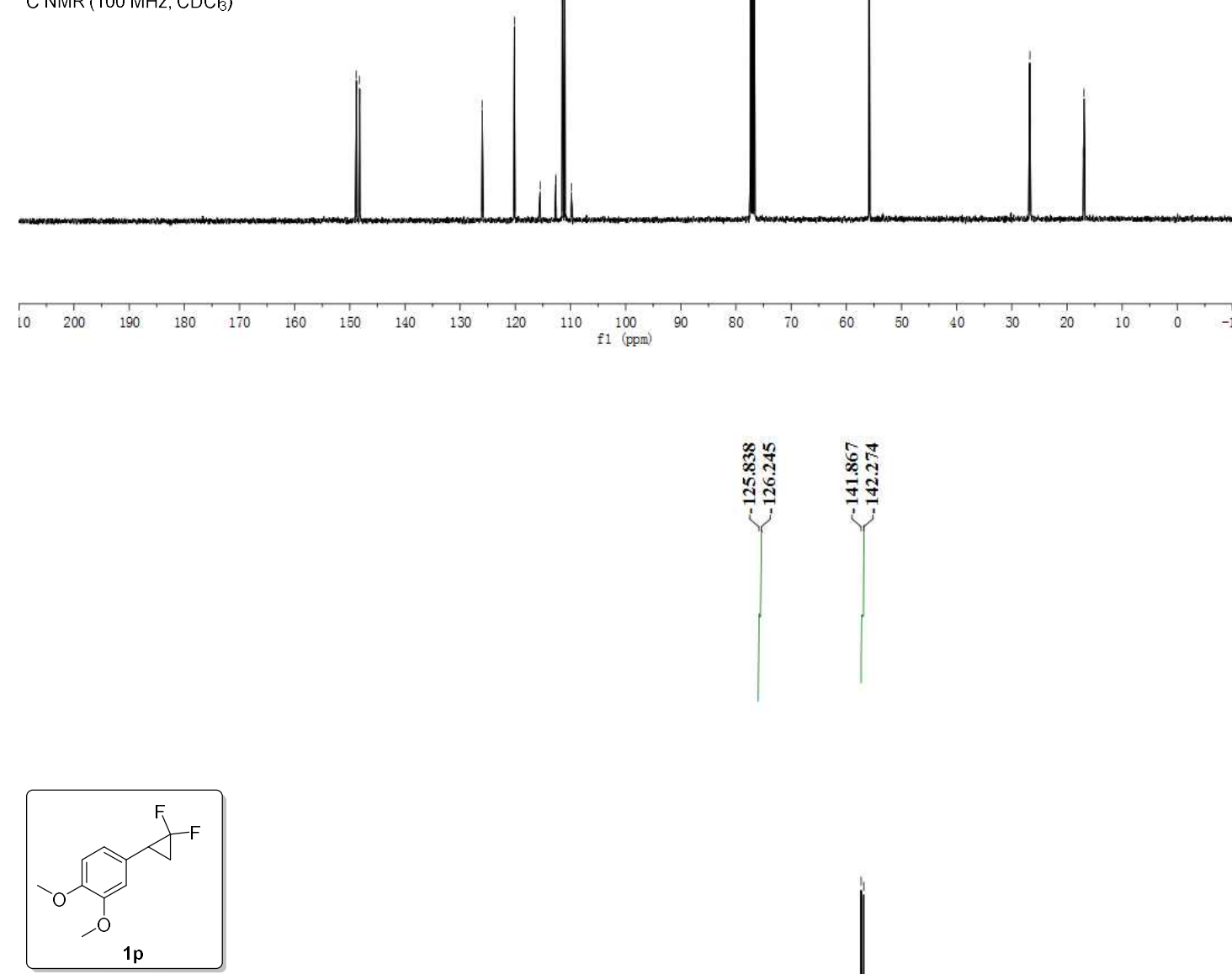

${ }^{1 \mathrm{C}} \mathrm{F} \mathrm{NMR}\left(376 \mathrm{MHz}, \mathrm{CDCl}_{3}\right)$

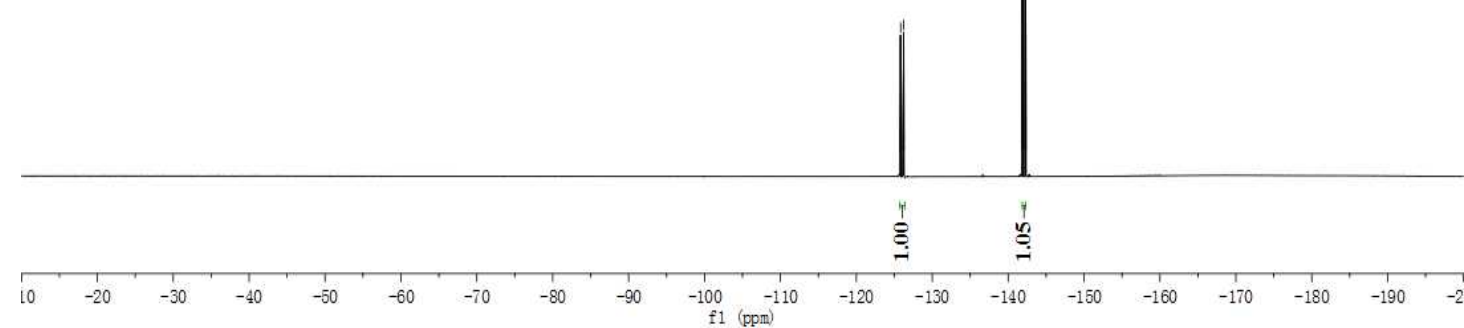




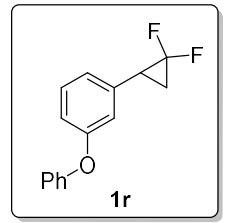

${ }^{1} \mathrm{H} \mathrm{NMR}\left(400 \mathrm{MHz}, \mathrm{CDCl}_{3}\right)$
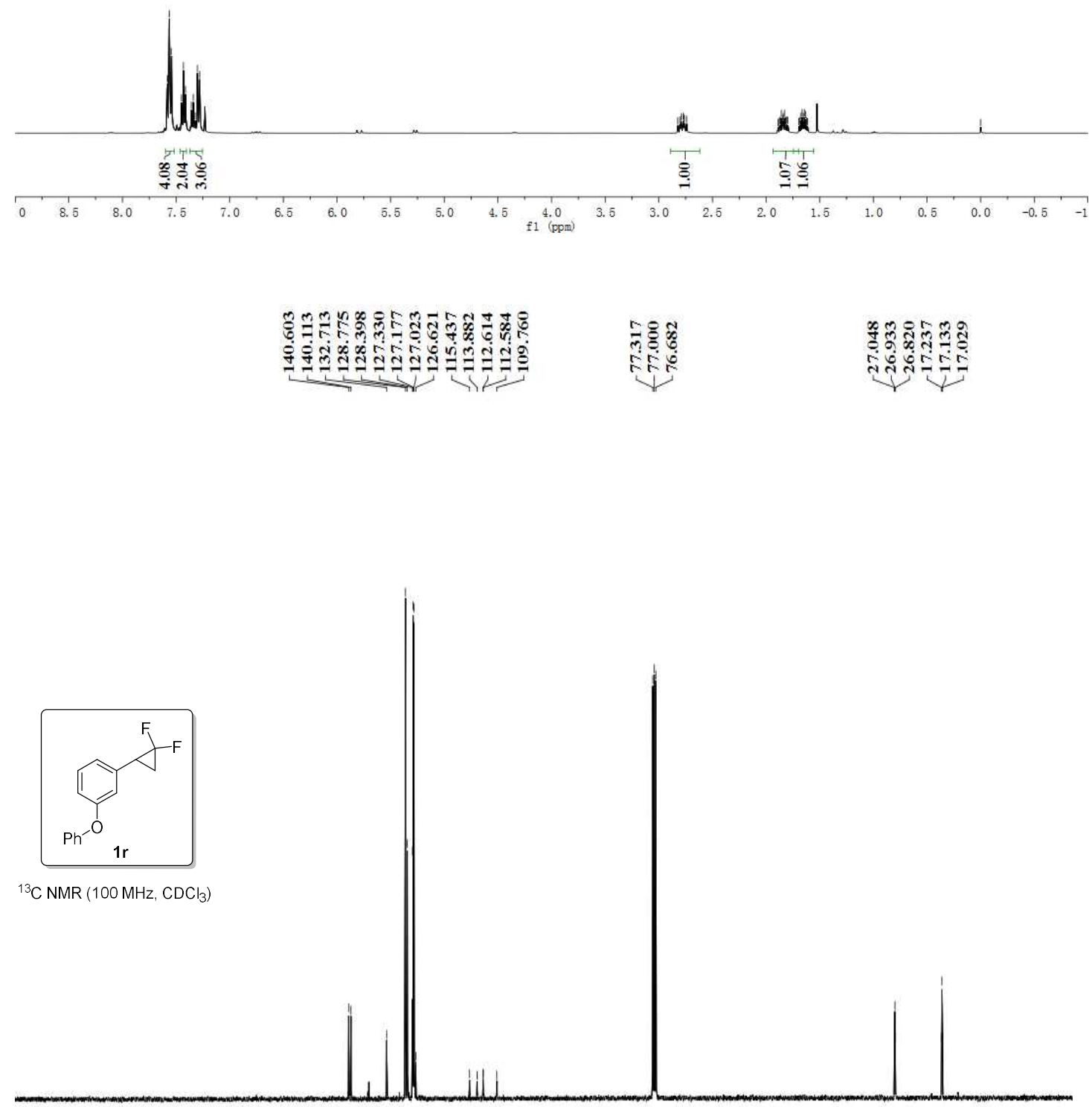

$\begin{array}{llllllllllll}1 . & 200 & 190 & 180 & 170 & 160 & 150 & 140 & 130 & 120 & 110 & 100\end{array}$ 

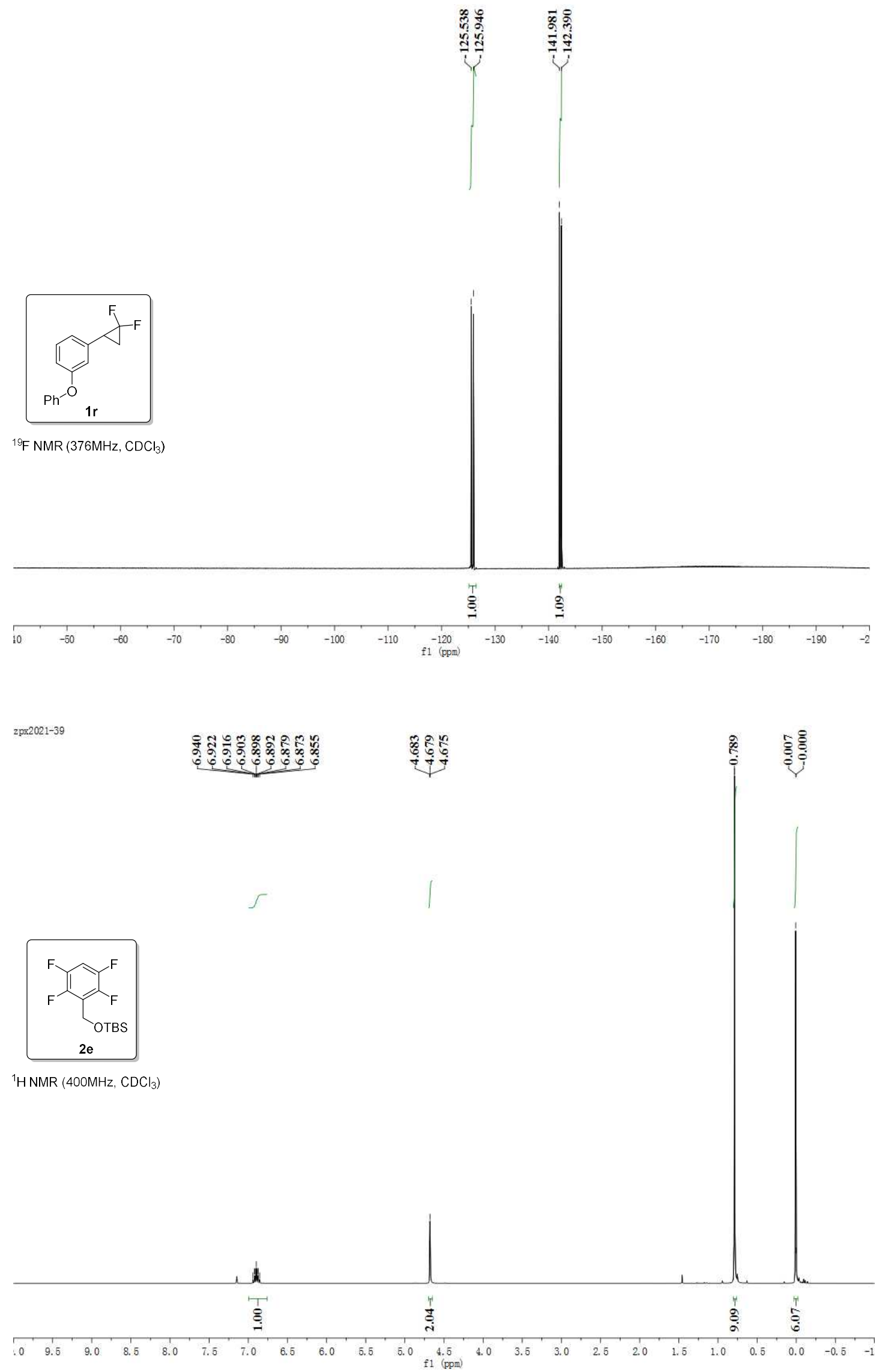


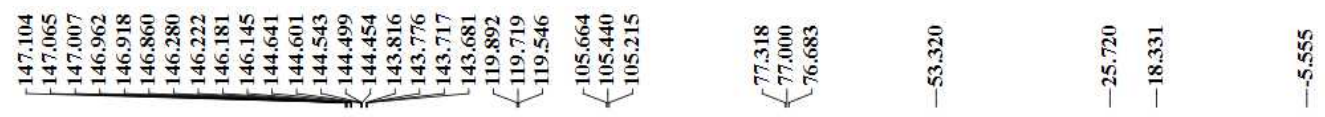

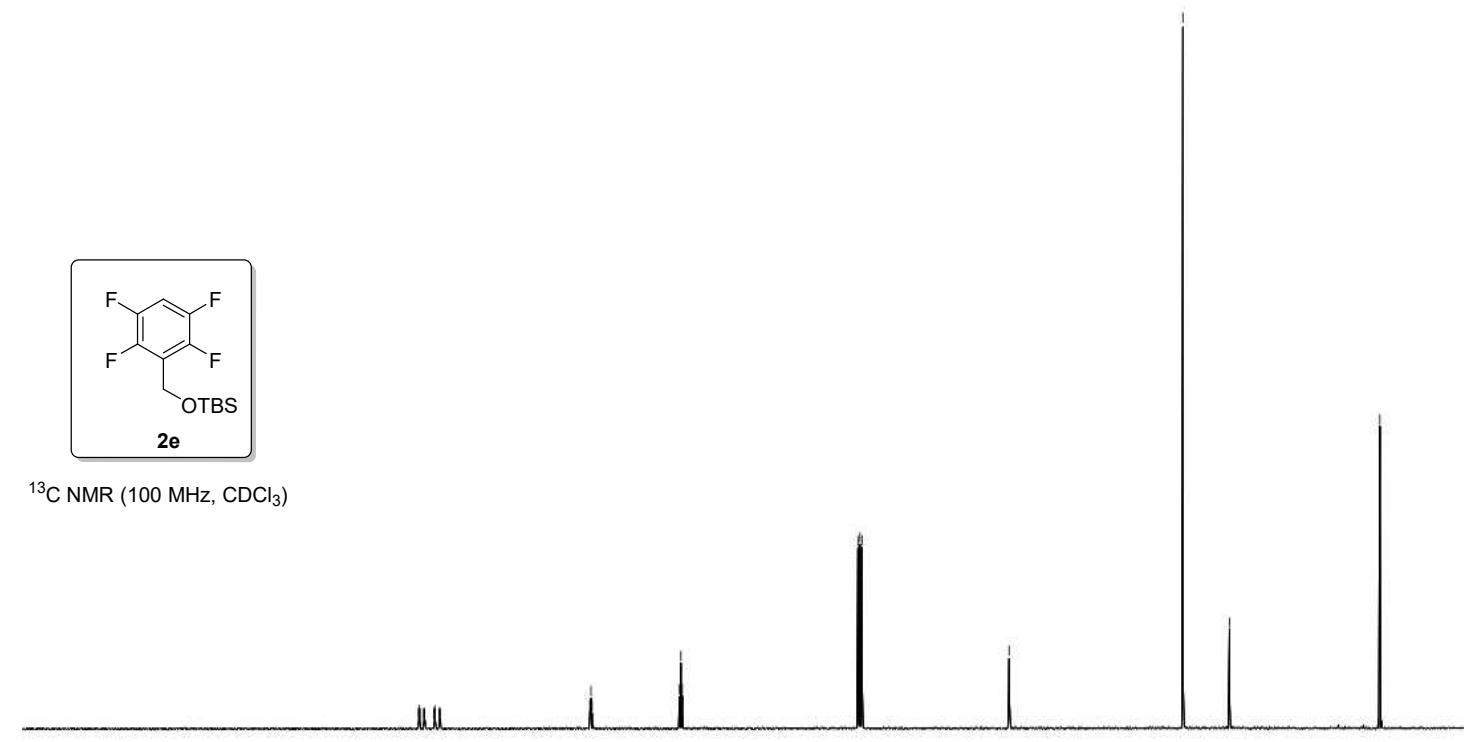

\begin{tabular}{lllllllllllllllllllllllllllll}
\hline 0 & 200 & 190 & 180 & 170 & 160 & 150 & 140 & 130 & 120 & 110 & 100 & 90 & 80 & 70 & 60 & 50 & 40 & 30 & 20 & 10 & 0 & -10
\end{tabular}

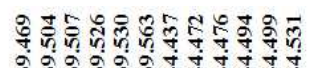

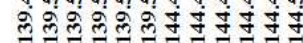

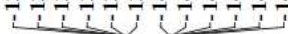
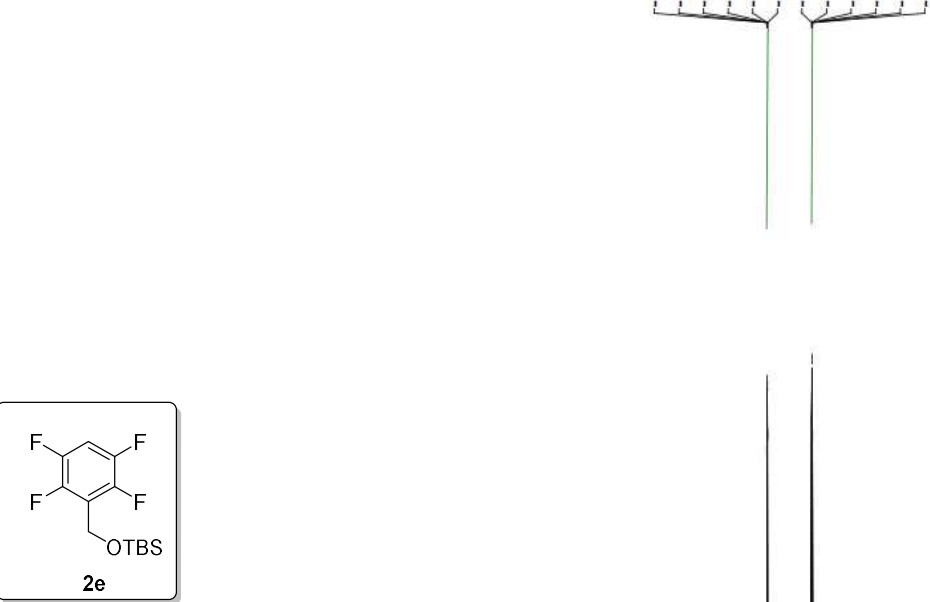

${ }^{19} \mathrm{~F} \mathrm{NMR}\left(376 \mathrm{MHz}, \mathrm{CDCl}_{3}\right)$

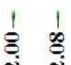

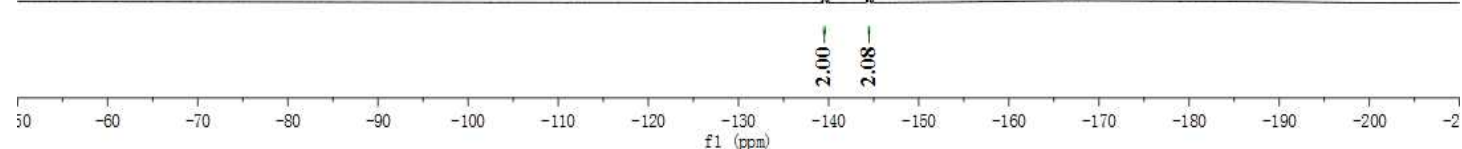




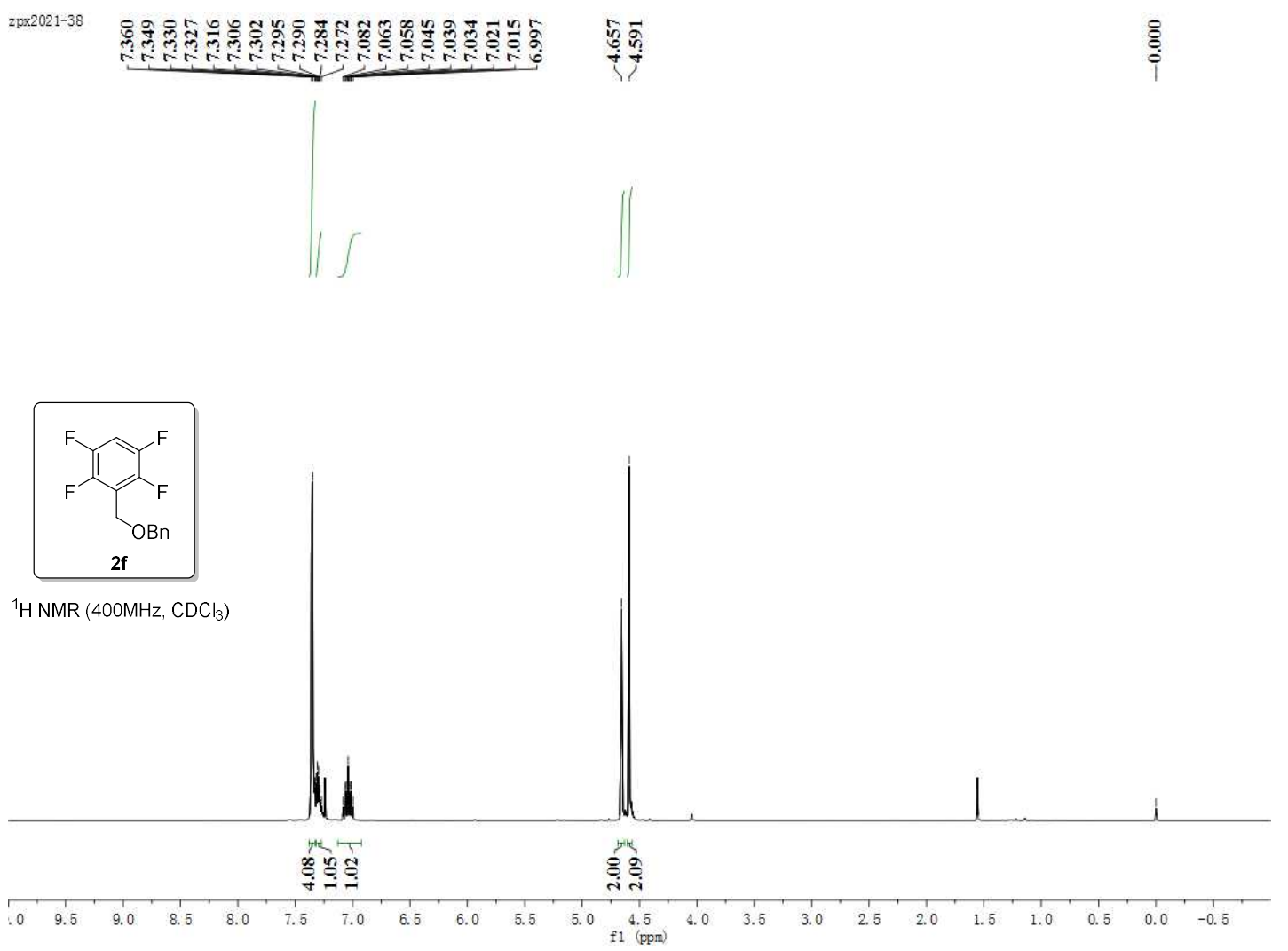

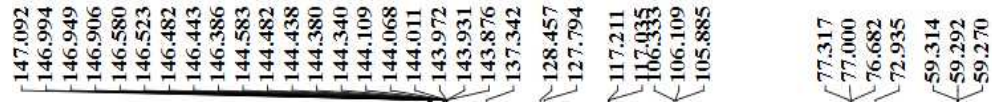

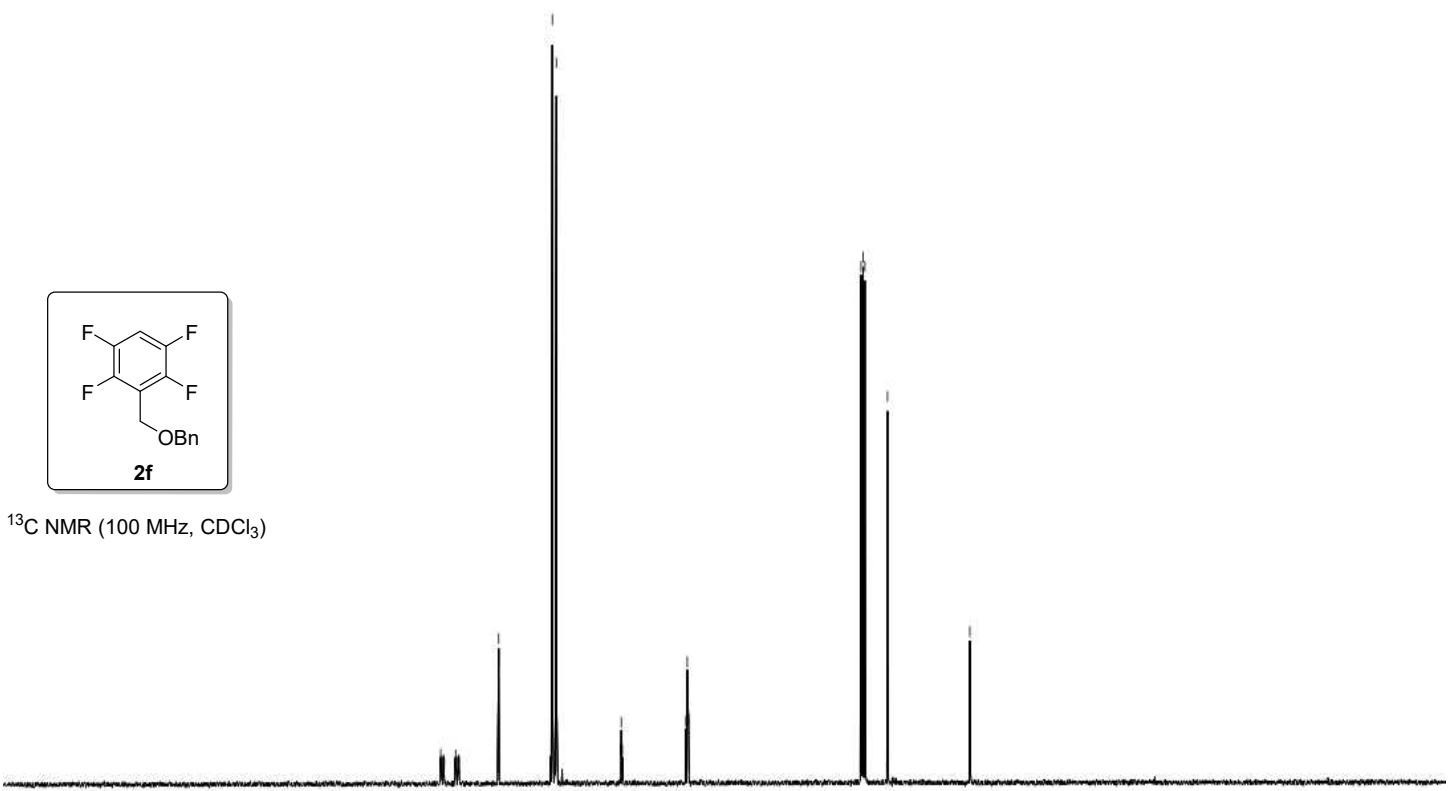

$\begin{array}{lllllllllllllllllllllllll}210 & 200 & 190 & 180 & 170 & 160 & 150 & 140 & 130 & 120 & 110 & 100 & 90 & 80 & 70 & 60 & 50 & 40 & 30 & 20 & 10 & 0 & -10\end{array}$ 

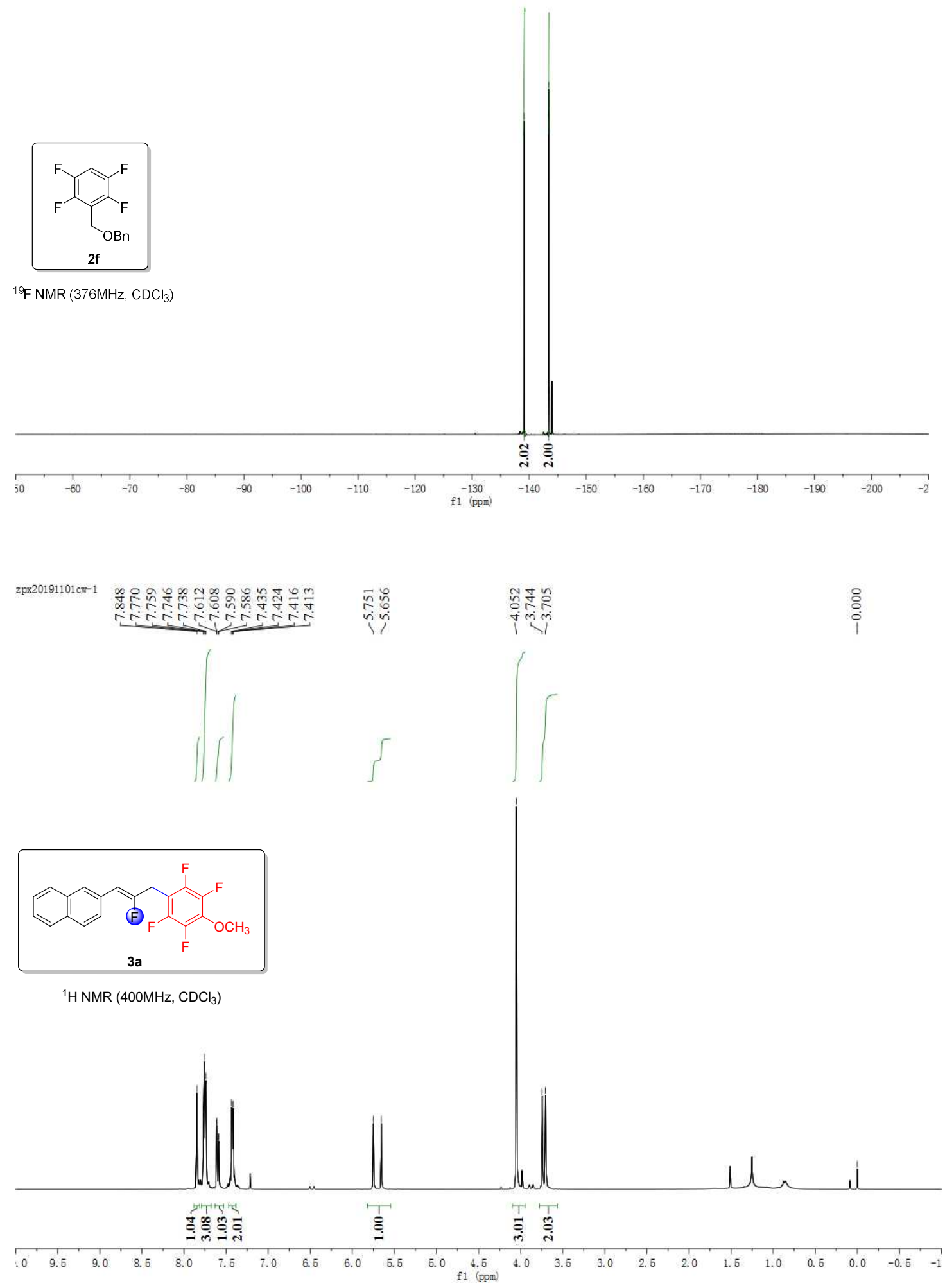


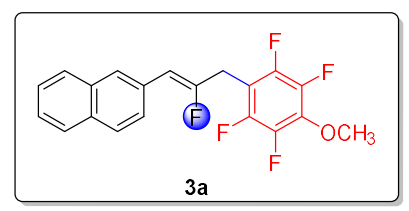

$\left.{ }^{13} \mathrm{C} \mathrm{NMR} \mathrm{(100} \mathrm{MHz,} \mathrm{CDCl}_{3}\right)$
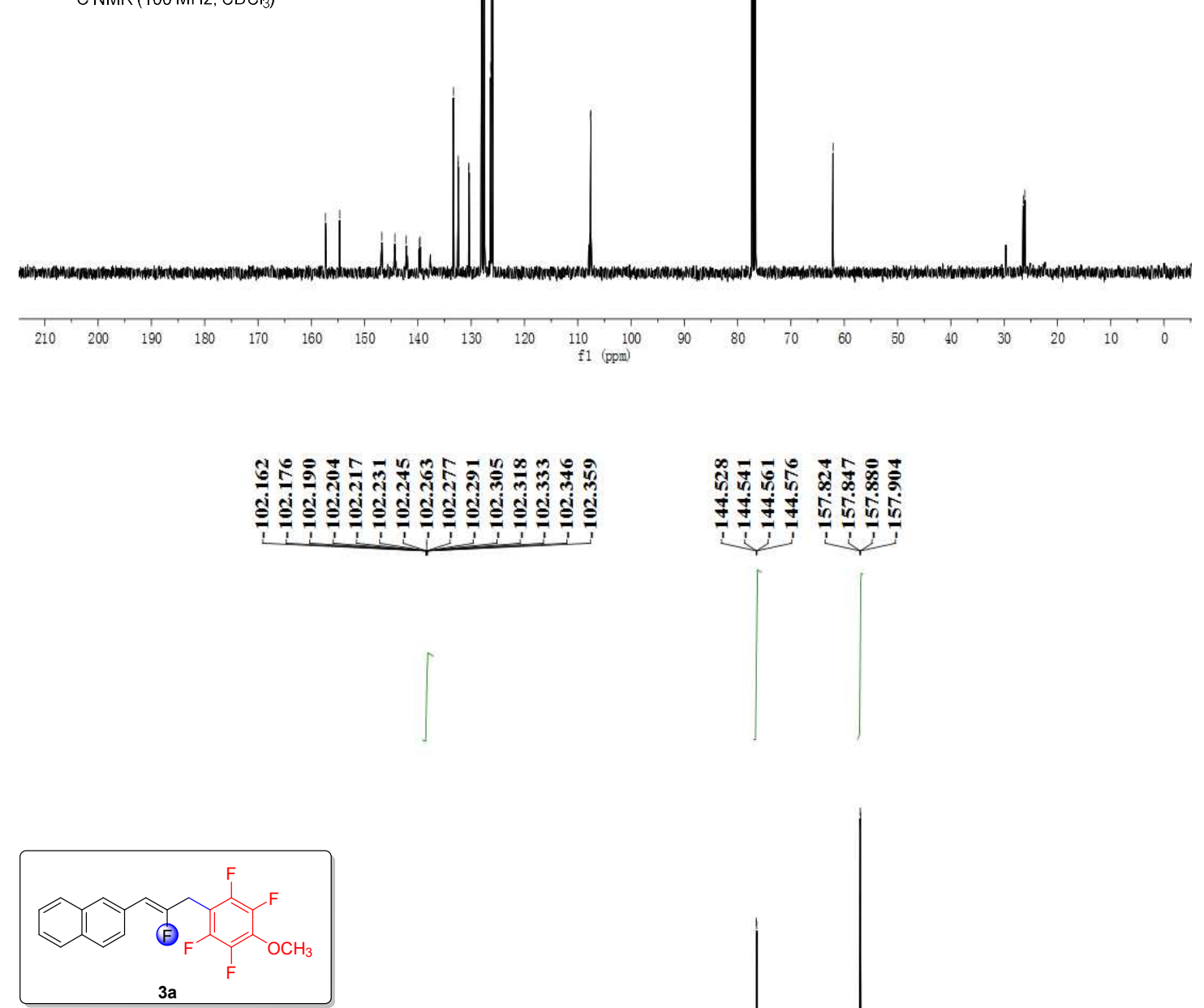

${ }^{19} \mathrm{~F} \mathrm{NMR}\left(376 \mathrm{MHz}, \mathrm{CDCl}_{3}\right)$

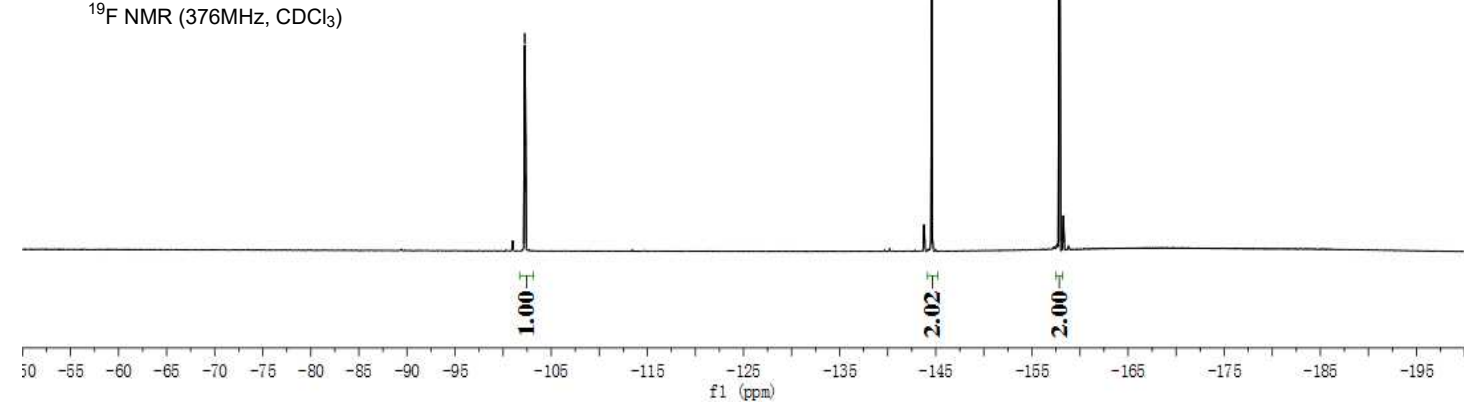



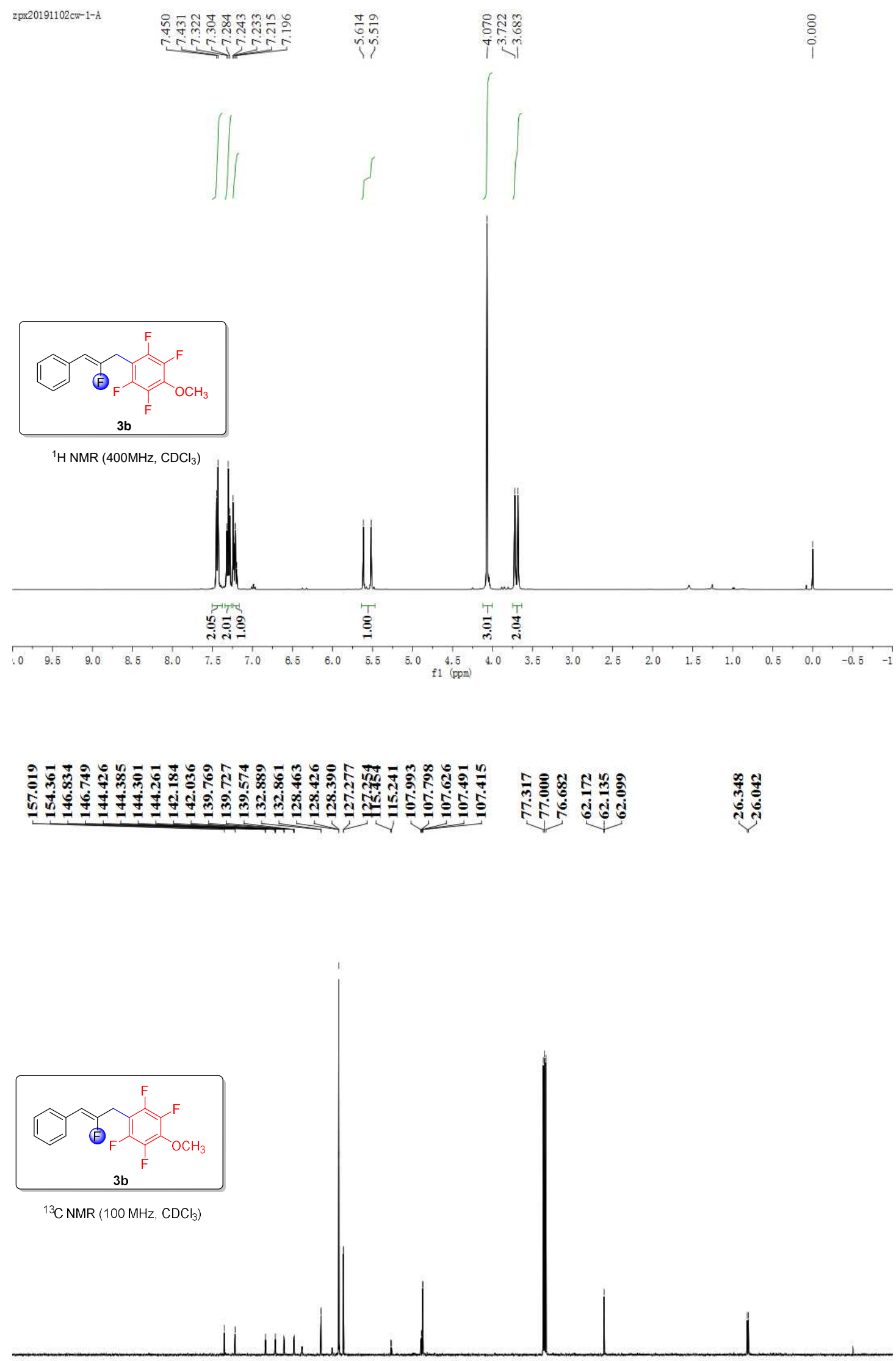

\begin{tabular}{lllllllllllllllllllllllll}
\hline 0 & 200 & 190 & 180 & 170 & 160 & 150 & 140 & 130 & 120 & 110 & 100 & 90 & 80 & 70 & 60 & 50 & 40 & 30 & 20 & 10 & 0 & -1
\end{tabular} 

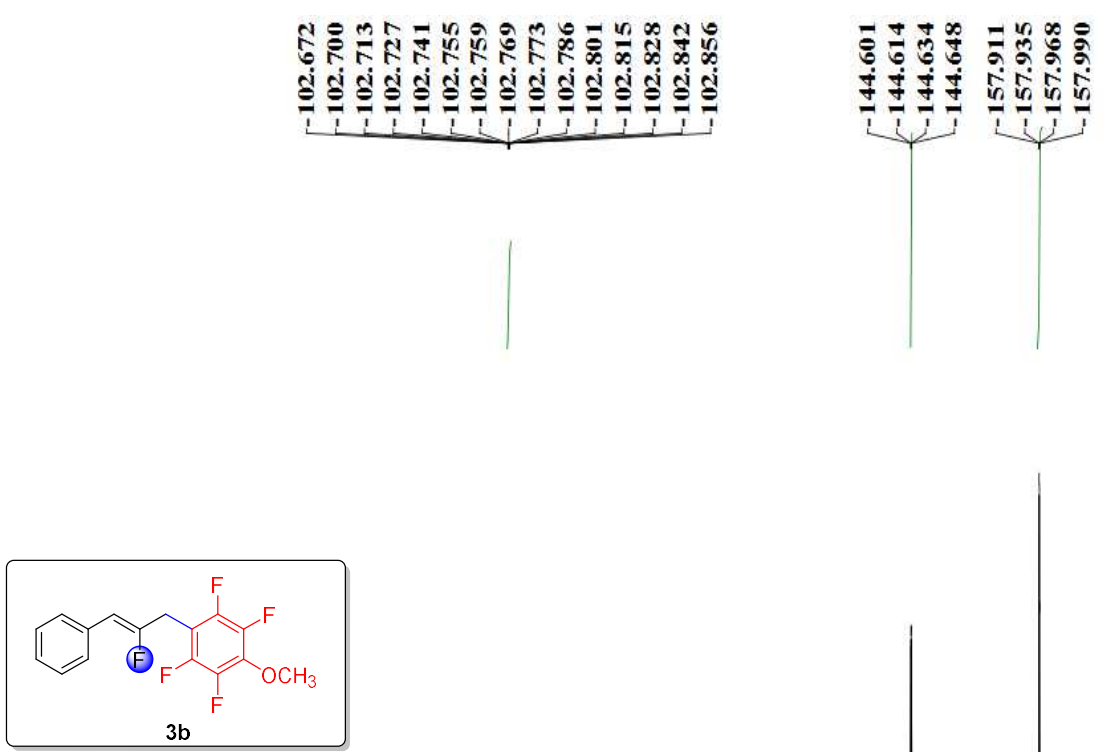

${ }^{19} \mathrm{~F} \mathrm{NMR}\left(376 \mathrm{MHz}, \mathrm{CDCl}_{3}\right)$
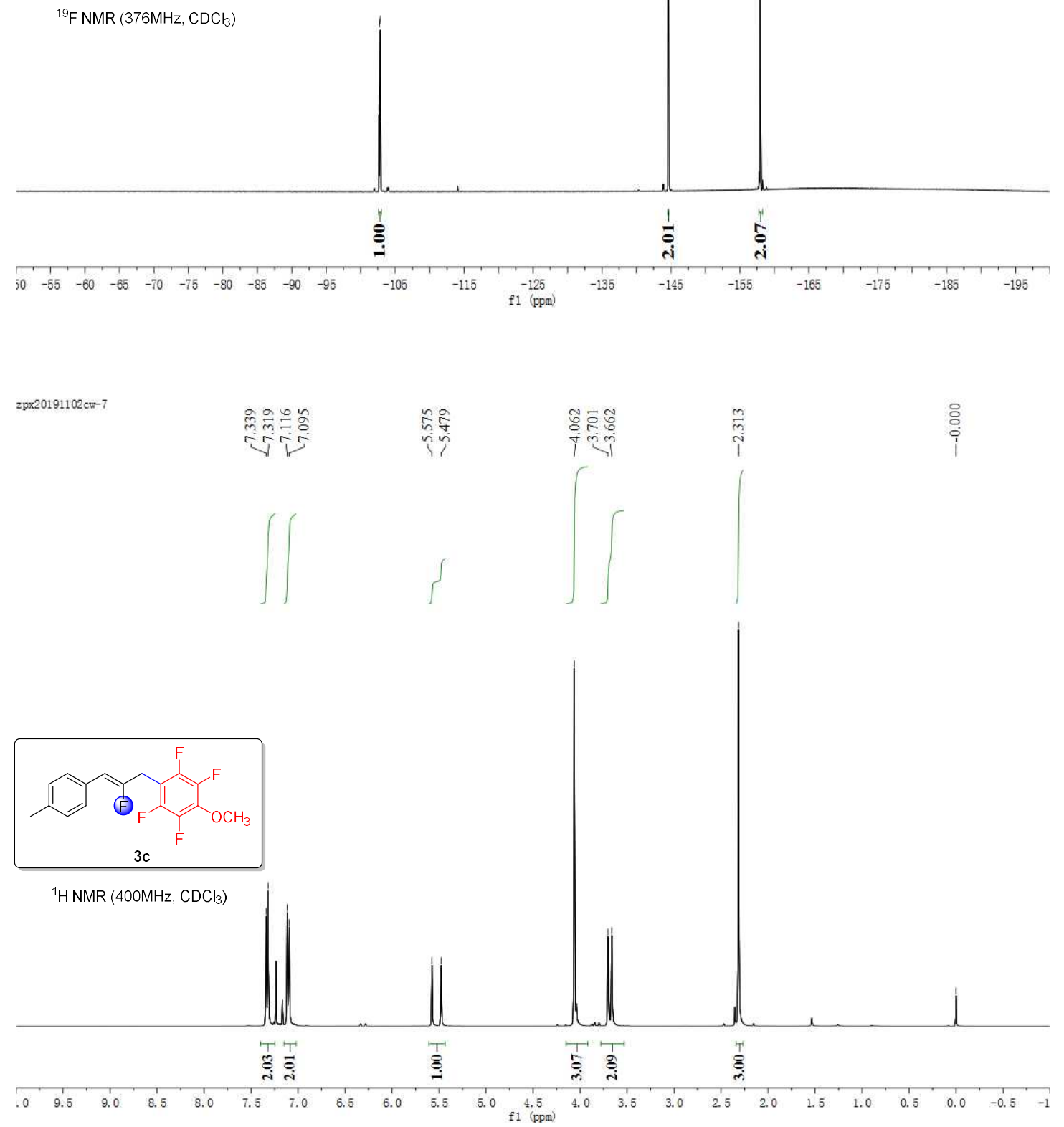


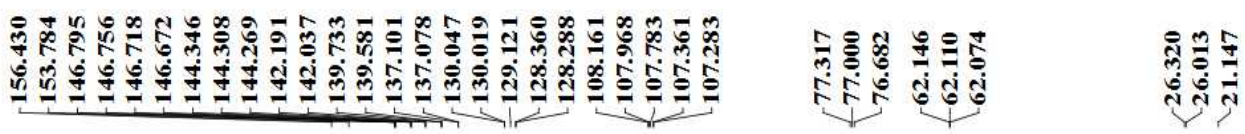

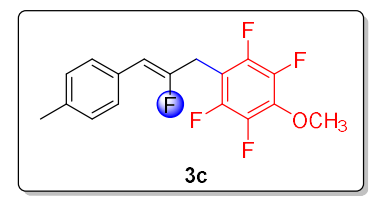

$\left.{ }^{13} \mathrm{C} \mathrm{NMR} \mathrm{(100} \mathrm{MHz} \mathrm{CDCl}_{3}\right)$

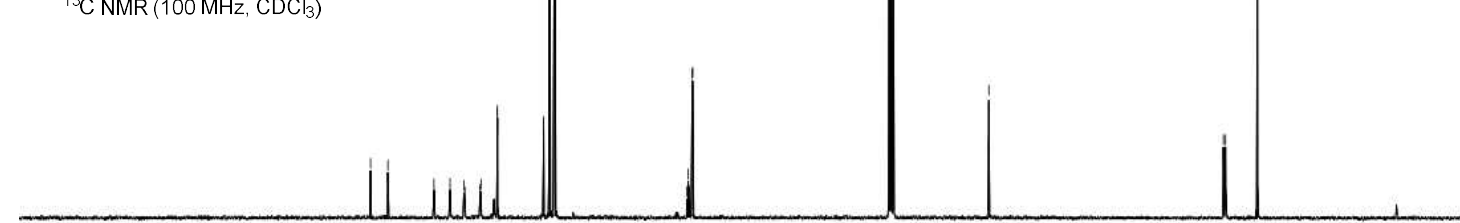

\begin{tabular}{llllllllllllllllllllllll}
\hline 0 & 200 & 190 & 180 & 170 & 160 & 150 & 140 & 130 & 120 & 110 & 100 & 90 & 80 & 70 & 60 & 50 & 40 & 30 & 20 & 10 & 0 & -1
\end{tabular}

zpr $20191102 \mathrm{cw}-7$
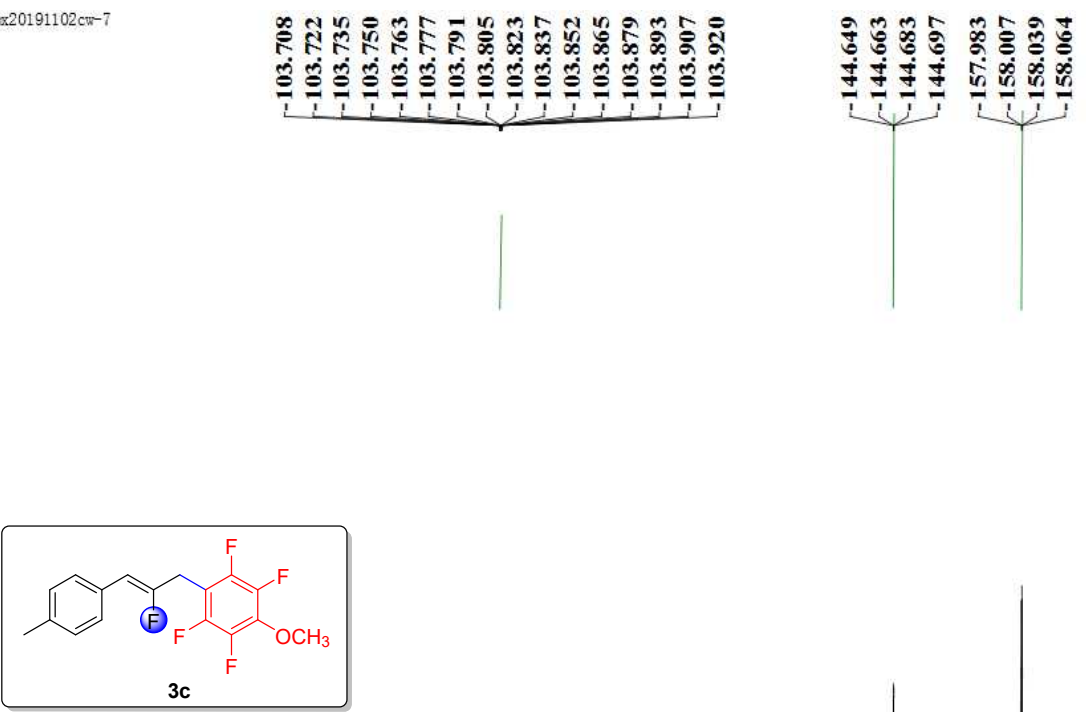

${ }^{19} \mathrm{~F} \mathrm{NMR}\left(376 \mathrm{MHz}, \mathrm{CDCl}_{3}\right)$

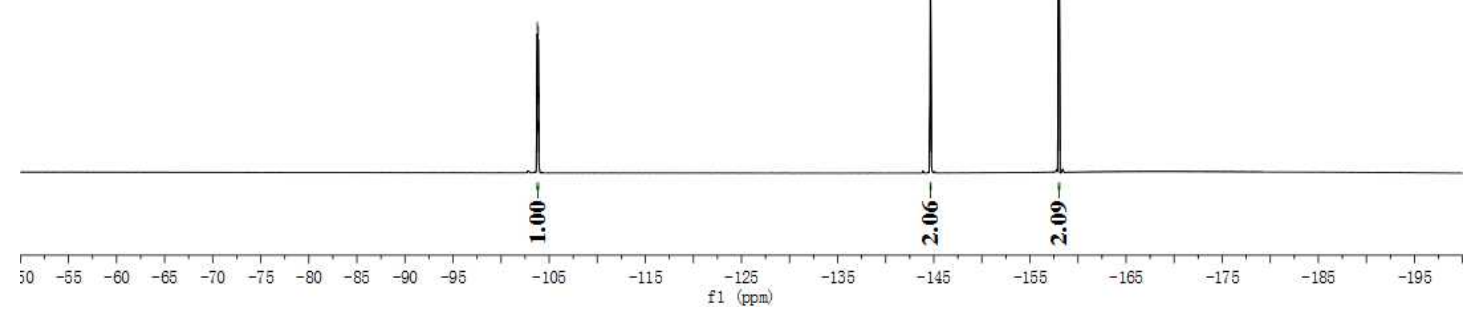



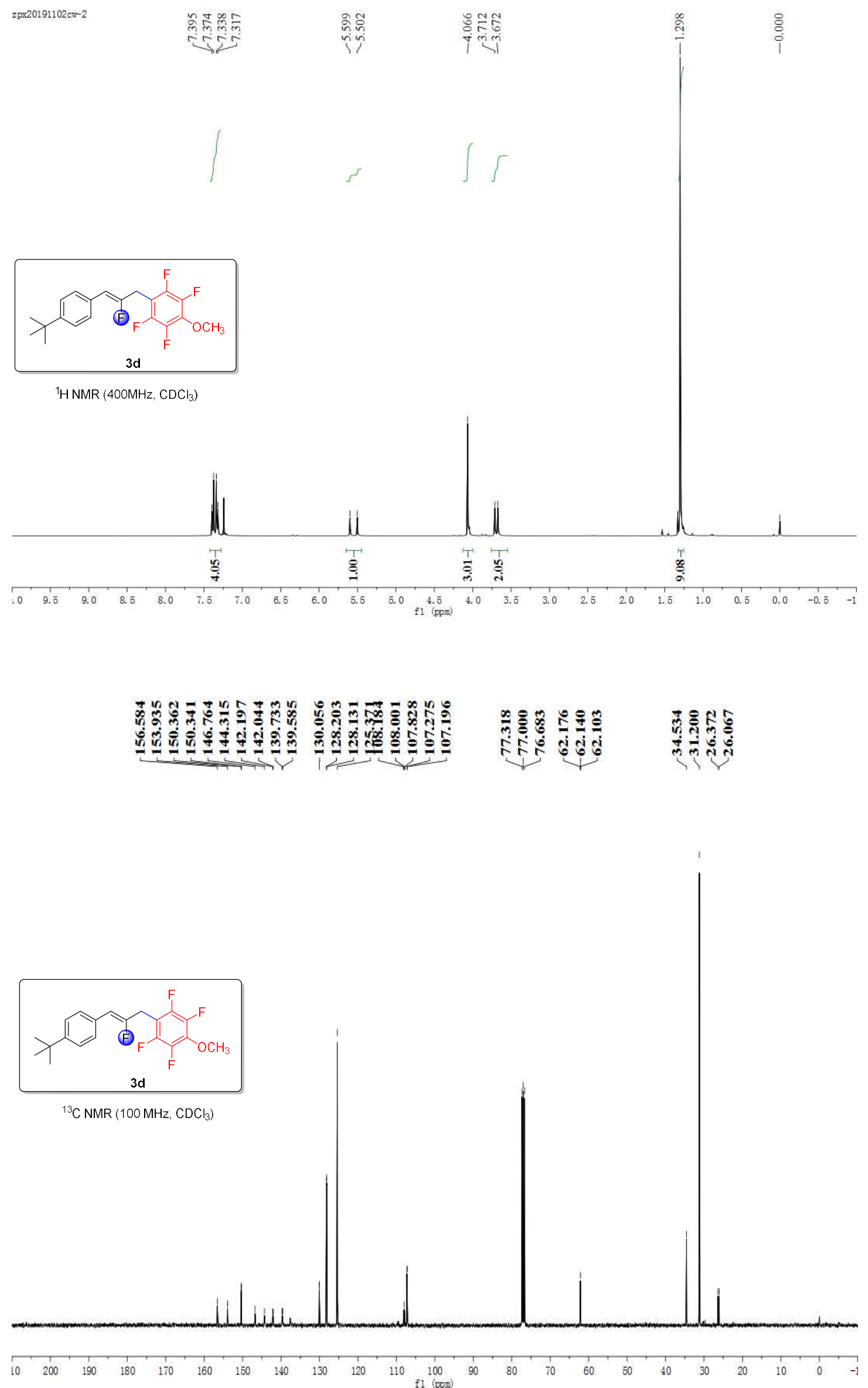
zpx20191102 cw-2
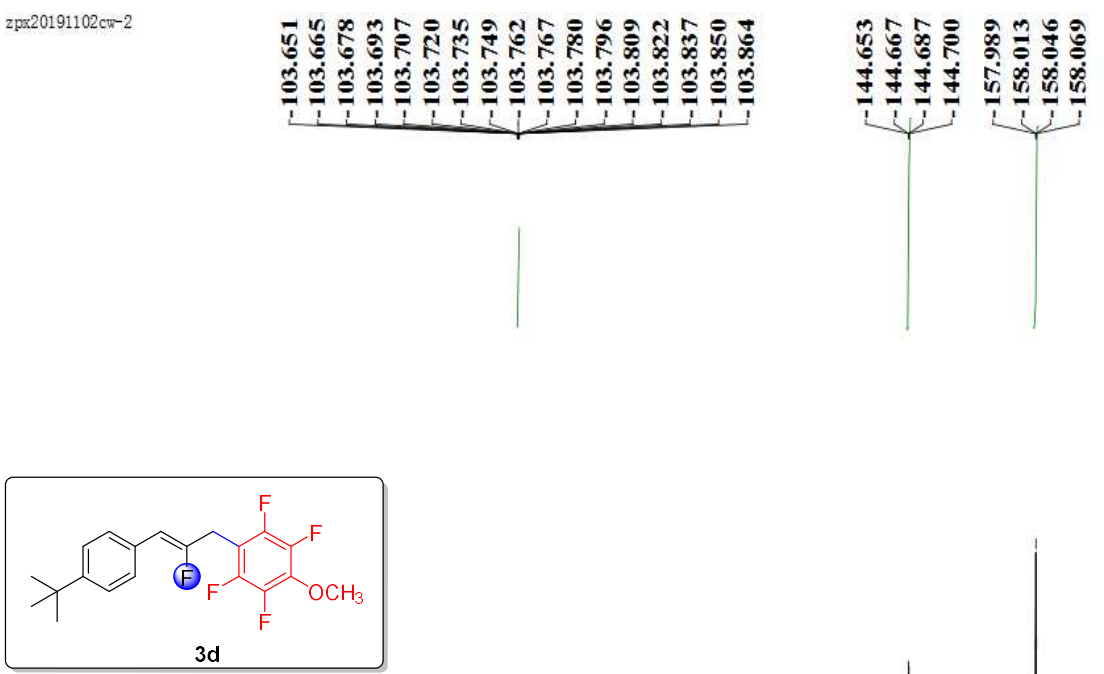

${ }^{19} \mathrm{~F} \mathrm{NMR}\left(376 \mathrm{MHz}, \mathrm{CDCl}_{3}\right)$
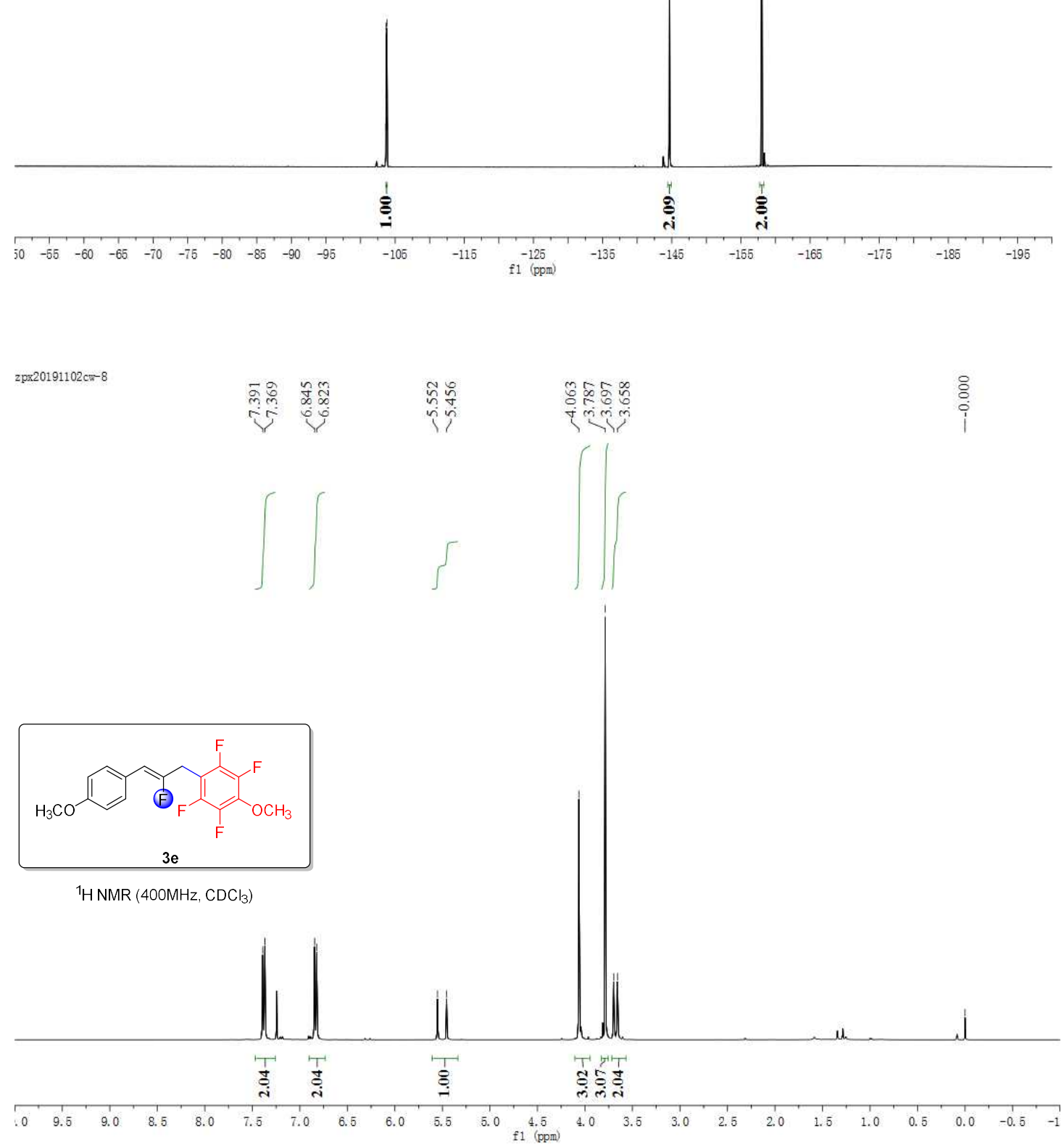


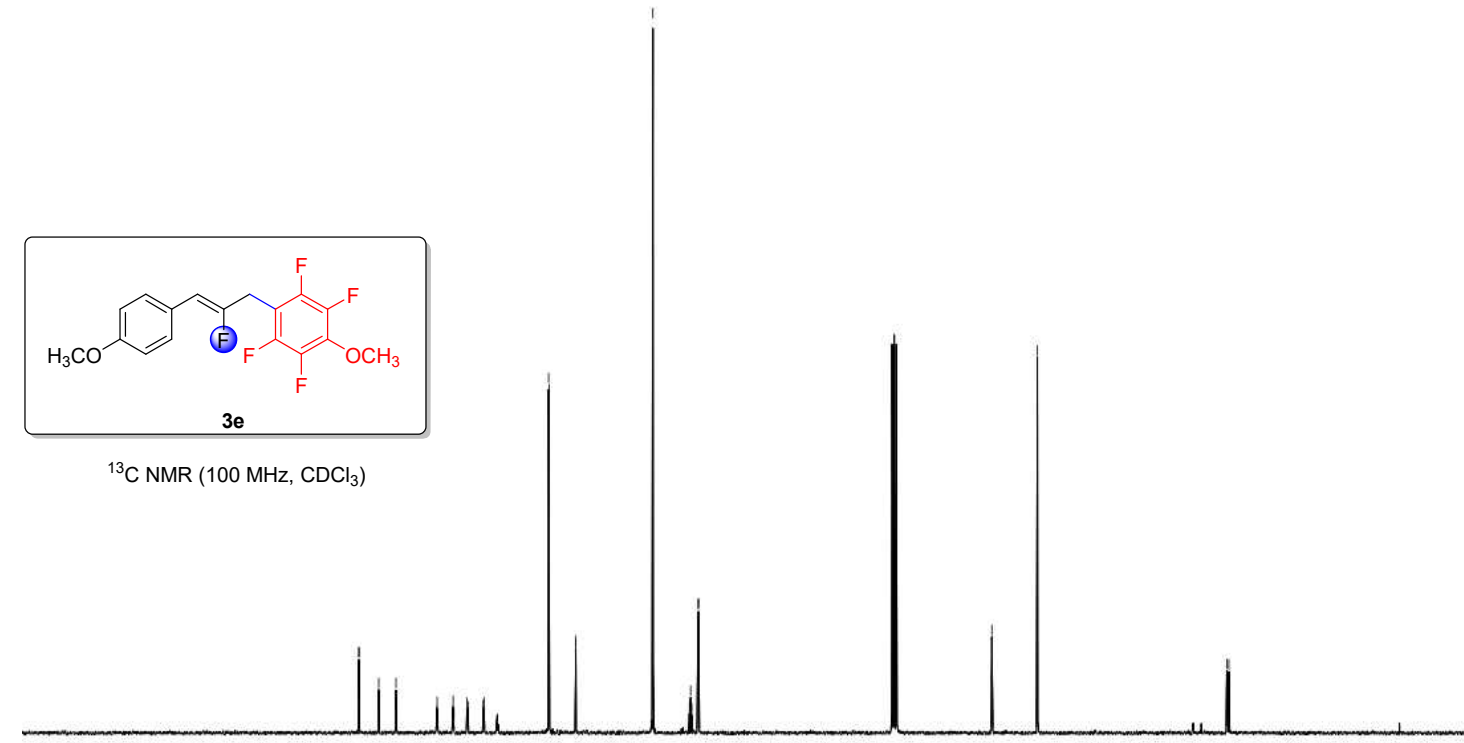

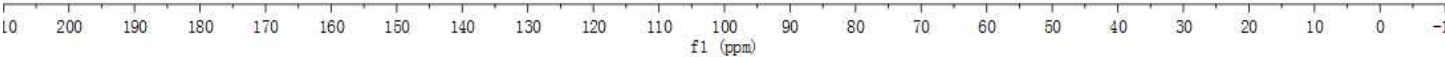

zpx20191102cw-8
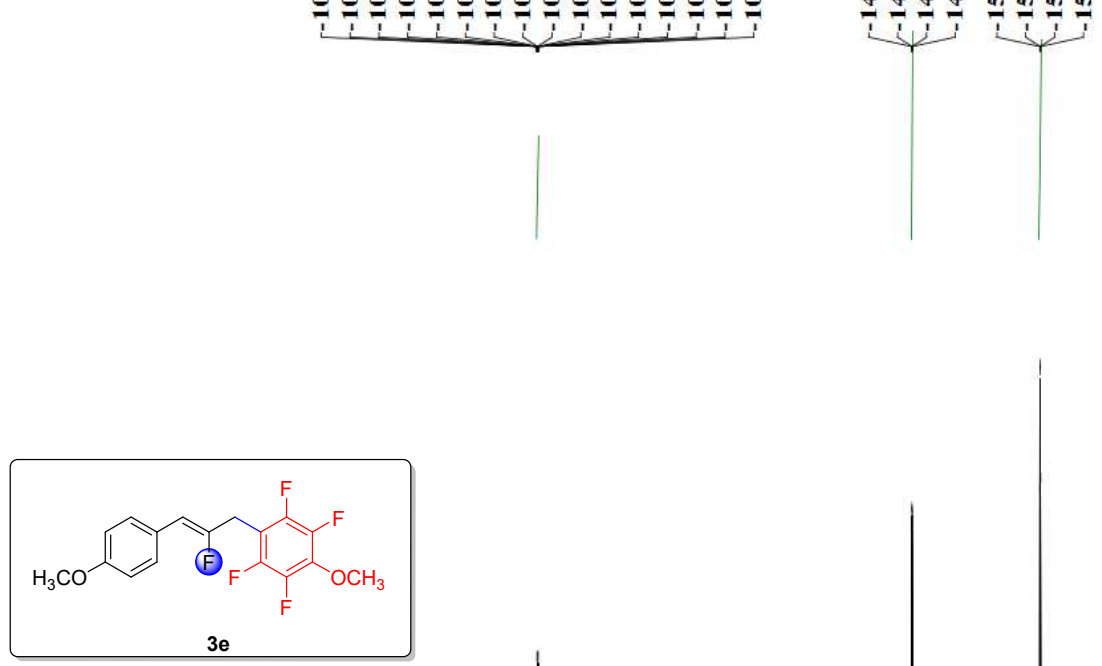

${ }^{19} \mathrm{~F} \mathrm{NMR}\left(376 \mathrm{MHz}, \mathrm{CDCl}_{3}\right)$

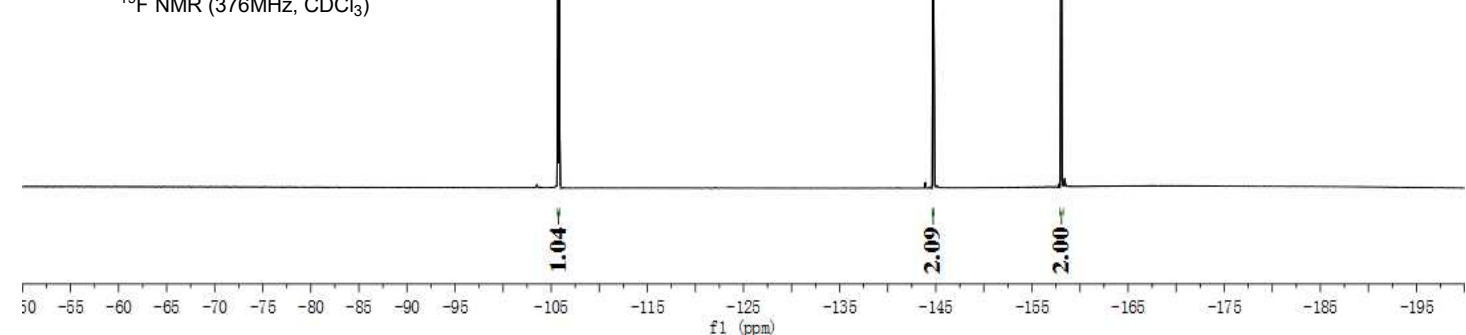



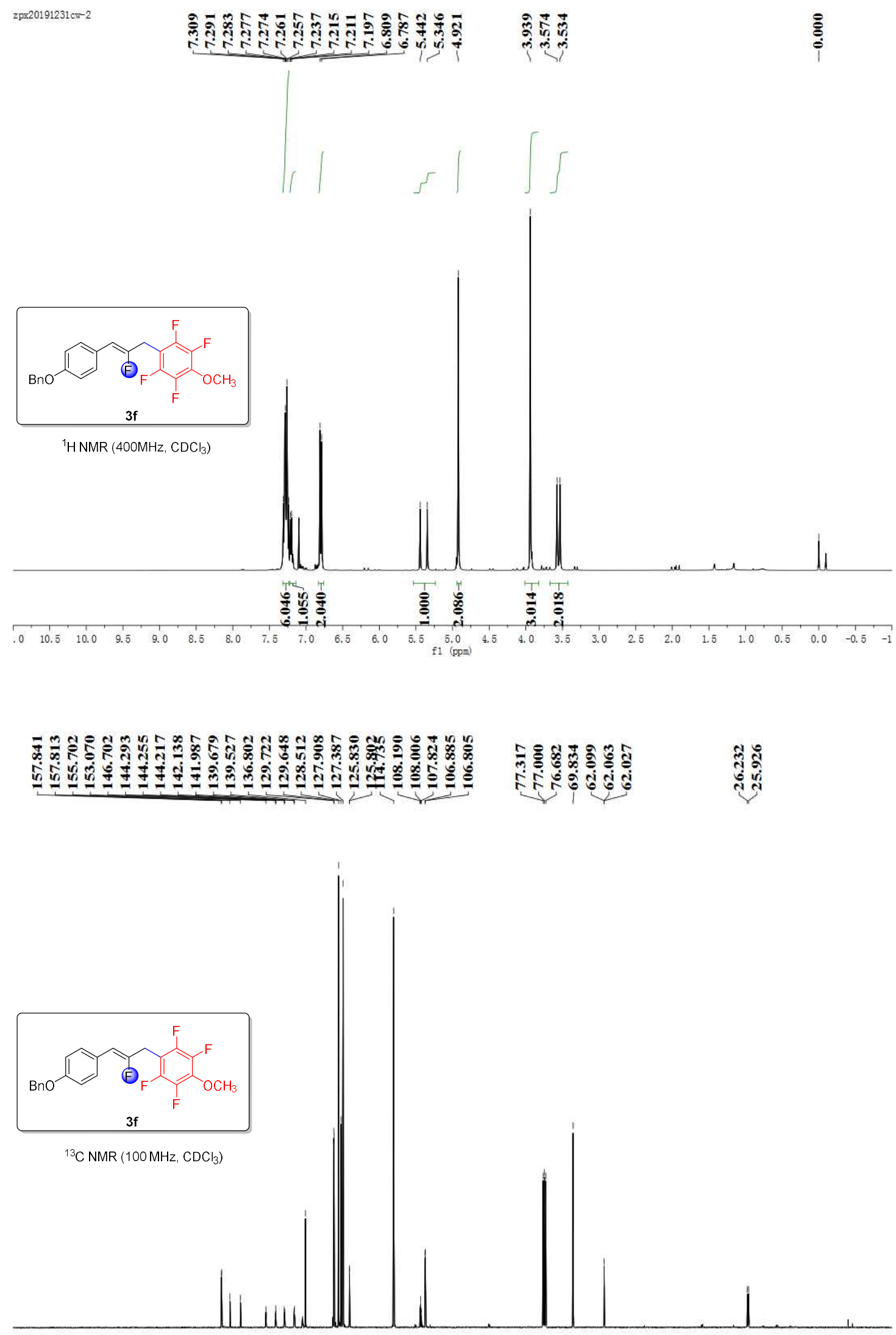

$\begin{array}{llllllllllllllllllllllllllllllllllll}10 & 200 & 190 & 180 & 170 & 160 & 150 & 140 & 130 & 120 & 110 & 100 & 90 & 80 & 70 & 60 & 50 & 40 & 30 & 20 & 10 & 0 & -1\end{array}$ 


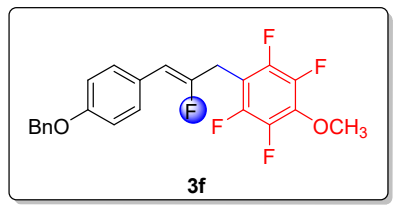

${ }^{19} \mathrm{~F} \mathrm{NMR}\left(376 \mathrm{MHz}, \mathrm{CDCl}_{3}\right)$
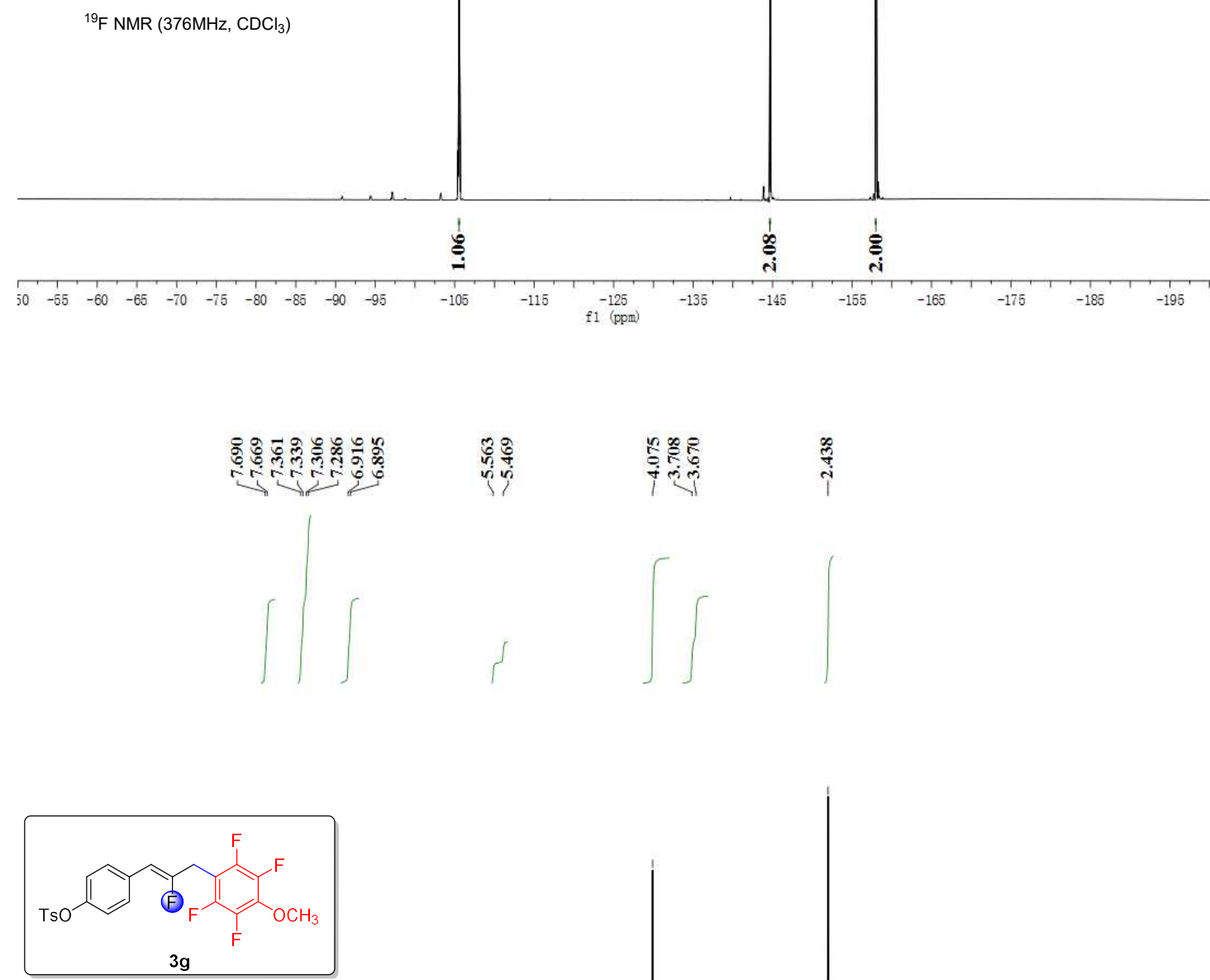

${ }^{1} \mathrm{H} \mathrm{NMR}\left(400 \mathrm{MHz}^{\left.-\mathrm{CDC}_{3}\right)}\right.$

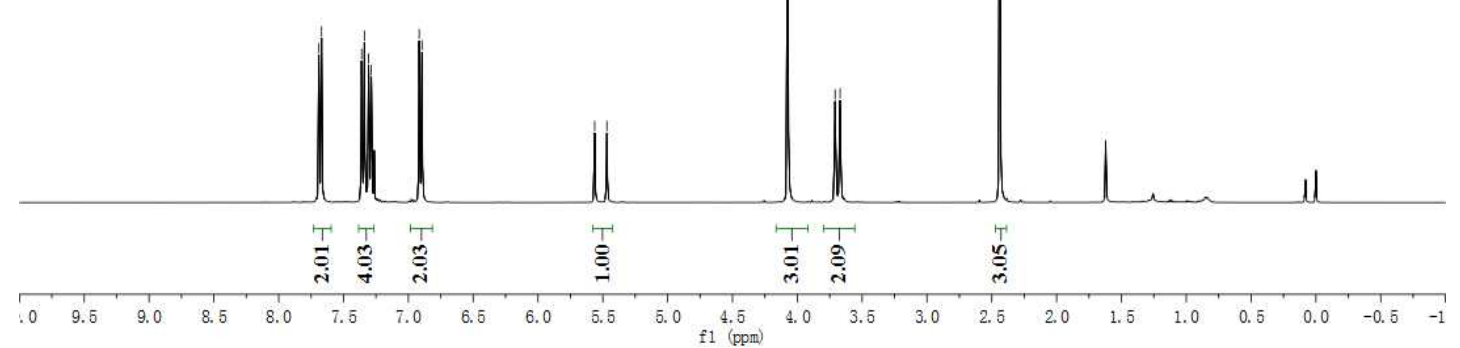




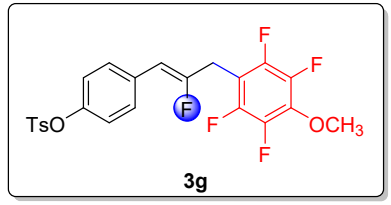

${ }^{13} \mathrm{C}$ NMR (100 MHz, $\mathrm{CDCl}_{3}$ )
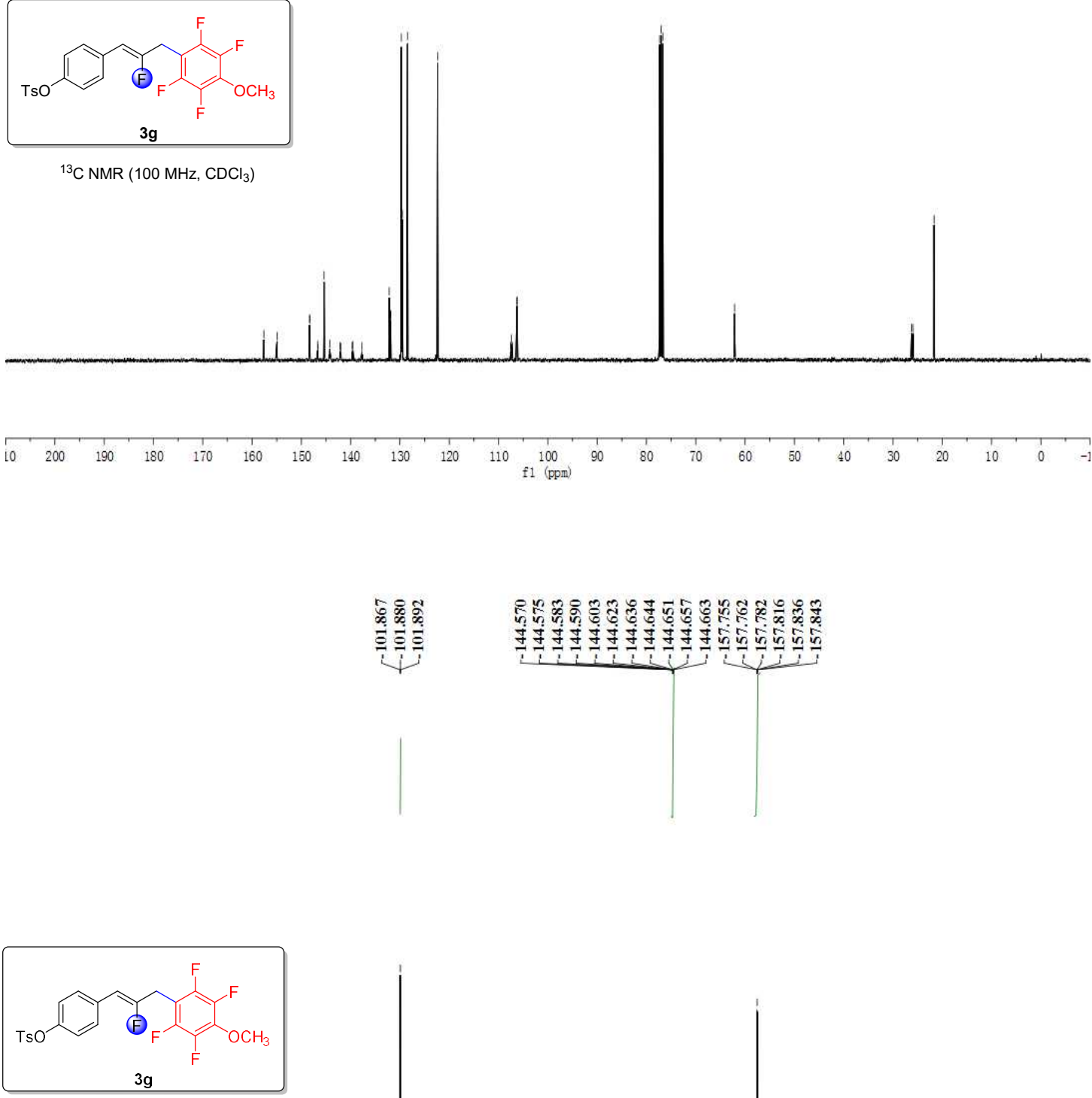

${ }^{19} \mathrm{~F} \mathrm{NMR}\left(376 \mathrm{MHz}, \mathrm{CDCl}_{3}\right)$

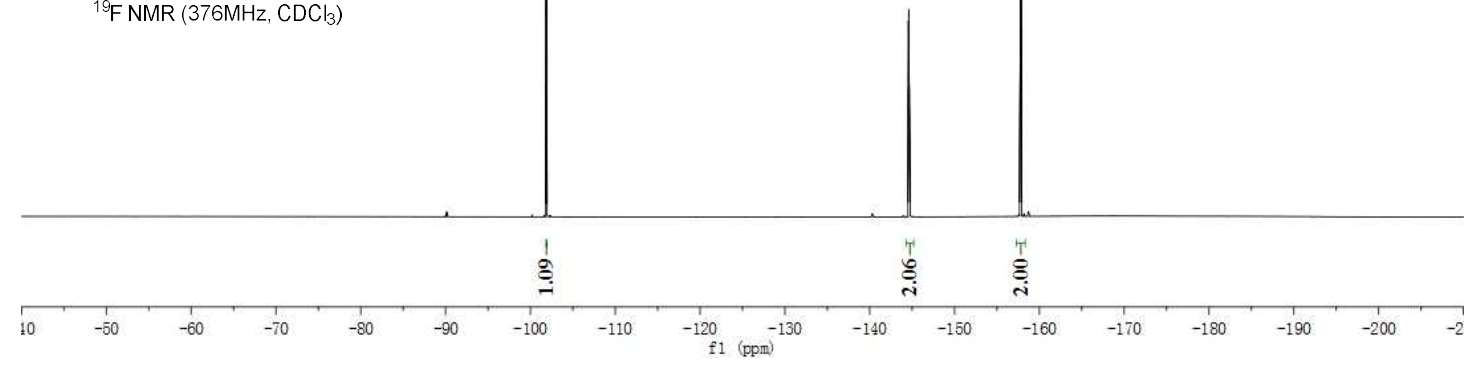




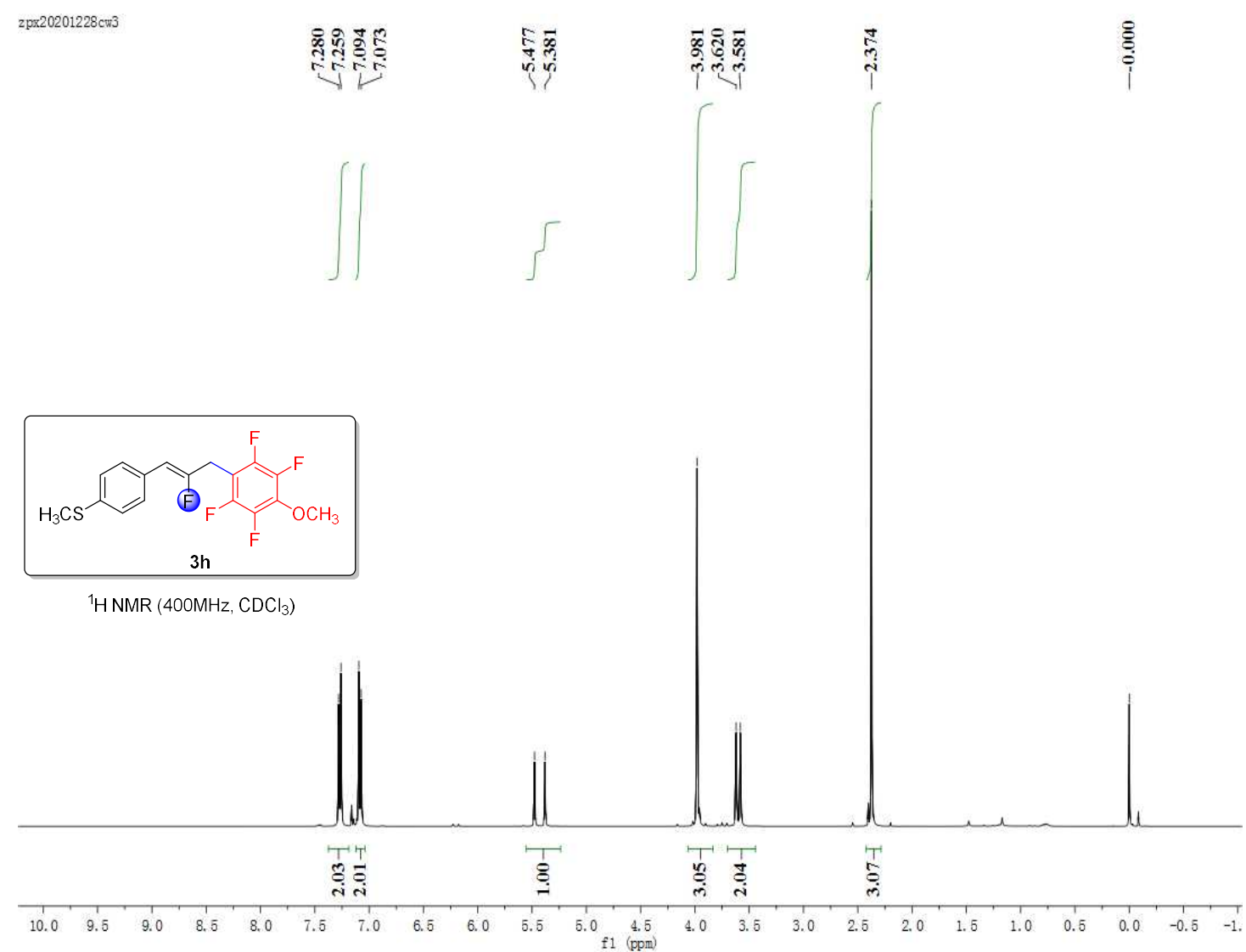

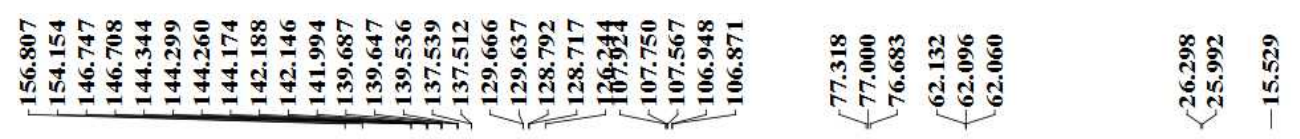

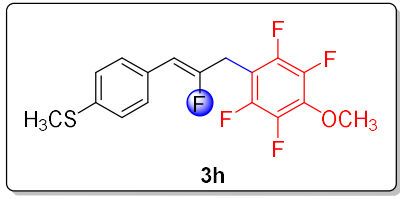

${ }^{13} \mathrm{C} \mathrm{NMR}\left(100 \mathrm{MHz}, \mathrm{CDCl}_{3}\right)$

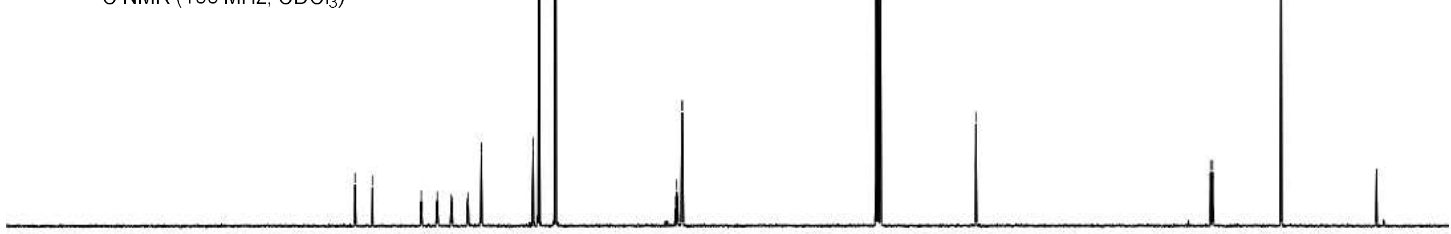

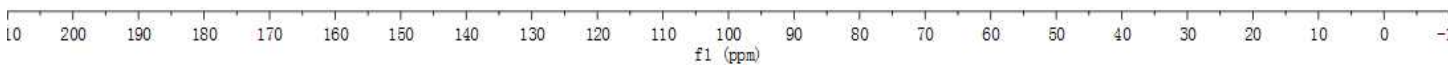



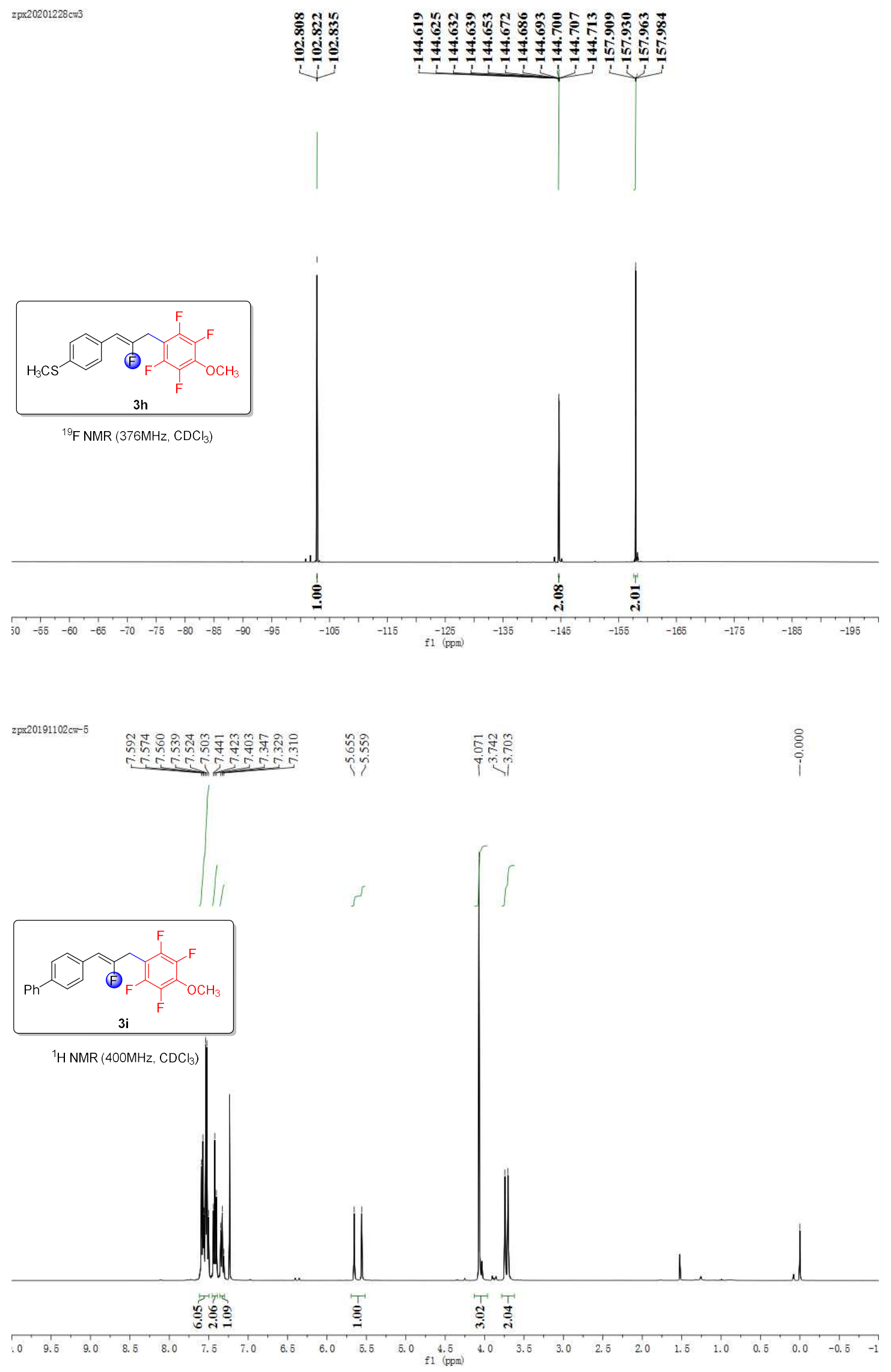


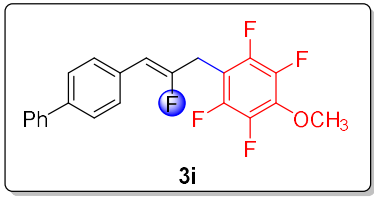

$\left.{ }^{13} \mathrm{C} \mathrm{NMR} \mathrm{(100} \mathrm{MHz,} \mathrm{CDCl} 3\right)$
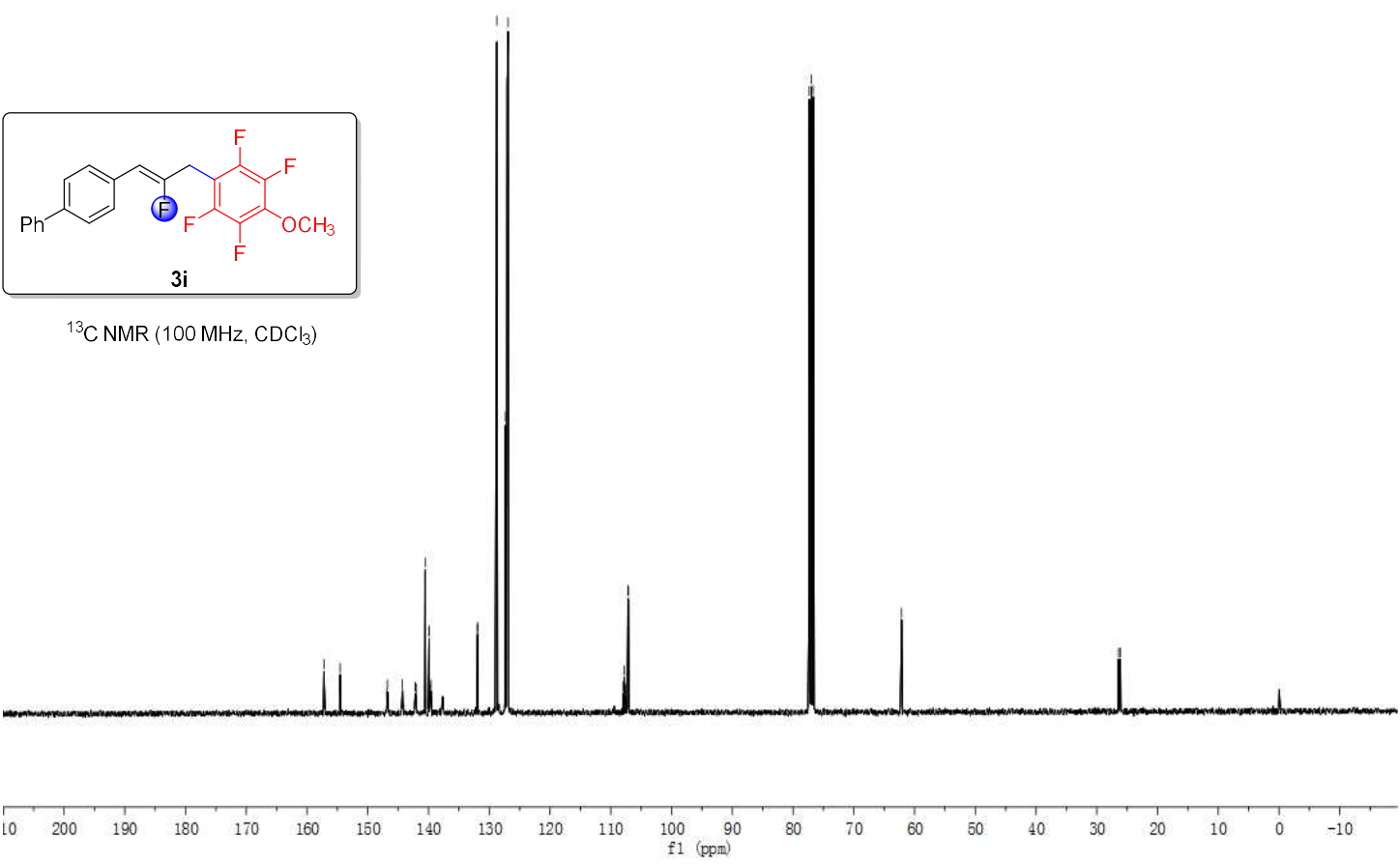

zpx20191102cw-5

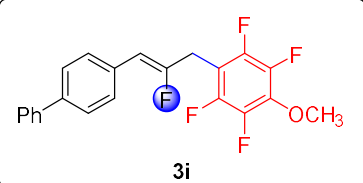

${ }^{19} \mathrm{~F} \mathrm{NMR}\left(376 \mathrm{MHz} \quad \mathrm{CDC}_{3}\right)$

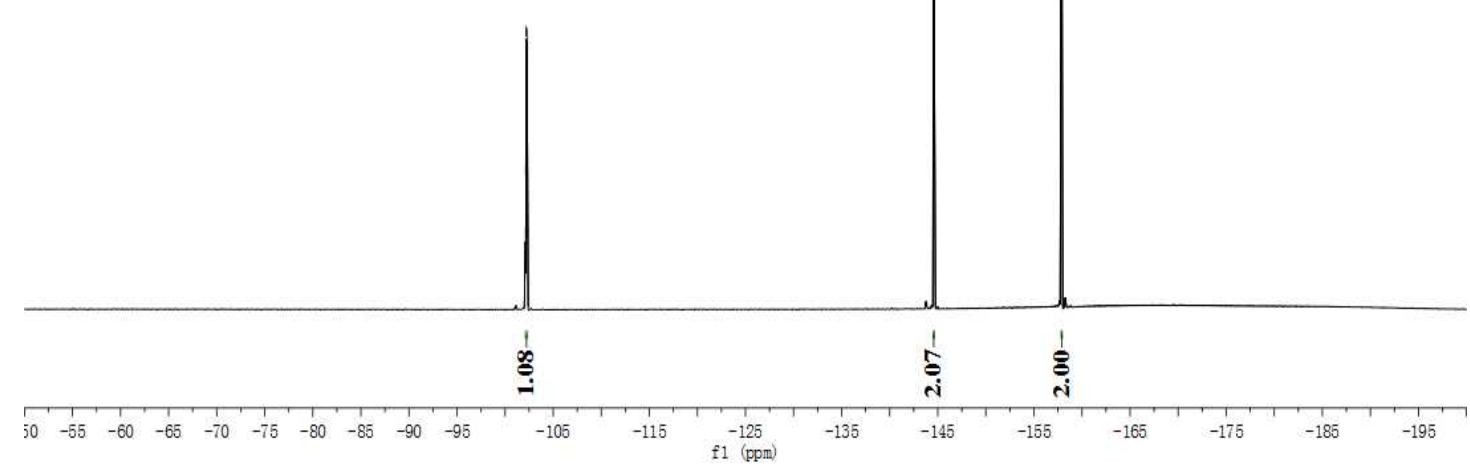




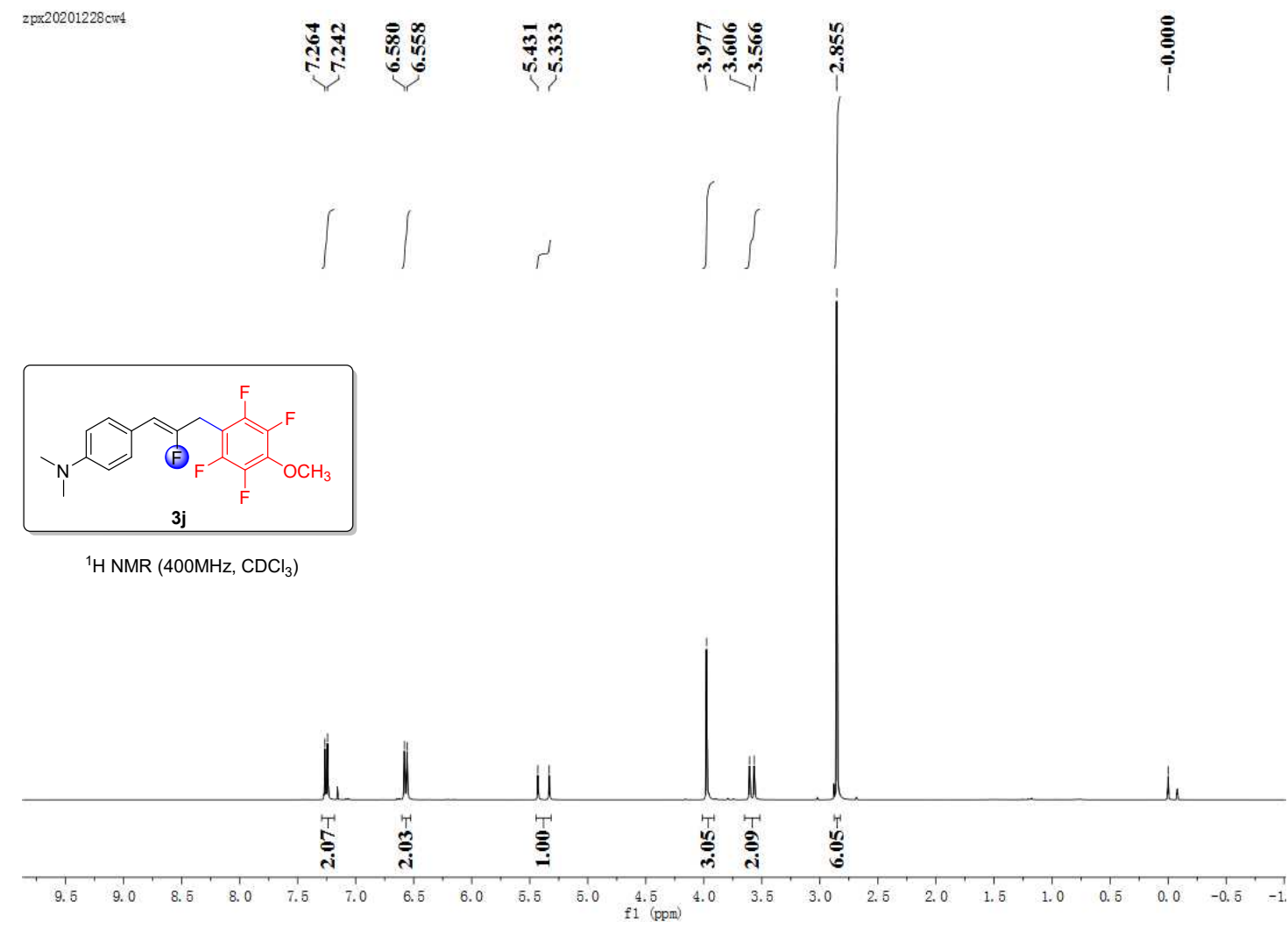

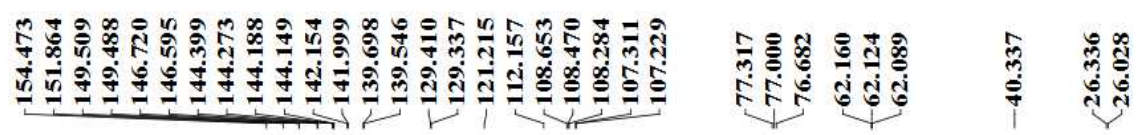

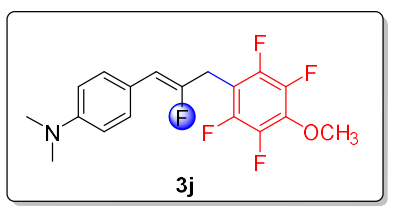

${ }^{13} \mathrm{C} \mathrm{NMR}\left(100 \mathrm{MHz}, \mathrm{CDCl}_{3}\right)$

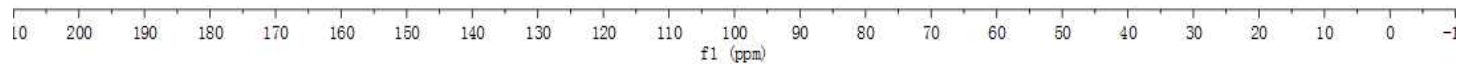




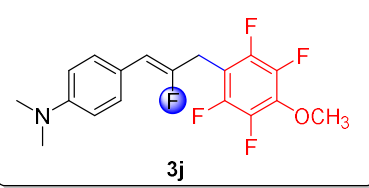

${ }^{1 \mathrm{C}} \mathrm{F} \mathrm{NMR}\left(376 \mathrm{MHz}, \mathrm{CDCl}_{3}\right)$

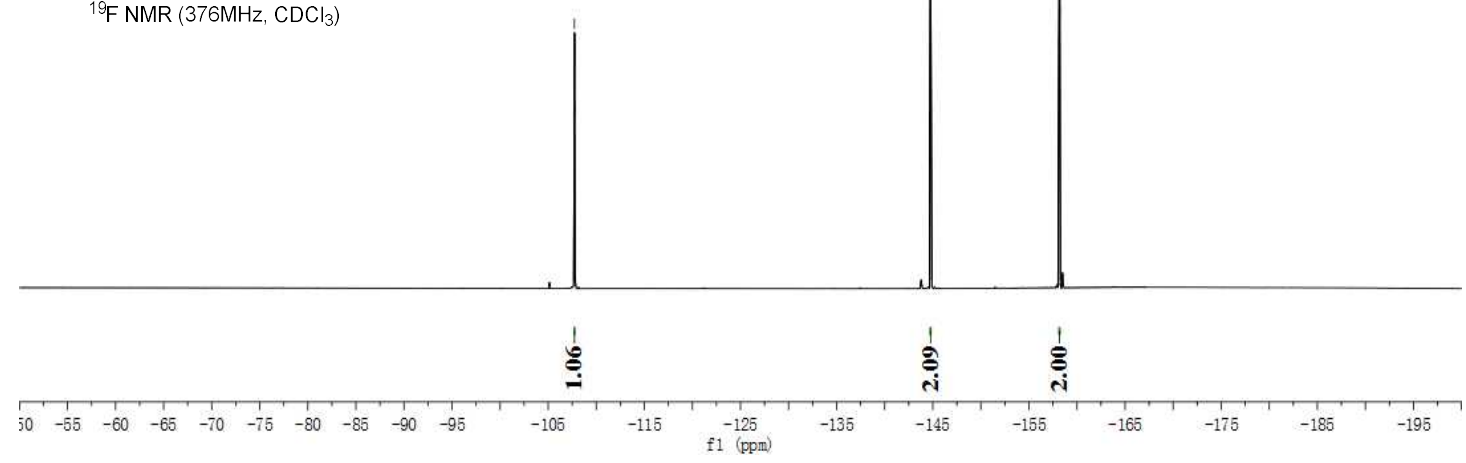

zpx20191102cw-12

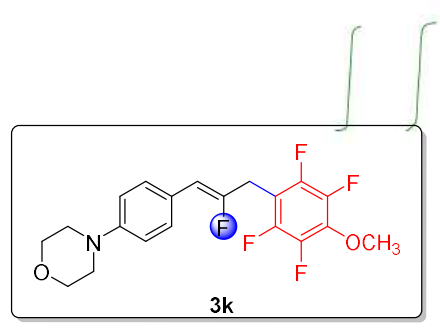

${ }^{1} \mathrm{H} \mathrm{NMR}\left(400 \mathrm{MHz} \mathrm{CDCl}_{3}\right)$
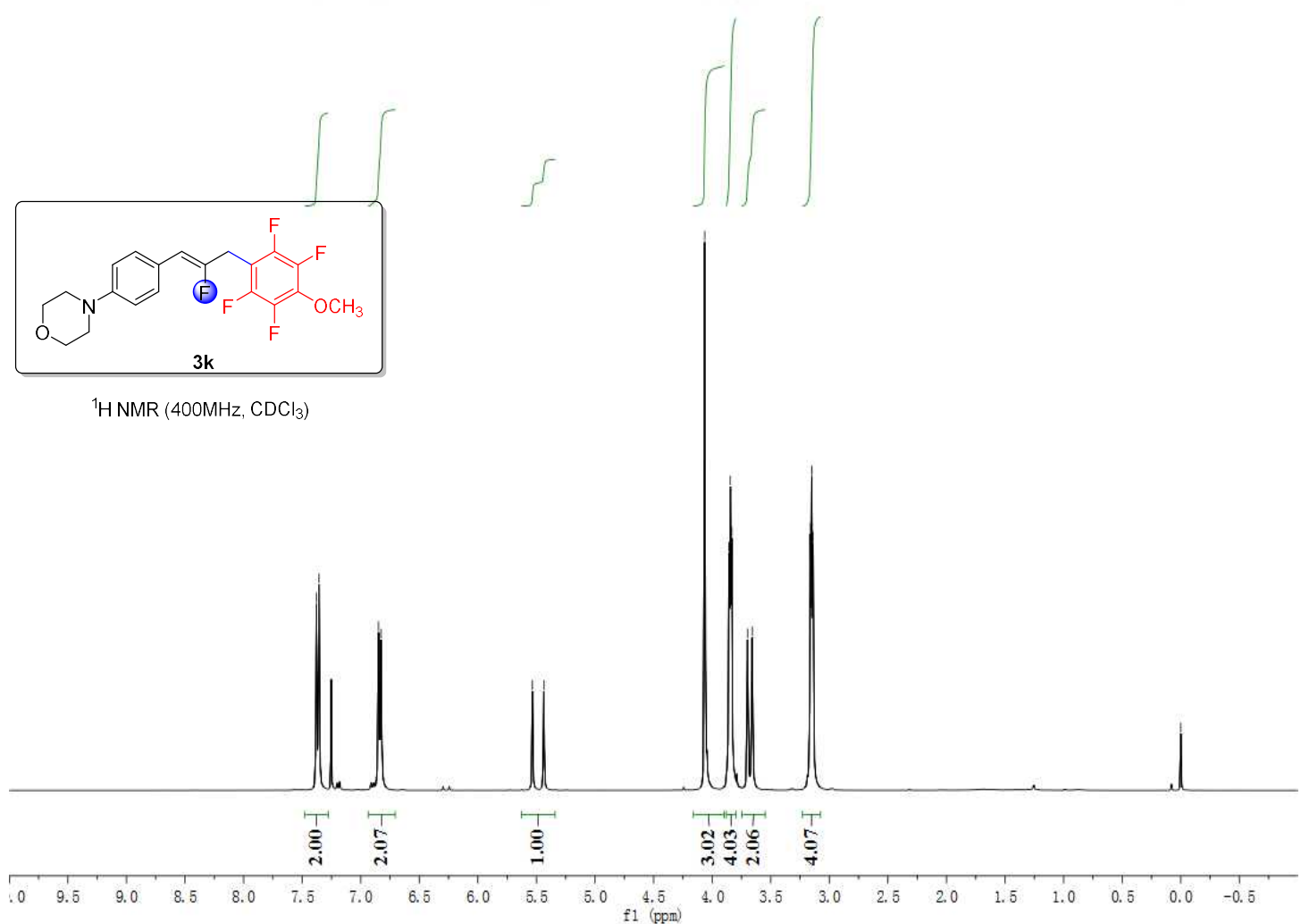


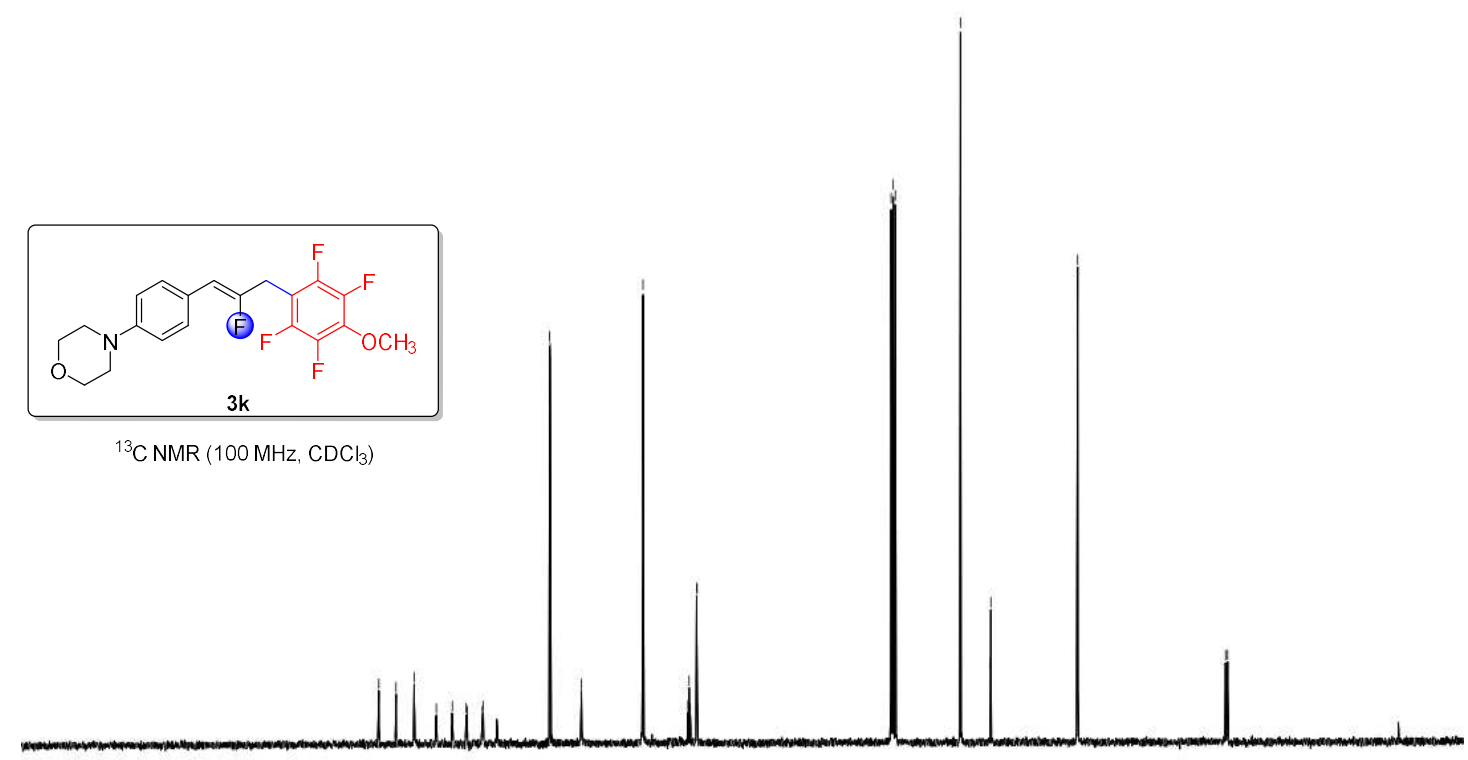

zpx20191102cw-12

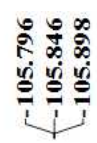

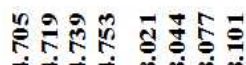

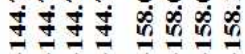

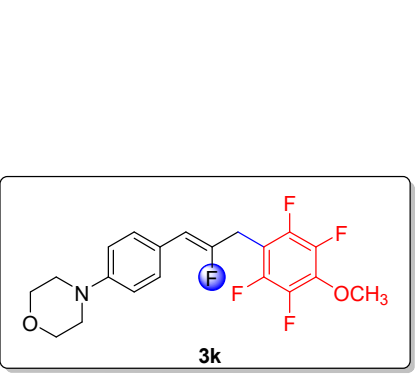

${ }^{19} \mathrm{~F}$ NMR $\left(376 \mathrm{MHz}, \mathrm{CDCl}_{3}\right)$
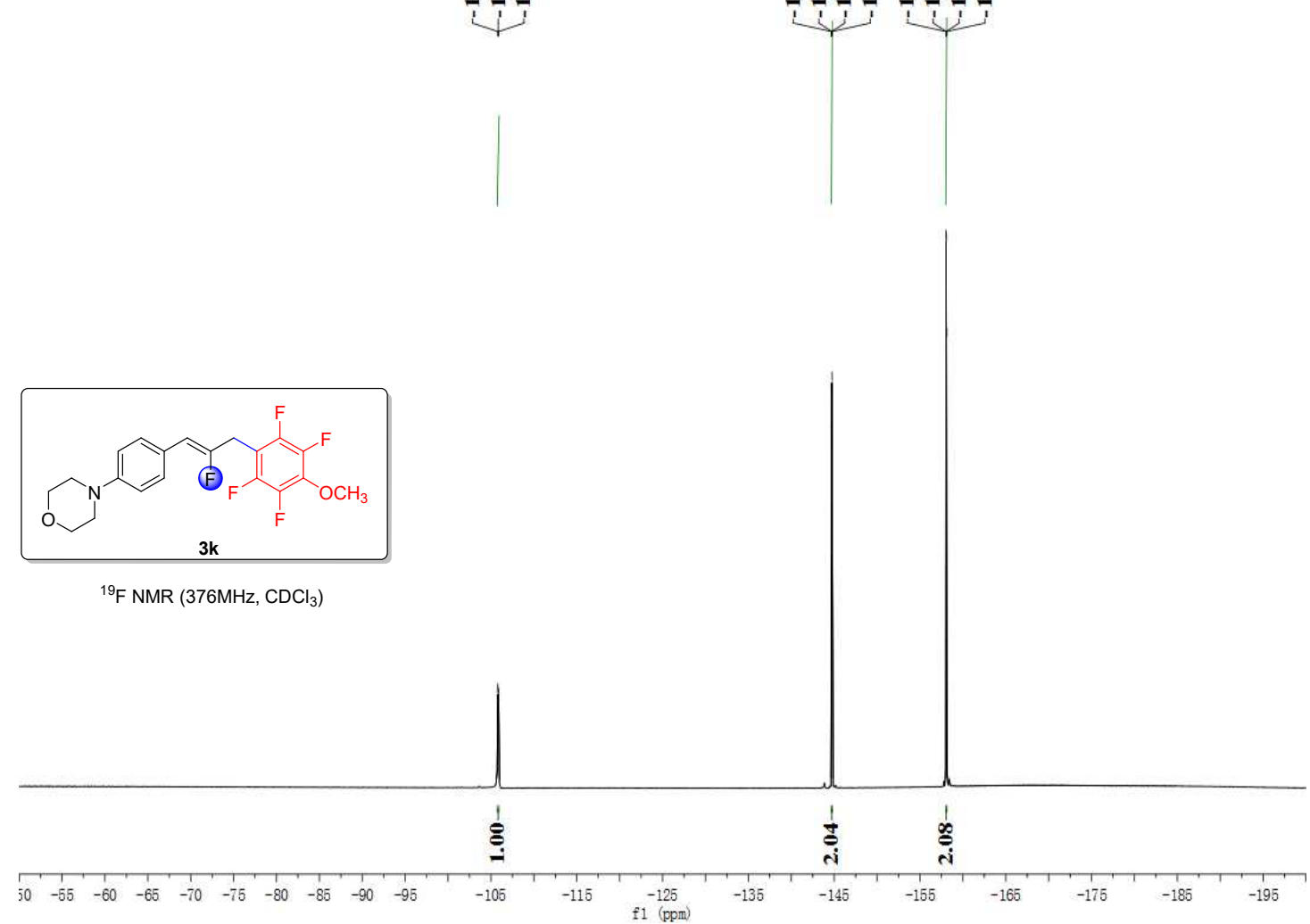

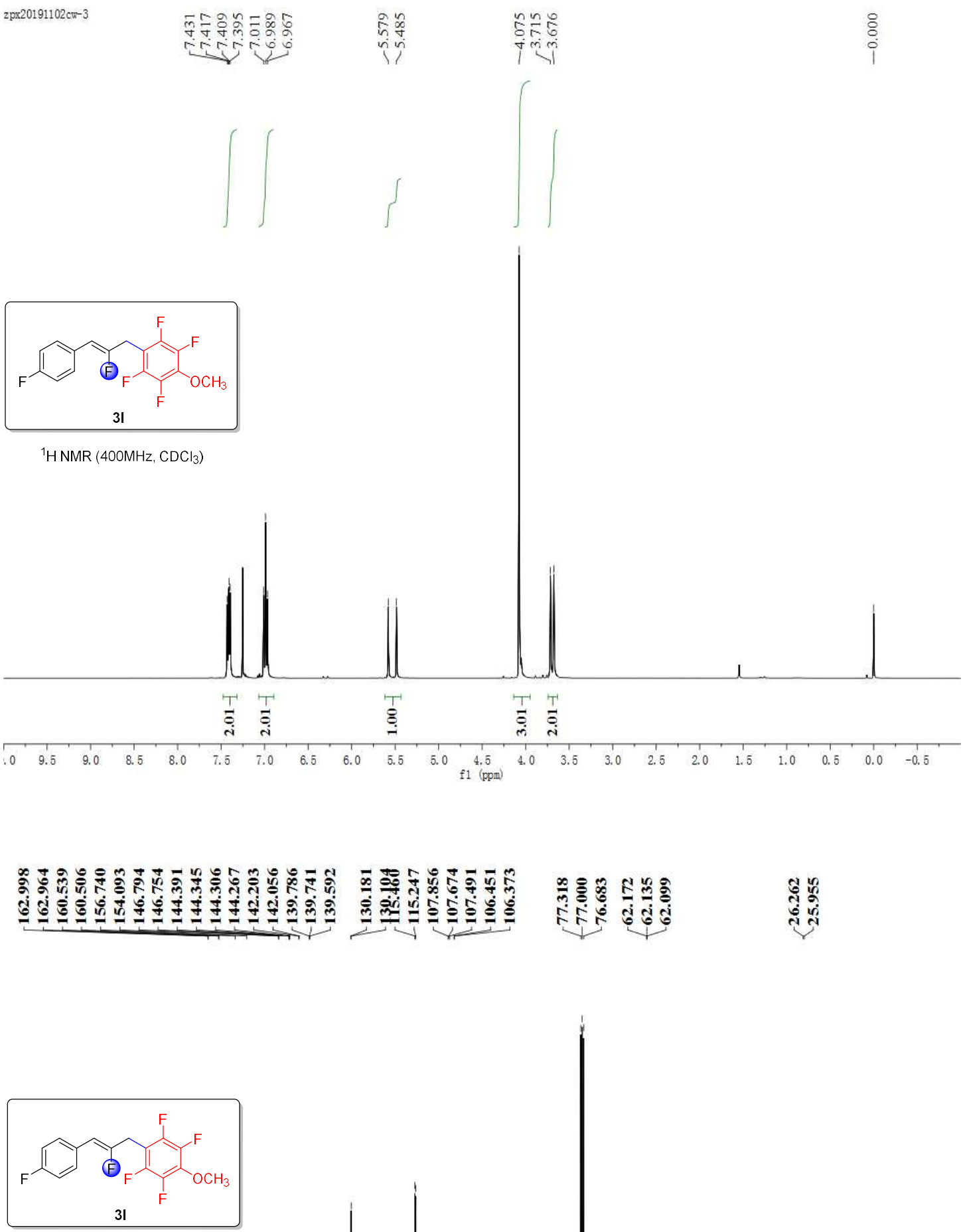

${ }^{13} \mathrm{CNMR}\left(100 \mathrm{MHz}, \mathrm{CDCl}_{3}\right)$
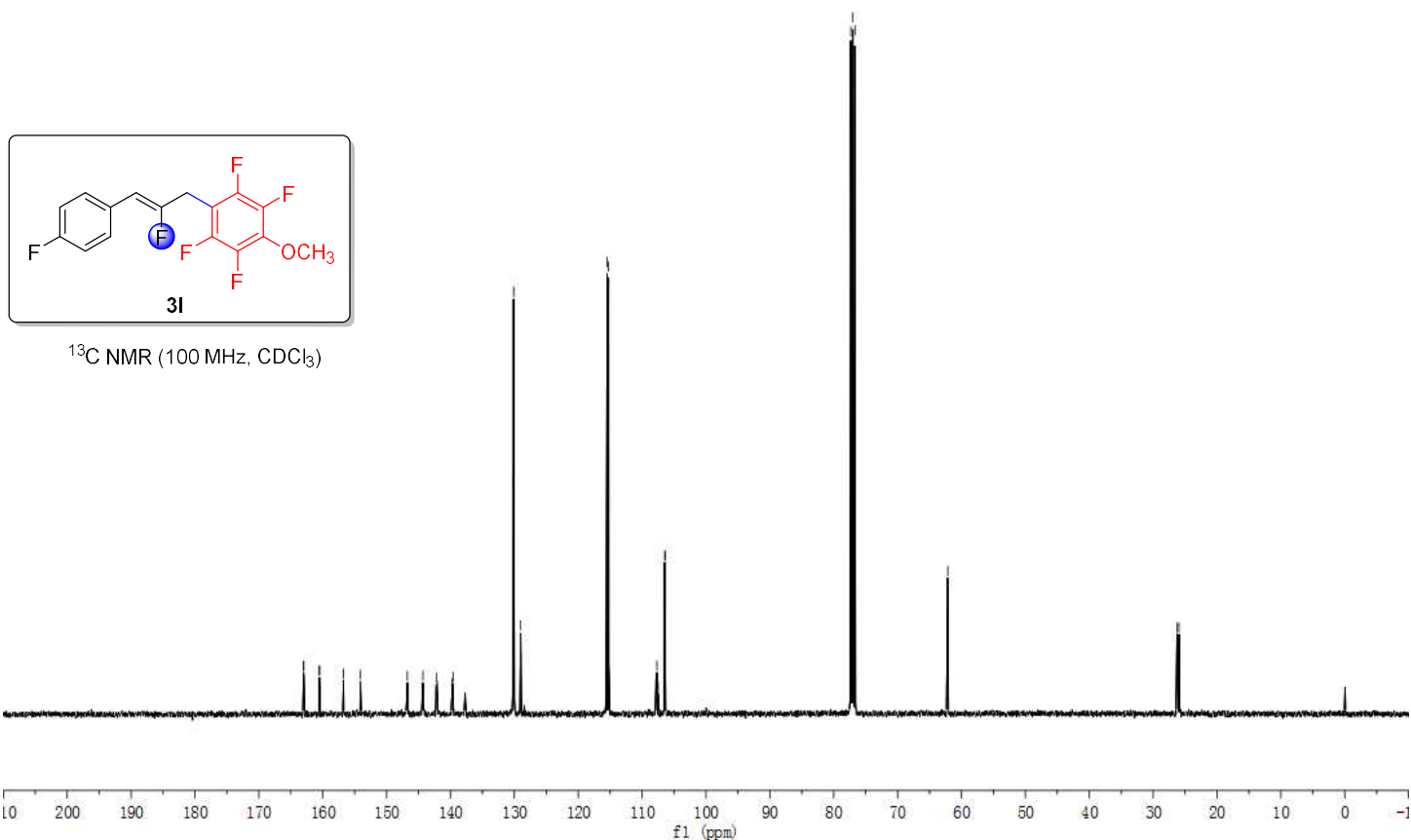

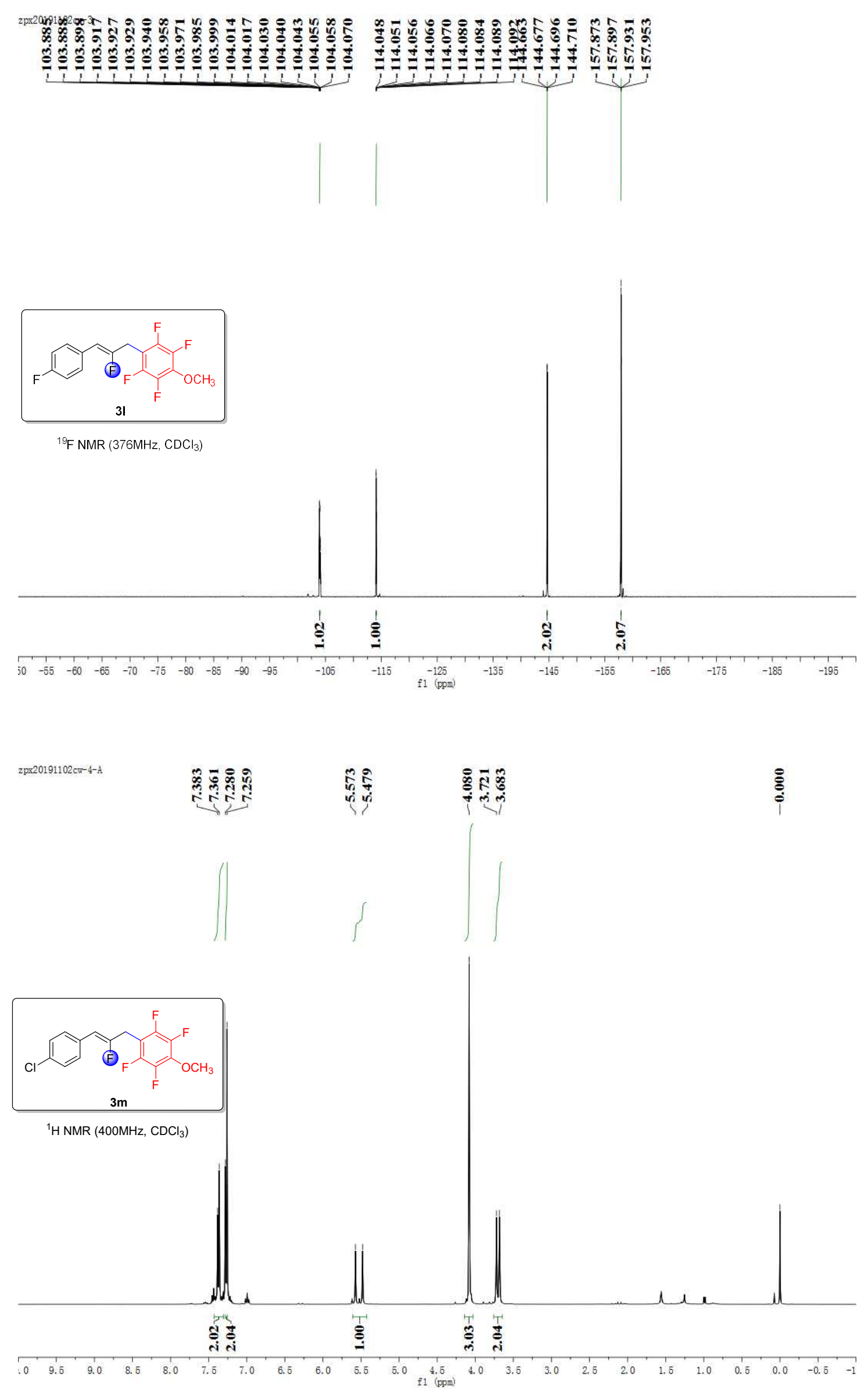


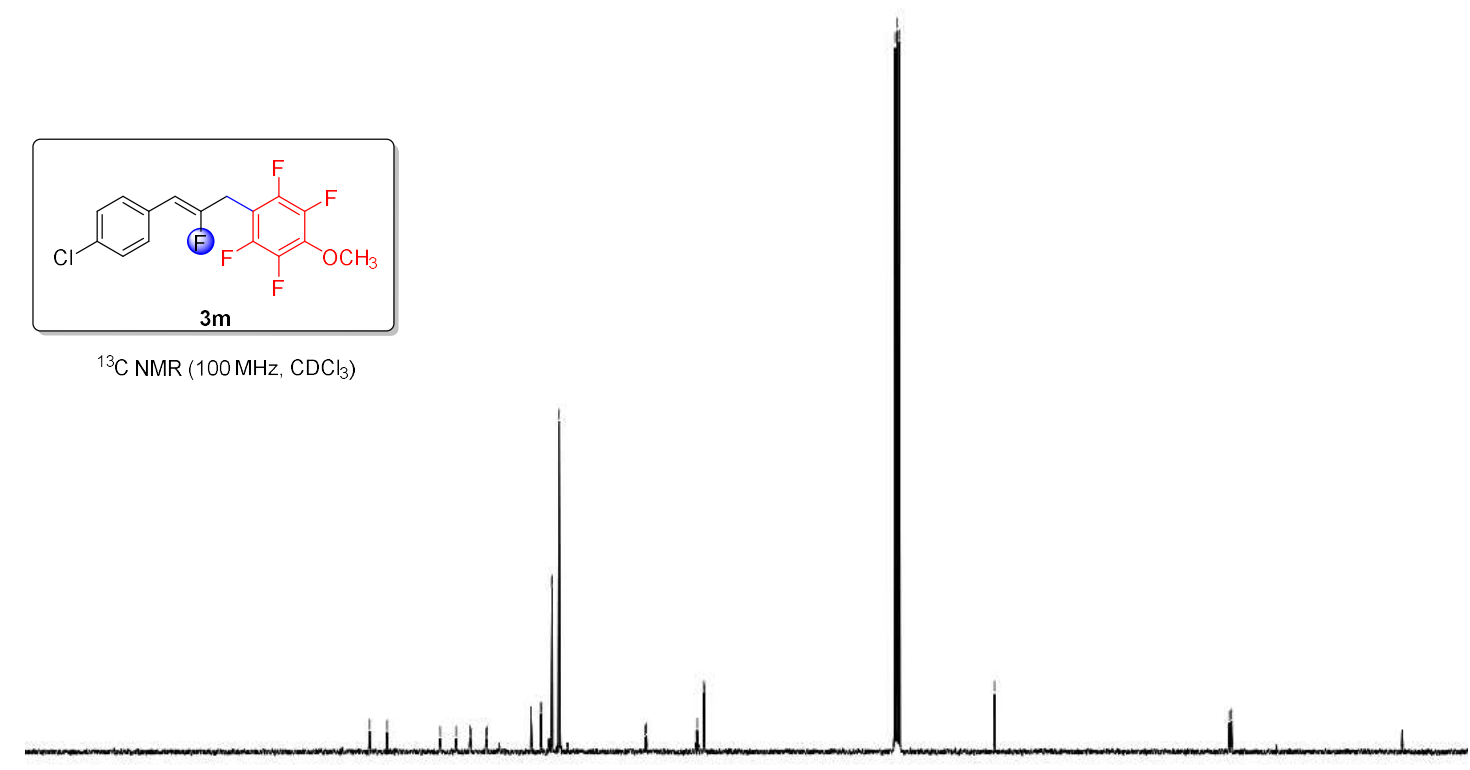

$\left.{ }^{13} \mathrm{C} \mathrm{NMR} \mathrm{(100} \mathrm{MHz} \mathrm{CDC}_{3}\right)$

zpx20191102 $\mathrm{cm}-4-\mathrm{A}$

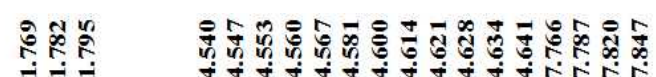

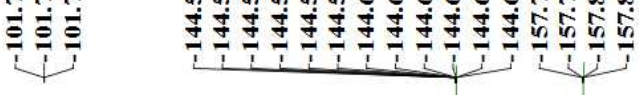
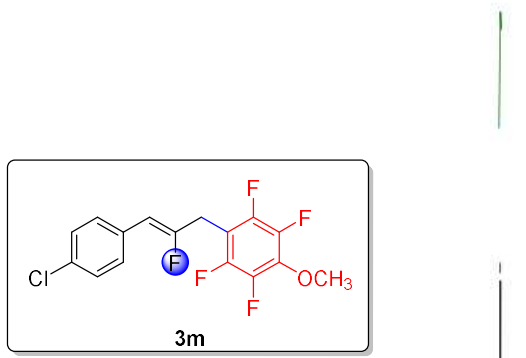

${ }^{19} \mathrm{~F} \mathrm{NMR}\left(376 \mathrm{MHz}, \mathrm{CDCl}_{3}\right)$

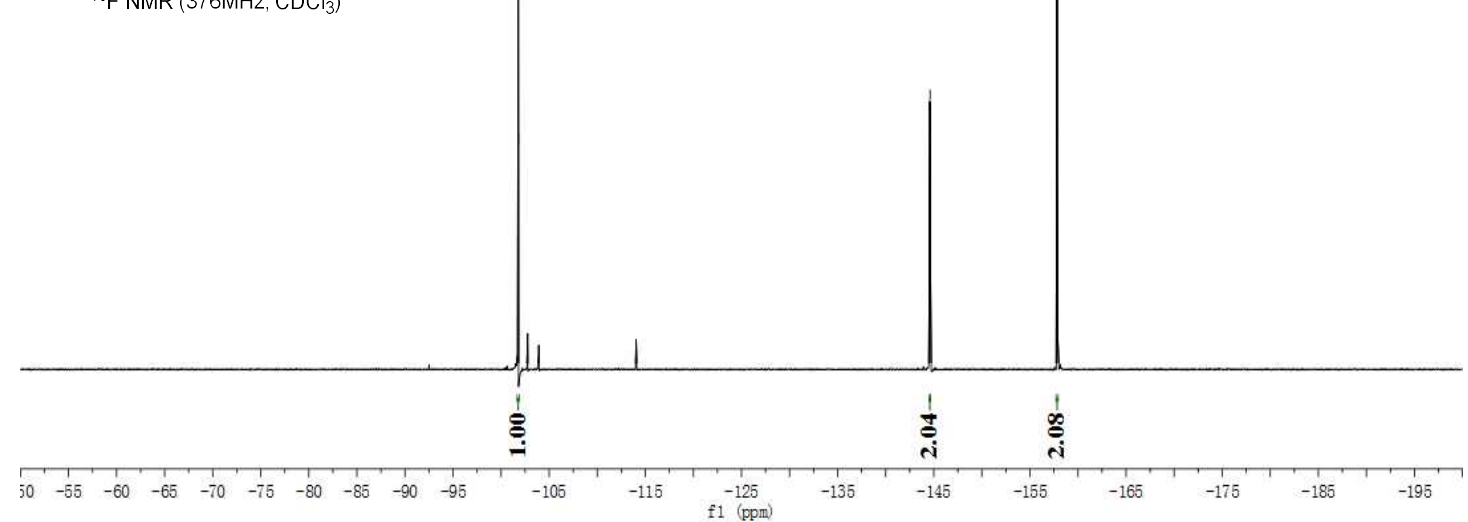



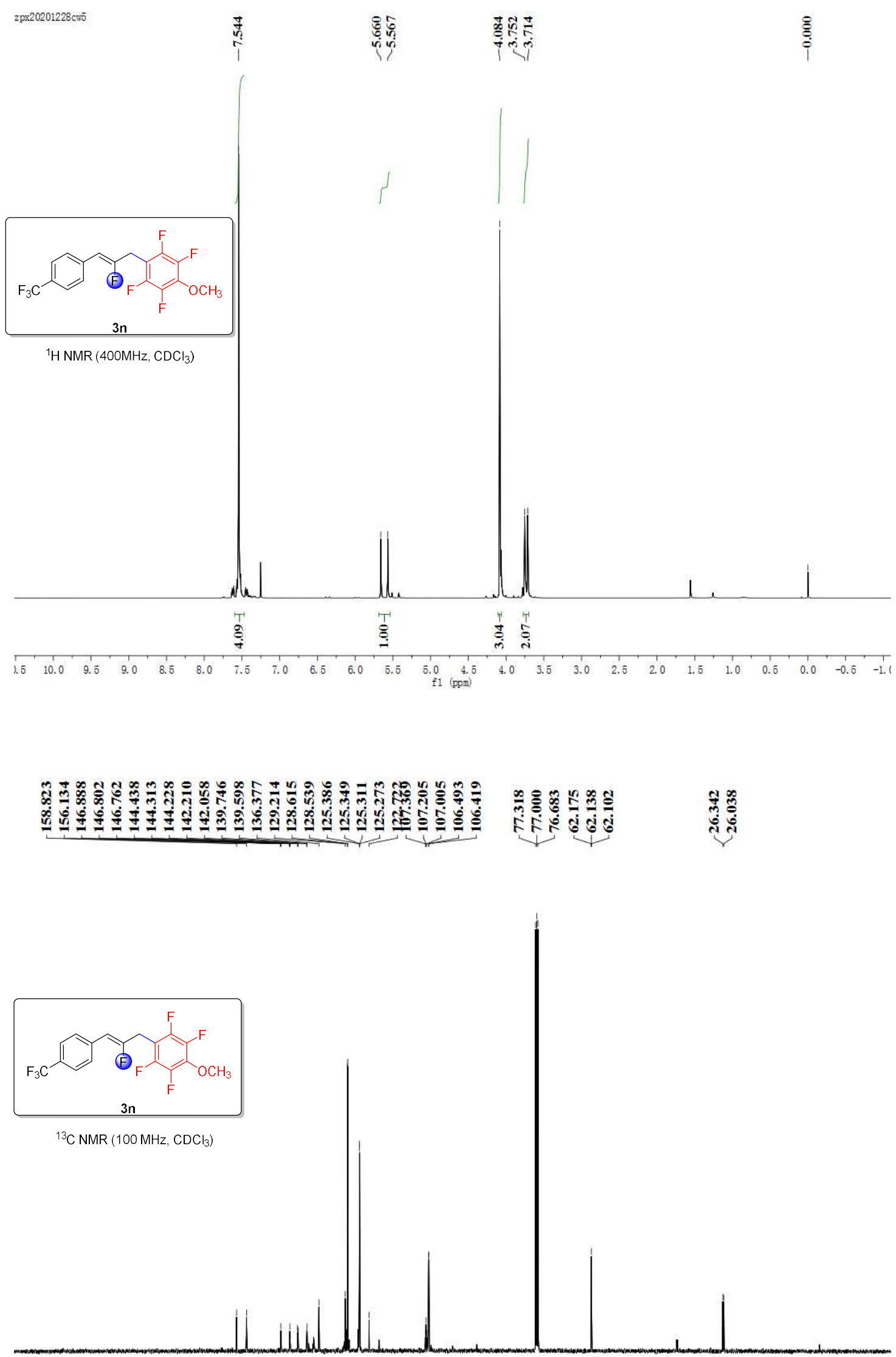

${ }^{13} \mathrm{C} \mathrm{NMR}\left(100 \mathrm{MHz}, \mathrm{CDCl}_{3}\right)$

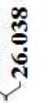

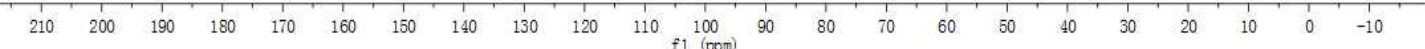



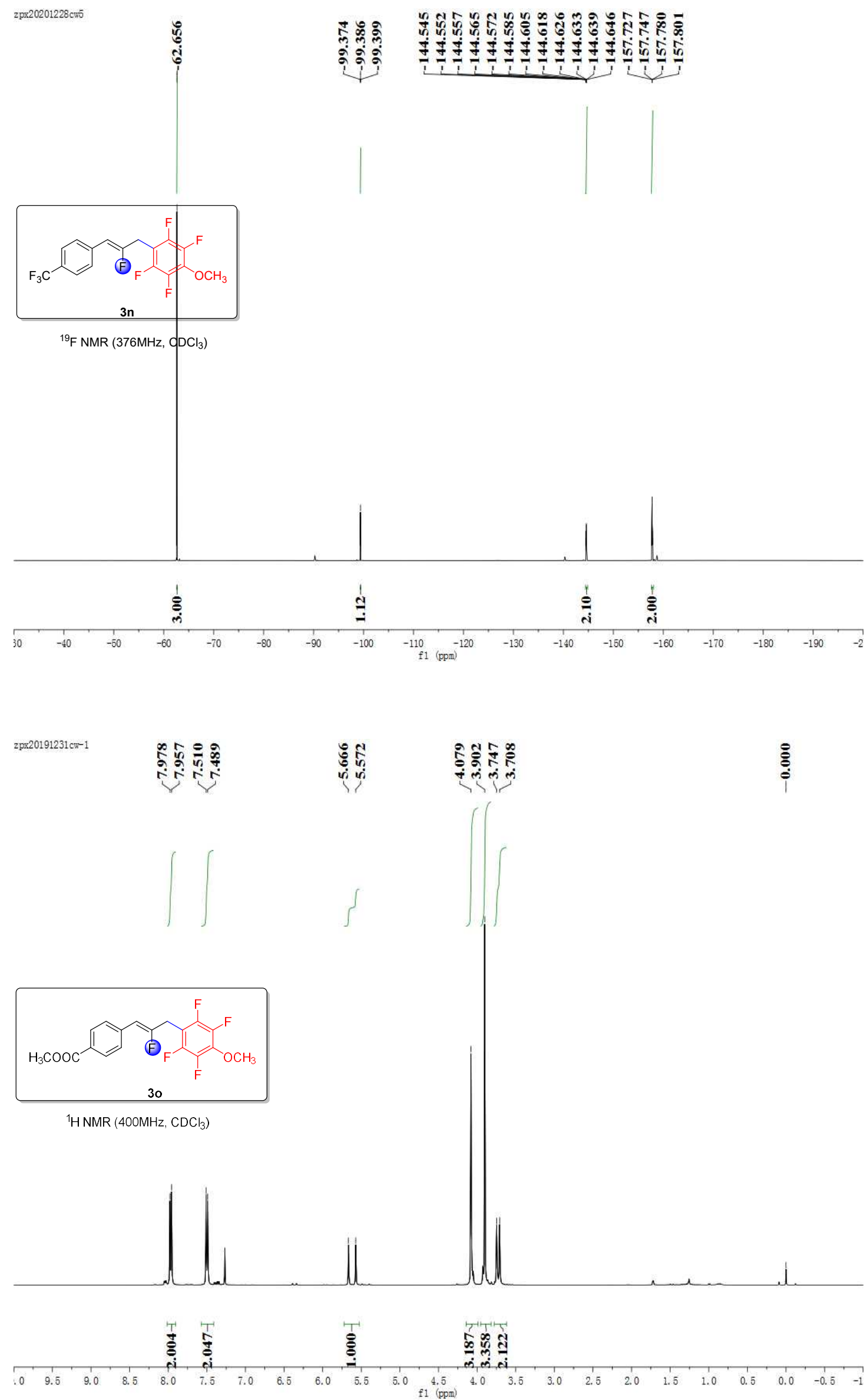


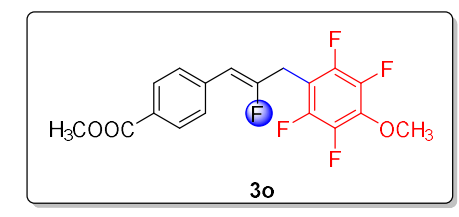

${ }^{13} \mathrm{CNMR}\left(100 \mathrm{MHz},\left.\mathrm{CDC}\right|_{3}\right)$

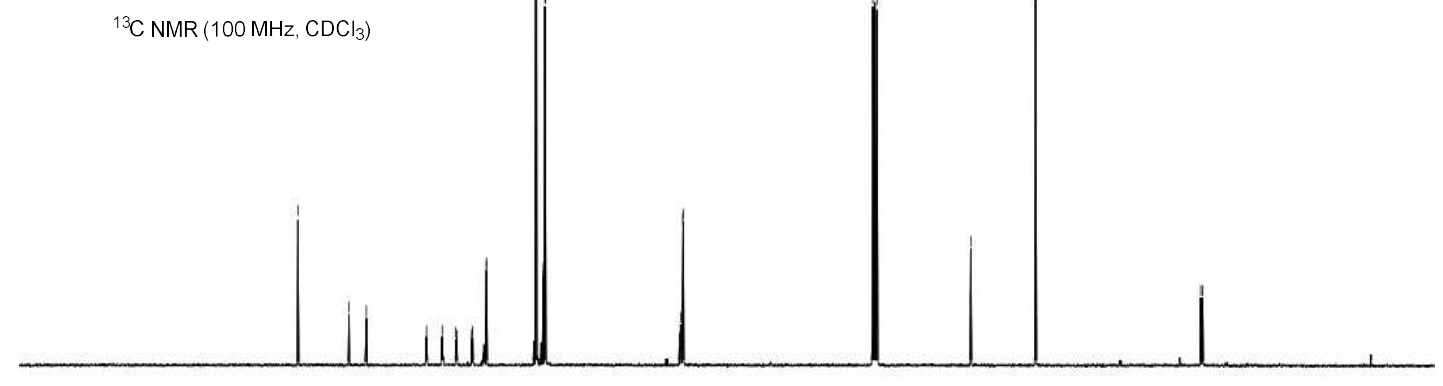

\begin{tabular}{llllllllllllllllllllllllllll}
\hline 0 & 200 & 190 & 180 & 170 & 160 & 150 & 140 & 130 & 120 & 110 & 100 & 90 & 80 & 70 & 60 & 50 & 40 & 30 & 20 & 10 & 0 & -1
\end{tabular}

zpx20191231 cw-1

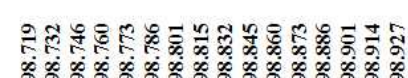

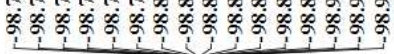
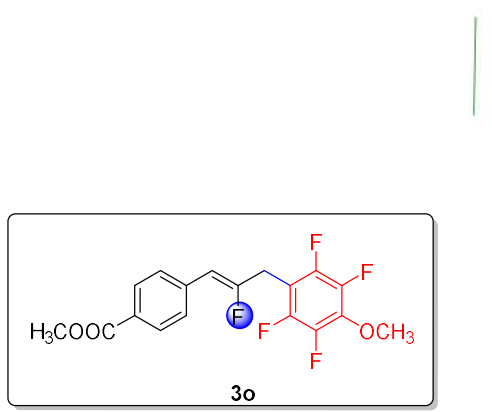

${ }^{1 \mathrm{C}} \mathrm{F} \mathrm{NMR}\left(376 \mathrm{MHz} \quad \mathrm{CDCl}_{3}\right)$

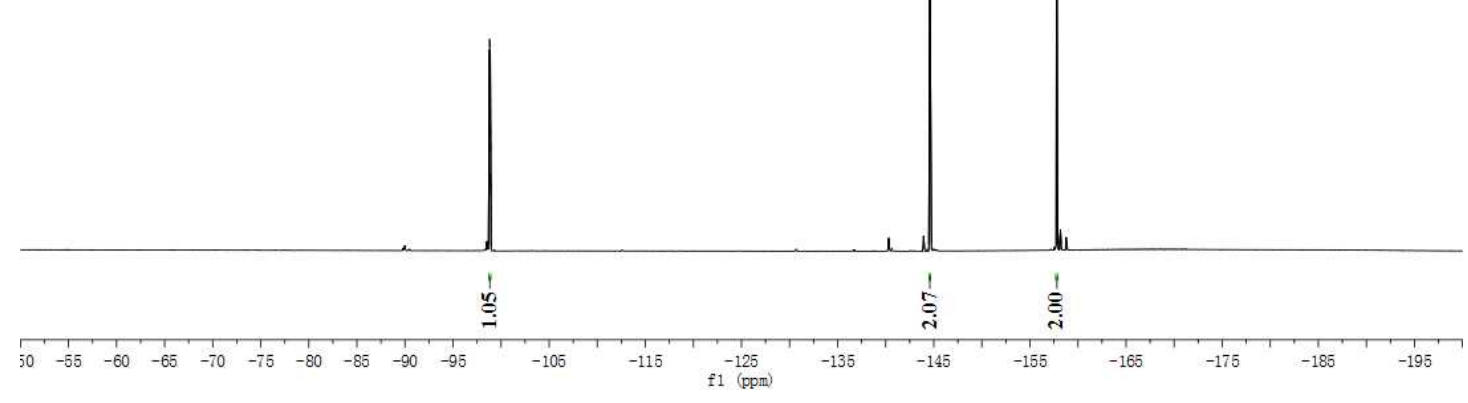




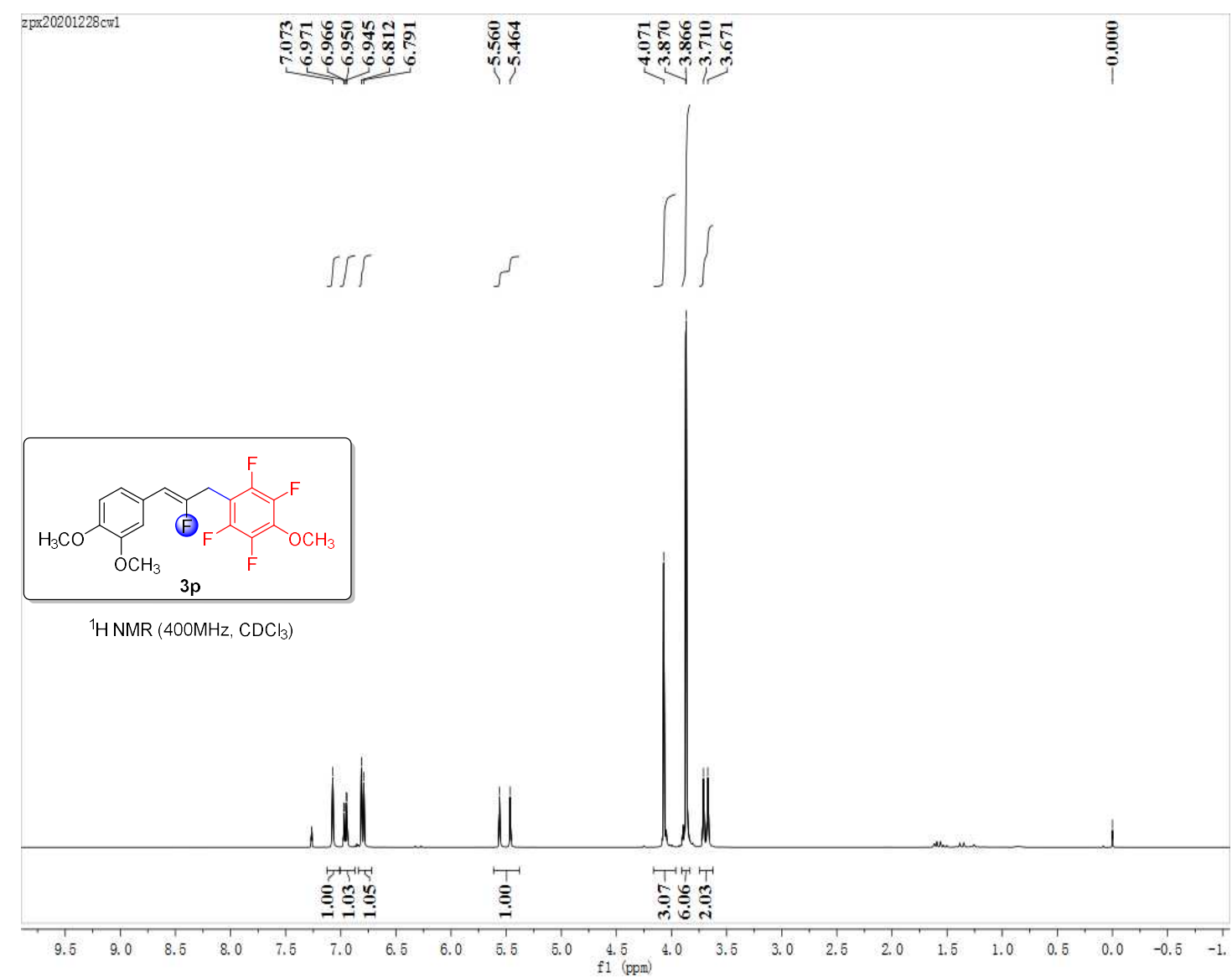

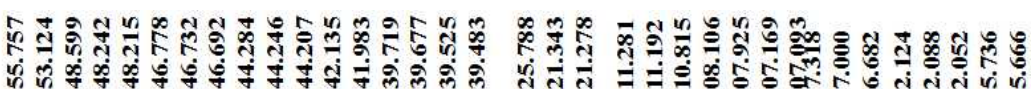

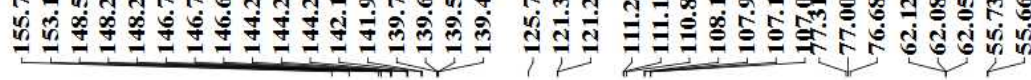

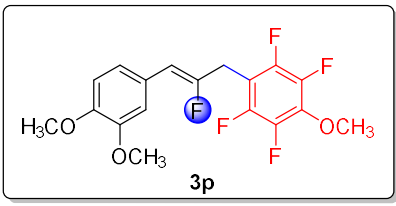

${ }^{13} \mathrm{CNMR}\left(100 \mathrm{MHz}, \mathrm{CDCl}_{3}\right)$
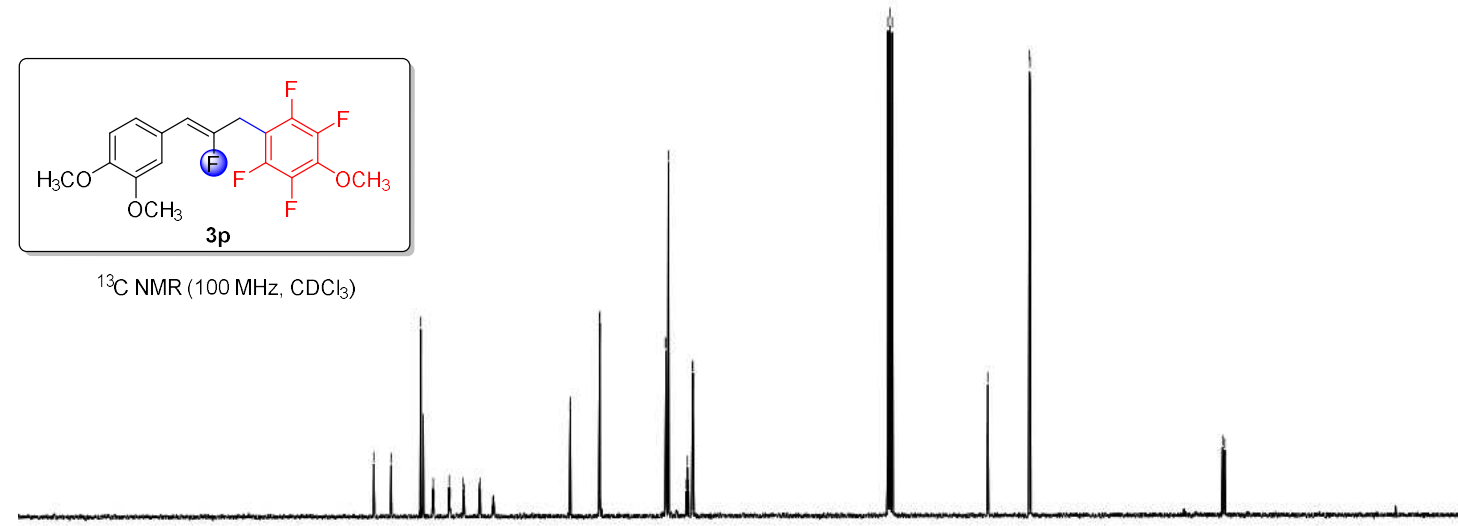

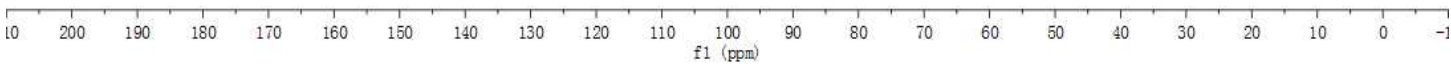




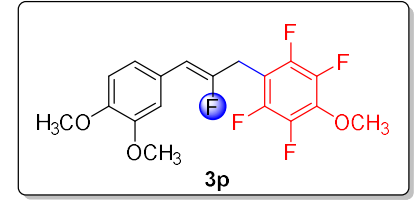

${ }^{10} \mathrm{~F} \mathrm{NMR}\left(376 \mathrm{MHz}, \mathrm{CDCl}_{3}\right)$

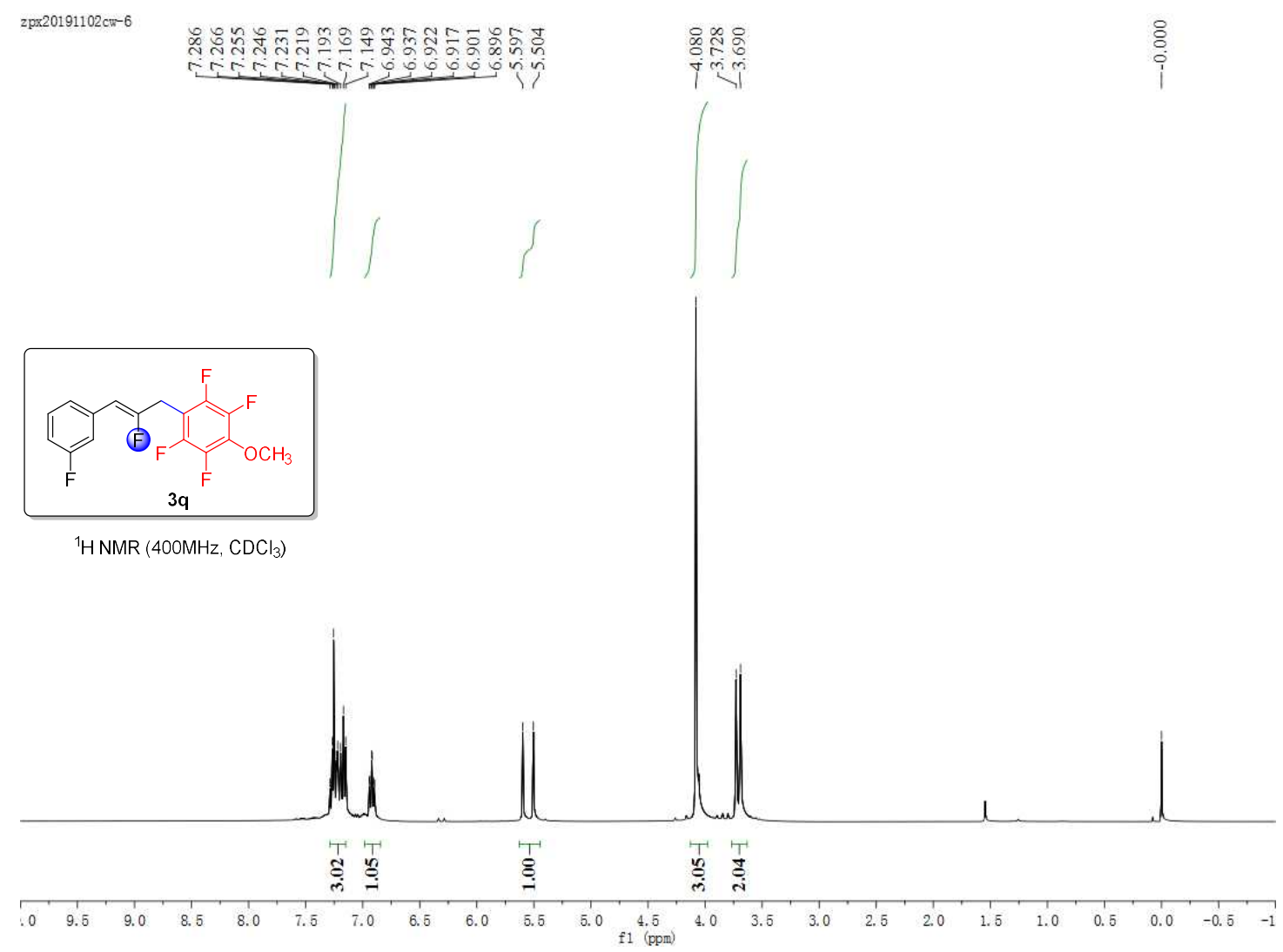




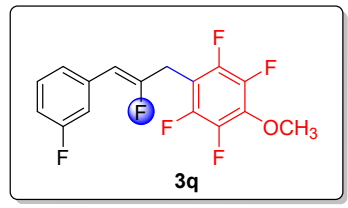

$\left.{ }^{13} \mathrm{C} \mathrm{NMR} \mathrm{(100} \mathrm{MHz,} \mathrm{CDCl}_{3}\right)$
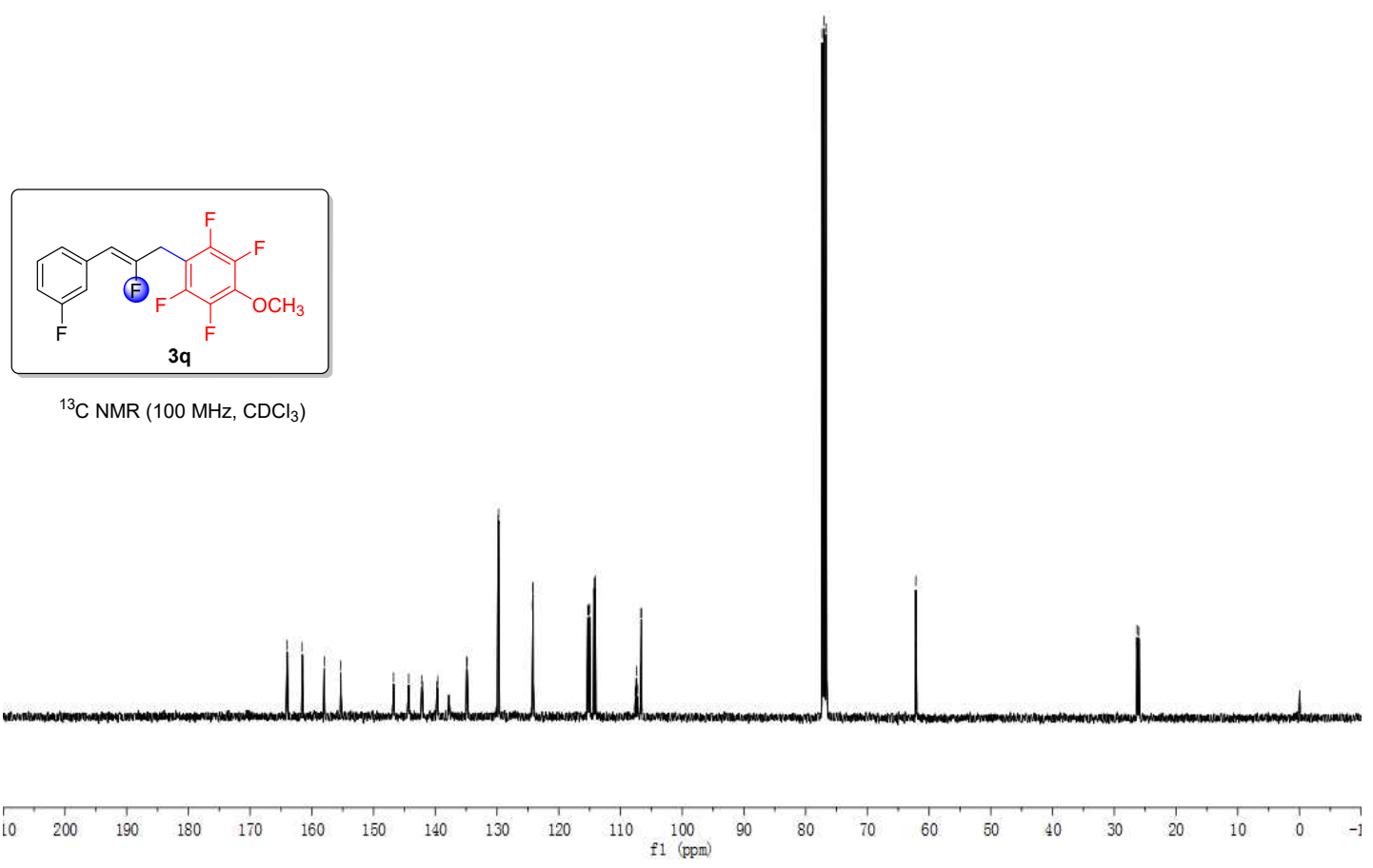

\section{2px20191102-

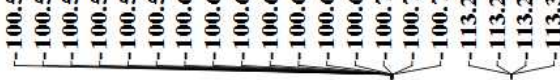

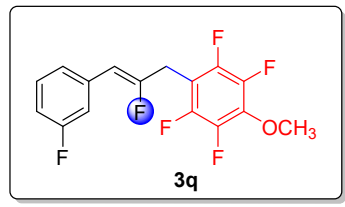

${ }^{19} \mathrm{~F}$ NMR $\left(376 \mathrm{MHz}, \mathrm{CDCl}_{3}\right)$
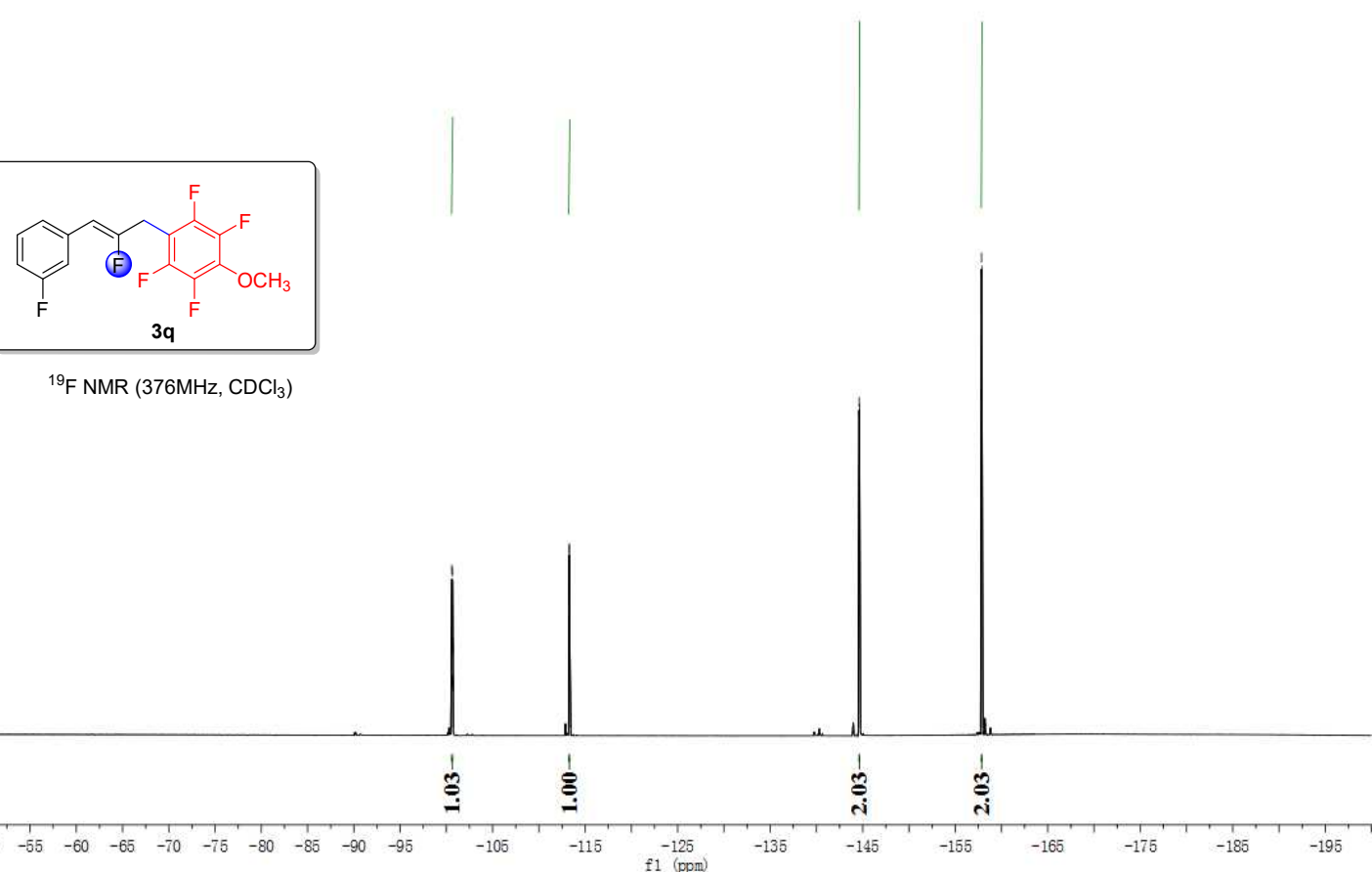


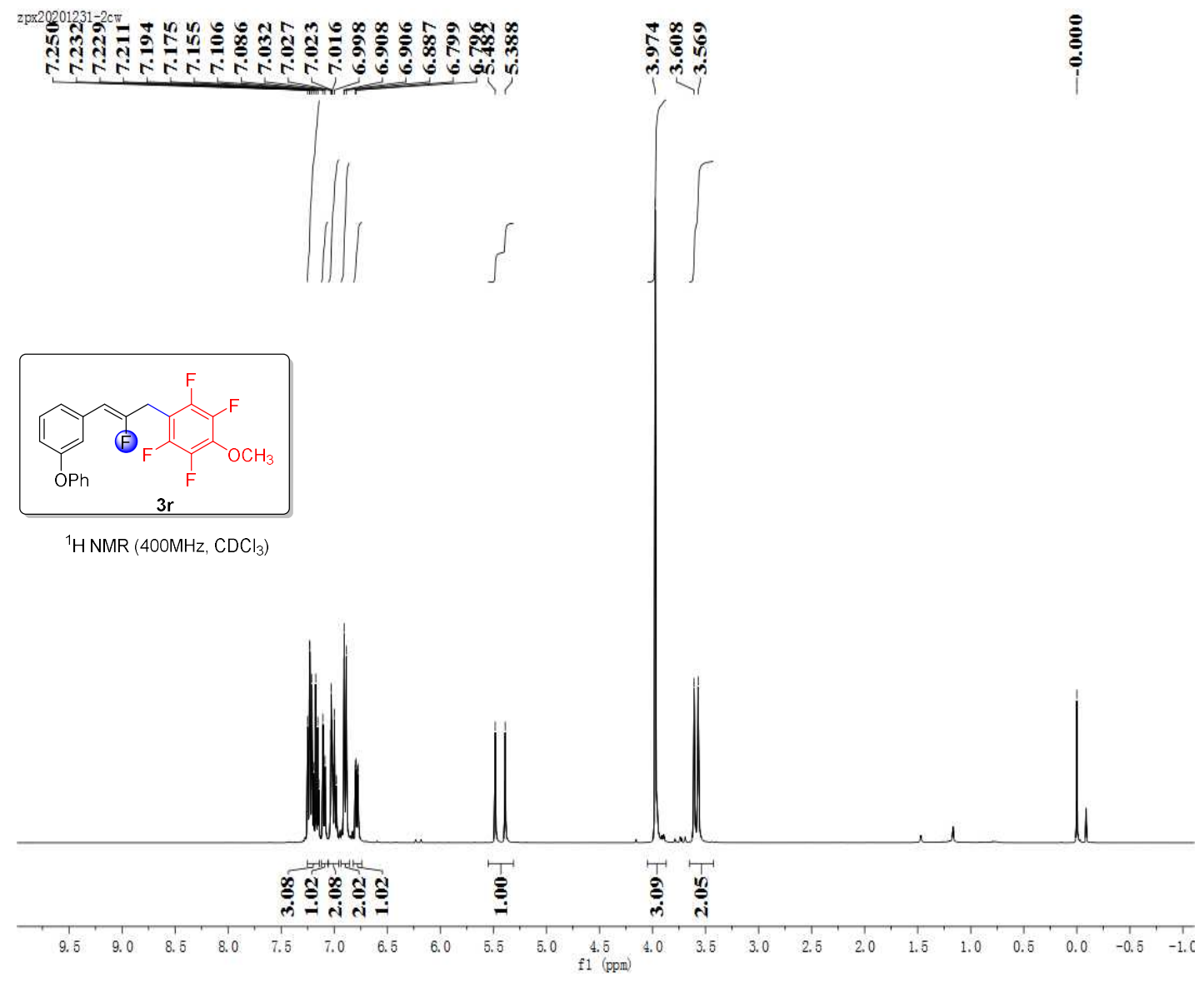

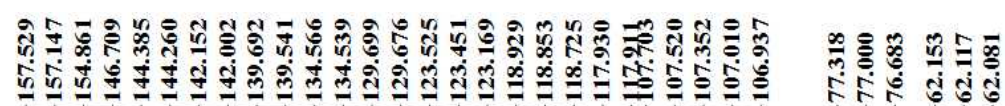

तู

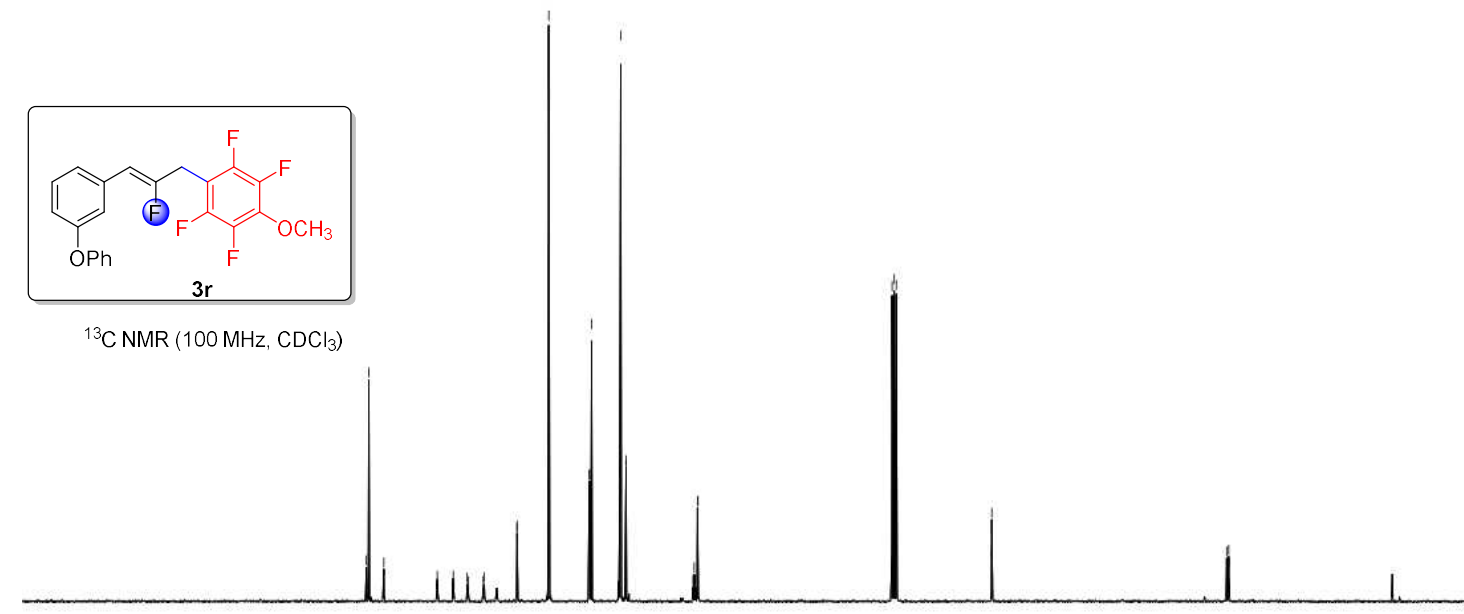

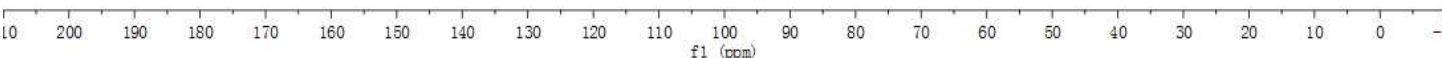


zpx20201231-2cw

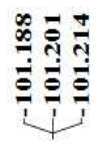

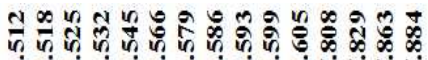

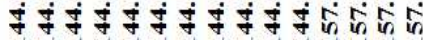

i

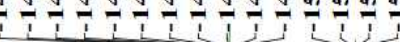

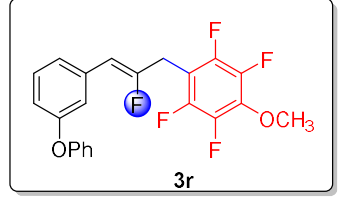

${ }^{1 \subseteq} \mathrm{F} \mathrm{NMR}\left(376 \mathrm{MHz}, \mathrm{CDCl}_{3}\right)$
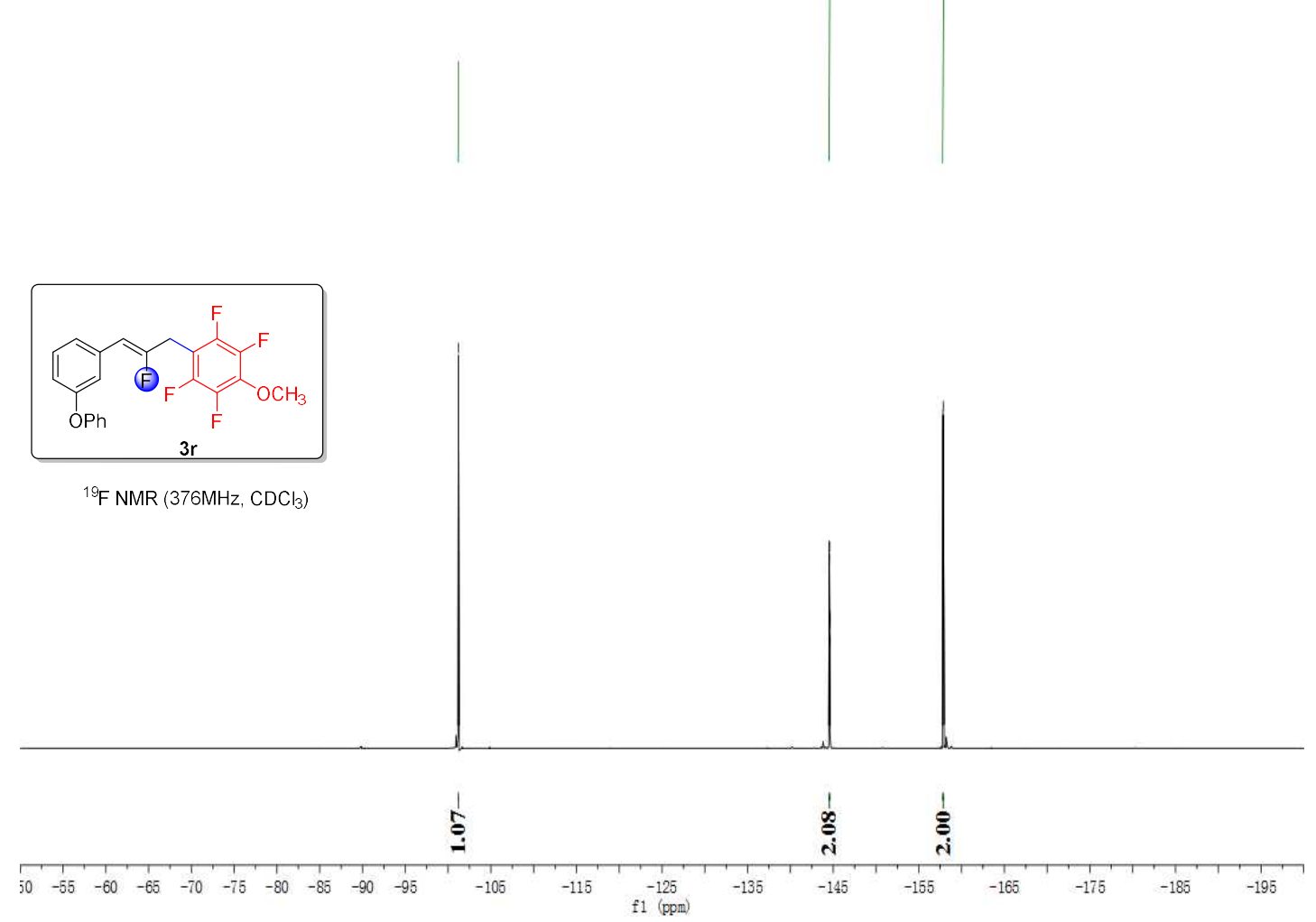

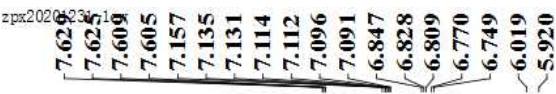

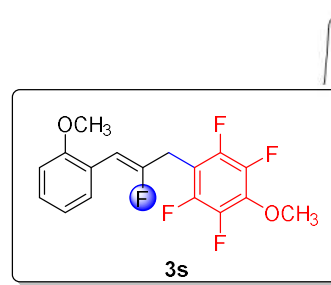

${ }^{1} \mathrm{H}$ NMR $\left(400 \mathrm{MHz}, \mathrm{CDC}_{3}\right)$
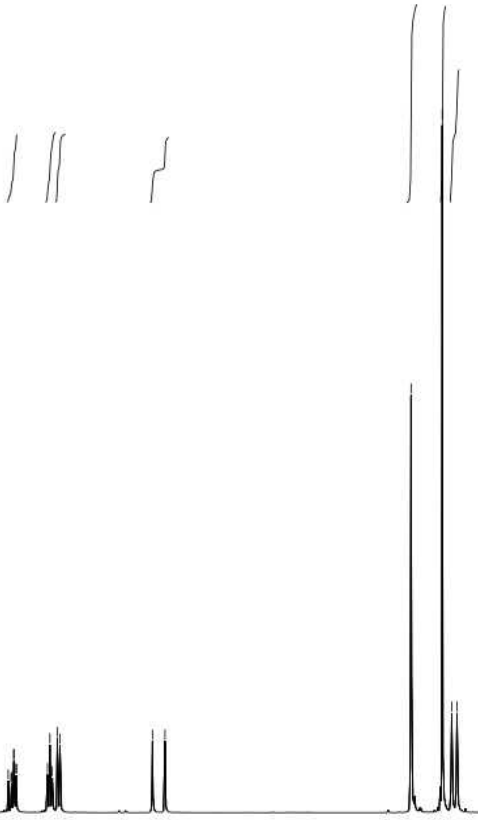

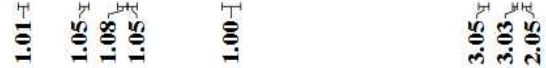

$\begin{array}{llllllllllllllllllllllll}10.0 & 9.5 & 9.0 & 8.5 & 8.0 & 7.5 & 7.0 & 6.5 & 6.0 & 5.5 & 5.0 & \begin{array}{l}1 \\ \text { f.5 }\end{array} & 4.0 & 3.5 & 3.0 & 2.5 & 2.0 & 1.5 & 1.0 & 0.5 & 0.0 & -0.5 & -1 .\end{array}$ 


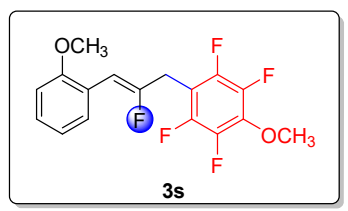

${ }^{13} \mathrm{C} \mathrm{NMR} \mathrm{(100} \mathrm{MHz,} \mathrm{CDCl}_{3}$ )
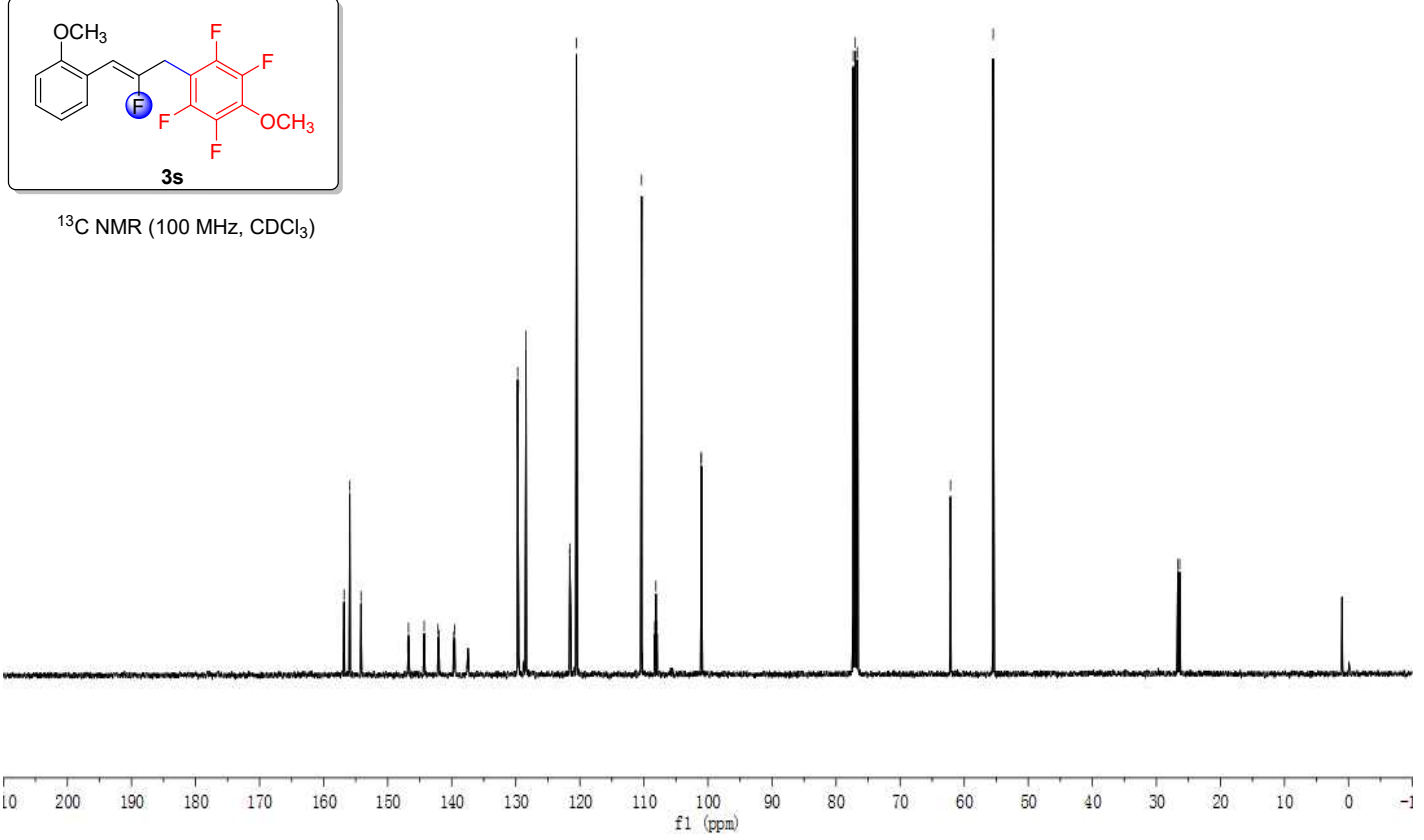

zps20201231-1ew
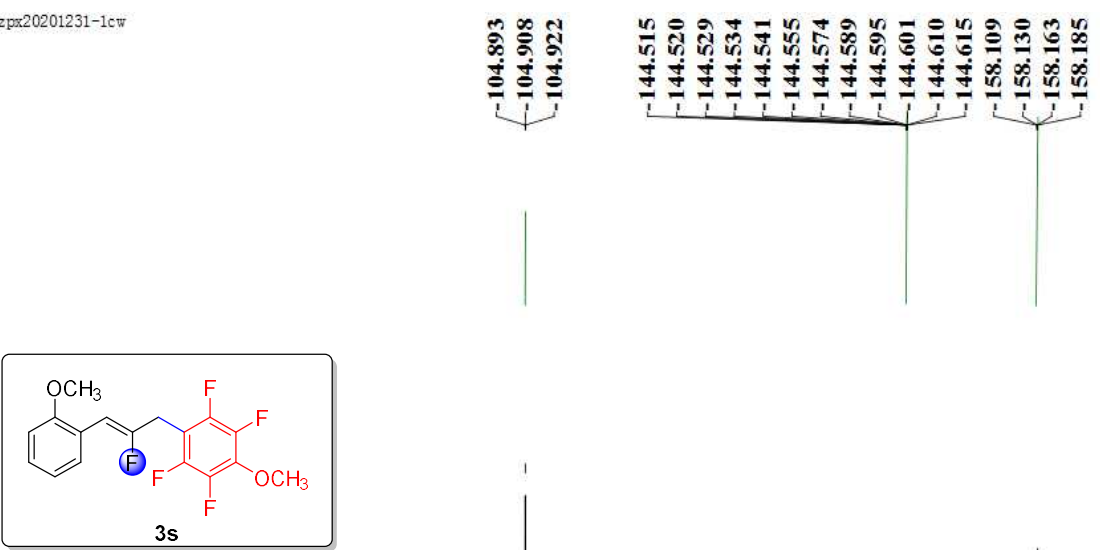

${ }^{19} \mathrm{~F} \mathrm{NMR}\left(376 \mathrm{MHz}, \mathrm{CDC}_{3}\right)$

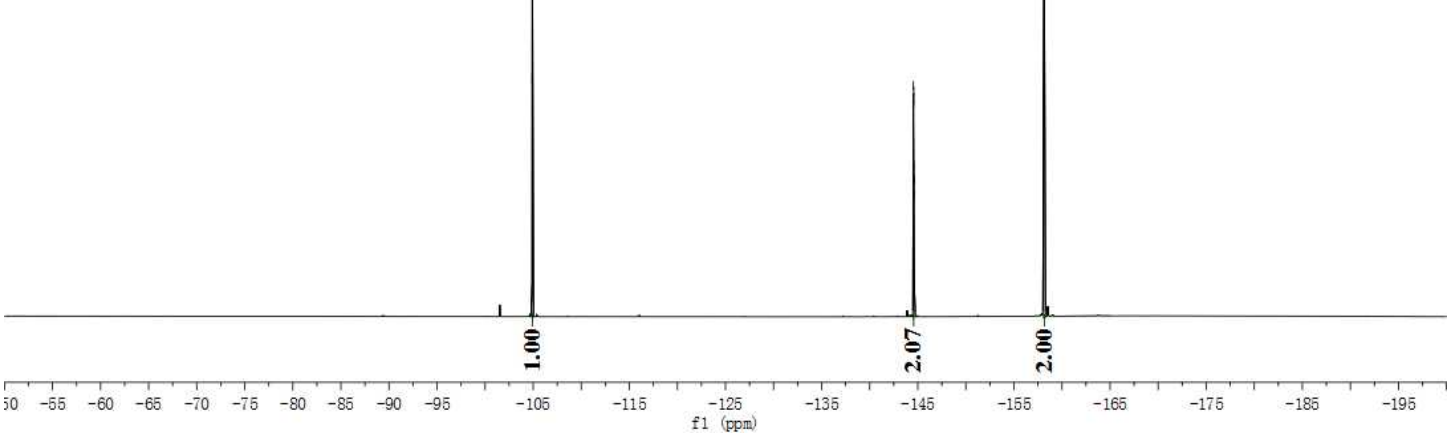




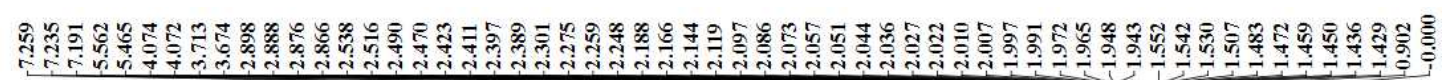

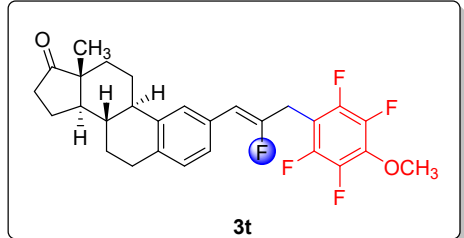

${ }^{1} \mathrm{H} \mathrm{NMR}\left(400 \mathrm{MHz}, \mathrm{CDCl}_{3}\right)$

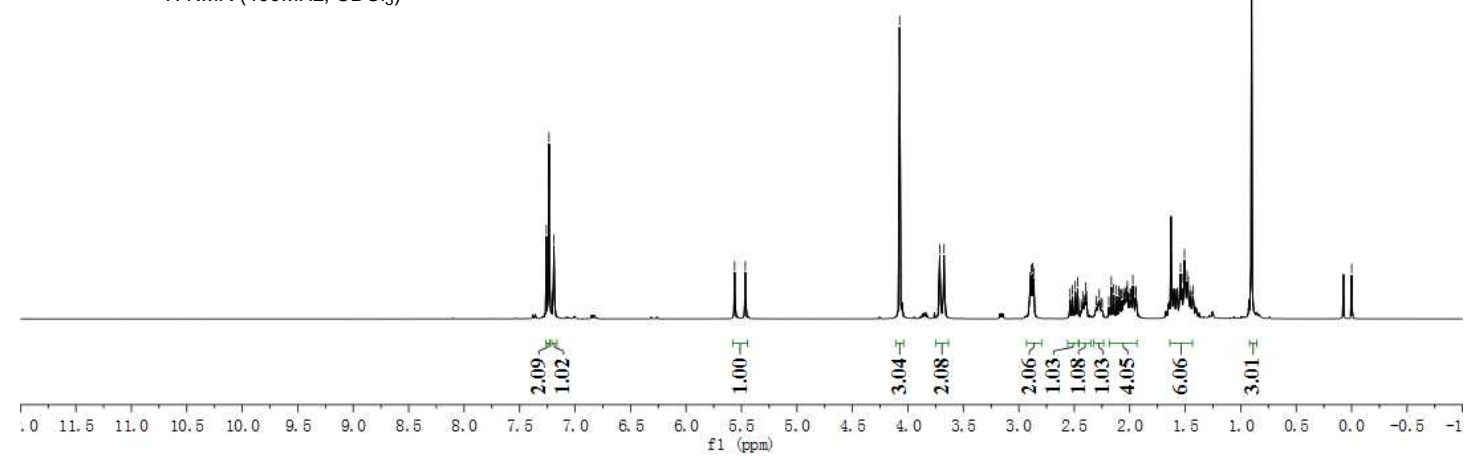

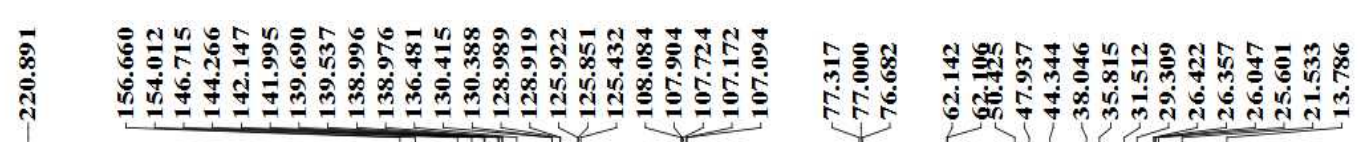

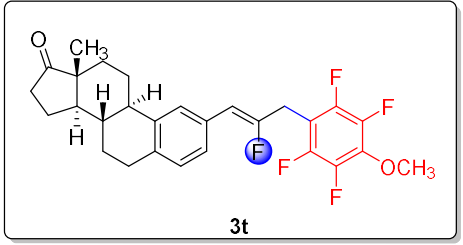

${ }^{13} \mathrm{C} \mathrm{NMR}\left(100 \mathrm{MHz}, \mathrm{CDC}_{3}\right)$

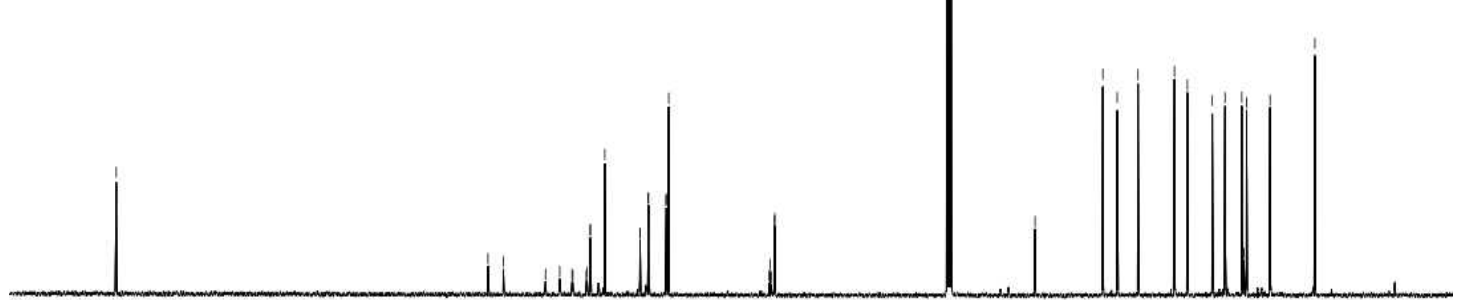

$\begin{array}{llllllllllllllllllllllllllllllllll}230 & 220 & 210 & 200 & 190 & 180 & 170 & 160 & 150 & 140 & 130 & 120 & 110 & 100 & 90 & 80 & 70 & 60 & 50 & 40 & 30 & 20 & 10 & 0 & -1\end{array}$ 

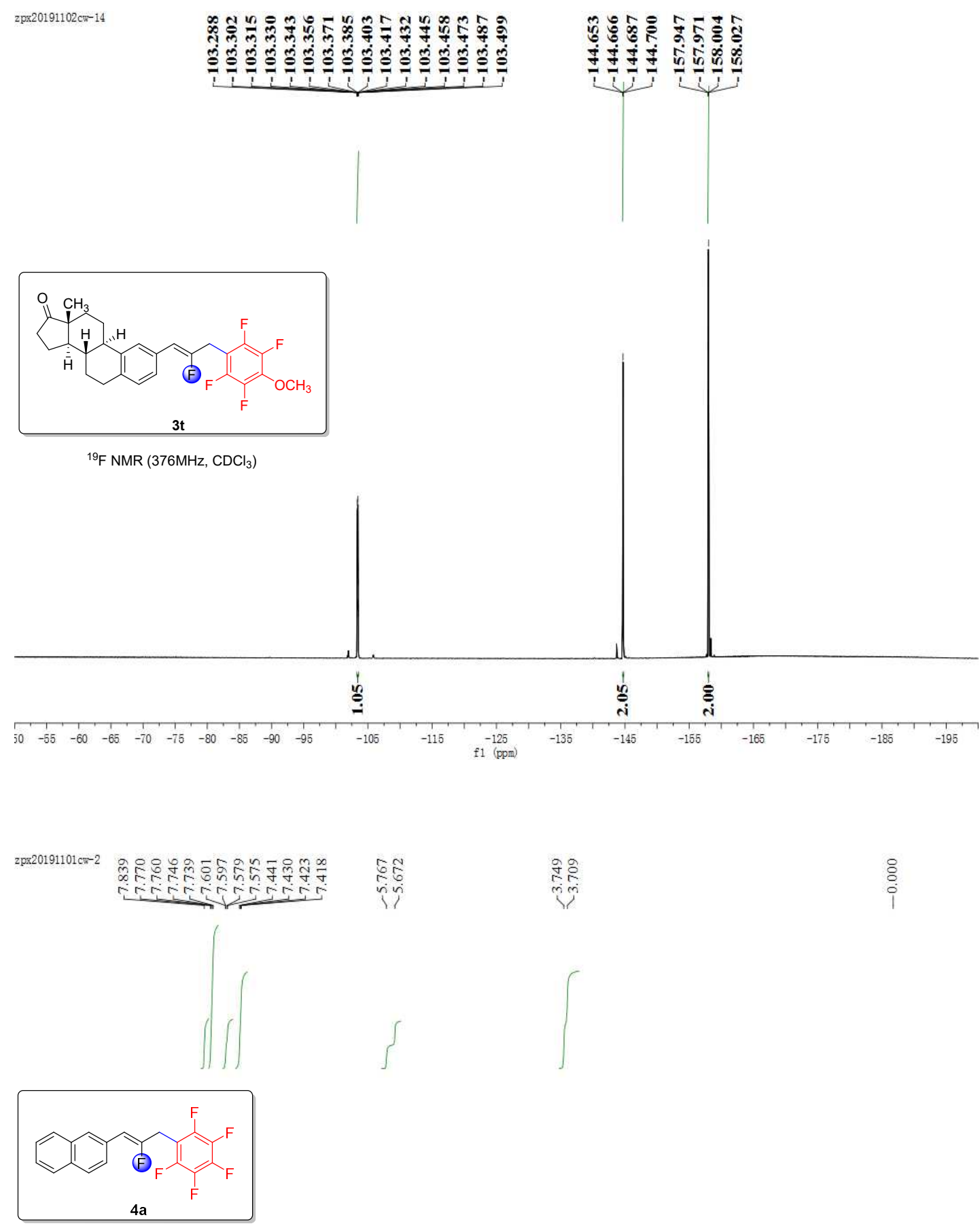

${ }^{1} \mathrm{H} \mathrm{NMR}\left(400 \mathrm{MHz}, \mathrm{CDCl}_{3}\right)$

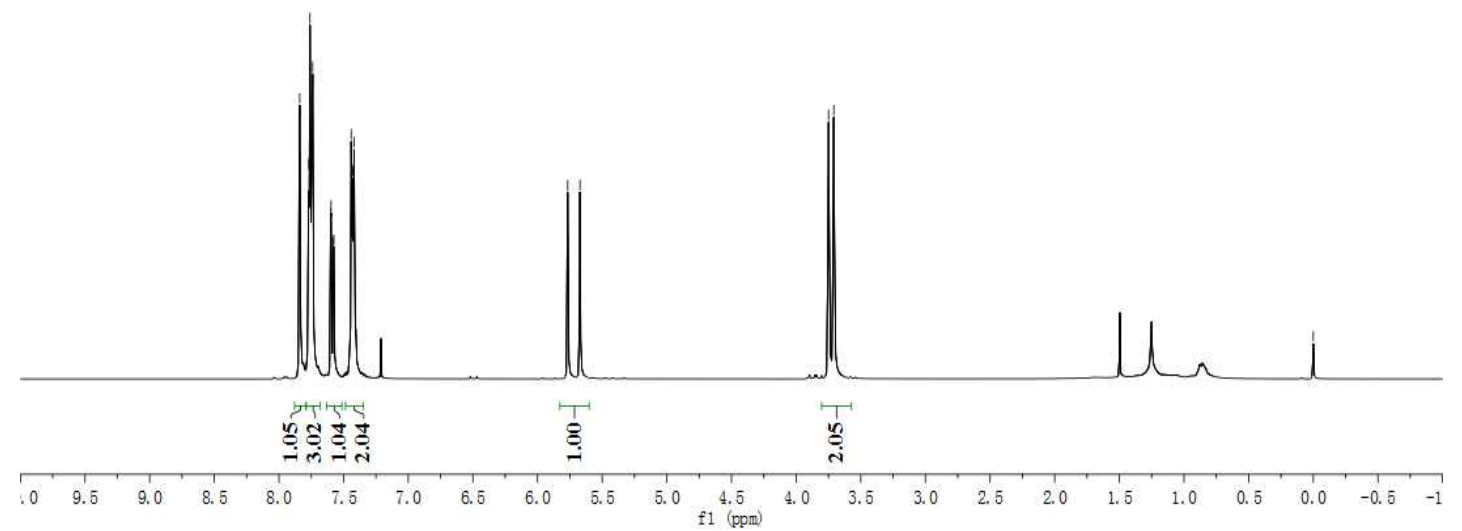




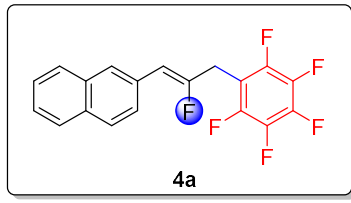

$\left.{ }^{13} \mathrm{C} \mathrm{NMR} \mathrm{(100} \mathrm{MHz,} \mathrm{CDCl}_{3}\right)$
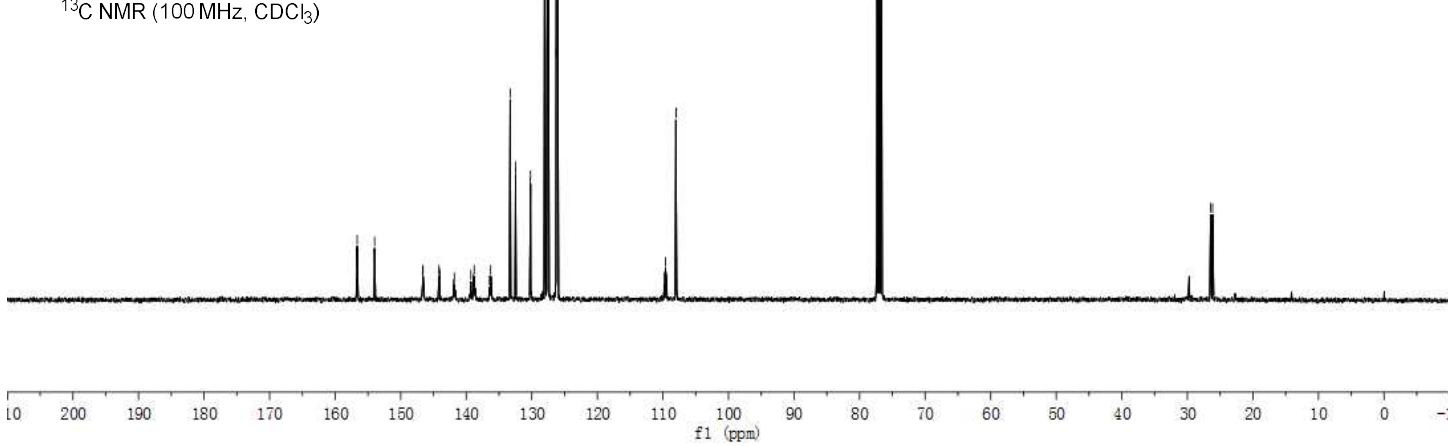

zpx20191101 cw-2
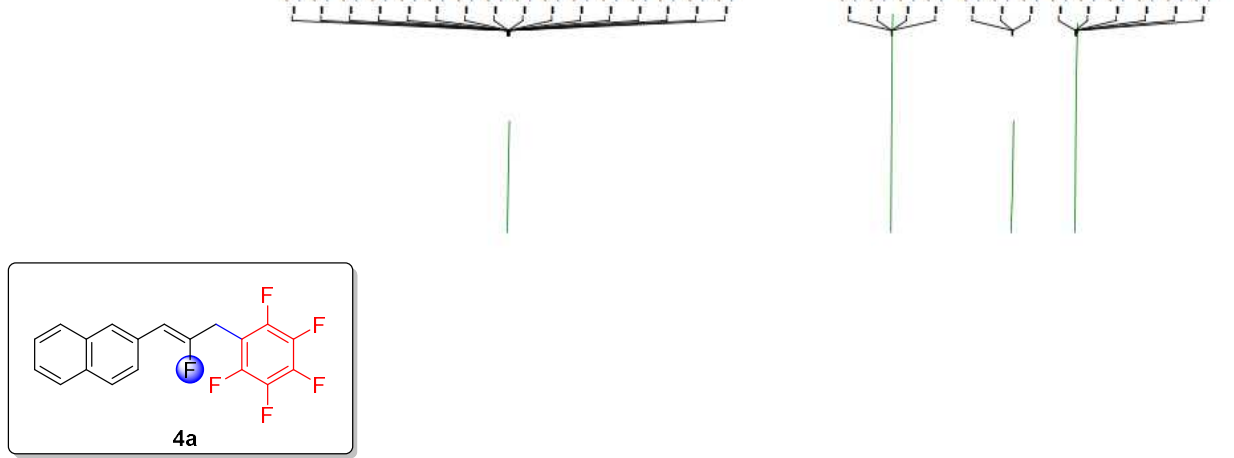

$\left.{ }^{19} \mathrm{~F} \mathrm{NMR} \mathrm{(376MHz,} \mathrm{CDCl} 3\right)$

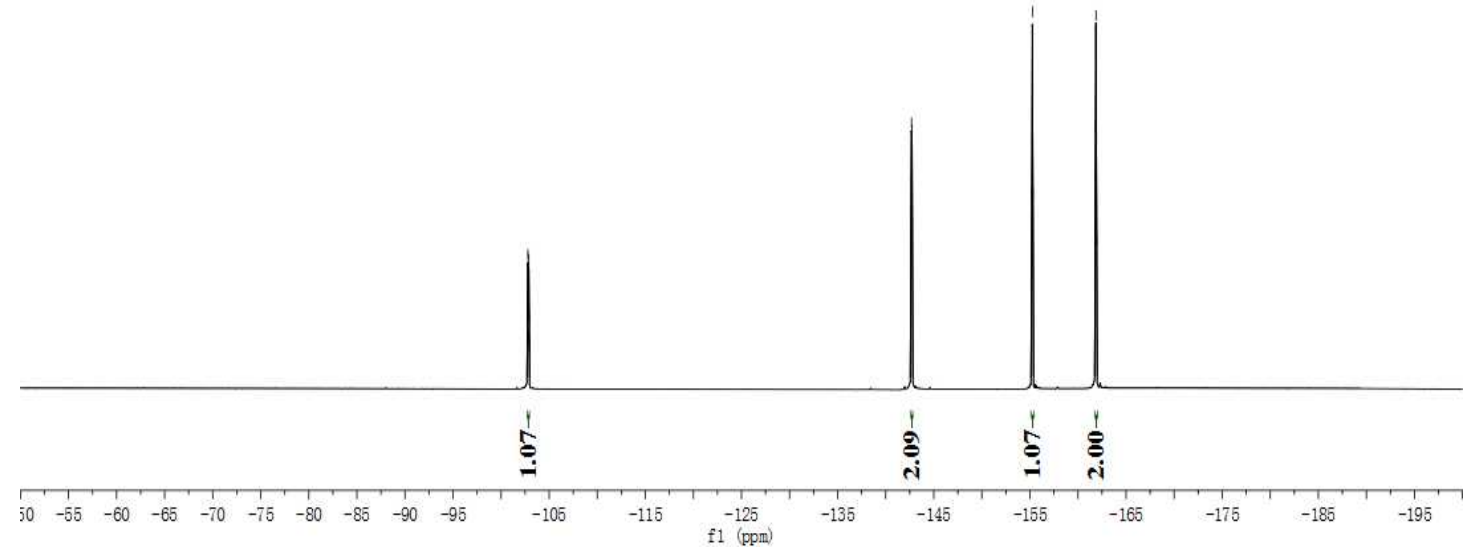



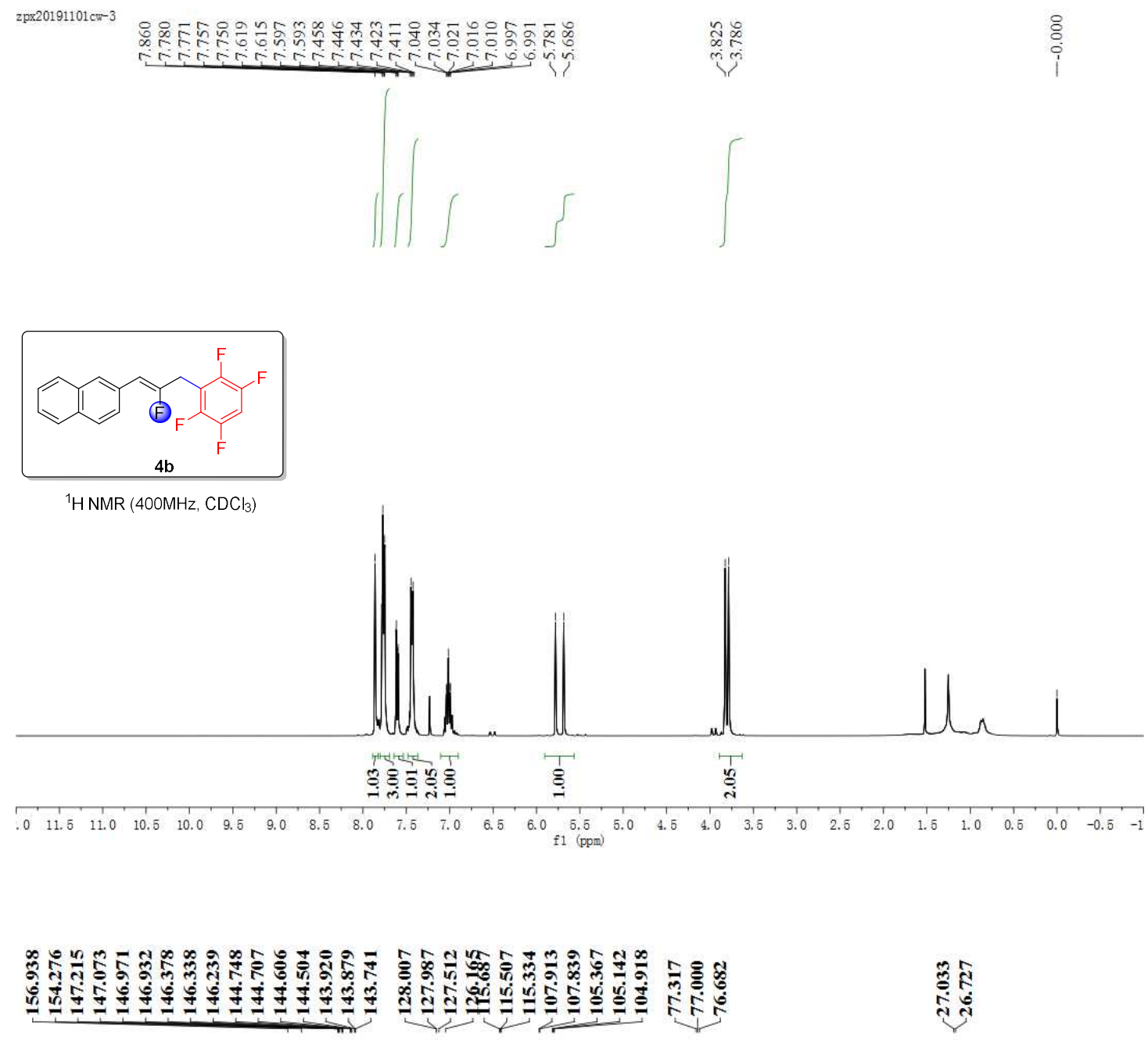

है

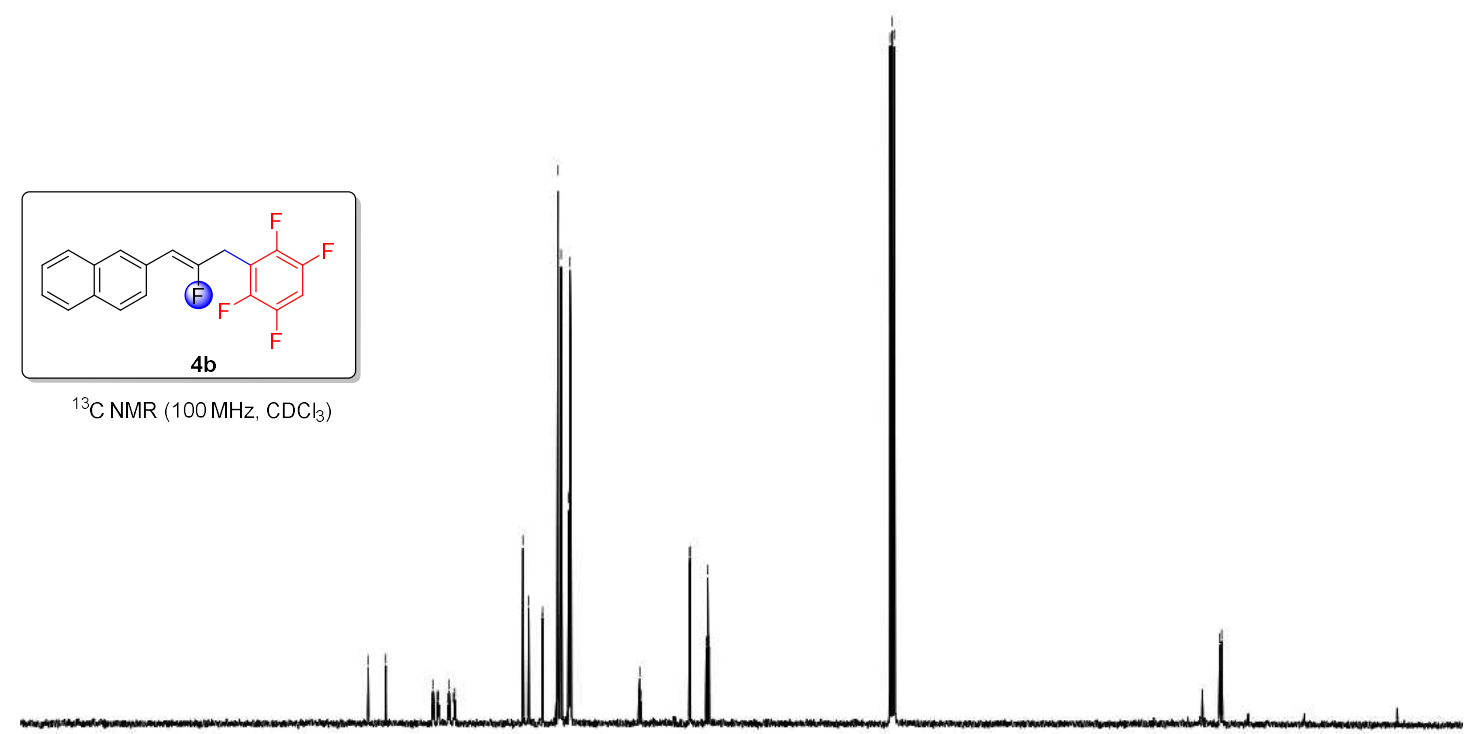

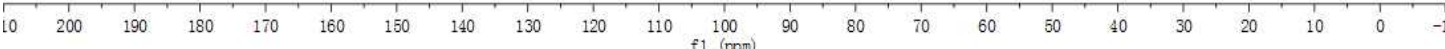




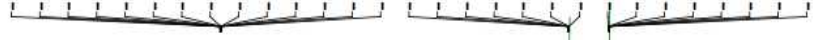

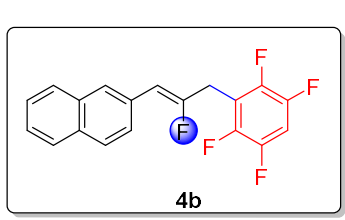

${ }^{19} \mathrm{~F} \mathrm{NMR} \mathrm{(376MHz,} \mathrm{CDCla)}$ 造
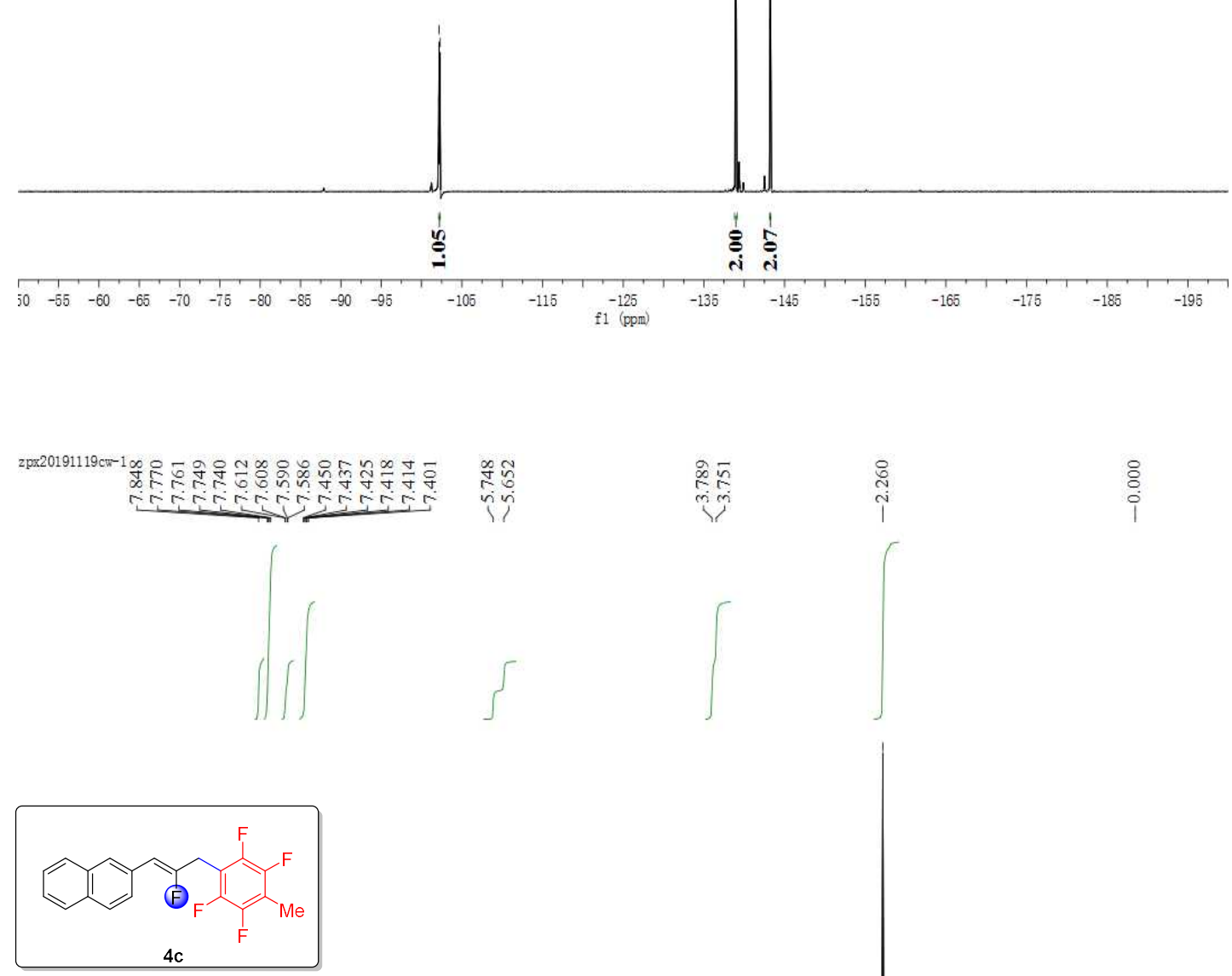

${ }^{1} \mathrm{HNMR}\left(400 \mathrm{MHz}, \mathrm{CDC}_{3}\right)$

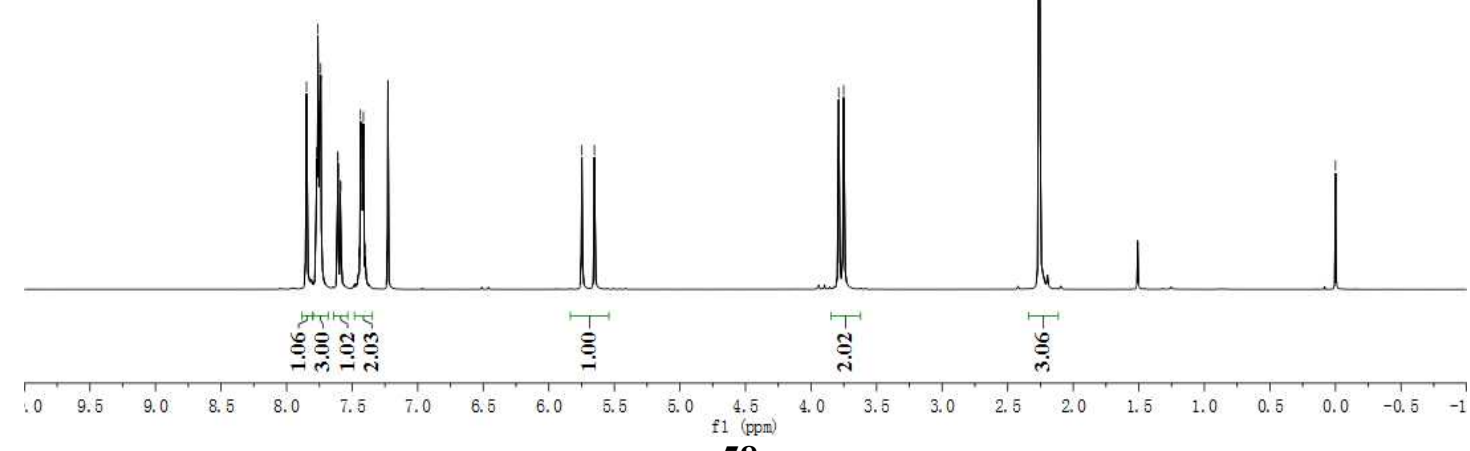




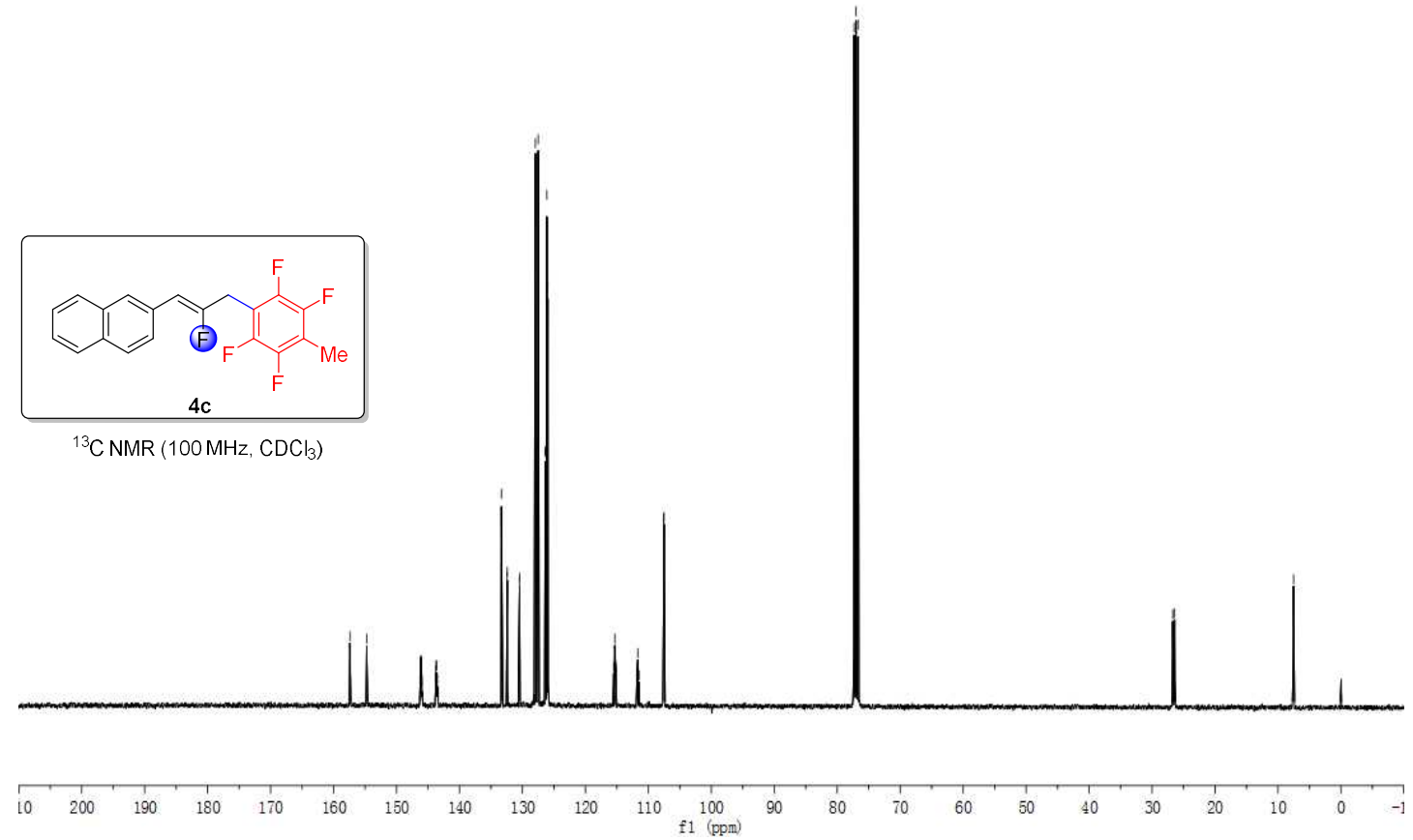

$20191119 \mathrm{cw}-1$

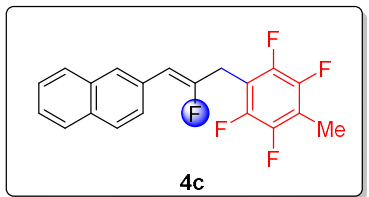

${ }^{19} \mathrm{~F} \mathrm{NMR}\left(376 \mathrm{MHz}, \mathrm{CDC}_{3}\right)$
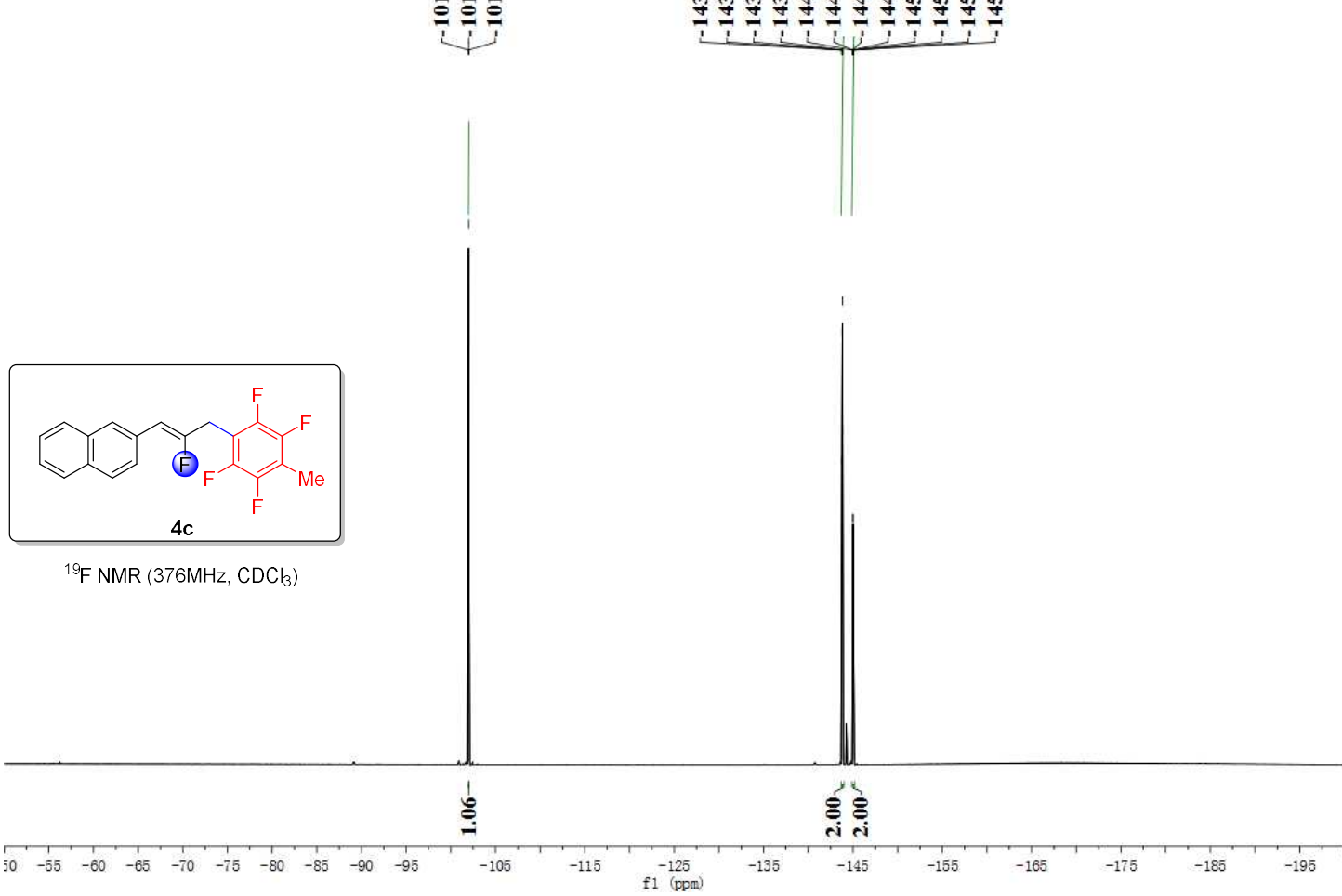


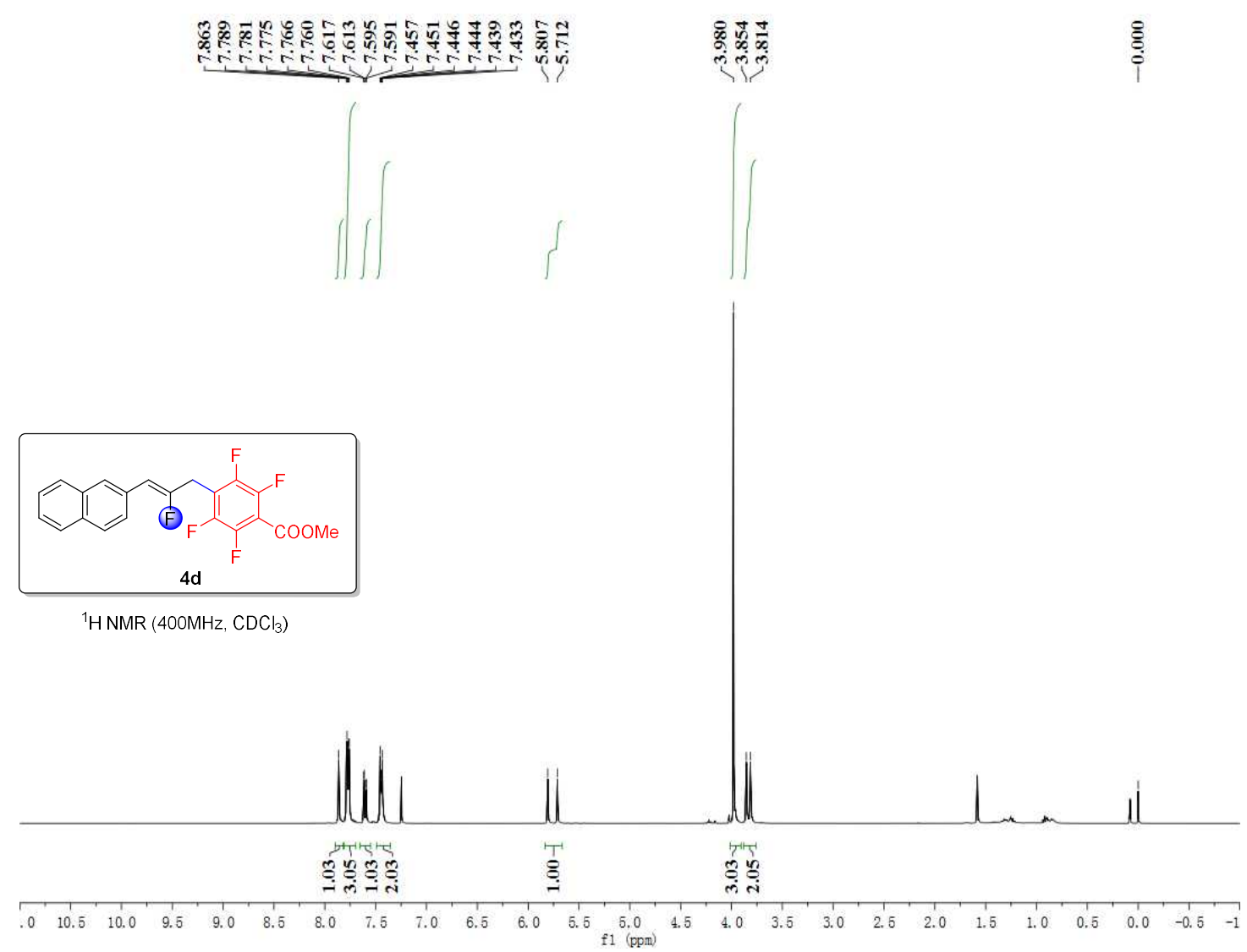

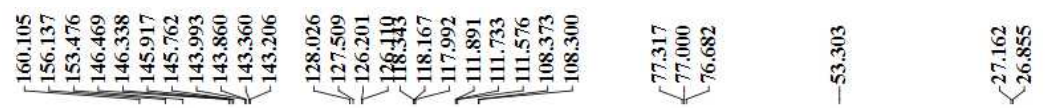

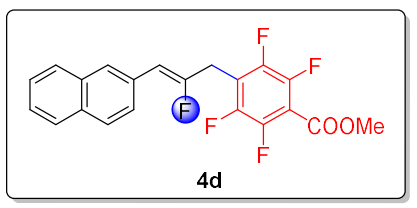

${ }^{13} \mathrm{C} \mathrm{NMR}\left(100 \mathrm{MHz}, \mathrm{CDCl}_{3}\right)$

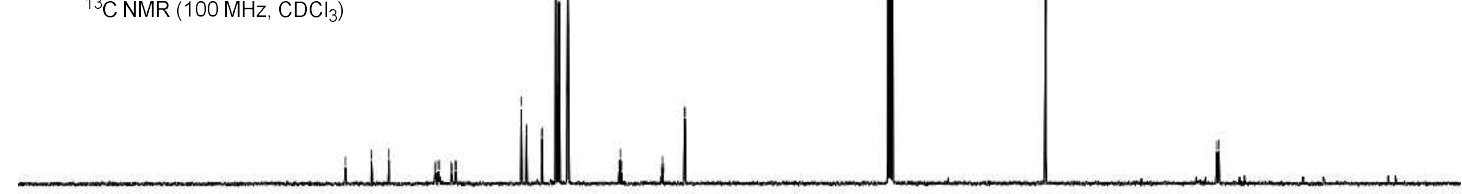

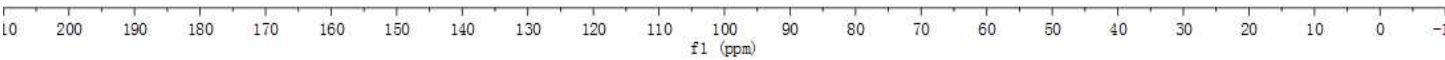




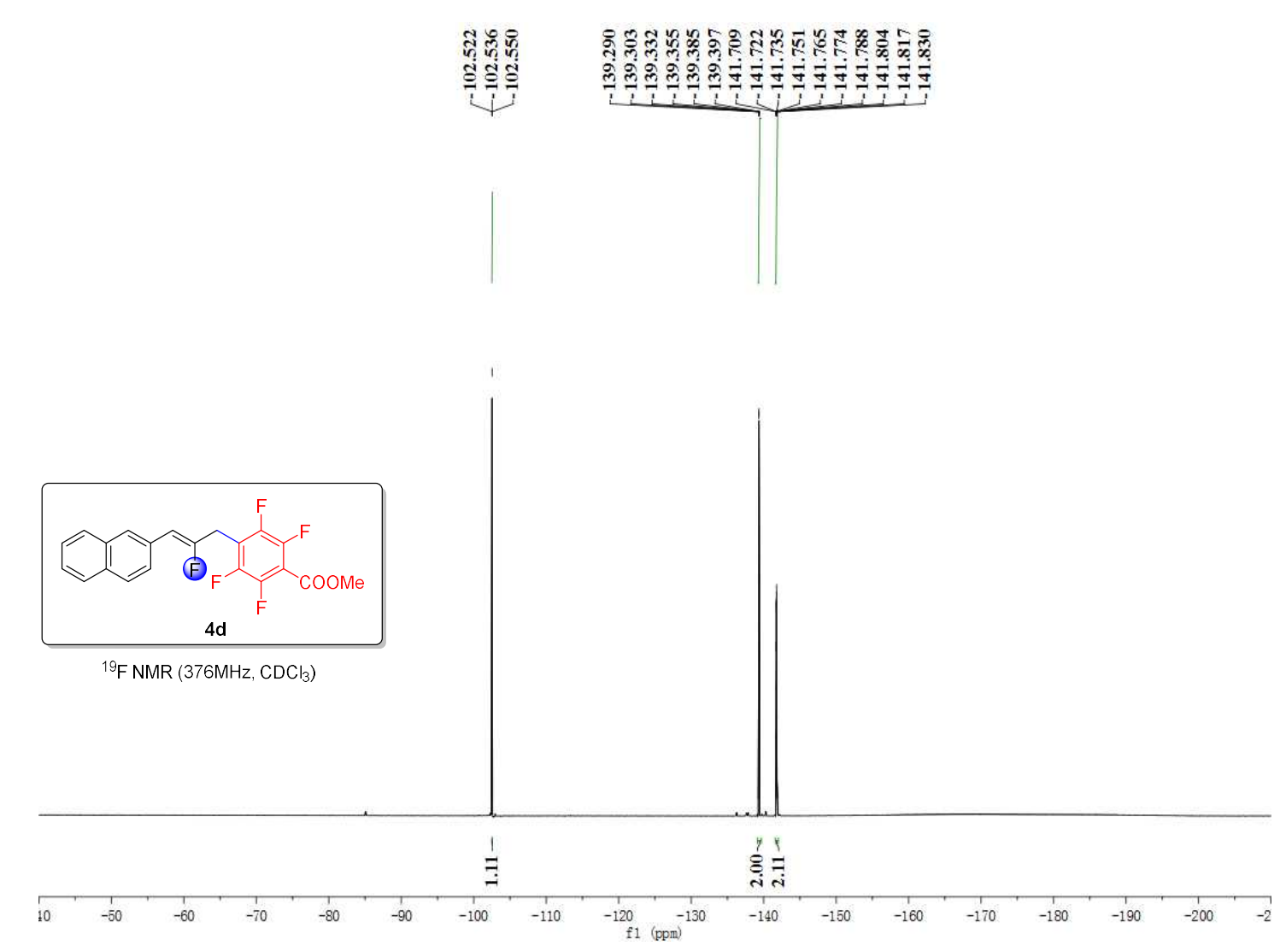

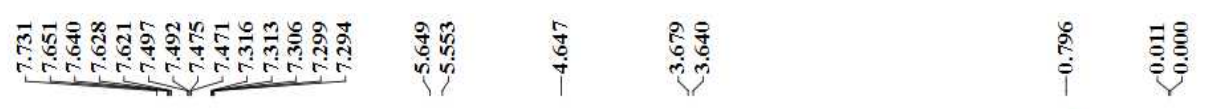

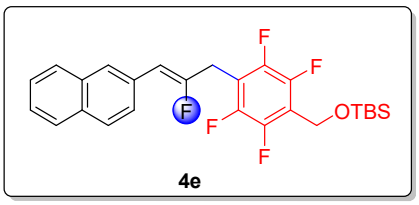

${ }^{1} \mathrm{H}$ NMR (400MHz, $\mathrm{CDCl}_{3}$ )

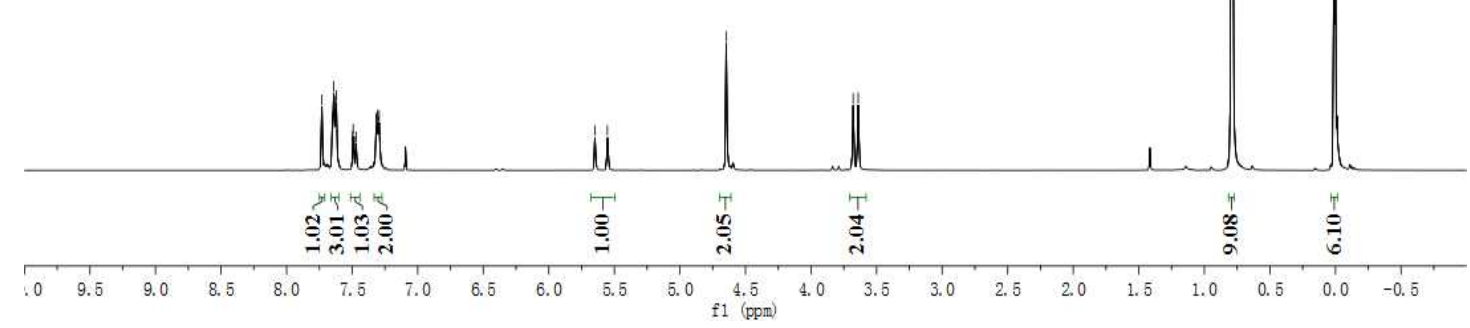




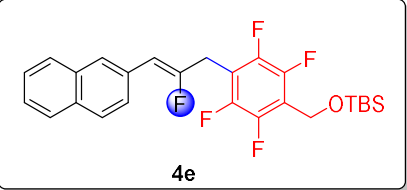

$\left.{ }^{13} \mathrm{C} \mathrm{NMR} \mathrm{(100} \mathrm{MHz,} \mathrm{CDCl}_{3}\right)$

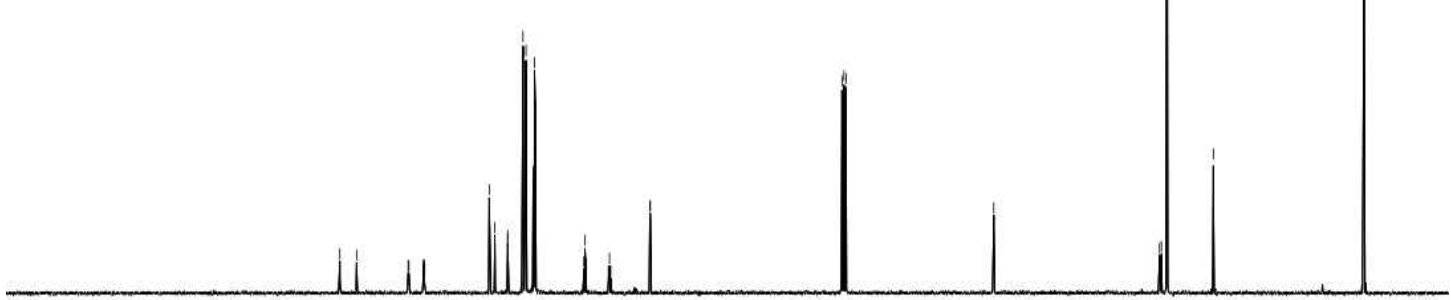

\begin{tabular}{llllllllllllllllllllllll}
\hline 0 & 200 & 190 & 180 & 170 & 160 & 150 & 140 & 130 & 120 & 110 & 100 & 90 & 80 & 70 & 60 & 50 & 40 & 30 & 20 & 10 & 0 & -10
\end{tabular}

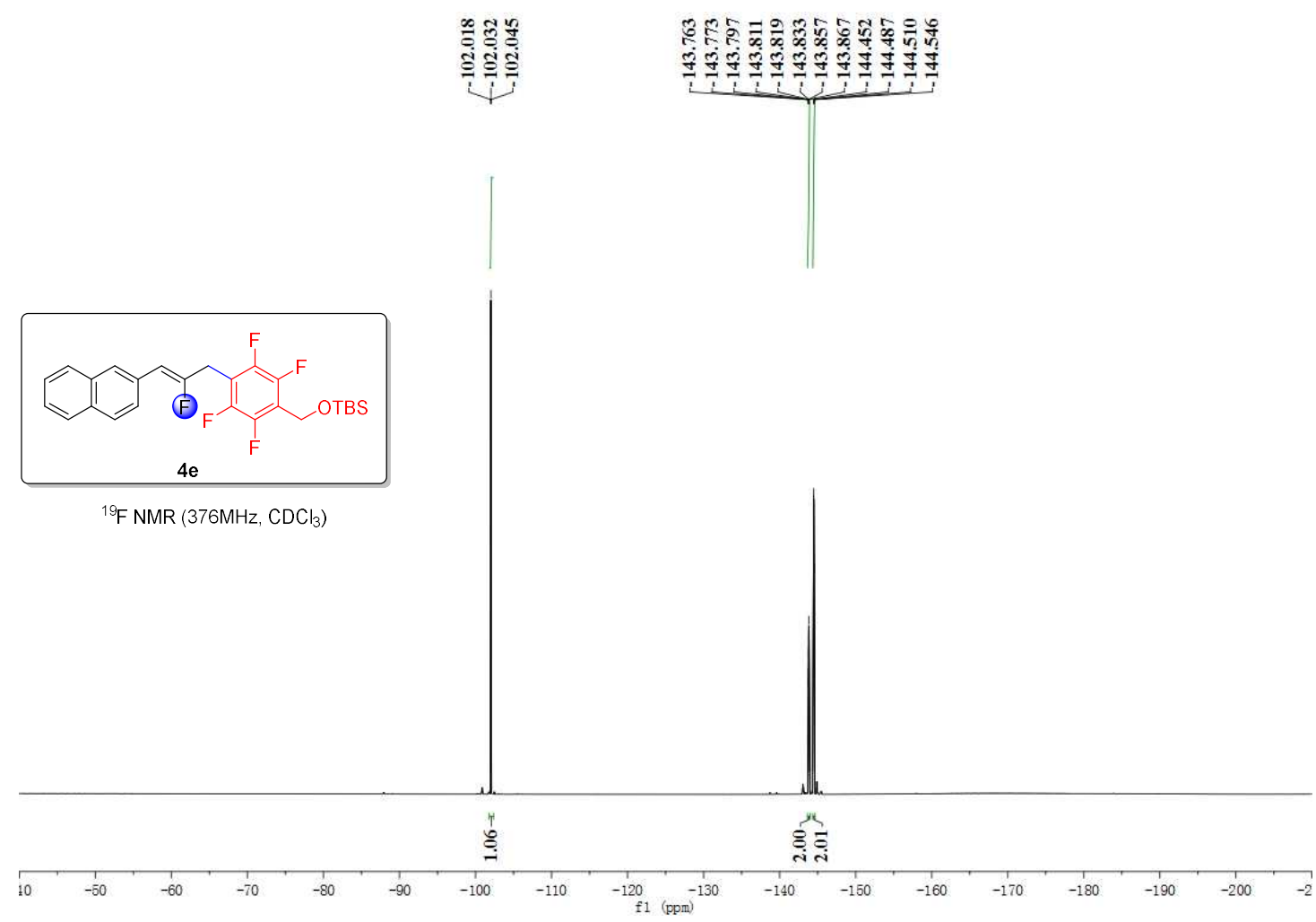



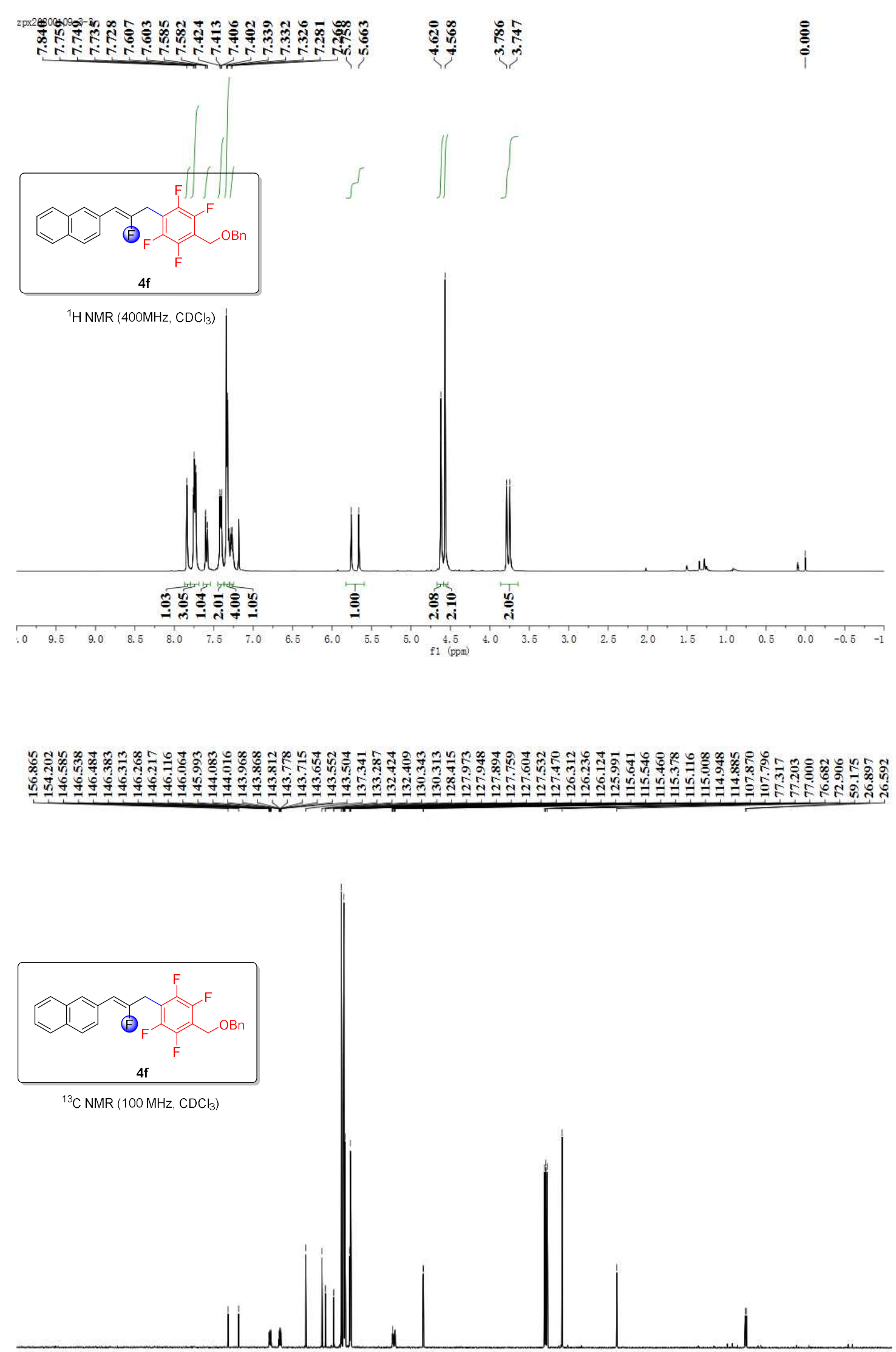

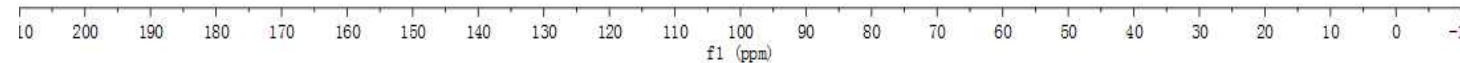



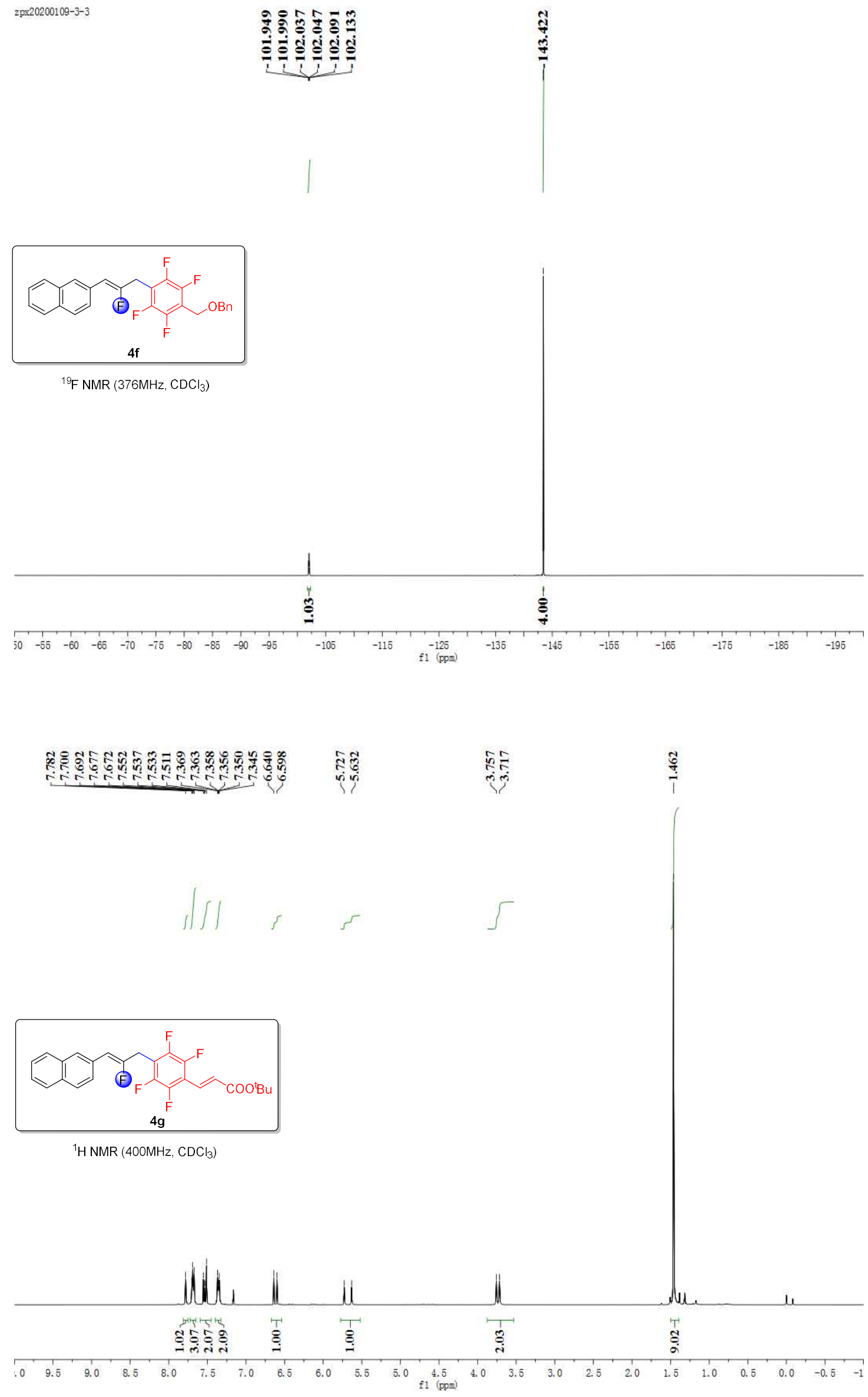

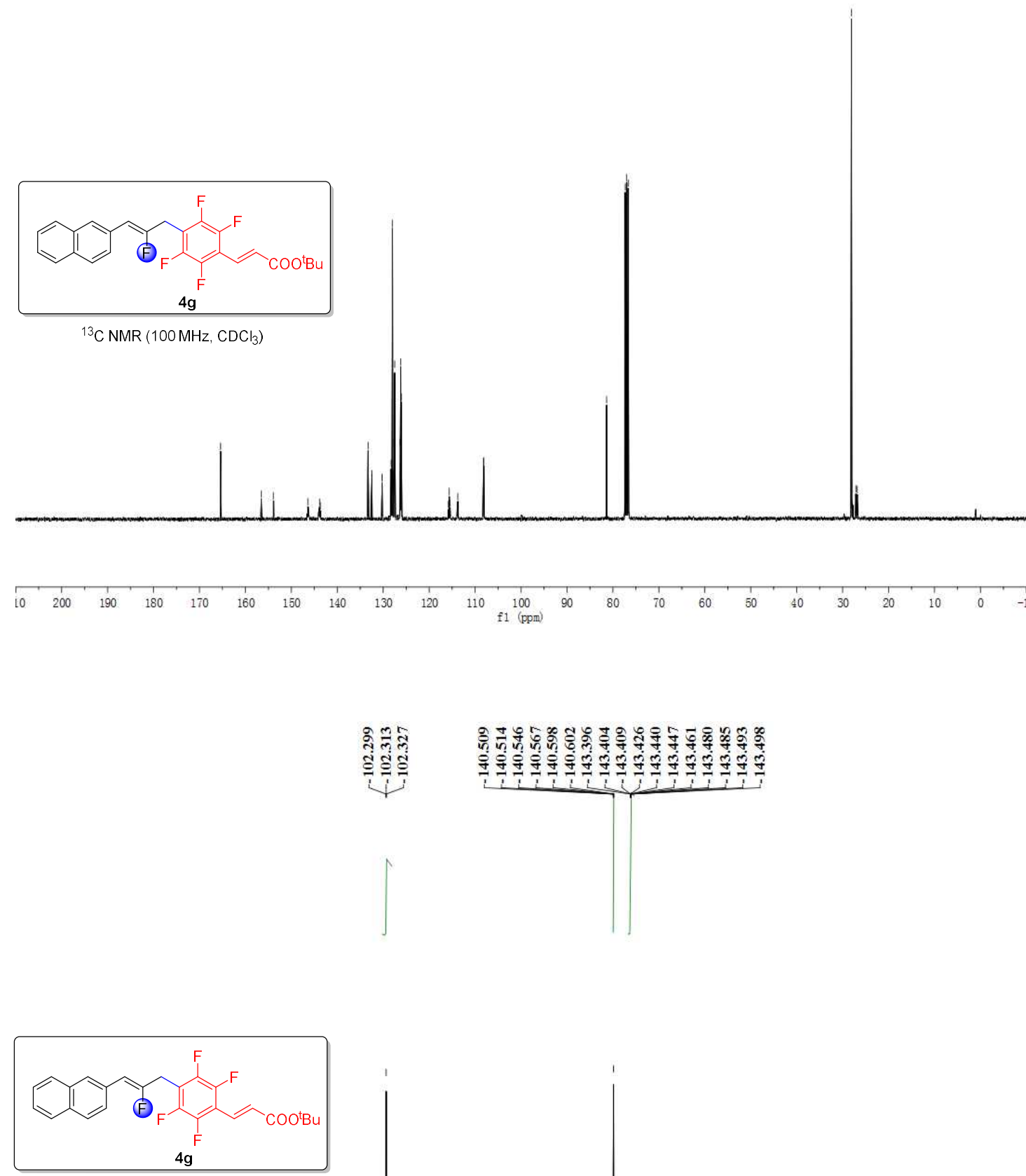

${ }^{19} \mathrm{~F} \mathrm{NMR}\left(376 \mathrm{MHz} \quad \mathrm{CDCl}_{3}\right)$

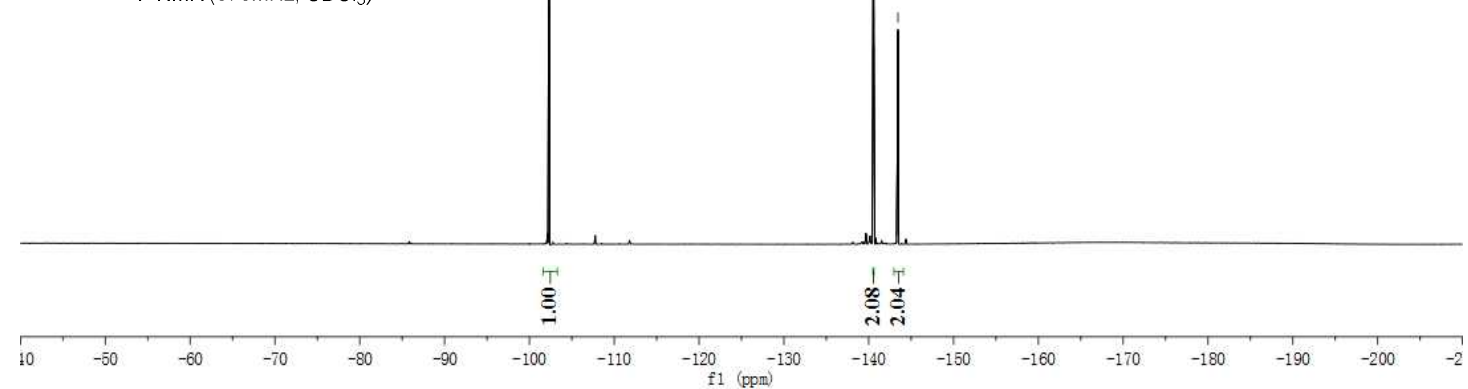



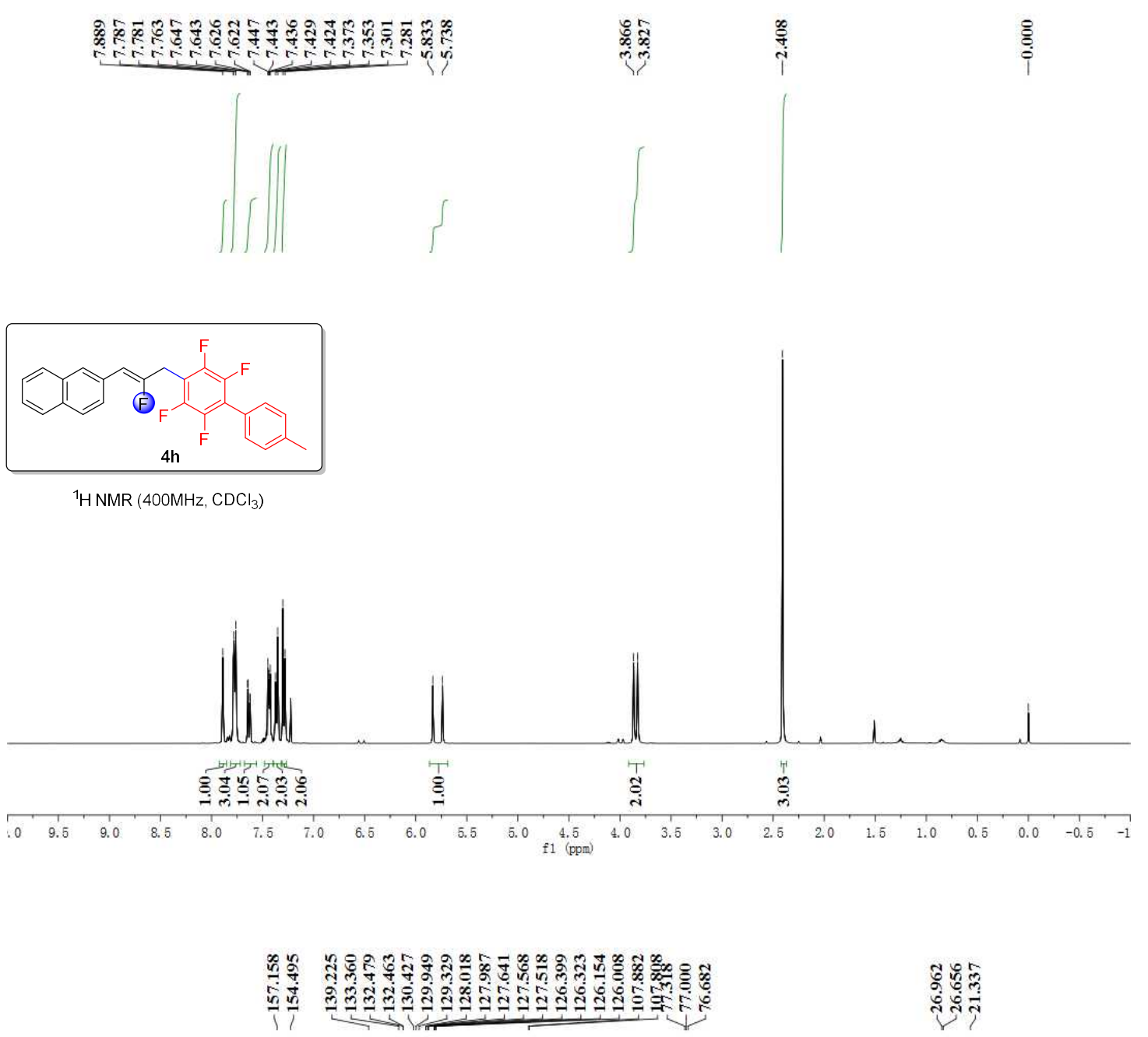

影

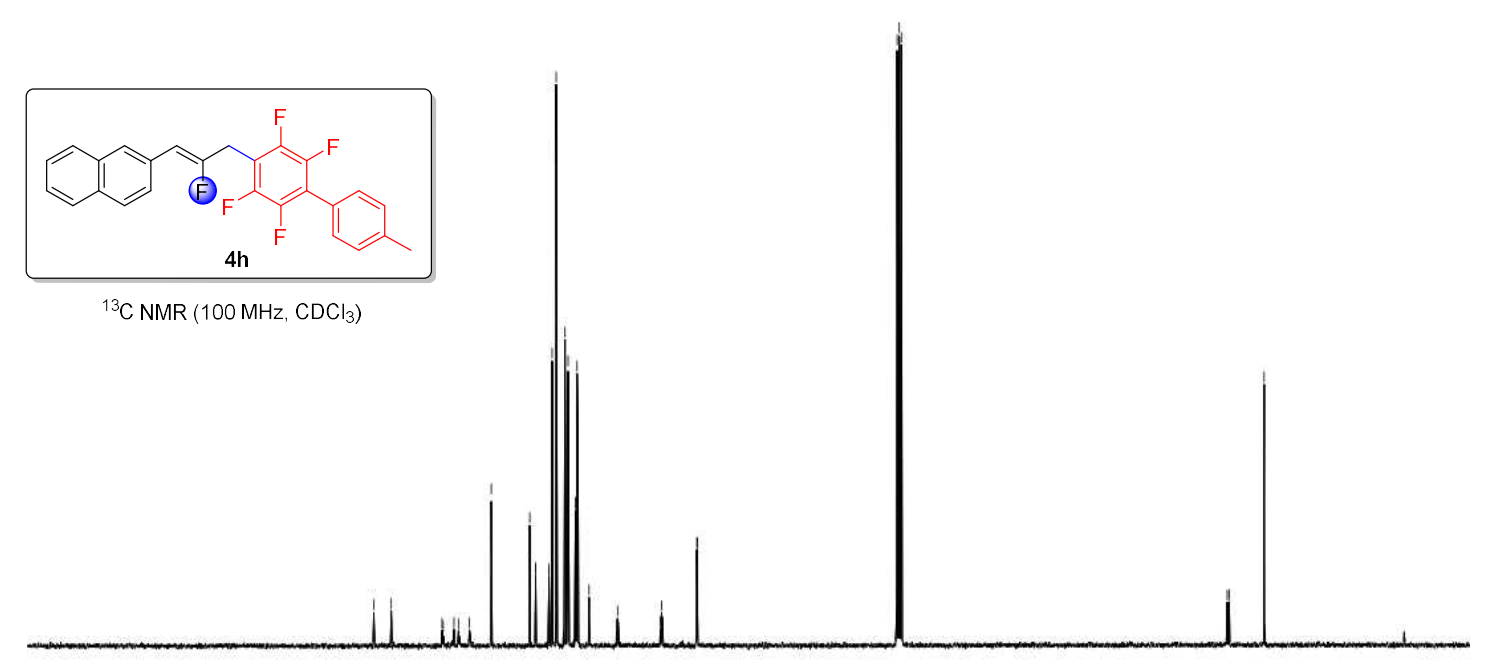

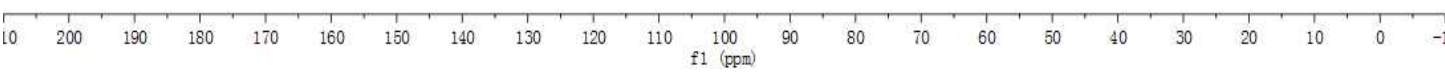



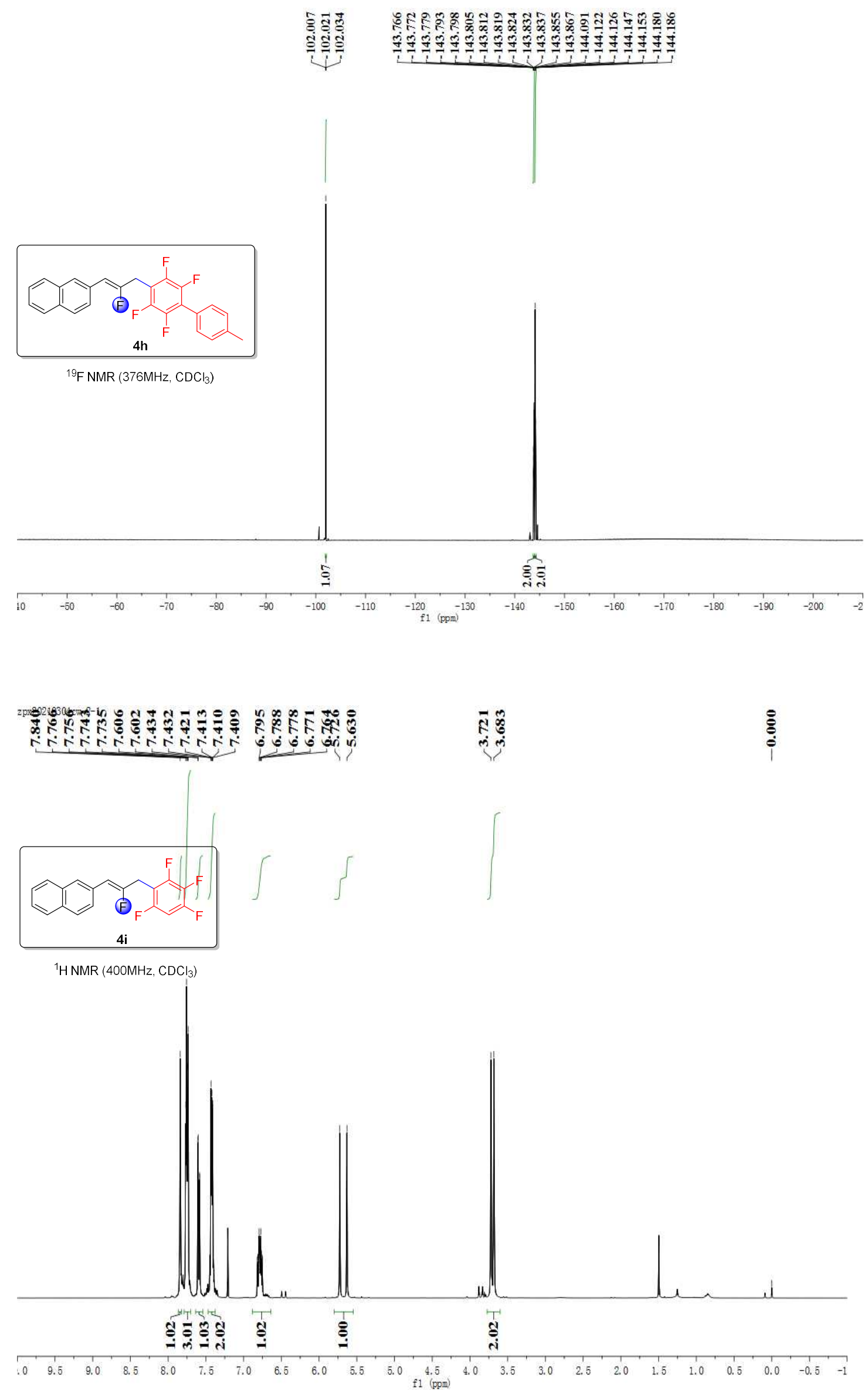


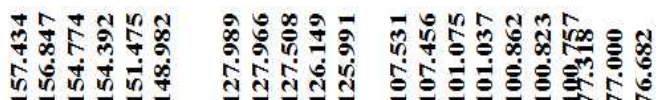

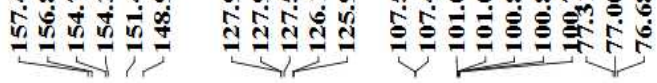

ฝู่

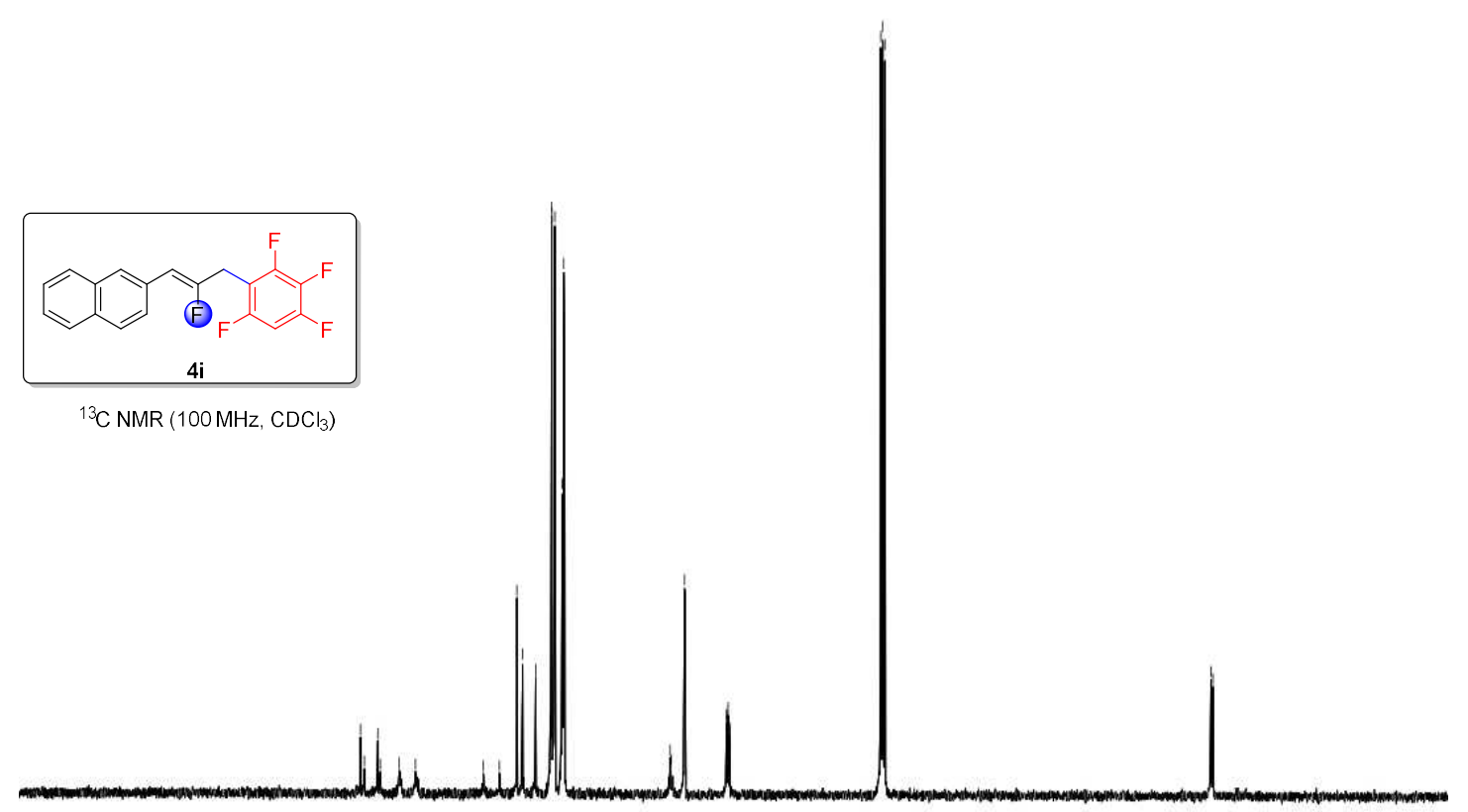

zpr20210304cw-2-1
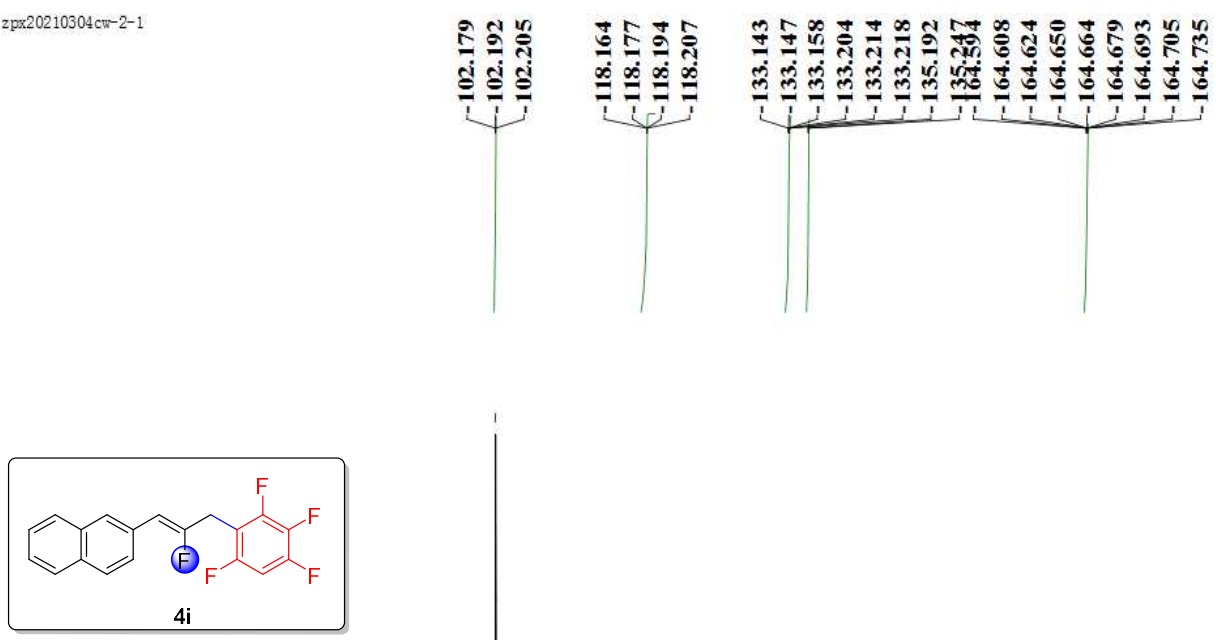

${ }^{19} \mathrm{~F} \mathrm{NMR}\left(376 \mathrm{MHz}, \mathrm{CDC}_{3}\right)$

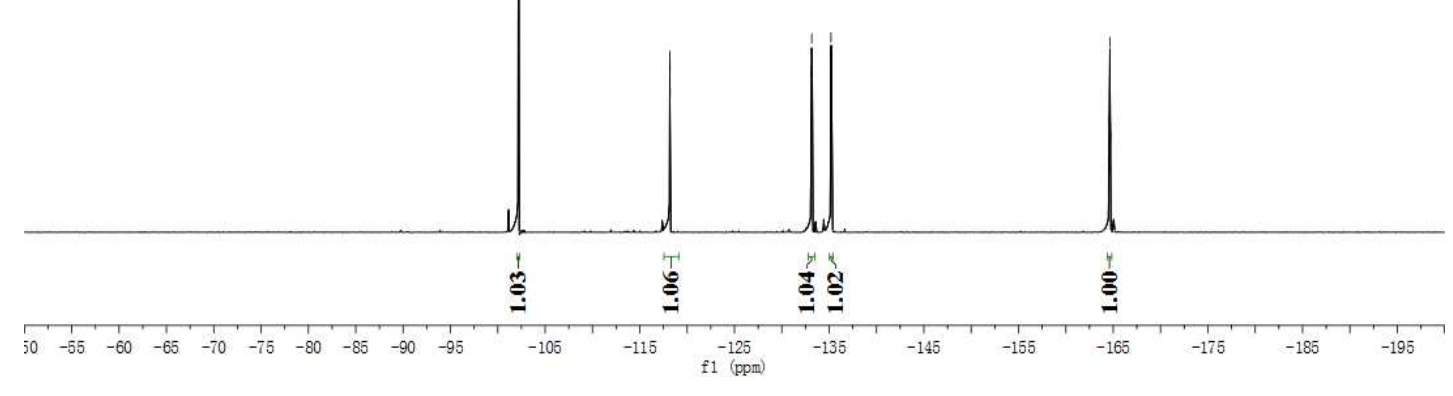



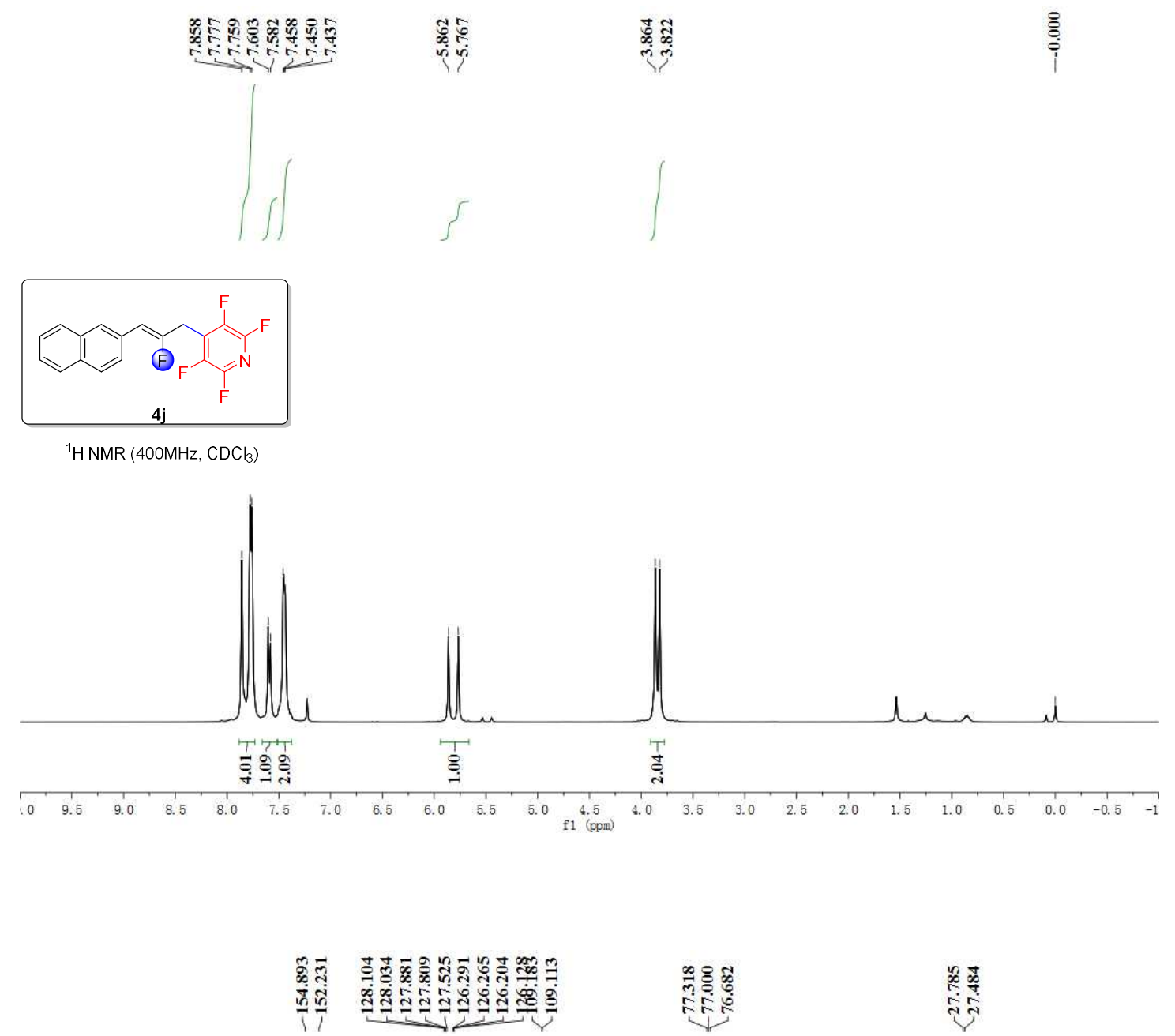

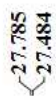

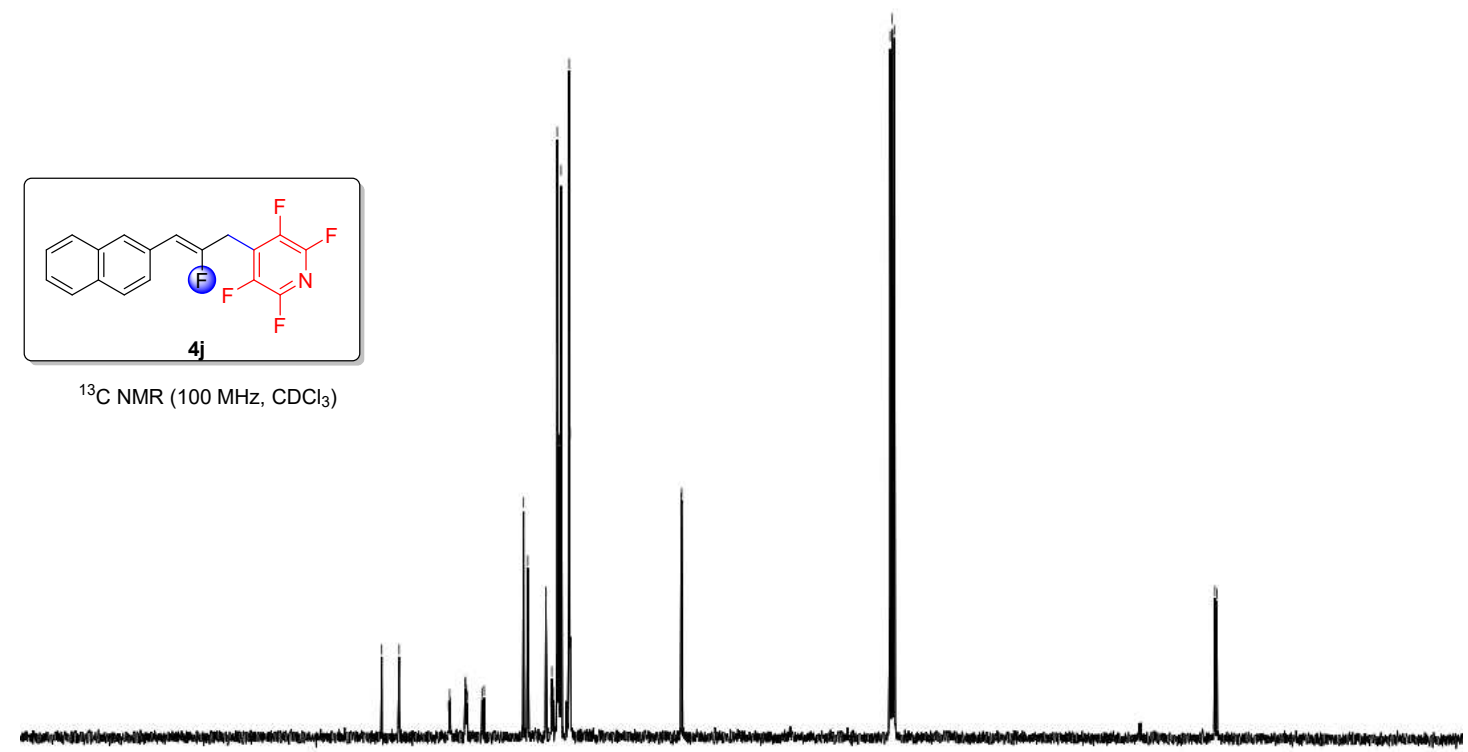

$\left.{ }^{13} \mathrm{C} \mathrm{NMR} \mathrm{(100} \mathrm{MHz,} \mathrm{CDCl}_{3}\right)$

$\begin{array}{lllllllllllllllllllllllllllllllllllllllllll}10 & 200 & 190 & 180 & 170 & 160 & 150 & 140 & 130 & 120 & 110 & 100 & 90 & 80 & 70 & 60 & 50 & 40 & 30 & 20 & 10 & 0 & -1\end{array}$ 

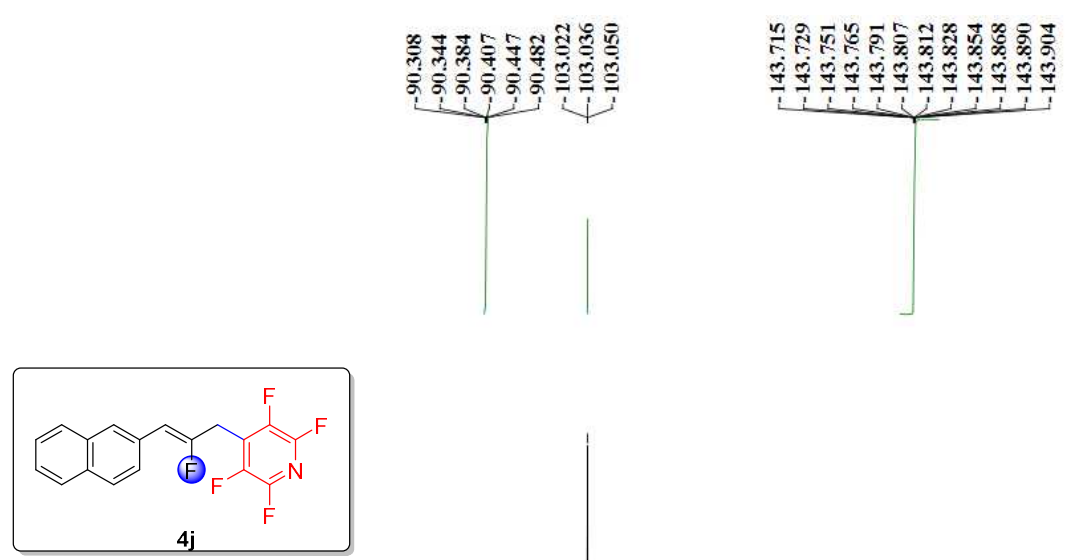

${ }^{1 \mathrm{~F}} \mathrm{~F} \mathrm{NMR}\left(376 \mathrm{MHz}, \mathrm{CDC}_{3}\right)$
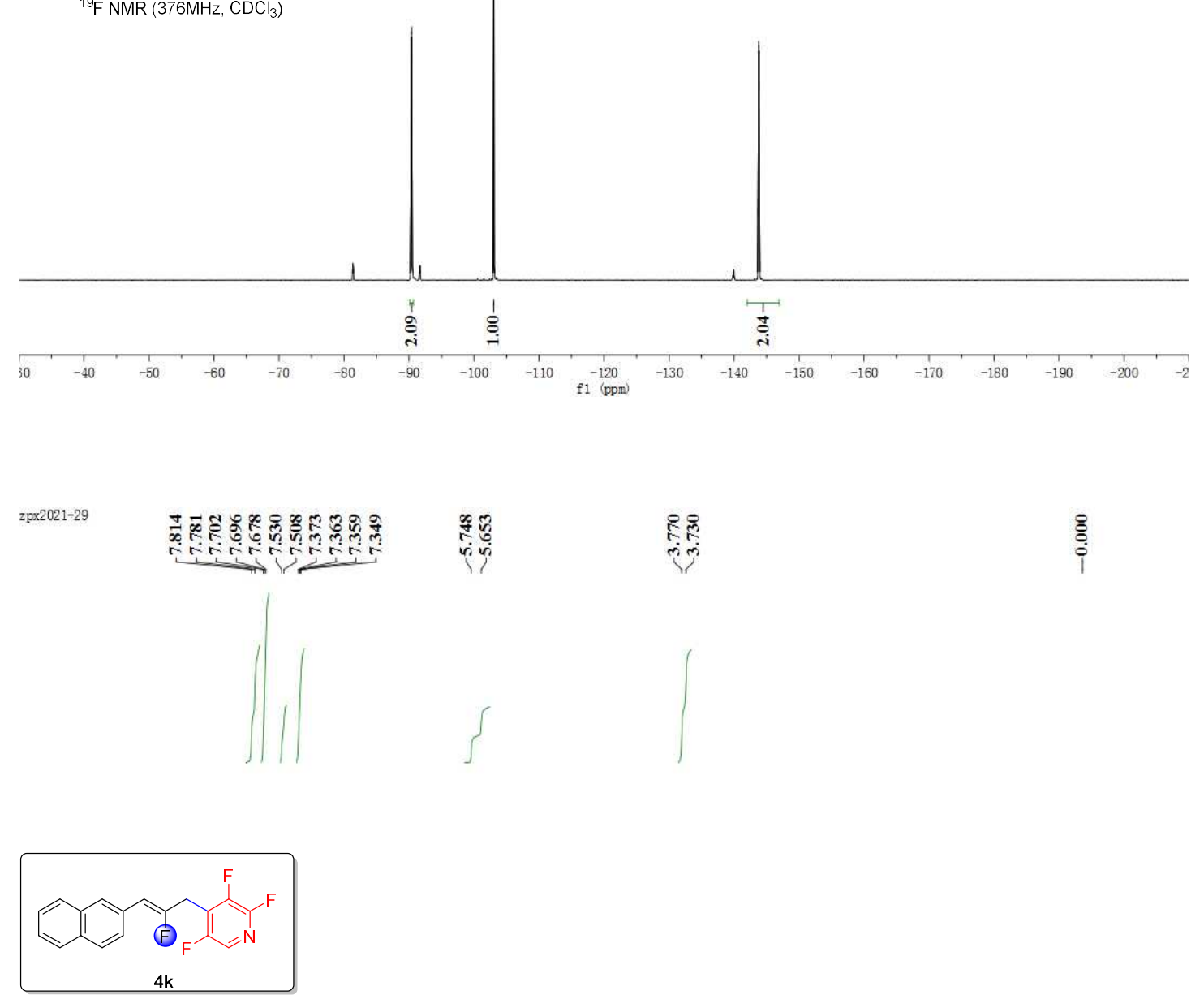

${ }^{1} \mathrm{H} \mathrm{NMR}\left(400 \mathrm{MHz} \quad \mathrm{CDCl}_{3}\right)$

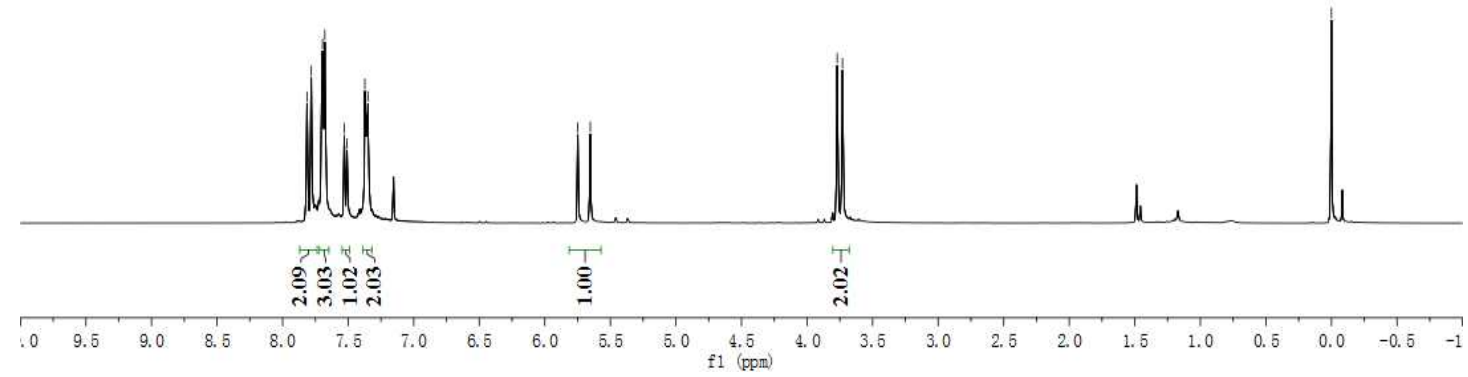



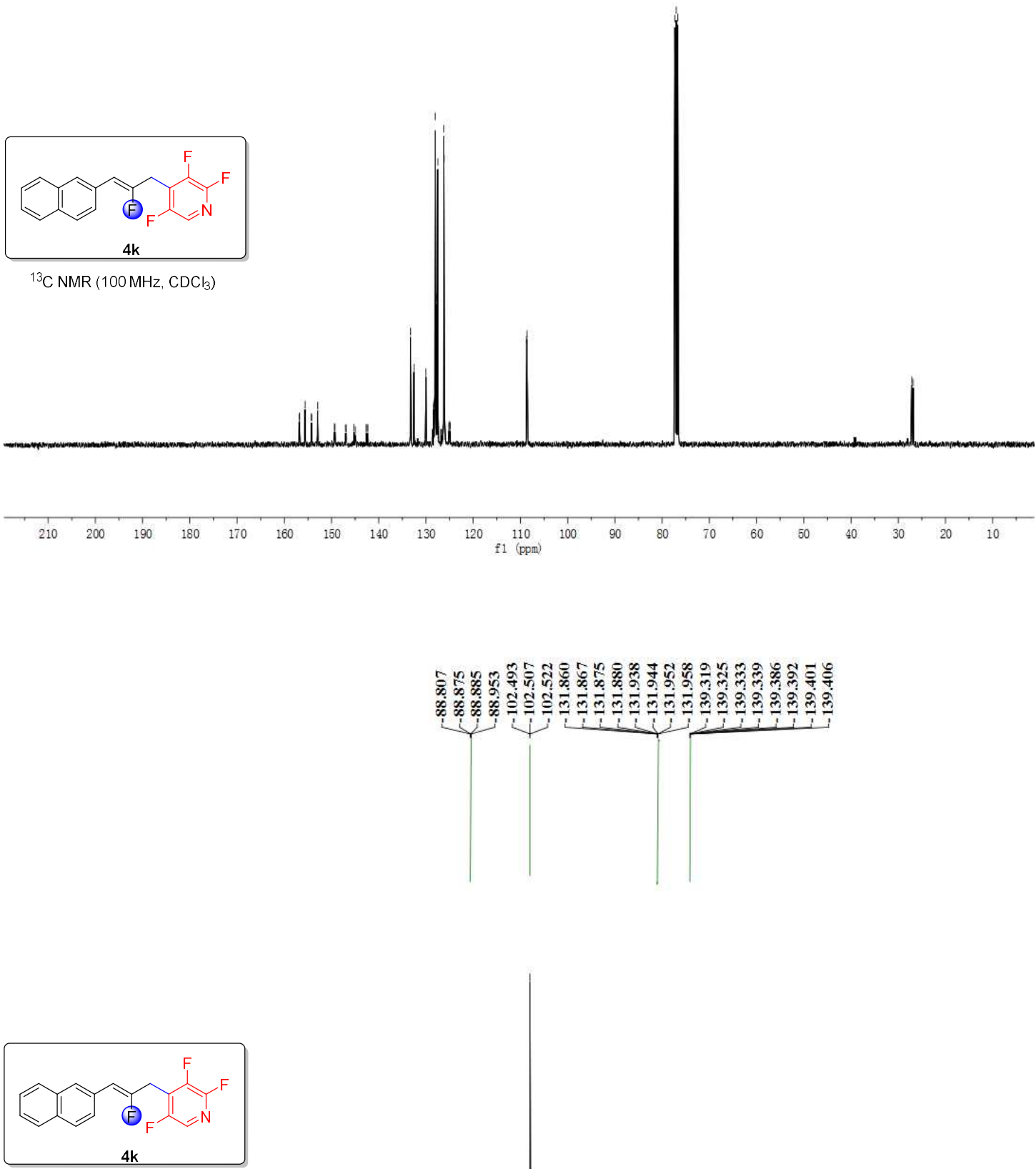

${ }^{15} \mathrm{~F} \mathrm{NMR}(376 \mathrm{MHz}, \mathrm{CDCl} 3)$

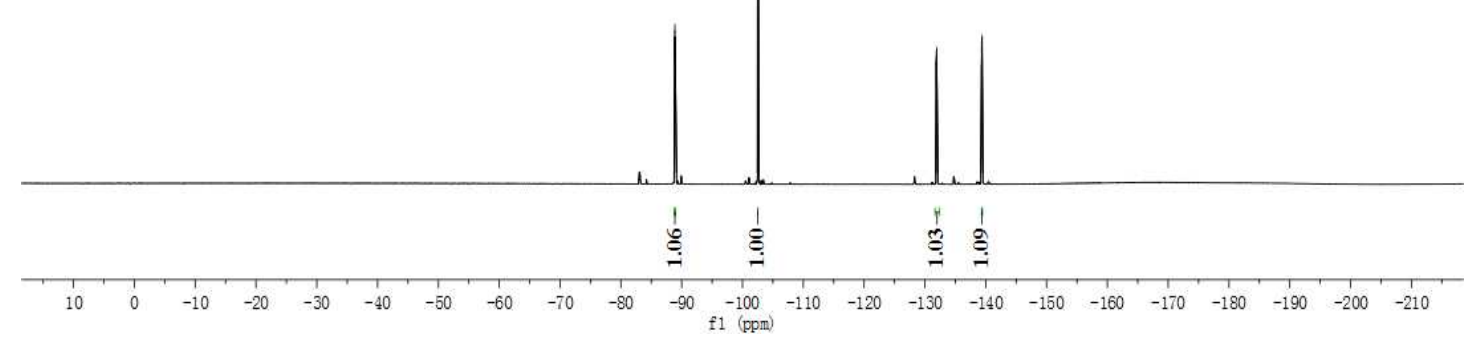



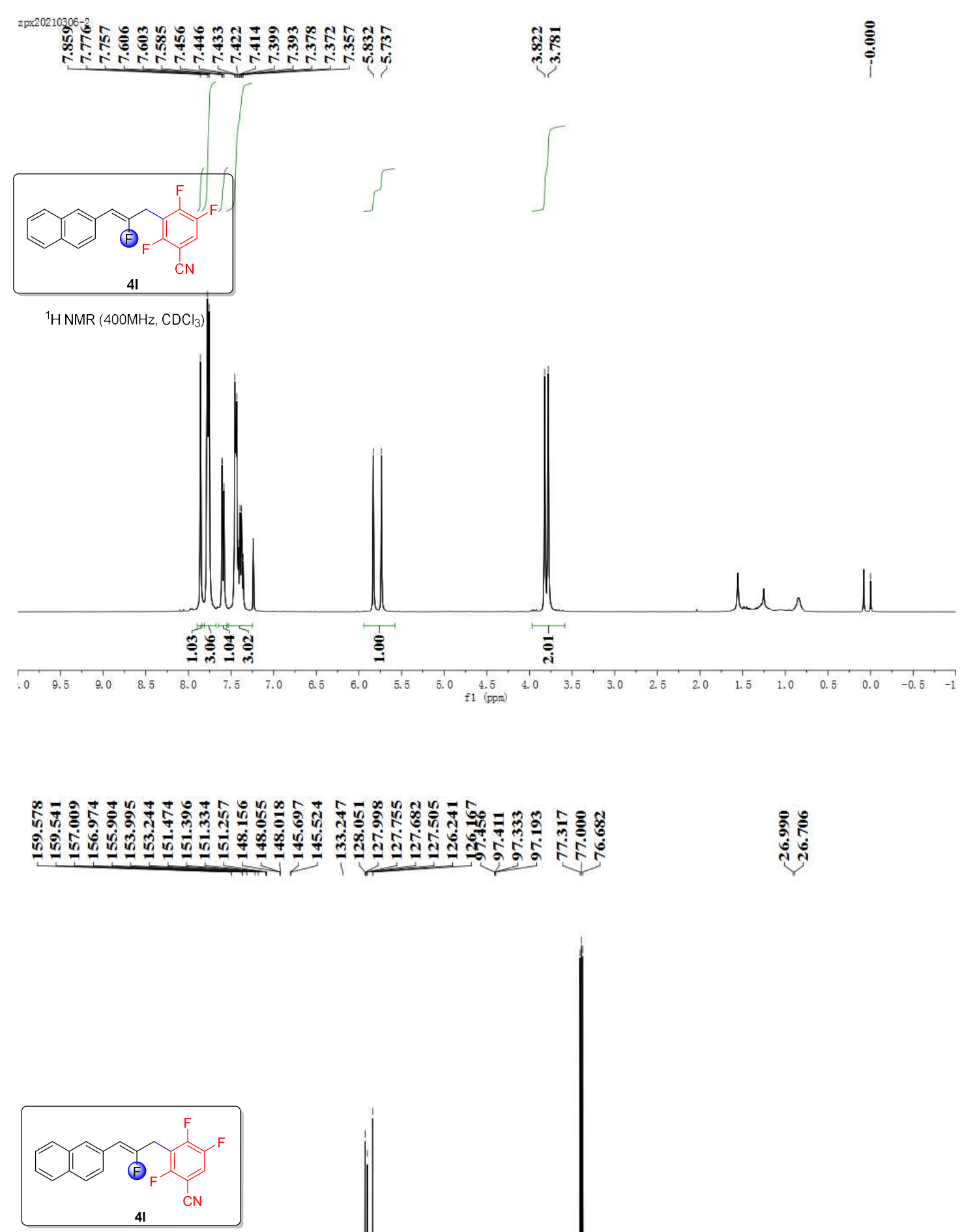

${ }^{13} \mathrm{CNMR}\left(100 \mathrm{MHz}, \mathrm{CDCl}_{3}\right)$

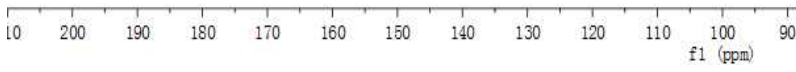



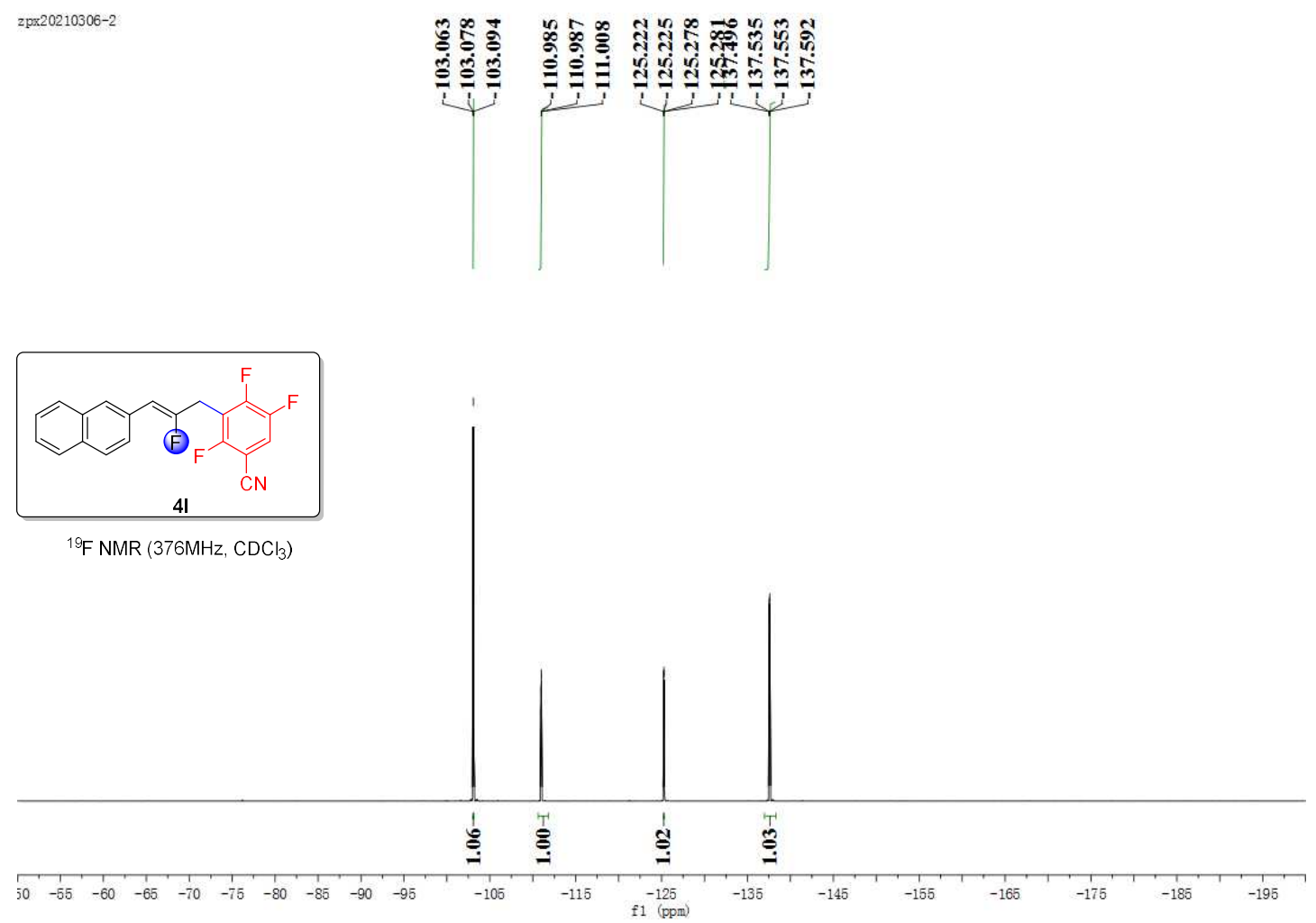